\title{
Select issues in designing license contracts of strategic alliances in the pharmaceutical supply chain
}

\author{
by \\ Amirhossein Mostofi \\ A thesis \\ submitted to the Victoria University of Wellington \\ in partial fulfillment of the requirements for the degree of \\ Doctor of Philosophy
}

Victoria University of Wellington

2022 



\section{Abstract}

Pharmaceutical products play an important role in the human life and improvement of patients. There are many types of medicine for which the supply chain networks have been spread around the world. This extensive application and communication in the logistic of pharmaceutical products entail efficient management and coordination of involved partners. Also, many organizations including private and public organizations as well as financial and insurance companies implement the functions of pharmaceutical services in health systems. Effective management of the partners' relationships in a coordinated and integrated manner requires cooperative strategies. Furthermore, the evaluation of these strategies requires consideration of different aspects of cooperation, participatory capabilities of partners, and necessary incentives facilitating their participation. In this thesis, the establishment of a strategic alliance, between producers of the new and patented pharmaceutical products by the cooperation of main producers or licensor of medicines by local manufacturers, distributors, pharmacies as well as local government are studied. In this regard, pricing of the patented medicines and valuing the license contract are taken into account by regarding different settings and assumptions surrounded the pharmaceutical supply chain. The problems under consideration can be specifically categorized as profit distribution problems in strategic alliances, especially when the alliance is formed with the participation of international partners. As described later, pricing decisions and mechanisms play a crucial role in identifying cooperative profits. Hence, after providing a deep analysis of the pricing mechanisms of the different pharmaceutical products, the cooperative game theory is utilized to address the profit split decisions of the partners under consideration. In doing so, it is attempted to distribute the profits fairly to partners and provide incentives for the participation of them to so-called coordinate them.

The thesis is organized into four sections. The first section discusses a general license contract in the context of an international joint venture where the pricing mechanism of pharmaceutical products is similar to many other products and no special control by the regulatory bodies limits it. In this context, the medicinal raw materials and some medicinal compounds that are supplied in bulk could be mentioned. The results of this section provide managerial insights that could be extended to other special structures of pharmaceutical products and facilitate the extraction of solutions in more complex problems. 
The complex model of an alliance firm considering the financing issue, the demand and market uncertainty, the competitive pricing mechanism in an oligopoly market, the tax and depreciation issues as well as the possibility of the alliance liquidation is studied in the second section. Also, based on the results of the general model of the license contract in the first section, an innovative algorithm based on dynamic programming is proposed to extract the solution of this multi-period stochastic model.

In the third section, the patented medicines are investigated through the establishment of a local manufacturer under a license contract from the main manufacturer. For the patented medicines, also known as branded medicines, the reference-based pricing mechanism is explored. The mechanism is nowadays the most applicable mechanism in many countries as well as the country under study. However, to account for the capabilities of the partners in the cooperative alliance consisting of the main local and main manufacturers as well as the government who affords most of the pharmaceutical-related cost of the patients through franchises themes, the cooperative game theory approach is used to modifies the price of medicines produced now domestically. We regard this approach as an innovative pharmaceutical pricing agreement that plays the role of coordinator of partners by ensuring proper distribution of cooperative surplus to them.

The final section focuses on the deterioration of pharmaceutical products and the effects that a cooperative alliance similar to the one introduced in the third section has on the reduction of this phenomenon. Indeed, we introduce the domestic production of patented medicines as an efficient strategy that could significantly reduce the deterioration of medicines as one of the most worrying challenges of the pharmaceutical supply chain.

For all models discussed in the sections of the thesis, first, the surrounded assumptions of the problem are clarified deeply, and after developing the mathematical models of the problems, the ways for obtaining optimal solutions are explained in detail. Also, the results of the proposed models are compared with the evidence in real-world problems for validation purposes. The thesis ends with managerial insights, conclusions, and future works opportunities in the final section. 


\section{Acknowledgments}

First of all, I am deeply grateful to Allah.

Coming from the intense gratifications of my supervisors whom their contributions

has raised the quality of this thesis.

They have supported me and have given me enthusiasm for research.

They have

patiently guided me. I am very grateful to their supervision and I owe them the greatest degree of appreciation.

I am deeply grateful and I wish to express my deepest gratitude to

\section{A/Prof. Dr. Vipul Jain}

My primary supervisor, Associate Professor of Supply chain Management at Victoria University of Wellington for suggesting the point of the thesis, supervision of the work and for the invaluable guidance, the longtime and tremendous effort to offer every possible help to finish this thesis.

It was a great honor of mine to study under his supervision.

I am genuinely appreciative of $\mathbf{D r}$. Yi Mei for his assistance and undoubted 
effort during his supervision of this work.

\section{$\&$}

My lovely parents, who supported me with love and understanding. Without you, I could never have reached this level of success.

Your prayer for me was what sustained me this far. 


\section{Table of contents}

\section{Contents}

Abstract.

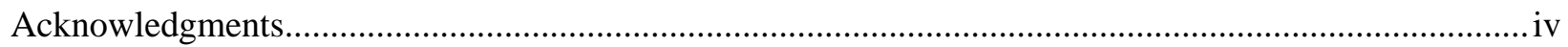

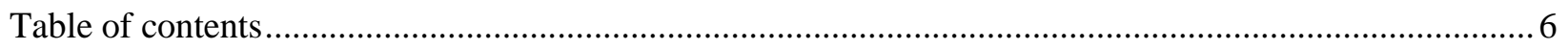

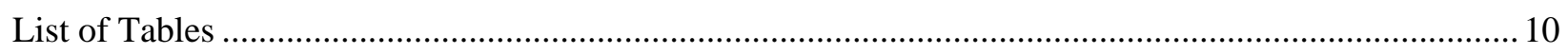

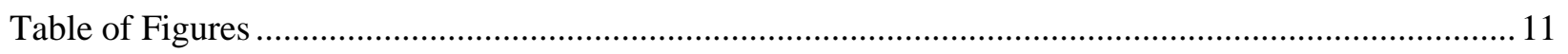

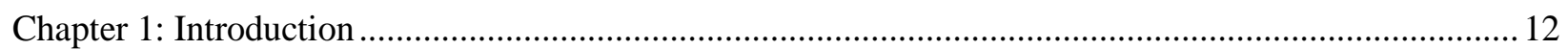

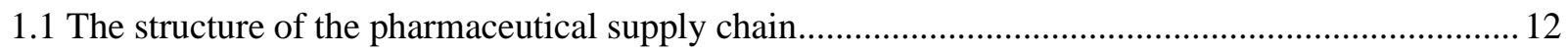

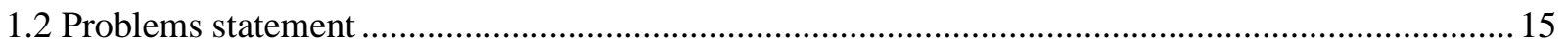

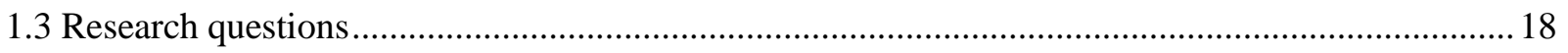

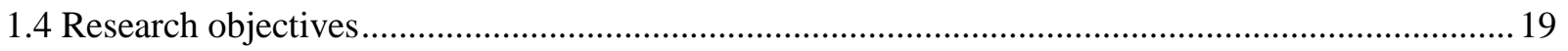

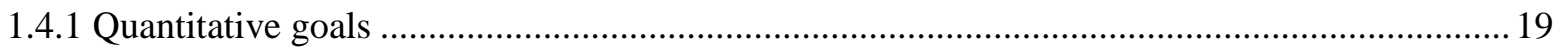

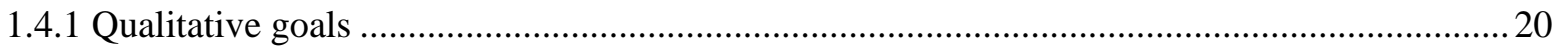

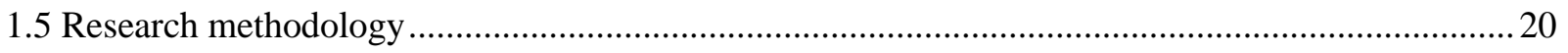

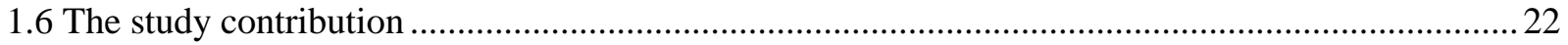

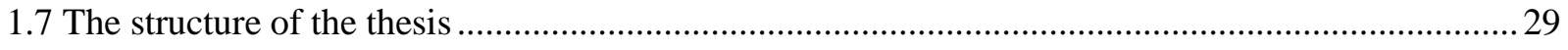

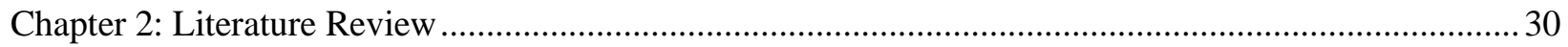

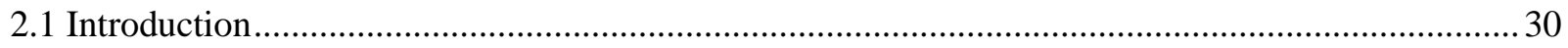

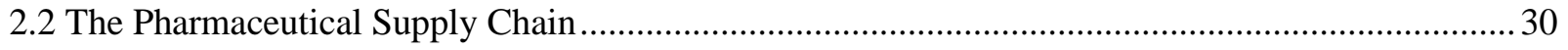

2.3 Product deterioration in the pharmaceutical supply chain .......................................................... 33

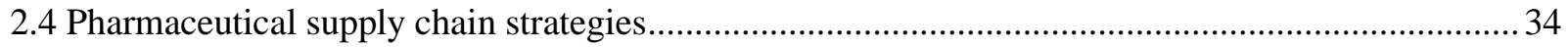

2.5 The strategic alliance and intellectual property valuation........................................................... 35

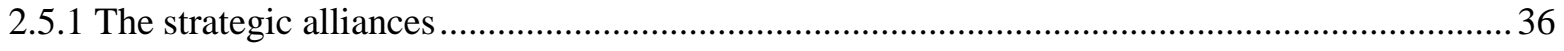

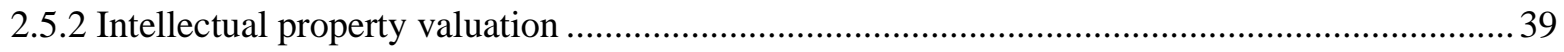

2.5.3 The pricing mechanism of pharmaceutical products............................................................... 40

2.6 Cooperative game theory and the coordination mechanisms .................................................... 43

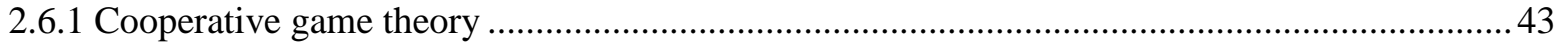

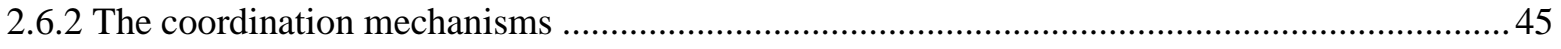

2.7 Learnings from the literature

Chapter 3: Pharmaceutical Alliance: Pricing and Profit Allocation Considering technology and Market

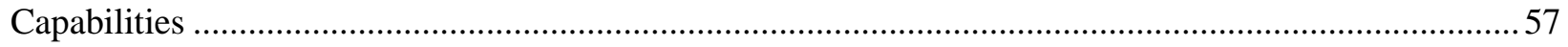

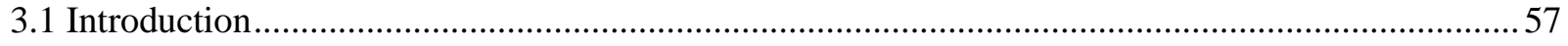

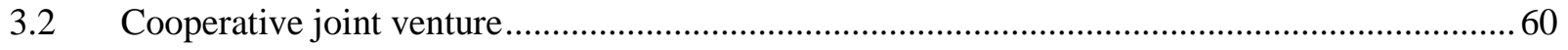




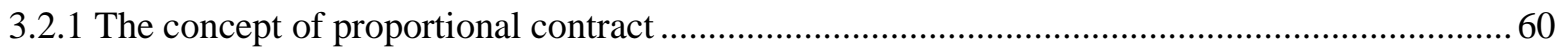

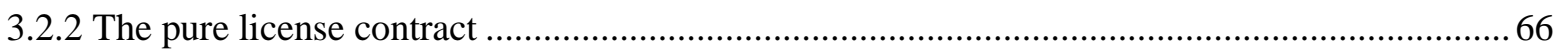

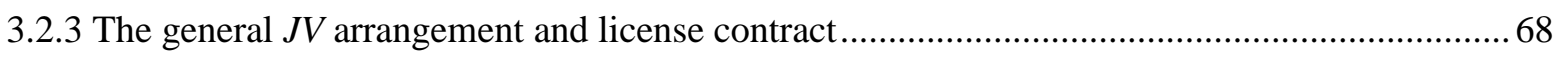

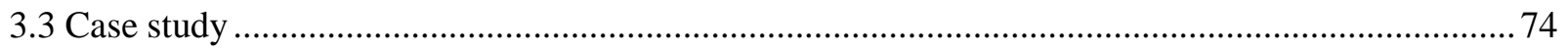

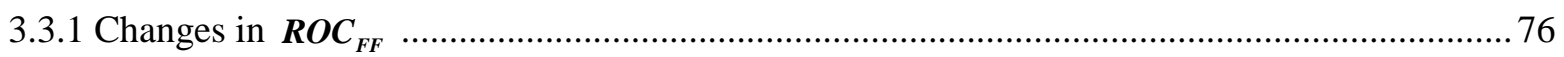

3.3.2 Changes in the market scale parameter $(a)$ and the price-sensitive coefficient $(\boldsymbol{b}) \ldots \ldots \ldots \ldots \ldots . . . .78$

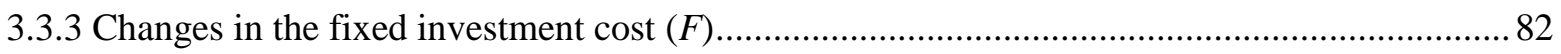

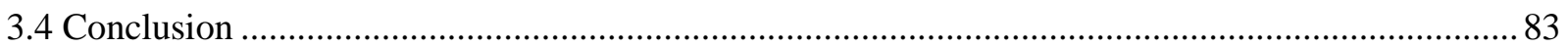

Chapter 4: A new model of the license valuation of pharmaceutical products in an oligopoly market

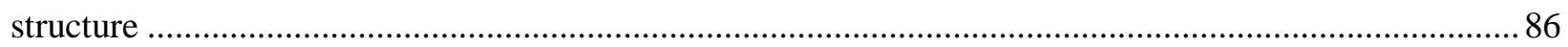

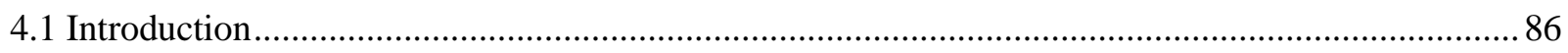

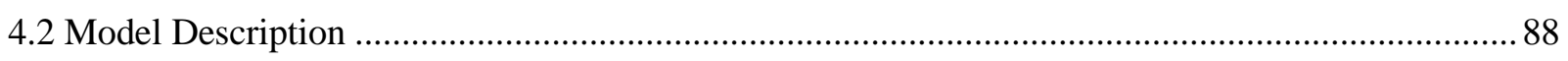

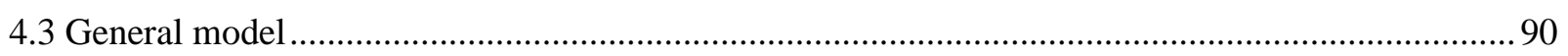

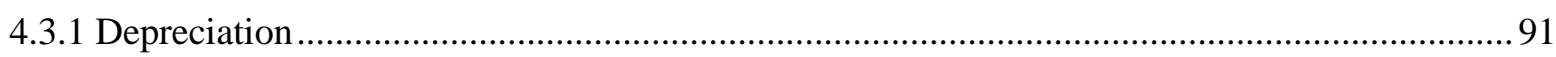

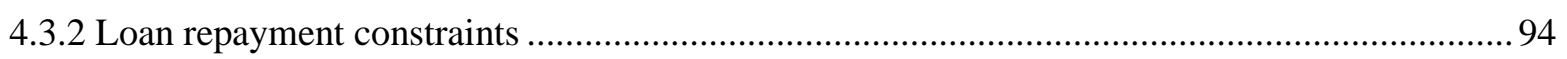

4.3.3 Local market structure considering transaction costs ......................................................... 95

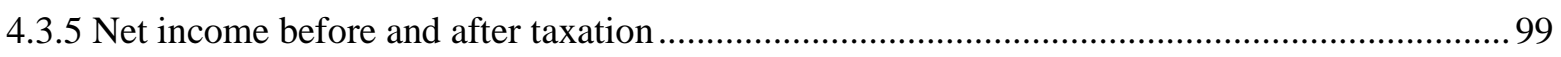

4.3.6 The deterministic model for capacity planning and optimal arrangement of a proportional

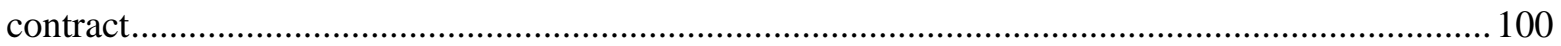

4.3.7 The two-stage stochastic model for capacity planning and optimal arrangement of a

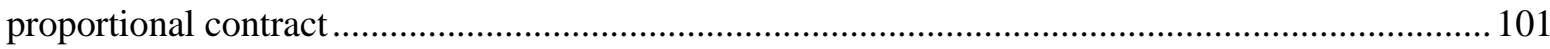

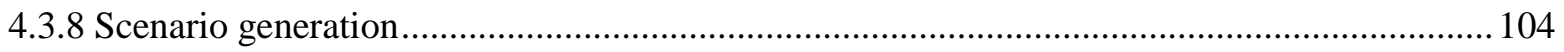

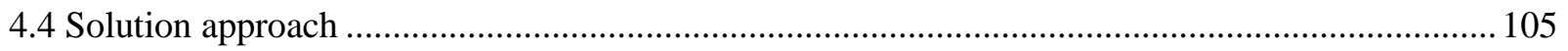

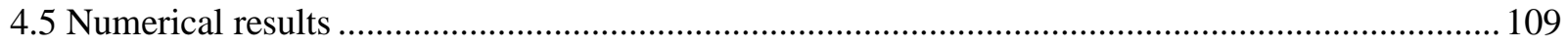

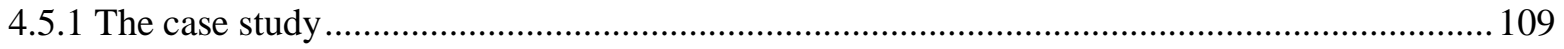

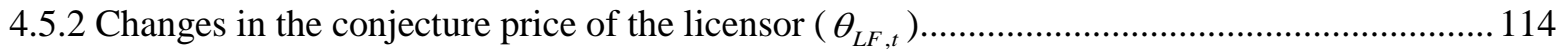

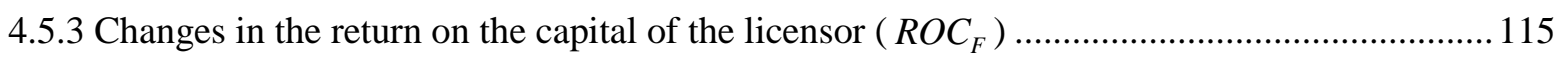

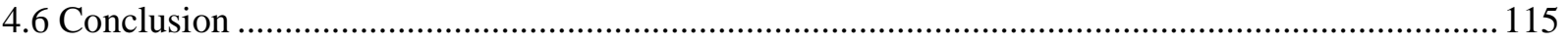

Chapter 5: A new pricing mechanism for pharmaceutical supply chain: A cooperative game theory

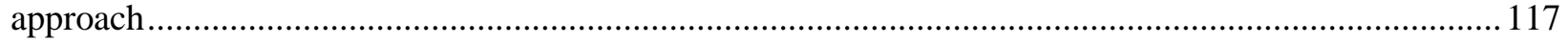

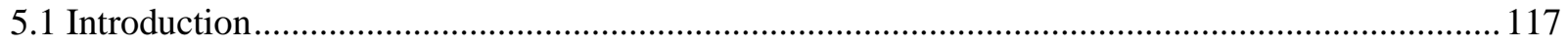

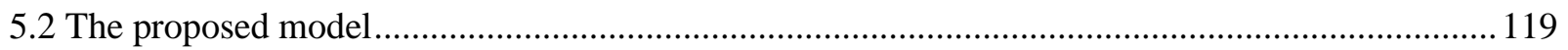

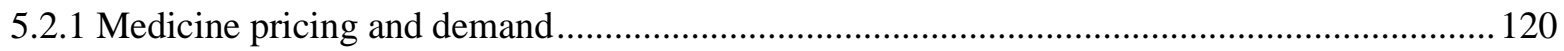

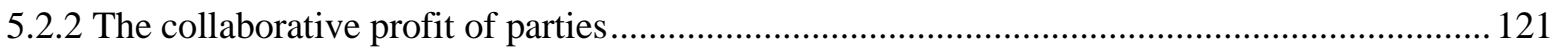

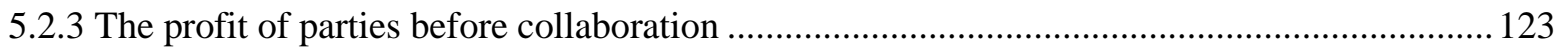




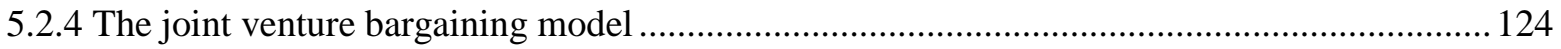

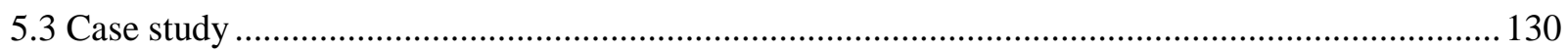

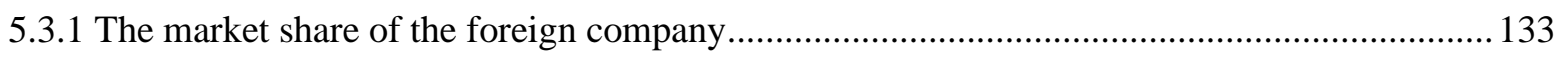

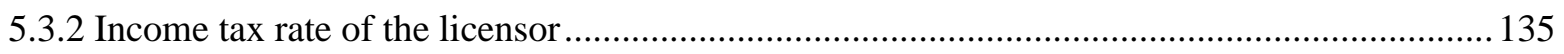

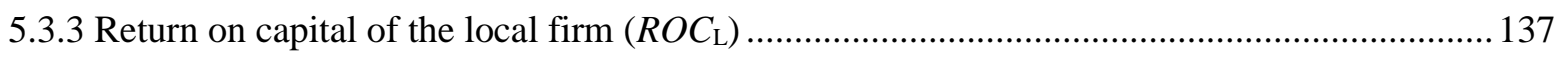

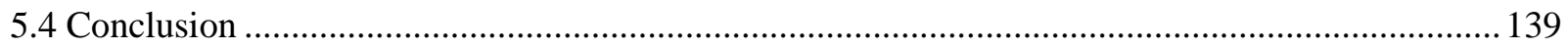

Chapter 6: A new pricing mechanism for licensed pharmaceutical products concerning their deterioration

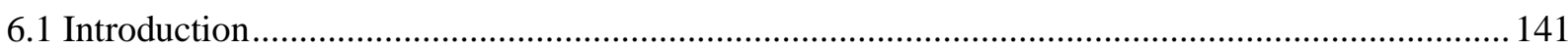

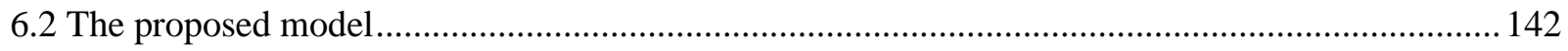

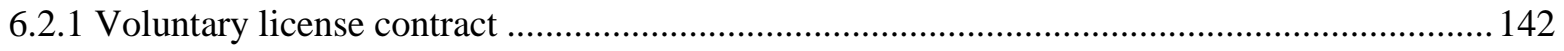

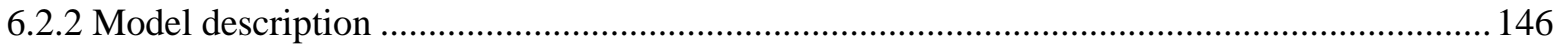

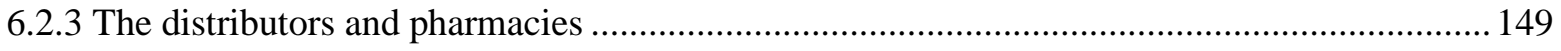

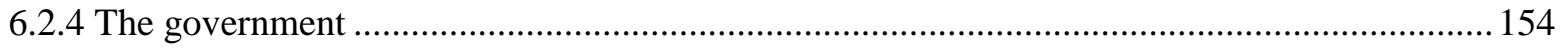

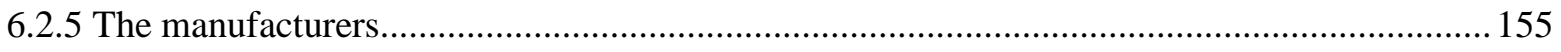

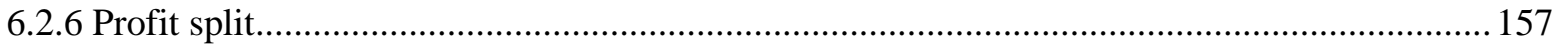

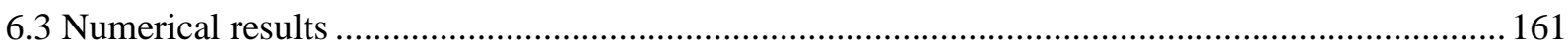

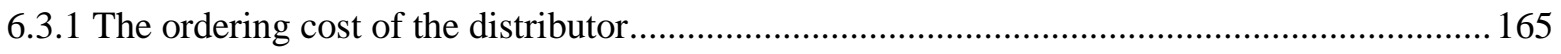

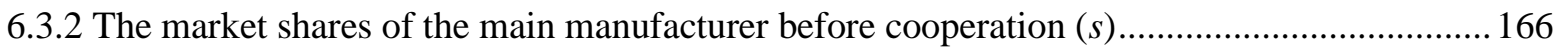

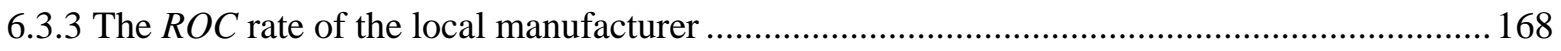

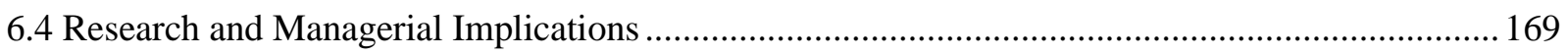

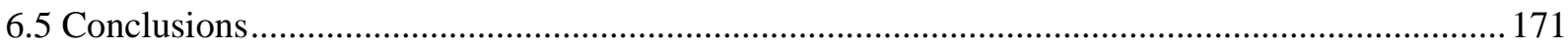

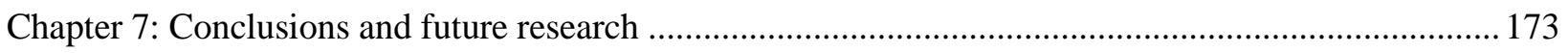

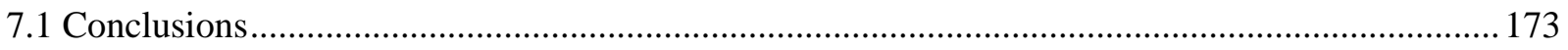

7.2 Significant contribution from current COVID-19 perspective …............................................ 177

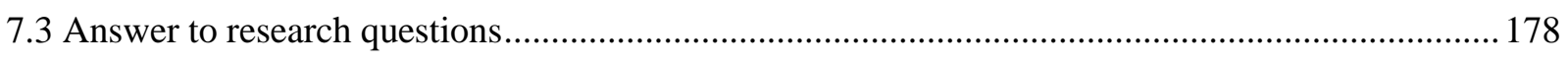

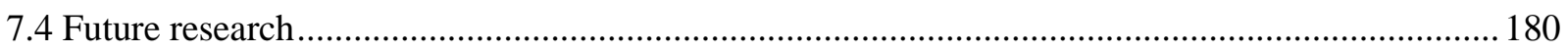

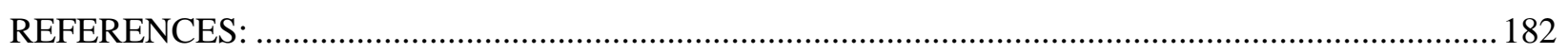

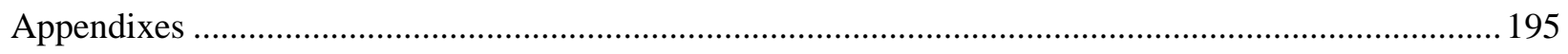

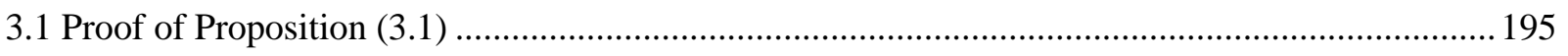

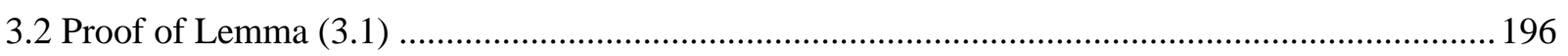

3.3 Proof of the negativity of radicand in Eq. 3.23 when $\left(\boldsymbol{O}_{F F} \cdot\left(1-\boldsymbol{\tau}_{L F}\right)-\boldsymbol{O}_{L F} \cdot\left(1-\boldsymbol{\tau}_{F F .2}\right)\right)>0 \ldots \ldots \ldots \ldots . .196$

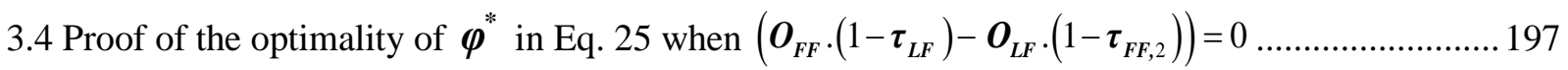


3.5 Proof of the optimality of $\boldsymbol{\varphi}^{*}=0$ in the state where $\left(\boldsymbol{O}_{F F} \cdot\left(1-\boldsymbol{\tau}_{L F}\right)-\boldsymbol{O}_{L F} \cdot\left(1-\tau_{F F, 2}\right)\right)<0$ and $O_{L F} \cdot\left(\tau_{F F, 1}-\tau_{F F, 2}\right)-O_{F F} \cdot\left(2-\tau_{L F}-\tau_{F F, 1}\right) \leq 0$

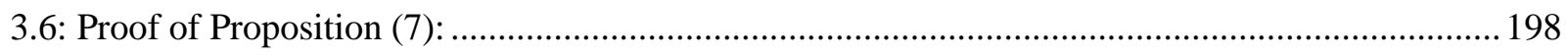

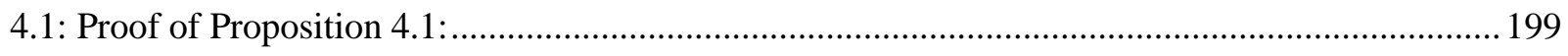




\section{List of Tables}

Table 2.1 The comparison of the first models of thesis by reviewed researches ...................... 51

Table 2.2 The comparison of the second model of thesis by reviewed researches .................... 52

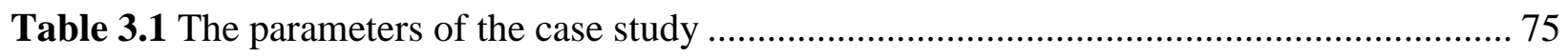

Table 4.1 The amount of supply in the previous years (Kilogram) ................................... 111

Table 4.2 The production cost of supplier in the previous years (\$ / Kilogram) ..................... 111

Table 4.3 The transaction cost of supplier in the previous years (\$ /Kilogram) ...................... 112

Table 4.4 The market price of product in the previous years (\$ /Kilogram).......................... 112

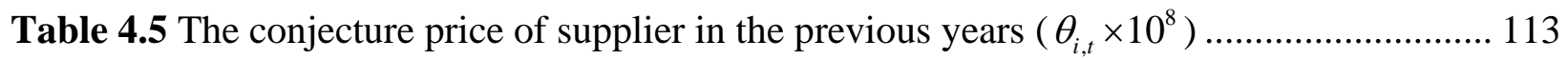

Table 4.6 The results of the proposed model for different level of capacity .......................... 113

Table 5.1 The results of revenue distribution in equal sharing mode .................................. 132

Table 6.1 The value of parameters in the case study ...................................................... 162

Table 6.2 The optimal policy of distributors and pharmacies in the non-cooperative strategy . 163

Table 6.3 The optimal policy of distributors and pharmacies in the cooperative strategy ........ 164

Table 6.4 The results of the model for the considered case study ....................................... 164

Table 6.5 The results of the cooperative model against the non-cooperative ordering cost of distributor 166

Table 6.6 The results of the cooperative model against the market shares of main manufacturer 167

Table 6.7 The results of the cooperative model against the return on the capital rate of the main manufacturer 169 


\section{Table of Figures}

Figure 1.1 A general pharmaceutical network .................................................................. 15

Figure 1.2 The boundary of the supply chain in the previous studies .................................... 16

Figure 1.3 Hierarchical model type one................................................24

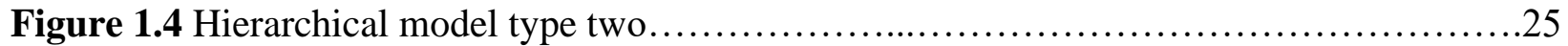

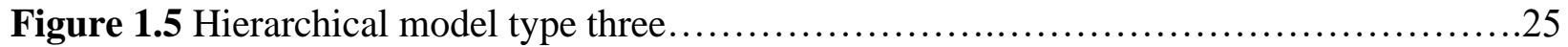

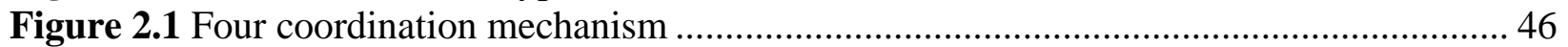

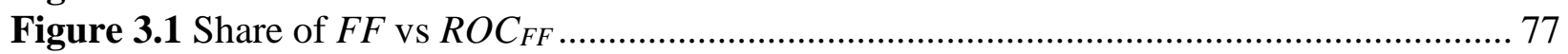

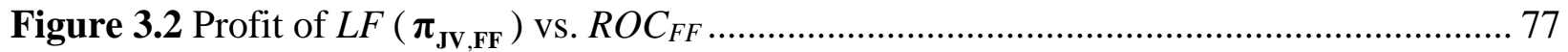

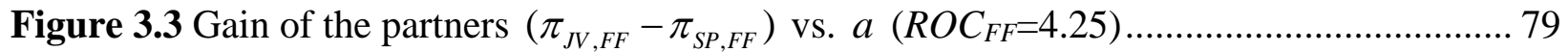

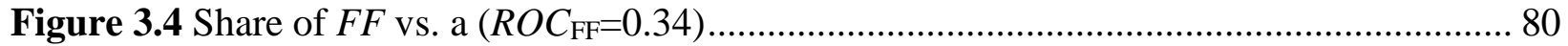

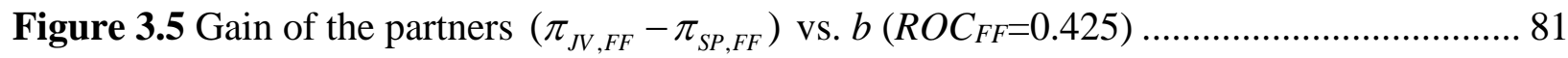

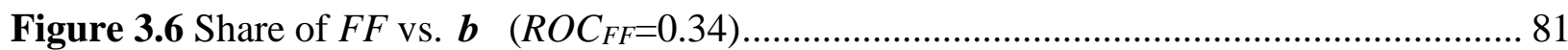

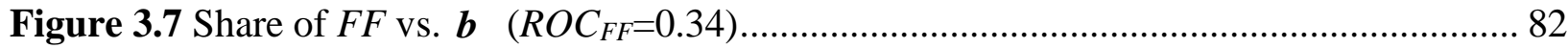

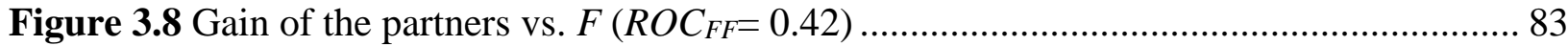

Figure 4.1 Asset value over time in the straight depreciation method.................................. 92

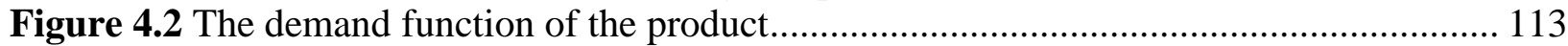

Figure 4.3 The partners' gain versus the conjecture price of the licensor ............................. 114

Figure 4.4 The partners' gain versus the return on the capital of the licensor ........................ 115

Figure 5.1 The position of the optimal solution of the Nash bargaining model between the

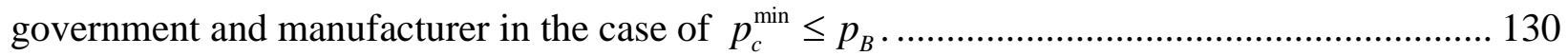

Figure 5.2 $p_{c}^{\mathrm{min}}$ against the market share of the foreign company ....................................... 134

Figure 5.3 Changes in the equilibrium price relative to the market share of the foreign company

Figure 5.4 $p_{c}^{\min }$ 34against income tax rate of the foreign company ..................................... 136

Figure 5.5 Changes in the equilibrium price in relation to income tax of the foreign company 137

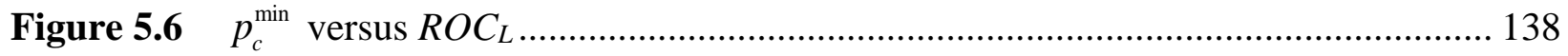

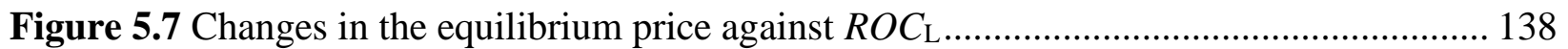

Figure 6.1 Inventory Level of distributors and pharmacies (Wang et al., 2011) ..................... 151

Figure 6.2 The deterioration cost vs. the ordering cost of distributors in the non-cooperative

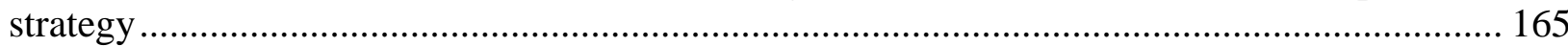

Figure 6.3 The cooperative gain of local manufacturer vs. the market shares of main manufacturer 167

Figure 6.4 The cooperative gain of the local manufacturer vs. the market shares of the main manufacturer 168 


\section{Chapter 1: Introduction}

The pharmaceutical products and services are extensive and account for a massive amount of costs in health care systems. The cooperation of involved partners in providing the required services has remarkable consequences on improving efficiency and reducing costs. The thesis focuses on forming a strategic alliance to produce the patented or brand pharmaceutical products by a license contract whereby the main manufacturer grants the production rights to a local or domestic manufacturer. The evaluation of this strategy, the pricing decisions of the products in the alliance, and the profit distribution of partners are the main subjects investigated in this thesis. The strategy also has effective consequences on the performance of the pharmaceutical logistic network and provides remarkable opportunities for partners involved in the provision of pharmaceutical products, including manufacturers, distributors, pharmacies as well as the government.

In this section, the concepts and definitions of the pharmaceutical supply chain used throughout the thesis are described. Next, various aspects of the problem under study are stated. After the problem statement, the research questions, objectives, and methodology are declared. Finally, the organization of the dissertation is described.

\subsection{The structure of the pharmaceutical supply chain}

A pharmaceutical supply chain is a combination of operations, processes, and the relationships between them to discover, develop and manufacture pharmaceutical products (Shah, 2004).

The World Health Organization (WHO) defines medicine as any substance or mixture of substances that are manufactured for sale and distribution in order to use in:

(i) the treatment, mitigation, cure, prevention, or diagnosis of disease

(ii) the restoration, correction, or modification of organic functions in humans or animals. A pharmaceutical supply chain can be considered as part of the healthcare delivery system (HDC). The WHO defines HDC as a composition of organizations, institutions, resources, and individuals committed to delivering fair and efficient services that are essential for achieving or improving healthcare. 
According to this definition, various key factors are involved in the pharmaceutical supply chain. These include:

$>$ Large and multinational research and development centers that present their products in global markets.

$>$ General manufacturers that produce off-patent and generic medicines.

$>$ Local manufacturers produce generic and brand-name medicines by obtaining the necessary licenses.

> Contract manufacturers either manufacture pharmaceutical raw materials or final products by outsourcing to companies that have the necessary technical knowledge.

$>$ Biotechnology or medicine discovery companies that are knowledge-based start-ups; though these companies have the necessary knowledge, they lack significant production capacities.

The first group dominates the marketplace, and supply chain issues are more relevant to them (Shah, 2004).

The healthcare sector has progressed remarkably in recent years in a way that the mortality rate has fallen by more than $10 \%$ between the years 1960 and 2014. Various factors have contributed to this reduction, including the improvement of medical care, access to the needed medicines, and the availability of high-tech facilities. Supply chain management is critical because it provides the right dosage of medicines at the right time for patients, and it can be effective in patients' recovery (Schaeffer, 2009).

New challenges have been raised in the pharmaceutical supply chain that affect the benefits of this chain Booth (1999). These include:

$>$ Advanced R\&D activities offer new pharmaceutical products that compete with the existing patents.

The effective patent terms are decreasing due to international regulations.

Patents have fewer market limitations due to new regulations.

$>$ Regarding the high costs of medications paid by the patients, new products are approved if they are cost-effective and yield significant benefits. 
Moreover, in some studies, such as Booth (1999), the goal of the supply chain is to create value for the customers in addition to the profitability of partners. Hence, establishing domestic manufacturers that reduce the price of products, pharmacy inventory management, and the reduction of costs such as transportation and deteriorated products are the objectives of this thesis in line with the social welfare concepts of the supply chain.

Several studies have also been conducted on estimating the lifespan of pharmaceutical products. According to Shah (2004), the test of medicine effectiveness in R\&D centers (10 years), obtaining patent protection, safety and efficacy test (6-8 years), and finally distribution to the market formed the stages required to introduce new medicines to market. This tremendous amount of effort and the short shelf life of patents has encouraged manufacturing companies to take cooperative strategies to reduce costs and maximize market profitability.

Therefore, the advantages of the developed cooperative alliance-based models in this study are to reduce costs and increase profitability and market share is in line with real-world trends, thus, improving the performance of the pharmaceutical supply chain. This arrangement arises in the design stage of a pharmaceutical supply chain and plays a vital role in estimating the development costs as a new firm's valuation model involved the pharmaceutical supply chain will result in a proper and smooth flow of information across various players involved in the chain.

The generic considered pharmaceutical supply chain in this study includes the following components: primary manufacturer, secondary manufacturer, distribution centers, and retail outlets, including pharmacies and hospitals. Figure 1.1 illustrates a general pharmaceutical network. The primary manufacturers produce the active ingredients, which are the chemical and molecular components of the medicine. Each stage of the production in such manufacturers such as quality control, changeover, and cleaning takes a long time which is the intrinsic characteristic of production in primary manufacturers. Secondary manufacturers mix excipients and active gradient composition and then produce, package, and control the quality of the medicines. Distribution companies usually have a significant role in marketing products by obtaining the necessary permissions. These companies are an integral part of the supply chain. Moreover, based on the purposes of a $\mathrm{PSM}^{1}$, other players such as medicine inspection laboratories (Imran et al., 2018), Managed Care Organization (MCO), Healthcare Maintenance Organization (HMO) (Shah,

\footnotetext{
${ }^{1}$ Pharmaceutical Supply Management
} 
2004), and other governmental or administrative bodies could be included in PSM, and usually, they concern with the quality issues or quality control process.

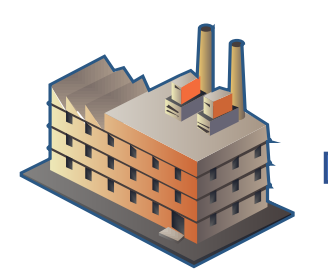

Primary manufacturer

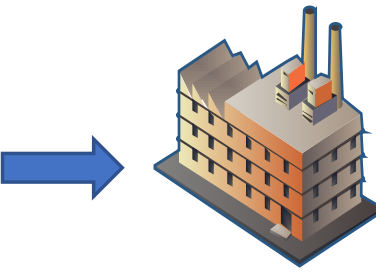

Secondary manufacturer

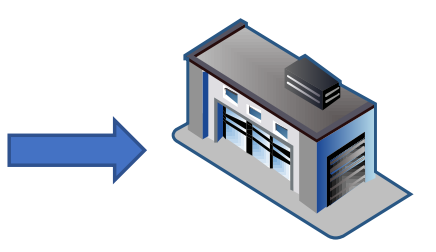

Distributor

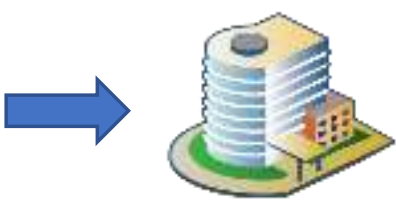

Retail outlet

Figure 1.1 A general pharmaceutical network

\subsection{Problems statement}

The considered network under study shown in Figure 1.2 consists of one or several technologybased pharmaceutical manufacturers, one or more distributors, and demand points, including hospitals and pharmacies. In this network, before cooperation, the main distributor imports the considered medicines from a secondary manufacturer which is in another country than that of the distributor or the local market. Then, the distributor transports the medicines to the demand points appropriately. However, after cooperation, a local producer is formed by the cooperation of the secondary manufacturer and the main distributor in the local country. Accordingly, the raw material of the medicines is imported from the primary manufacturer to the local secondary manufacturer in the latter case. The effects of such arrangement are on-time production, reduction in ordering cost, transaction cost (due to the conveniences provided by the local government), and deterioration. 


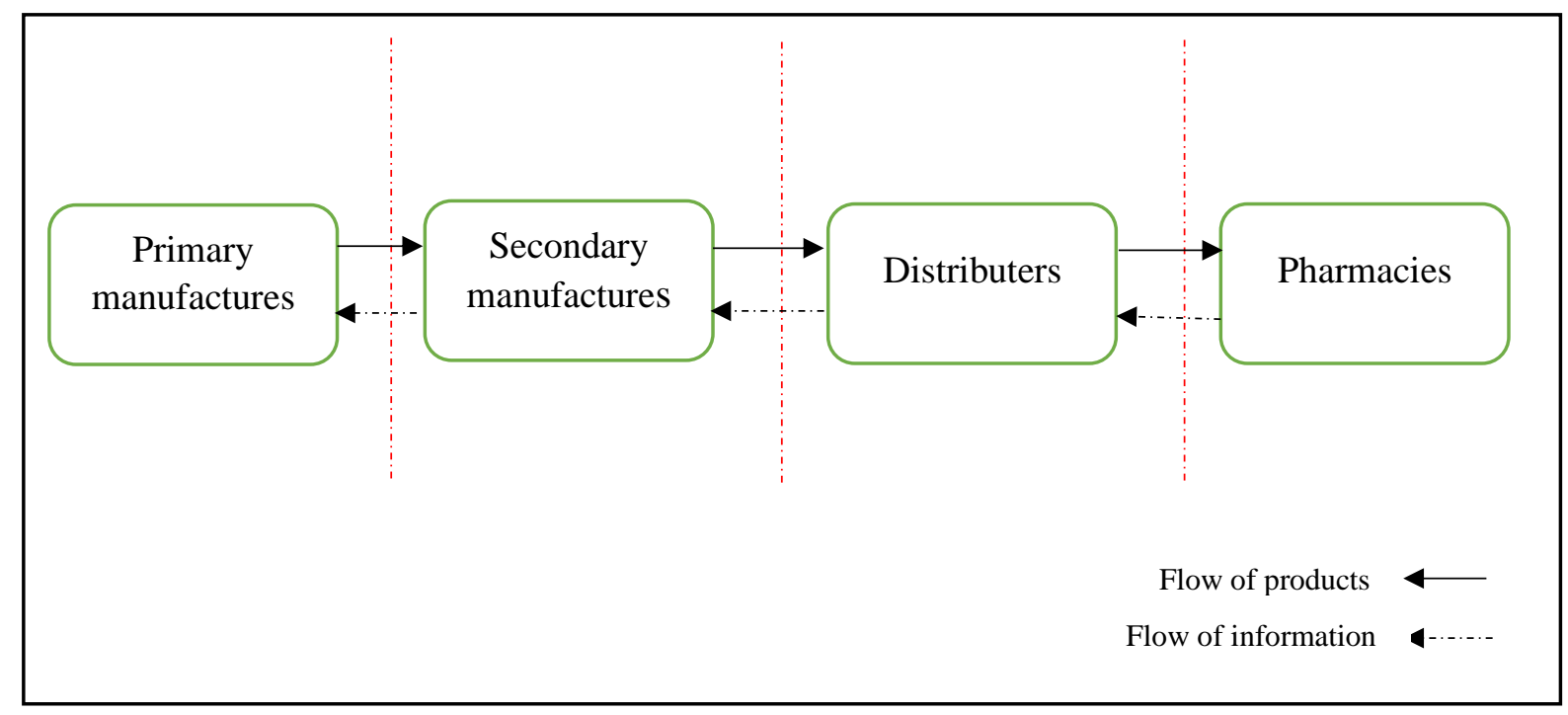

Figure 1.2 The boundary of the supply chain in the previous studies

The study's main objective is to determine how the cooperative alliance is evaluated, especially in the context of the pharmaceutical industry. Notably, an asset has no worth by itself, and its value is tied to the ongoing outcomes or utility that the asset will bring for its owner. This paradigm forms the main idea of the valuation models, and such models are worthy when the intellectual properties are some part of the asset. In our study, the medicines' formulas and the required equipment to produce the medicines are the rare resources that are owned by the producers, whereby they expect high value for such prosperities. So, evaluating supply chain partners' capabilities is key to determine the value of the alliance and the share of partners of the cooperation profits. We investigate a mathematical modeling approach for addressing the valuation process as well as the alliance decisions. Hence, incorporating the valuation process of firms with the modeling approach to support strategic decisions is the main contribution of the thesis.

The most important factors that affect the valuation of the alliance and its modeling include the partners involved and how they interact, market structure and pricing mechanisms of pharmaceutical products, partners' capability to create synergies and reduce costs, and the level of detail that the model addresses. Based on these factors, four models are proposed to address the valuation problem in PSC and how the profit of the partnership is split among the partners. These models are presented in 4 separate sections, and in the following, a brief description of them and the challenges they study is discussed. 
In the first model of the thesis, the general license contract of international alliances by focusing on the capabilities of the partners and the effect the capabilities have on the ownership structure of the alliances is investigated. The model discusses whether the shared ownership of partners is optimal or exposing the brand medicines through royalty payments paid by the local manufacturer to the main manufacturer provides better outcomes for the alliances. In this regard, as shown later, the tax rates on the incomes of the partners and their ability to convert invested costs into profit in their own business are key factors affecting the optimal structure of the ownership.

In the second model, the cooperative alliance is valued by considering financial considerations and in line with financial research. This model also includes decisions related to ownership structure, capacity planning, alliance financing, equilibrium pricing, future alliance dissolution, and the contract parameters between main and local manufacturers. According to the valuation method, the value of the alliance is related to its ongoing profit and is subject to uncertainty in the model parameters in the future. Therefore, the model will be a multi-period mathematical programming model with uncertainty consideration using stochastic programming. Also, similar to the option valuation methods, the possibility for dissolution is embodied in the model if the book value of assets at the time of dissolution is estimated to be greater than the present value of the future outcomes. Clearly, the future outcomes depend on future market sales. Hence, to reflect the market situations, various scenarios are presented in which even the possibility of shock in the market for reasons such as the introduction of new medicines is demanded.

The interactions between the government and manufacturers are explored in the third model of the thesis. Usually, the governments support local and main manufacturers' alliances by granting the exclusive production right to them. However, due to the extremely expensive government costs in the health systems and their role in granting the exclusive production right, they expect some discount on the price of supplied medicines. Therefore, by evaluating the total saving of the alliance on the pharmaceutical-related costs, a negotiation process based on the Nash bargaining solution of the cooperative game theory is utilized to determine the profit of PSC partners as well as the saving on the costs of the government.

As a fundamental challenge of the pharmaceutical supply chain, the deterioration of products is taken into account in the final model. In this model, the high ordering costs of imported brand medicines are regarded as one of the reasons for the high deterioration of products. On this point, 
the establishment of the cooperative alliance enables local and just-in-time production of medicines, consequently reducing the order quantity orders of distributors and pharmacies and reducing the deterioration-related costs. By taking into mind this advantage of the alliance, we explain how the profits of the cooperation could be split between partners using the cooperative game theory again. It is also illustrated that this strategy could diminish the deterioration of medicines and prepare advantageous beyond the economic considerations.

It is also notable that all proposed models are presented in decentralized and centralized modes. Decentralized models refer to the best responses of the partners before the cooperation while centralized models investigate the best outcomes for the supply chain as a whole and in an integrated style. Moreover, the license contract in this thesis is regarded as a coordinated contract that is regulated in such a way that properly distributing the cooperative profit to the partners, provides the necessary incentives for them to cooperate. Like many coordination studies, the cooperative game theory and the Nash bargaining solution are utilized to obtain such a contract.

\subsection{Research questions}

The goal of this Ph.D. research study is to design select issues in a pharmaceutical supply chain and further develop decision-making models to enhance the performance of the considered pharmaceutical supply chain. The proposed models will provide the supply chain practitioners and decision-makers an efficient and effective framework with more cooperation between players by cooperative alliance networks, resulting in a smooth and proper flow of timely information to the players involved in the considered pharmaceutical supply chain.

More specifically, this study seeks to provide answers to the following research questions:

1. How can the new design of the supply chain with a smooth and proper strategy increase the pharmaceutical supply chain's performance? Does this new design provide the customer with the same medicine at less price and less time, resulting in better customer satisfaction?

2. How can we measure a cooperative alliance's value and determine the share of profits for the various partners involved in the intended alliance? Does the proposed cooperative alliance provide a "win-win" situation for the players? 
3. How can we control and reduce pharmaceutical products' deterioration rate and how beneficial are these developed models for customers?

These research questions have led to the development of research objectives in the next section.

\subsection{Research objectives}

We separate the quantitative and qualitative goals of the research as the following:

\subsubsection{Quantitative goals}

1) Valuation of a cooperative alliance by considering the contribution of each partner in providing Intellectual Property (IP), network information and required the permission of the local government to enhance the performance of PSC.

2) To propose methods for controlling and reducing medicine deterioration regarding the high damage caused by deterioration in PSC (the policies of distributor-managed inventory and distributor-secondary manufacturer integration through a license contract).

3) To propose a research framework for predicting the price of pharmaceutical products, considering uncertainty in future periods as well as the market structure.

4) To measure the incurred cost of producing, holding, and delivering the medicines.

It is notable that to validate the strategic alliance, the value-added activities which cooperatively reduce the cost and increase the profitability should be considered. Therefore, granting the exclusive market to the alliance and its effects on reducing the deterioration (Quantitative goal 1) are amongst the value-adding activities used in the valuation method (Quantitative goal 2). Also, in the valuation methods based on the resulting revenues, the forecasting of demand has significant roles for valuation purposes and pricing decisions. So, quantitative goal 3 states that if the uncertainty is estimated satisfactory and according to the valid methods, then the $I P$ valuation method will be acceptable and confident. So, to reach quantitative goals 1 and 2, the assumed market structure (quantitative goal 3) should be scrutinized intensely. Finally, when we optimize the costs of the supply chain, this cost could be scratched to the total produced medicines and provides a good estimation for the cost of products that further is adjusted with the mark-up to determine the medicines' acceptable price by the legal agencies. 


\subsubsection{Qualitative goals}

The qualitative objectives of this study are:

1) We are presenting an initiative strategy in the pharmaceutical supply chain, which increases the social welfare and sustainability aspects besides the supply chain's efficiency. In this regard, it should be noted that the goals of health care systems are beyond the partners' selfinterest and are committed to protecting human beings as well as possible. In this regard, in most health systems, free pricing is not allowed, and the social agencies control the price by considering some allowable mark-ups for supplying health services. Considering a cooperative strategy, which is also encouraged by the governmental administrative, contributes to social welfare by providing cheaper medicines and, faster, for patients. This strategy improves the agility of the supply chain and decreases the cost of medicines by reducing the cost of providing such medicines. Also, this strategy increases the market share of the considered medicines, and if the strategy accompanies the legal protection, then sometimes importing of similar medicines is forbidden in the local countries (as done in Iran). This qualitative goal is in line with quantitative goal 4. Also, when we reduce the deterioration of medicines, the expired medicines are decreased, which has a positive impact on other sustainable aspects such as water pollution used in disposing of the deterioration of medicines.

2) Improving supply chain performance through managerial and technical decisions: in the present study, reducing pharmaceutical products' deterioration rate using these policies will improve supply chain performance (quantitative goal 1). Moreover, investment in pharmaceutical technology with the participation of a technology providing firm (quantitative goal 2) not only enhances the ability of the supply chain to respond to the requirements of the customer as soon as possible (Agility) but also increases supply chain efficiency (lean production) by reducing the costs.

\subsection{Research methodology}

This thesis's methodology is mainly based on a mathematical modeling approach and using optimization techniques to extract the solutions as the policies of the proposed cooperative strategy. In the modeling approach, first, the surrounded assumptions of the problems are clarified. 
These assumptions are derived from standard approaches to real-world problems adjusted according to the problems studied. The general terms of the license contract are stemmed from the international joint venture contract (Sercu, 2009), and their parameters are adjusted according to the problem and the pharmaceutical supply chain environment. To take into account the partners' capabilities in providing the distribution network, technological knowledge, and the market share and structure, appropriate mathematical models are proposed by modifying the existing models in the literature.

In the present study, I will model the supply chain of pharmaceutical products in both cases of independent decision-making and integration with a secondary manufacturer of pharmaceutical products. In the independent mode, the current decision-making environment of partners is considered to identify the outcomes of them in the lack of cooperation. In this case, as stated later, the products import from main manufacturers and distribute to patients through the network of local partners. On the other hand, the cooperative case is addressed when medicines are produced internally with the participation or permission of a secondary manufacturer. In this case, due to the low ordering costs (no need for formalities like customs, etc.), customers can control and manage the inventory of pharmaceutical products while the distributors, in cooperation with the secondary manufacturer, undertake to provide their customers with freshly-made products. The proposed models in this thesis will be according to the assumptions above. We could compare the cost benefits of both cases of the supply chain and examine which of them is better. So, modeling and solving two cases provide us with insights into the validity of the proposed models. Accordingly, in line with the concept of system theory, we expect that the cooperative model improves all partners' performance as a whole and we will discuss through a license contract how this contract could provide intensives for the integration of the partners as a coordination mechanism.

On the other hand, I aim to evaluate the value of the pharmaceutical supply chain's intellectual properties by combining the game theory and the valuation methods concept. The Longstaff and Schwartz (2001) model can be introduced as one of the most efficient valuation models. In the present study, I will use the concepts used in this model as the basis for valuation in the fourth model of theses, i.e., we will calculate the future net incomes of the cooperative firms (distributors and the secondary manufacturer) and discounting them to time zero in order to compute the net present value of the assumed firms as the value of its activities. Also, according to valuation 
methods, we will apply uncertainty in the process of valuation through mathematical models and consider the possibility of liquidating the company as an option in the model. Therefore, the partners' cooperative model will be based on the valuation methods concepts covered by a mathematical programming model as one of the thesis novelties. For example, to consider the possibility of liquidation, we will define a binary variable with the value of 1 if it is decided based on the model that the continuation of the alliance in the current period is still optimal. However, the binary variables will take the value of zero in the current period and the later periods if the liquidation of the alliance is decided for reasons such as the low estimated present value of the future incomes. Therefore, the research model will be by the valuation research, and thus, will be valid. Finally, using cooperative game theory and the guidelines presented in (Sercu, 2009), we can calculate the license contract parameters through the proposed models. (Considering the synergy resulting from cooperation).

Meanwhile, the resulting parameters could be compared with the value of them in the real-world instants to observe that there is a significant difference between them or not. For example, if the royalty fee for the desired product is $30 \%$ of the total sales by solving the cooperative model, then we can compare the royalty fee of this product with the existing standards such as standardpoors.com ${ }^{1}$. Therefore, if the differences between the two rates will be negligible, we could say that the results of the models are valid.

\subsection{The study contribution}

This thesis contributes to both theoretical and practical aspects. The contribution of these aspects has been presented in the following section.

1. Integrating knowledge domains: In this thesis, a wide range of literature from related domains will be reviewed. The proposed taxonomy is pharmaceutical supply chain management.

2. Contribution to policy-making: This thesis will contribute to management policies to reduce the deterioration of pharmaceutical products. The research models presented in the study provides:

\footnotetext{
${ }^{1}$ https://www.spratings.com/documents/20184/86990/Medicine_Royalty_II_6_17_14/4a028c54-f12a-4f75-a936-935172f86021
} 
A valuable management perspective in reducing product deterioration

Methods for decision-makers in this area to adopt critical inventory control policies.

Inventory control policies with considering uncertainty in demand as well as existing costs.

Clarify the role of partners in preparing the surplus and synergy of the cooperative alliance.

\section{Contribution to method development:}

Using the unconstrained non-linear programming method and first and second-order conditions to determine the optimal policies of partners in pricing decisions as well as the policy of inventory system of distributors and pharmacies.

$>$ Using mathematical programming to estimate the cost components of the cooperative alliance $(J V)$.

$>$ Providing a research model for managers to estimate uncertainty in PSC through stochastic programming.

Proposing an innovative dynamic programming method to determine the optimal solution of the proposed stochastic programming model for valuing the alliance.

4. Contribution to theory building: This study revisited the theories of network design and cooperative alliances in pharmaceutical supply chain management.

$>$ In a reviewed paper on joint investment alliances by Hong and Chan (2014), they introduced the lack of mathematical models that study different titles in these investments as the biggest challenge in academic research in this field. It has also been pointed out that most research studies are qualitative, which merely investigate the factors of success or failure of these alliances through descriptive research considering experts' opinions.

This study which specifically addresses the issue of valuing the pharmaceutical alliances, distributing revenues, or allocating profits to partners, and measuring the 
benefits of partners, is one of the few studies that fill the existing gap of research in this area.

Computing the stand-alone profit of the partners using the decentralized models.

Providing a structured framework for evaluating the IP assets as well as the network information and required permission owned by the local partner in a PSC.

This is thesis implements a hierarchical planning approach with various modeling structures. There are three types of hierarchical planning which are considered in this research. The first type is about different levels of decision making that the output of a level is used as the input of another level. Therefore, the second type could be non-feasible. In fact, the second level model defines some constraints as a feedback mechanism. Figure 1.3 illustrates the first type of hierarchical planning. Wu and Ierapetriou (2007) modeled production and scheduling operations with hierarchical planning, identifying the scheduling of the tactical horizon of production planning in the first level. Then, this production plan is considered the input of scheduling and production planning at the operational level.

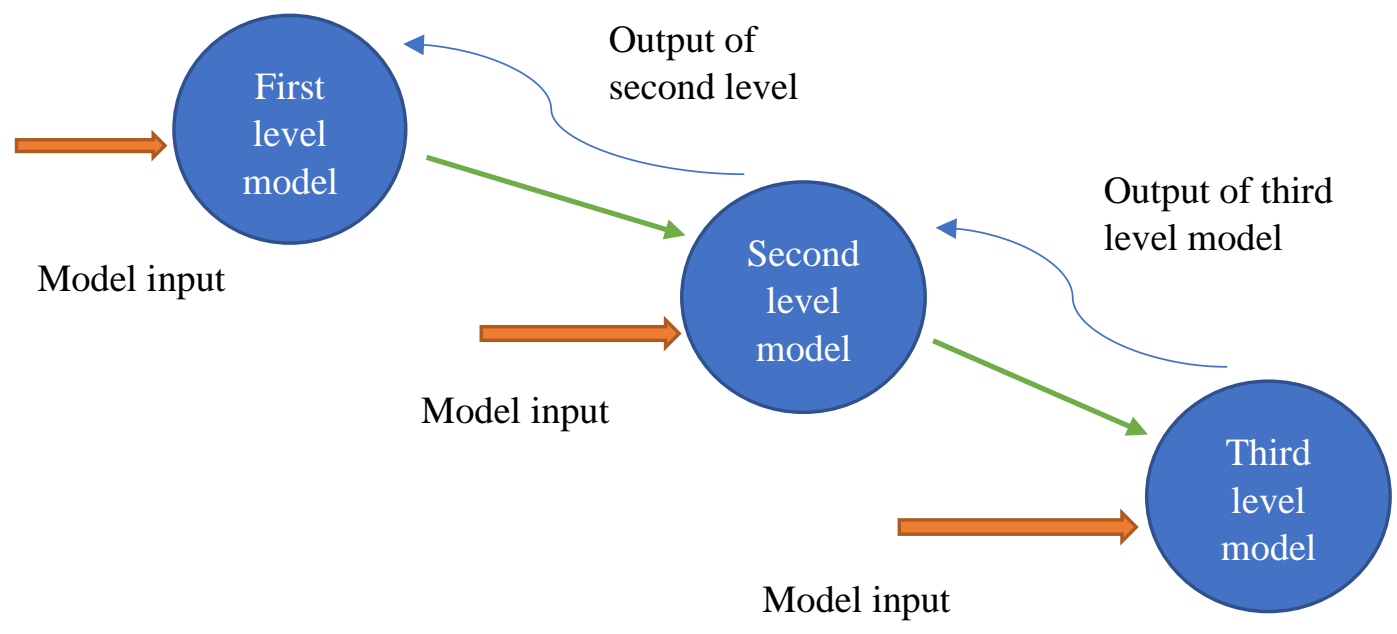

Figure 1.3: Hierarchical model type one

The second hierarchical planning type considers the various decision-making subjects at different levels as an integrated model. Although with this approach, the modeling and solving of the 
problem are getting more complex, if we can reach an accurate optimal solution, it's a global solution of the whole model. For instance, Puigjaner and Lainez (2008) provided an integrated model that optimizes strategic, tactical, and operational decisions. Figure 1.4 depicts this approach.

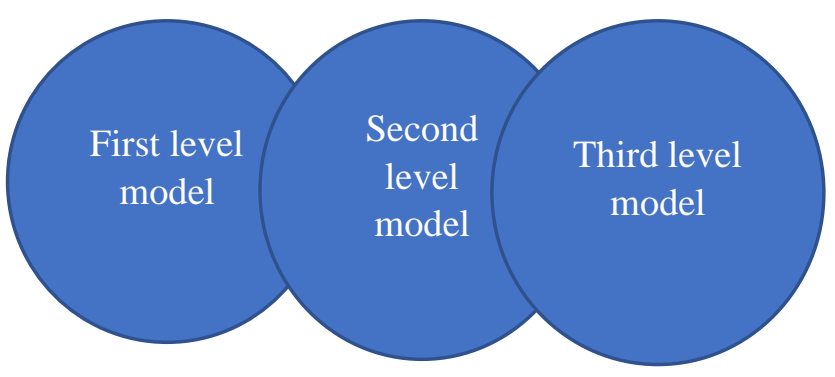

Figure 1.4: Hierarchical model type two

Finally, in the hierarchical planning type three, the second level model with having the output of the first level model solves parametrically, and then the output of this model puts in the first level model, which identifies the output of both models. For this type of model, the Stackelberg model is an excellent example in which the follower company finds its optimal production level by considering the production level of the leader as a parameter. Here, the follower's optimal level is the function of the leaders' optimal level. Then the leader puts the optimal production level of the follower in its model and gets its optimal production level. With having the leaders' production level, the followers' production level, which was a function of leaders' production level, is identified. Fig 3 illustrates this model.

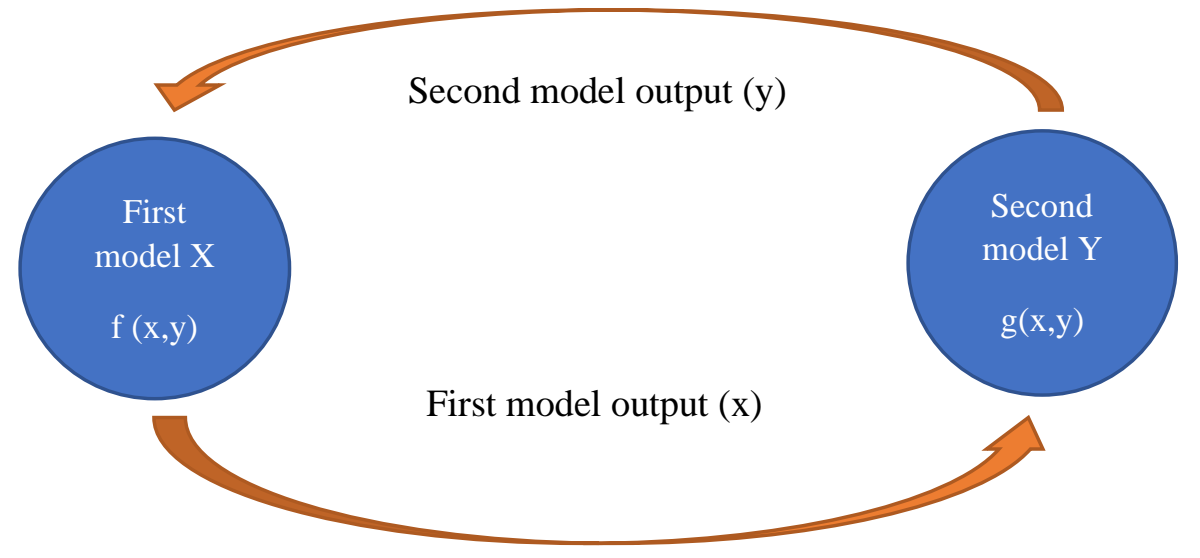

Figure 1.5: Hierarchical model type three

In chapters 3,4,5, and 6, hierarchical planning approach types one, two, and three are used and explained in detail how to benefit these models in joint venture integration and cooperation. 


\section{Contribution to the Iranian pharmaceutical supply chain}

We are beginning to see life-changing and curative medicines coming through development pipelines which are tremendously exciting, but with Iran's current pharmaceutical supply chain, getting access to modern and patent-based medicine is the biggest challenge for the patients. Patients with severe health conditions and their families have to take desperate measures to selffund medicines - selling their homes or using their life savings. The shortage of patent-based medicine and the high price are the main concerns for the Iranian government. Governments play an essential role in the pharmaceutical supply chain as they always try to reduce the cost of the national health care system and survive peoples' lives with the most effective medicines. In Iran, like other countries, the market of modern and patent-based medicine is under government control as the prominent legislator. Other external factors have contributed to the current disruptive medicine supply chain environment. The main reason is the international sanctions that have been imposed by the United States of America, making it challenging to import medicine. Money transfer, currency fluctuations, ordering cycle time, and supplier commitment are other consequences. The proposed model would be the best remedy for the Iranian pharmaceutical supply chain decision-makers at the macro level, putting the idea into the context of long-term strategies such as a coordinated approach to sharing intellectual property and technology transfer. The thesis model considers the market structure and the government's role as the leading participant of a strategic alliance and highlights the main parameters of the license contract to have the best agreement. The contribution of the model is also significant at the operational level too. Reducing order cycle time, improving the availability of the medicine, reducing the final price for the patients, and reducing the deterioration cost of the medicine for distributors can name as the most important contributions of the model.

\section{Supporting theories of a joint venture}

We can study joint ventures from seven different perspectives: Market-Power Theory (MPT), Transaction Cost Economics (TCE), Agency theory, Resource-Based Theory, Transaction Value Theory, Real option Theory, and Increasing Return Theory. These theories provide us valuable concepts in structure and process modeling of a joint venture that is described in detail as follows:

Market power theory considers how firms can be more successful in competition by maintaining stronger market positions. This approach is static and therefore focuses on how the textual, 
national, industrial, and organizational characteristics limit and shape partnership relationships at a particular time. The present study uses this theory to draw the joint venture perspective on changing market power.

The perspective presented by transaction cost economics sees strategic alliances as ways that can potentially reduce the cost of international trade. Transaction costs are imposed on a company when organizing, managing, and monitoring transactions in various markets, such as transaction costs, contracting, logistics management, and accounts receivable. The present study will use this theory to model the resulting synergy resulting from creating a joint venture alliance.

The ability of the employer to ensure that the agent meets the goals is an issue addressed in agency theory. From the agency theory, the following implication can be reached: As it is recommended that the employer uses a set of motivational measures and supervisory mechanisms to ensure that the behavior of the agent is consistent with the goals of the employer, it is equally recommended That the partners of a joint venture determine for the parties to the contract on what basis the return on capital is distributed among the partners and set up a system through which information is distributed fairly among all parties. The equitable distribution of joint venture revenue used in the present study, which determines the optimal parameters of a license agreement between partners, follows the agency theory.

The development of resource-based theory coincides with its focus on knowledge resources or a complex and well-established mix of knowledge and skills known as capabilities. These theories view companies as a collection of resources, some of which are available, some of which are specific to a particular industry or sector, and some of which are unique to a particular company. The organization must be able to upgrade its resources and capabilities simultaneously as changes occur in the external environment, and this is done either through internal development or through external resource-based learning. These resources may require additional resources to create a competitive advantage. Alliances are both a strategic resource and a complimentary resource for organizations. The following methods can achieve the acquisition of new resources. Internal development of resources if they have the necessary time and capabilities, outsourcing with the problem that it has a market-like mechanism and does not increase the tacit knowledge of the organization, the acquisition of other companies that have a cost equal to the profitability capacity of assets and also employees the key is likely to leave companies, and ultimately engaging 
strategies with the benefits of not being limited by market price, producing high-profit, low-cost goods, increasing flexibility, and tangible learning capabilities. The modeling of knowledge value, cost reduction, and synergy resulting from the partnership alliance used in the present study confirm the foundations of this theory.

The transaction value theory reflects transaction cost theory and is source-based. Source-based models focus on maximizing rents governing a portion of assets in a joint venture and fundamentally ignoring cost differences, and transaction cost economics theory focuses on minimizing transaction costs. The transaction value model suggests that the real issue is to maximize the common value in a corporate transaction and that if more transaction costs through a single asset increase revenue more, a corporate venture should be allowed to bear transaction costs, and lower rents may create higher net worth if exchanges decline. According to this theory, opportunistic incentives are possible only by achieving resource rents once, but long-term and value-added rents can be achieved through special partnerships based on efficient management and trust through joint ventures. In the present study, when a foreign company decides to form a partnership through a joint venture, it prefers to achieve large rents, i.e., penetration in the local market as a leader, over considerations such as technology protection, and hence the model presented in the research confirms this theory.

The real option theory in venture capitalists allows investors to defer entire investment and acquisition for a small fee (optional stock price) until risky external events occur, and when the results can be it was agreed that they would exercise their right to acquire and decide to invest or liquidate if the circumstances were to the contrary. In global markets, international investors view joint ventures as a bargaining chip, and when they are confident that things are going well, they turn to acquire a joint venture company. As can be seen, in many joint venture projects, the acquisition is very likely if successful. Therefore, modeling joint ventures in line with the possibility of dissolution if the goals used in the present study are not achieved is in line with the real option theory.

Economic theory has traditionally been based on factor data having a declining return after a certain point in time. However, some economists believe that bullish returns are very common in industries, especially knowledge-based ones. In such a situation, companies can gain more market share early, be able to retain their customers, and eventually capture the market without reducing 
returns (such as Microsoft). This phenomenon causes companies to establish a network with dense technologies and to unite to gather a group of companies and become a major market player so that competitors do not outperform them.

\subsection{The structure of the thesis}

The remaining of the thesis is organized as the following. In chapter 2, the literature is reviewed to identify gaps and research innovations in addition to understanding the related concepts used in the pharmaceutical supply chain. The following 4 chapters represent the thesis's proposed models in an order specified in the problem statement section of this chapter. Finally, the conclusions of the thesis as well as the future research opportunities, are highlighted in chapter 7. 


\section{Chapter 2: Literature Review}

\subsection{Introduction}

In this section, first, the studies related to the thesis are reviewed. Then, it is explained how the model of the thesis, the generalization of previous studies, could address the challenges in the research community under study, namely the pharmaceutical supply chain (PSC) of deteriorating medicines.

The literature review includes four main sections. The first section describes the PSC. Due to the importance of deterioration in the PSC, which imposes high costs to the supply chain, the deterioration-related studies are reviewed in the next section. The third section explains the strategies used for enhancing the performance of the PSC. Then, the proposed strategy of the thesis is briefly described. The strategy is about granting the production rights by a main manufacturer to a local manufacturer by forming a strategic alliance. To implement the strategy, a proper valuation mechanism is required to provide incentives for partners to follow the strategy. Hence, to interpret the strategy in the following chapters, a good understanding of the strategic alliance and valuation of intellectual properties, the pricing mechanisms in PSC to determine the profits of partners, and the coordination and profit distribution mechanisms should be achieved. These issues are explained in this chapter. The final section summarizes the reviewed studies and shows how the thesis innovations could cover the literature gap. This chapter's concepts provide the context of the proposed models and illustrate the tools and techniques that could be used to boost the thesis conclusions.

\subsection{The Pharmaceutical Supply Chain}

A PSC can include the following components: primary manufacturer, secondary manufacturer, distribution centers, and retail outlets, including pharmacies and hospitals. Figure 1.1 illustrated a general pharmaceutical network. The primary manufacturers produce the active ingredients, which are the chemical and molecular components of the medicine. Each stage of the production by these manufacturers such as quality control, changeover, and cleaning takes a long time which is the intrinsic characteristic of production by primary manufacturers. Secondary manufacturers mix 
excipients and active gradient compositions and then produce, package, and control the quality of the medicines. Distribution companies usually have a significant role in marketing the products by obtaining the necessary permissions. These companies are an integral part of the supply chain. Moreover, based on the purpose of a PSC, other players such as medicine inspection laboratories (Imran et al., 2018), managed care organizations (MCOs), healthcare maintenance organizations (HMOs) (Shah, 2004), and other governmental or administrative bodies could be included in the PSM. Usually, they are concerned with the issues of quality approvals or quality control processes.

On the other hand, governments impose more restrictions to control the price of medicines and are more likely to prescribe generic medicines in an attempt to manage the costly healthcare services in a better manner. Pharmaceutical companies are also willing to have the maximum share in markets during the terms of their patents. This can be justified by the short period of the patent term (8-12 years) as well as the high cost of introducing the product to the market $(\$ 200-400$ Million) (Grabowski, 1997). One of the highest costs of entering a market is the regulatory processes, such as the laws and regulations ratified by the U.S. Food and Medicine Administration, which has slowed the process of developing new products (Grabowski, 1997). There is also a growing trend in the movement of pharmaceutical companies from the local supply chain to the global supply chain, which has increased the need to develop logistic capacities (Booth, 1999).

In a pharmaceutical supply chain, operational issues can include demand management, distribution requirement planning, inventory management, and production scheduling. In this regard, there are also indicators for assessing supply chain performance, including stock levels in the whole chain, stock turns, inventory turnover ratio (annual sales/average inventory), supply chain cycle time, value-added time (production time/cycle time), material efficiencies (produced product/total material), etc. (Shah, 2004). Strategic topics in this supply chain are categorized as pipeline and development management, process development, capacity planning and plant and supply chain network design, factory design, issues of uncertainty in product development, and product demand (Shah, 2004).

In pharmaceutical supply chain research, many risk sources such as product failure during the trial, product withdrawal during sales due to side effects, uncertainties about final dosage and treatment regimens, and competition from similar products have been mentioned (Shah, 2004). Uncertainty in demand is another source of risk that has been investigated (Kelle et al., 2012). Researchers 
have proposed various indicators such as expected value, mean-variance (Mulvey et al., 1997), value at risk (Kall et al., 1994), and capital assessment pricing (CAPM) (Bhagwat \& Griggs, 1995) in order to consider risk.

Several studies have also been conducted on estimating the life span of pharmaceutical products. According to Shah (2004), before a medicine is available in the market, it undergoes about ten years of various tests, including a random test of effectiveness in R\&D centers. Afterward, patent protection can be applied to medicine. The medicine is then tested for safety and efficacy. This phase involves testing the harmlessness, non-toxicity, and potency of the medicine to lessen the symptoms and cure them. This process lasts 6 to 8 years and is known as developmental activity. Then, it is processed for production and distribution. This tremendous amount of effort and the short shelf life of patents have encouraged manufacturing companies to implement cooperative strategies to reduce costs and maximize market profitability. In the case of specific medications, as is considered in the modeling of this research, after the expiration of the patent term, there is the possibility of a change in the market structure because the medicine is being sold as generic. This could lead to a change from a perfect to oligopoly competition in the market structure, or in the case of several manufacturers and medicines, the structure is changed from oligopoly to perfect competition.

The health systems are characterized by high expenditure, high research and development cost, highly regulated structure, limited competition, and patient need dominance as one of the most basic human rights. The PSC accounts for a significant number of health system costs. In this regard, medicines were reported to form about $9.8 \%$ of all national healthcare expenditures in 2014 (National Center for Health, 2016). Moreover, due to the importance of the health system, governments in almost all countries, regardless of their income level, pay considerable attention to this sector of society and afford most of the medical costs through public insurances or other reimbursement schemes (Vogler \& Martikainen, 2015). Such trends reveal the desirability of the government in controlling and reducing the health system cost. It could establish an alliance with other players of the PSC and benefit from the resulting synergy of the cooperation. Doing so, the government could grant the rights and permissions of exclusive products to the producers and distributers of the PSC and have other partners discount the government by supplying the 
medicines at a lower price than the standard market price. Clearly, all the PSC partners, including the government, could improve their performance by engaging in such a strategy.

\subsection{Product deterioration in the pharmaceutical supply chain}

One of the most challenging issues in the pharmaceutical supply chain is product deterioration. The shelf life of medicine is defined as the period of time that the medicine's stability and functionality is over $90 \%$. The expiration date of medicine also indicates when the medication loses its effectiveness and safety (Coffey, 2013). In 2003, a survey in the United States showed that about $\$ 500$ million is lost annually due to the expiration of medications. In 2007 , the Health Department of Chicago lost about $\$ 1$ million because of the expiration of medicines (Masoumi et al., 2012). Another audit in the same year showed that pharmaceutical companies had about $\$ 2.05$ billion worth of medicines with expired dates. These pieces of evidence suggest that considering deterioration in the pharmaceutical supply chain models is of great importance. The strategies that are being developed to reduce the deterioration of products can result in a significant decline in deterioration, which benefits manufacturing companies and buyer organizations such as pharmacies.

Wang et al. (2011) modeled the relationship between retailers, distributors, and producers in the presence of deterioration. The integrated model of that paper explained how the supply chain partners could be affected by deterioration. To generalize the model, they considered the deterioration rate of products as a decreasing function of time, an increasing function of time, or a function independent of time.

Uthayakumar and Priyan (2013) studied the integration of inventory and production controls in a two-echelon supply chain, including a pharmaceutical manufacturer and hospitals as demand points. In that paper, demand uncertainty and delivery time were considered. Also, deterioration was modeled according to the medicines' expiration date, and the model was solved using the Lagrangian Relaxation Method.

In another study by Zahiri et al. (2018), the problem of designing a pharmaceutical supply chain was studied in terms of primary and secondary manufacturers, distributors, and demand points, as well as the costs and uncertain demands and deterioration of products, using the "interval-based 
robust possibilistic programming" (IRPP) approach and was modeled as a multi-objective problem. In that paper, the design of the logistics network of a supply chain was described as a research area that had been neglected by the researchers and therefore needed to be carefully reviewed. In that paper, the design of the network included decisions on locating, manufacturing, distributing, and allocating to the active facilities. Despite the modeling of product deterioration, pricing decisions were not addressed in that paper. Also, the supply chain's integration is introduced as an effective strategy to reduce the related cost of deterioration.

\subsection{Pharmaceutical supply chain strategies}

Pharmaceutical supply chain strategies have been proposed mainly to reduce costs, deterioration amounts, production and delivery times, and facilitate the communication between supply chain partners. The design and optimization of the pharmaceutical supply chain have attracted some researchers' attention in recent years. This is a strategic topic in the PSC and needs to be scrutinized as it affects the PSC's long-term performance. Some of the studies that addressed these strategies are reviewed in this section.

Nicholson et al. (2004) described and optimized a pharmaceutical supply chain for a potentially demanded product that was proposed as an optimal outsourcing strategy for unnecessary medicines. In that paper, using innovative algorithms, the optimal network was determined by comparing the existence of a $3^{\text {rd }}$ party logistic (3PL) for outsourcing in the network to the case of the only manufacturer and hospital.

Kim (2005) introduced an online logistics system. In that paper, the supply chain network included a number of pharmaceutical manufacturers, wholesale centers, and hospitals. The study showed that using an online system with the sharing of information between wholesalers led to the implementation of inventory management policies by the seller, and the results revealed that this policy reduced the cost of the supply chain.

Hassan et al. (2006) studied the improvement of a hospital's pharmacy network, taking into account various options, including space enhancement, pallet management, and warehouse automation equipment. In that research, after identifying decision criteria and weighing their 
importance, ELECTRE III, a multi-criteria decision-making technique, was used to prioritize the improvement of pharmacies.

Azzi et al. (2013) used the simulation technique to evaluate two logistics networks, direct logistics and indirect logistics, by considering a central hub. The central hub was introduced as a 3PL, and the results of this simulation indicated that the second model had a significant impact on reducing inventory-related costs. Moreover, it could deliver the medicines to hospitals at low shipping costs, small sizes, and less time, and is more optimal than the first model.

Balcázar-Camacho et al. (2016) proposed a centralized model for managing and controlling the inventory and distribution of pharmaceutical products in a network of suppliers, pharmacies, distributors, and patients. For this purpose, the supply chain was modeled as a linear model, and the GAMS software was used to solve it. In that paper, a centralized model with the flow of information between the network facilities as a cooperation mechanism was introduced, though this type of model was an integrated supply chain model wrongly introduced as a coordinated model.

Stecca et al. (2016) addressed the issue of inventory control and distribution of medicines in a logistic network, including a set of suppliers and pharmacies. Their paper proposed a mixed integer programming model, and the CPLEX program was used to solve the problem. Meanwhile, they assumed a central decision-making unit controls and manages the distribution and inventory program between the suppliers and pharmacies.

Imran et al. (2018) studied the problem of designing a pharmaceutical supply chain to select suppliers. The supply chain included suppliers and a quality assurance center for medicines and hospitals. In that paper, the quality of medicines was also examined by introducing an index of the number of complaints on total supplied products along with supply chain costs, and a fuzzy approach was used to consider the demand uncertainty.

\subsection{The strategic alliance and intellectual property valuation}

In this section, the strategic alliance concepts and their benefits are illustrated and the valuation methods of intellectual properties, which are substantial for determining the profit allocation between partners of the alliance, are investigated. 


\subsubsection{The strategic alliances}

Many contractual agreements between licensees and licensors appear in the form of strategic alliances. In the strategic alliance between two or more legally independent firms by a contractual agreement, partners' resources, skills, technologies, and experiences are collaboratively provided, and a temporary business for sharing profit and risks is established. Indeed, the strategic alliance has afforded many advantages for firms such as reduced transaction costs, learning, and knowledge sharing between firms (Zhang, 2007), economies of scale (Pape \& Schmidt-Tank, 2004), technology transferring (Girmscheid \& Brockmann, 2009) and overcoming economic or political barriers to entry into a new market (Sahebi et al., 2015). Despite the advantages of forming alliances, the strategic issues have been less discussed in the framework of mathematical models. In this regard, recently Hong and Chan (2014), in their review paper about construction joint ventures (CJVs) mentioned that "A retrospect of the observed publications on CJVs indicates a conspicuous lack of theoretical contributions to CJV study, ranging from building up a theory to developing CJV practice model and framework." Mellewigt and Das (2010) also underlined the necessity of a formula for the profit allocation problem in $J V$. Therefore, developing mathematical models of strategic alliances seems to be an emerging research topic, although it entails a great deal of effort to put the various assumptions of the problem in the real world into a mathematical model.

In the more recent studies, the benefits of participation have also been scrutinized. Bouncken et al. (2015) associated the emergence of cooperative alliances in response to the increase in the complexity, uncertainty, and competitiveness of business environments. Moreover, cooperation between firms has been mentioned to answer the changes such as shorter product life cycles, spiraling R\&D costs, risk sharing (Gnyawali \& Park 2009; Bouncken et al., 2015), and greater competitiveness (Alves et al., 2016). Some benefits of cooperation have also been enumerated such as access to technology, improved distribution channels, obtain synergies (Devece et al., 2019), staying agile and flexible (Park et al., 2014), gaining access to essential resources and knowledge (Bengtsson \& Kock 2000), resources and knowledge sharing, technical innovations by collaborated R\&D (Bengtsson \& Kock 2014), achieving economies of scale (Gnyawali \& Park 2011), economies of scope and access to new markets (Gnyawali \& Park 2009). 
On the other hand, one of the most important strategic topics in alliances is the division of profit between partners, namely the profit allocation problem (PAP). This problem has a significant impact on the performance of partners because it could affect their incentive for the success of the alliance (Parkhe., 1993). Some authors such as Poppo and Zenger (2002) and Reuer and Arino (2007) examined the link between the contractual agreement, cost, and performance of alliances.

To address the profit allocation in problems with low complexity, game theory approaches such as sequential bargaining models (Yan, 2012, Yan \& Yang, 2012), cooperative game theory based on the Shapley value (Sun et al., 2019), and core concept (Wu et al., 2017; Marzband et al., 2017) and competitive game theory (Müller \& Zaby, 2019) have been used. Moreover, for more complex problems in which the value of coalitions by different players or the value of different strategies of players could not be determined straightforwardly, other logical PAP mechanisms have been applied. For example, Moon et al. (2015) proposed that the generated profit of cooperation in supply chains could be split between the partners proportional to their profits before cooperation to the full profit of the non-cooperated supply chain. Hammani and Frein (2015) assigned the resulted profit of an integrated supply chain to partners based on their contribution in affording the supply chain cost.

To the best of our knowledge, few academic studies have been conducted on the profit allocation problem in alliances, and these scarce studies often paid attention to the determination of the factors that affect the profit allocation significantly. Their aim has been to present a theory about the factors affecting profit allocation or "pie-splitting" in a strategic alliance. Based on the proposed theories, a series of hypotheses have been tested using subjective judgments of experts or evidence in previous studies. For example, Contractor and Woodley (2015) found that the relative absorptive capacity of the partner, the existence of equity share, majority ownership, and high risks and benefits of the resulting income have a positive relationship with the relative share of the technology supplying partner, while relative proportions of tacit and codified knowledge and a number of minimum return provisions have a negative relationship. Howell (2018) advocated the need for a license contract that encourages opportunities for promotions of technology transfer between foreign firms and Chinese domestic companies by forming an alliance. He showed that, based on China's current industrial policies, foreign firms bring minimum technology due to substantial state ownership and high import tariffs contrast in China. So, a more 
flexible contract such as a license contract could facilitate technology transfer issues in countries with rigid trading laws. In a descriptive study, Katz et al. (1996) discussed the related issues of transferring technologies in global cooperative joint ventures. They investigated the types and methods of transferring technologies, the success rate of transferring, and what effects the differences in the culture, organization, and nationality of partners have on the transferring methods. These papers are beneficial in addressing the important aspects of the profit allocation problem. However, none of them have attended enough to develop a clear mathematical model or formula which facilitates the arguments between partners until they reach an agreement on a contract. Mellewigt and Das (2010) also underlined the necessity of a formula for the profit allocation problem in strategic alliances.

It is worth mentioning that, in the real world, the bargaining power of partners has a key role in PAP. Sometimes, the bargaining power is related to the value of intellectual properties (IP) owned by partners. Contractor and Woodley (2015) denoted this fact by focusing on the value of the technology supplied by one partner. Child et al. (2005) also stated that when two firms decide to form an alliance, first the value of the resources contributed to the alliance by each of them, tangible or intangible, should be carefully determined, and then, based on these values, the profit share of each partner will be assigned proportionally. In practice, the value of the tangible resources is widely calculated by some valuation methods or the market value known as goodwill.

It should also be pointed out that financing issues in the cooperative alliance have great importance. In this regard, shared finance sourcing might even be the cause or effect of forming some alliances (Skovsgaard \& Jensen, 2018). Alam et al. (2019), in survey research, found that non-alliance firms rely mainly on internal funds for their R\&D non-alliance, while in alliance firms, both types of internal and external funding are prevalent. Kang (2018) highlighted that the technological characteristics of pharmaceutical start-ups affect the decisions of financing sources such as angel, independent venture capital (IVC), and corporate venture capital (CVC). Drover et al. (2017) also reviewed the entrepreneurial equity financing studies, including Venture Capital, Corporate Venture Capital, and Angel studies. In that study, subjective judgment in decision-making processes, problems related to asymmetric information in financial markets, the value-added and performance outcomes for entrepreneurial firms, economic and behavioral antecedents of an alliance, decisions about engagement risk-sharing, etc., were categorized as problems of 
entrepreneurial equity financing studies. According to this reviewed paper, research about firms' intentions for joint investment beyond knowledge sharing should be considered.

\subsubsection{Intellectual property valuation}

Efficient management of the pharmaceutical supply chain to provide medicines efficiently at the right time will have a significant role in reducing the costs of the healthcare system and treatment of patients. Unlike many products, medicines are mainly produced by a limited number of manufacturers, and due to license issues as well as the high fixed costs of production, the import of medicines is widespread. Therefore, the lack of quick and timely access to medicines is one of the reasons for maintaining a large number of medicines, which imposes high costs on the supply chain. On this point, granting the production right through a license contract is a valuable strategy to reach the goals of semi-domestic production of medicines and overcome the problems caused by importing medicines. Although this strategy has not been cited explicitly as an effective solution to pharmaceutical supply chain problems, some studies have assessed the valuation of licenses in the pharmaceutical industry as intellectual property. In this section, we review some of this research.

Nicholas (1994) investigated the pharmaceutical supply chain investment using a stock-pricing approach and some other methods such as put options and call options. These methods make valuable assumptions, such as path-dependent ideas in pricing and forecasting the prices and demand rates, which can be used in many studies.

Some papers also attempted to create insights into the value of IPs in alliances. Sullivan (2000) discussed how intangible corporate assets could be converted into market value. Sercu (2009) proposed the proportional contract to value IPs through a license contract and related this value to the estimated cash flows of the sold product in the life cycle of products. They assumed the price and sale incomes are exogenous and known variables. Authors such as Longstaff (2001) and Schwartz (2004) also assume exogenous but uncertain sales income in their simulation-based model to discount the generated cash flows of patented products or value them. He used an American put option to rate a patented medicine. In that paper, the cash flows from investment in pharmaceutical products are identified using simulation, and then the average cash flow for different scenarios is presented as the value of the patent. The similarity of this assessment to the 
stock evaluation is that, in the evaluation of the patent, the project's continuation will not be economical if the future cash flow is less than the costs of the present period. In the stock market, the put option shares give the holder the option to sell their shares at a higher price than the current price if the prices fall.

Recent studies also paid attention to the IP valuation method from other viewpoints rather than valuing IPs. For example, Belderbos et al. (2014) showed that the co-ownership of patents with universities increases the value of patents while co-ownership and shared R\&D projects with other competitive firms shrink the value of the patent in a long-term horizon. Ko et al. (2019) proposed using a deep neural network to prepare a predictive model for transferability evaluation of intellectual properties. They determined various patent indicators related to patent transferability built a deep neural network by collecting data from various sources that map patent indicators into transferability citations with high precision. It is evident from the literature that patent valuation is mainly a technical and practical effort more than an academic one. The valuing models have developed in the context of finance research in the nineteenth century (Pitkethly, 1997). In fact, to value an asset, a great deal of effort is required to estimate different parameters that affect the perceived value of the asset. However, much more research is needed to incorporate other organizational issues into the valuing methods. In this regard, the proposed models of the thesis present the pricing decisions as endogenous variables that could be affected by the market's level of alliance capacity. The production capacity of the alliance in addition to the pricing decisions of the model, distinguish the proposed model from many other studies in this field.

\subsubsection{The pricing mechanism of pharmaceutical products}

Pricing decisions affect the throughputs of supply chains. If the final and intermediate prices of PSC items are correctly set, the partners' profitability and motivation could be ensured. However, the pharmaceutical supply chain and health care systems have different pricing mechanisms than other industrial sectors (Garattini et al., 2016). Ethical obligations of the medical community, essential medication needs of patients, high-cost pressure of patients in medicines preparation, affordability of medicines price, and the active and bold role of the government in reimbursement of patient cost are some reasons which make another prevalent pricing mechanism such as oligopoly or monopoly mechanism inapplicable. Therefore, policymakers have experienced and 
examined a number of pricing mechanisms in different countries, and some of them have been reported to be more persistent and efficient in providing sufficient and in-time medicines for patients and making the costs of the health system more affordable for governmental payers. The mechanisms mainly depend on the type of products. For medicines vital for the rescue and recovery of patients, the under-control pricing mechanism is more common. In these cases, the legislative agents have the most important role in pricing decisions. For other medicines and food products with relatively large producers, more competitive pricing mechanisms have been applied.

For under control-based pricing medicines, the three most applicable pricing mechanisms of pharmaceutical products include cost-based pricing, reference-based pricing, and value-based pricing mechanisms (Garattini et al., 2016). Cost-based pricing is based on estimating the sum of medicines' main cost items such as manufacturing, marketing, research and development, and administrative costs. This mechanism was prevalent in countries like Italy. However, transfer price difficulties resulting from the global nature of pharmaceutical industries made this mechanism obsolete in most countries (Garattini et al., 1994). The price of medicines in value-based pricing is identified such that the willingness of individuals in that price is maximized. Such prices reflect the perceived value of medicines for patients. This mechanism had been implemented in most Western EU countries since the 1990s. This is also faced with some economic disadvantages, especially in estimating the price of new medicines which are exposed to many uncertain events and assumptions (Drummond \& Sculpher, 2005). The most commonly used mechanism in pharmaceutical pricing is reference-based pricing which tied the price of medicines to their therapeutic benefits (Schneeweiss, 2007). In this mechanism, medicines are clustered according to shared attributes. Doing so, the costs of medicines in one cluster have slight differences from each other. Therefore, the domestic or foreign price of already introduced medicines in a cluster could act as a reference for new medicines listed in the cluster (Garattini et al., 2016). To make the pricing mechanism of PSC products more flexible, innovative pharmaceutical pricing agreements are emerging throughout the world. Some of these innovative agreements are the classification of new medicines approved at the European level according to their potential innovation e.g., one-tofive scale Amélioration du Service Médical Rendu (ASMR) in France) (Garattini et al., 2016), payback contract (Garattini et al., 2016), cap setting as a ceiling threshold for prices (Chen et al., 2019), coverage with evidence development, financial-based risk-sharing, and performance-based 
risk-sharing (Dunlop et al., 2018). Some authors, such as Petrou (2016), who outlined the costeffectiveness of tendering on prices, compared different price mechanisms' efficacy.

Besides the three most common pricing mechanisms, innovative pricing agreements have been growing in adoption in recent years. Dunlop et al. (2018), using the web-based survey, reported respondents' positive attitudes toward these agreements. They also argued that such a mechanism offers payers and pharmaceutical companies an opportunity to align on value, optimize speed to patients, and share risk.

For the less vulnerable pharmaceutical products such as the medicines' raw materials or foodrelated products, the competitive pricing mechanisms are applicable (Hokkanen et al., 2016; de Morais, 2017; Kaya \& Ghahroodi, 2018). In the competitive pricing mechanisms, the demandsupply relationship could be established.

In a study by Masoumi and Nagurney (2012), the pharmaceutical market was recognized as an oligopoly market wherein the Nash equilibrium specified the price of products. At that point, the supply chain costs and the graph theory were used.

As manufacturers of most medicines are limited to a few manufacturers, there is always an oligopoly market for pharmaceutical products (Ching, 2010; Masoumi \& Nagurney, 2012). There are different approaches to modeling the pricing structure of oligopoly markets. When the demand is fixed and only the demand's market share depends on some explanatory variables, Multi-Nomial Logit (MNL) and Constant Elasticity of Substitution demand functions have been used in many types of research. Nocke and Schutz (2018) discussed this type of demand function and proved its equilibrium price and its uniqueness. The application of this type of demand function can also be seen in the oligopoly market of pharmaceuticals considered in Kouvelis et al. (2015). They modeled the pricing of medicines and the discount in insurance firms' reimbursement schemes using this demand function in the game between pharmaceutical companies, insurance companies, and the government. The other mechanism, which considers the demand as a function of the price, is the Conjecture Price approach. In this approach, the equilibrium price is extracted utilizing the concept of players' best response and the conjecture of players about the effects of other players' actions on the equilibrium price. For example, this approach is used in the paper of Moiseeva et al. (2017) for addressing capacity planning, pricing, and the determination of capacity changes in the electricity market. 


\subsection{Cooperative game theory and the coordination mechanisms}

This section consists of two sub-sections. First, cooperative game theory and its application in the PSC are underlined. Next, the coordination concept in the supply chain is highlighted. We regard our pricing scheme and contract setting as the coordination mechanism that motivates supply chain partners with conflicting attitudes to work together.

\subsubsection{Cooperative game theory}

It has been shown that cooperative game theory has a remarkable ability to provide an appropriate framework for the alliance arrangement. Adegbesan and Higgins (2011) proposed a conceptual model for the intra-alliance value appropriation based on the concepts of cooperative game theory introduced in Adegbesan (2009). They tested their model hypotheses by using regression analysis, while their results approved the hypotheses. Yan and Hsueh (2009) extended the concept of Shapely value in cooperative game theory to the task allocation and partner selection problem in alliances. Their case study was about tunneling work intended to be completed by the cooperation of a foreign company and two local firms in Taiwan. In that paper, the foreign firm used the Shapely value concept to select its partners and allocate the project's tasks to them to maximize its profit in the coalition. San Cristobal (2012) also extended the concept of Shapley value to the problem of profit and cost allocation in a project which was done by the cooperation of several firms. He modeled the rewards or the partners' penalties by considering their contribution to the tardiness or earliness of the project. Ouenniche et al. (2016) analyzed public-private partnership projects as a static non-cooperative game of complete information and presented an ordinal game theory algorithm for finding an optimal generalized Nash equilibrium. The proposed proportional contract of the thesis for PAP is also based on the Shapley value, and this concept can also be extended to the cases in which more than two partners exist in strategic alliances.

Wright (2004) studied the pharmaceutical regulation problems in Australia. That game was implemented between the government as the regulator and the medicine manufacturers who want to supply medicines in the local market. The manufacturers who wanted to enter the regulation process, agreed on a price below the market price, and competed with all suppliers. The agreed price determination and decision about going through the regulation process were investigated using multi-stage game theory. 
Yan (2012) has used sequential bargaining models to investigate the $P A P$ in a joint venture alliance by considering the risk of failure to reach an agreement.

In the study by Rego et al. (2014), cooperative game theory was used to integrate orders of several hospital pharmacies to combine the compositions and utilize economies of scale. In that paper, a model was presented to provide the best combination of coalitions for hospital units to save transportation costs and inventory control. Due to the proposed model's complexity, the variable neighborhood structure method (VNS) was used to solve the problem.

Hou et al. (2016) studied a three-person game for investing in charging piles between local government, the State Grid Corporation, and clean-energy vehicle manufacturers in China. They showed that the three-party alliance yielded better outcomes than the one and two-partner alliance and proposed a cost-sharing mechanism to determine the share of parties in the investment.

Wu et al. (2017) used a cooperative game theory model based on the core method proposed to distribute the profit in a distributed energy network. In this network, the energy interchanges were used to overcome the supply-demand imbalance problem within a conventional distributed energy system.

The same problem was studied by Marzband et al. (2017), who considered the competitive pricing decision in addition to the profit distribution in a distributed energy resources system.

In Skovsgaard and Jensen (2018), a mixed-integer programming model is presented to find the optimal biogas value chain in biogas production plants. Then, the profit allocation mechanisms based on cooperative game theory are used.

Müller and Zaby (2019) utilized game theory to determine the best cooperation scenario, including no participation and only competition, no participation in R\&D but technology sharing and joint $\mathrm{R} \& \mathrm{D}$, and each strategy's profit for alliance partners.

The problems of entering a local market for a patent-holder when the government authorizes a compulsory license were investigated in Sarmah et al. (2019). They considered four stages for this arrangement. The first includes the research and development efforts done by the patent holder. The second stage was when the patent holder sold the new brand medicines exclusively. In the third stage, the license was granted to the local producers by the patent holder, subject to payment 
at the royalty rate per unit of sold medicines. The patent was expired at the fourth stage and the competition between the patent holder and the local producers continued without any payment to the patent holder. The equilibrium prices for each stage were determined in that study using the game theory approach.

The cooperative strategies for reducing the deterioration of medicines in the PSC, though it is a significant challenge, have been addressed in few studies. Those papers, such as Moslemi et al. (2017) and Abdulghani et al. (2019), mainly focused on the PSC's logistic capabilities and attempted to optimize supply chain operations by considering communications between PSC partners. Despite the remarkable models in the previous literature, the present thesis focuses on the cooperative strategy behind the integration and coordination of PSC operations. It extends the domain of the thesis to the valuing mechanisms which link the value of intellectual properties to their profitability and the benefits they create for companies.

\subsubsection{The coordination mechanisms}

One of the challenges in supply chain management is the management of individual partners that are dependent on supply chains. To achieve efficient performance in the supply chain, the partners must act as a coherent unit. The centralized control of the supply chain by a single decision-making authority that is fully aware of the entire chain and has the power to enforce the decisions can guarantee the optimal supply chain coordination (SCC). If there is a decision-maker in the supply chain, then the supply chain is said to have a centralized decision-making structure. In practice, however, there are different situations, and usually, there is no such decision-making center (Giannoccaro \& Pontrandolfo, 2004). On average, each supply chain is a set of independent economic entities, each of which decides based on personal profit and loss. If each partner of the supply chain has the power to make decisions, then it is said that the supply chain has a decentralized decision-making structure.

The main approach in moving from the decentralized decision-making mechanism is to make use of incentive schemes or coordination contracts when the supply chain is operated in a decentralized manner. Coordination models seek to find a feasible strategy to convince the independent partners of a supply chain to decide how the supply chain is centrally managed. In other words, coordination models seek to create a holistic view of the supply chain. Coordination mechanisms can be used 
to encourage the partners to make decisions according to the global optimum. In this case, a partner of the supply chain with a higher bargaining power tries to match the decision-making mechanism of other chain partners to the centralized optimal decisions. When a decentralized supply chain is managed in a centralized mode, the sum of all partners' profits is equal to the total profit of the supply; in such a situation, it is said that the supply chain is in perfect coordination (Cachon, 2004).

Supply chain coordination is a vast subject and covers various aspects of the relationship between the chain partners. In general, research in the field of supply chain coordination is divided into several general sections (Kanda \& Deshmukh, 2008):

The importance and role of supply chain coordination

$>$ Coordination in various activities of the supply chain such as product design and demand forecasting

Coordination in the relation between different parts of the supply chain such as purchasingmanufacturing, purchasing-inventory, and manufacturing-distribution

Mechanisms for creating coordination in the supply chain

\section{Empirical case studies}

One of the important aspects of supply chain coordination is the mechanisms for making coordination. To establish coordination in the supply chain, some mechanisms and methods should be used to motivate the partners of the chain to participate in the global supply chain optimization. In general, there are four mechanisms for establishing coordination, as presented in Figure 2.1.

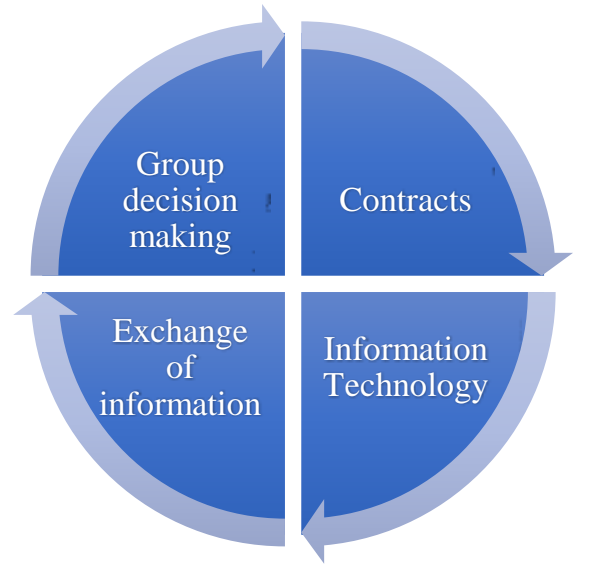

Figure 2.1 Four coordination mechanisms 
As previously stated, the centralized decision-making structure will result in better outcomes than decentralized structures. However, centralized decision-making results in worse outcomes for some partners of the supply chain, and, as a result, partners refuse to accept it. Incentive schemes are needed to prevent some partners' worsening conditions as a result of centralized decision making. The motivation for an economic entity is mainly in the form of higher profits. In other words, incentive schemes in the form of contracts can encourage the supply chain partners to make decisions in line with the centralized decision-making method. Moreover, contracts can be used as a mechanism for coordinating decisions in the supply chain. In brief, the contract in the supply chain is:

"A coordination mechanism that provides incentives to all of its partners so that the decentralized supply chain behaves the same as the integrated one" (Tsay, 1999).

So far, various contracts have been proposed for supply chain coordination. Some of these contracts will be included in the wholesale price contract: the buyback contract (Liu et al., 2014), the revenue sharing contract (Cachon, 2004), the quantity flexibility contract (Xiong et al., 2011), the discount contract (Sarathi et al., 2014) and the delay in payment contract (Duan et al., 2014).

Giannoccaro and Pontrandolfo (2004) studied the coordination of a three-stage supply chain that was based on a revenue-sharing contract by tuning its parameters.

Cachon and Lariviere (2005) studied the impact of revenue sharing contracts on the supply chain performance, which considered purchase quantity and price for each retailer while demand can be deterministic or stochastic.

Baboli et al. (2011) offered two models for centralized and decentralized supply chains. This supply chain consisted of a retailer and a wholesaler, and the cooperation between them could be centralized and decentralized. In the centralized model, the supply chain optimized the retail and wholesale costs, and their decisions were optimized in the centralized model, while in the decentralized model, each of the retailers and wholesalers optimized their models.

Zhao et al. (2012) studied designing a contract called FFS (Fee-for-Service Contracts) between distribution and production companies in the United States. It was argued that, prior to the execution of the contract, the policy of "investment buying" was common among the distributors, which led to the speculation of medicines because medicine prices were increasing every year, and 
distributors stored the needed medications in extra amounts to benefit from the increasing prices. On the other hand, FFS contracts have led manufacturers to closely monitor the distributors through information systems and pay wages for their services. Hence, with an analytical approach to calculating the yields of manufacturers and distributors, considering the potential demand, it is stated that the new contract should be written in such a way that the revenues of both the distributor and the retailer are more than the previous state, so they are motivated to execute the contract.

In the paper of Liu et al. (2014), the buyback contract is used as one part of the integrated model in which a vendor agrees to repurchase a part of the unsold product at the retailer's location for a certain price at the end of the sales season, accepting part of the overstock risk and persuading the retailer to order a larger amount of the product.

Govindan and Popiuc (2014) studied the impact of coordination in the two and three-echelon reverse supply chain. The results show how the revenue-sharing contract improves the performance measures and total supply chain profits.

Two new coordination mechanisms, namely economic and social collaborative model, were proposed by Nematollahi et al. (2017) in a PSC, including one retailer and one supplier. Their proposed model aimed to determine the visit interval of the supplier and the service level of the retailer in two ways: economic collaborative - maximizing the profit of PSC as a whole while ensuring minimum payoff (decentralized payoff) for all partners; social collaborative - assuming that managers will provide sufficient compensations though the decision making is done such that the social welfare of the patient is maximized.

Hosseini-Motlagh et al. (2018) proposed a supply chain coordination model with a manufacturer and two competing retailers. They showed that under different game structures and critical parameters, the proposed collaborative model could achieve coordination.

Taleizadeh et al. (2019) studied a reverse PSC in which the appropriate return prices could encourage customers to resell unexpired and useless medicines to the manufacturer. This initiative saves the environment from disposal damages of the medicines and provides additional income to the manufacturer by selling the returned medicines again at a reduced price. An almost similar problem when a 3PL was responsible for gathering returned medicines was considered (Weraikat et al., 2016). Revenue sharing was the coordination contract of that PSC. 
Khodabakhshi et al. (2019) coordinated a three-echelon supply chain comprising a manufacturer, distributor, and retailer with two coordination contracts: spanning and pair-wise revenue-sharing contracts. In that paper, the demand was a function of the retail price and the Corporate Social Responsibility (CSR) level.

Hosseini-Motlagh et al. (2019) investigated a two-level PSC, within which a manufacturer was in charge of CSR investment, and a retailer who faced a stochastic and scenario-based demand identified the retail price and order quantity of medicines. The problem was solved in two states of centralized decision-making and one decentralized.

The problem of price and quality setting in an SC comprising a manufacturer and packaging company was studied in Jabarzare and Rasti-Barzoki (2019). The problem was evaluated in three ways: a non-cooperative game, a cooperative game under revenue sharing, and a cooperative game through a profit-sharing contract. Their results highlighted the increase in the utility of the customers, especially in the case of profit-sharing contracts.

In the PSC, license contracts in various forms are mentioned to be a mediator for inter-firm partnerships. For example, Yoon et al. (2018) distinguished three types of license contracts milestone and optional upfront payment (royalty), optional upfront payment, and acquisition contract - to establish an alliance between a start-up firm and big pharmaceutical companies. They characterize the optimal arrangement for each contract and present insights about each contract type's benefits for start-ups and big pharmaceutical companies.

\subsection{Learnings from the literature}

Before comparing the present thesis with other studies in the literature, the studied strategies are briefly explained. Given that the present study examines the formation of strategic alliances in the supply chain of pharmaceutical products to reduce costs, two major cases are considered in the thesis. Also, considering that one of the most important decisions of strategic alliances is the way of cooperation and the partners' profit in that, and on the other hand, the distributed profit depends on the value creation of partners for the alliance, the main difference between the two cases is about the market structure in the two cases or their pricing mechanisms for estimating the profit of the alliance. 
The first case studies the medicines which act as the raw material of medications used by patients. The market for such medicines has more flexibility in determining the price. Moreover, the control of legislators is less due to the less susceptibility of patients to the medicines. In this case, determining the amount of investment in the alliance, the pricing decisions, and setting the cooperation contract parameters, which acts as the coordination mechanism of partners, includes the main topics. The main features of the proposed models in this case which distinguish this thesis from previous ones are dynamic pricing in an oligopoly or an exclusive market, the financing decisions, the decision about dissolving or continuing the company at any stage, decisions about tax and loans, and the high complexity of the model which entails a sophisticated solution approach.

The second case is about prescribing medicines that are mainly priced under the control of the government. In this case, given that most of the pharmaceutical supply chain costs are provided by the government, and through reimbursement plans, the government plays a key role in controlling the price and providing the necessary grants and permissions to pharmaceutical companies. On the other hand, due to the prominent role of technology in forming strategic alliances, the medicines studied in this case are brand medicines. In the case of generic medicines whose patent rights have expired, manufacturers can access the medicine formulation without forming a strategic alliance. The investigated issues, in this case, include the price arrangement in the current setting, the benefits of the alliance in reducing costs and deterioration of medicines throughout the supply chain, the modification of price based on the profit allocation to the partners as the coordination mechanism of the thesis and the parameters of the contract between main and local manufacturers.

Considering the above discussion, a comparison of the reviewed papers with the models of the thesis in the first case is presented in Table 2.1. Also, the second case models have been compared with some related papers in the literature as in Table 2.2. 
Table 2.1 A comparison of the first models of the thesis by reviewed studies

\begin{tabular}{|c|c|c|c|c|c|c|c|c|c|c|c|}
\hline \multirow[t]{2}{*}{ Article } & \multicolumn{3}{|c|}{ Strategic Decisions } & \multicolumn{3}{|c|}{$\begin{array}{l}\text { Operational and Tactical } \\
\text { Decisions }\end{array}$} & \multirow[t]{2}{*}{$\begin{array}{l}\text { Game } \\
\text { Theory }\end{array}$} & \multirow{2}{*}{$\begin{array}{l}\text { Multi- } \\
\text { Period } \\
\text { Models }\end{array}$} & \multirow[t]{2}{*}{$\begin{array}{c}\text { Profit } \\
\text { Allocation }\end{array}$} & \multicolumn{2}{|c|}{ Solution } \\
\hline & $\begin{array}{c}\text { IP } \\
\text { valuation }\end{array}$ & Financing & Coordination & $\begin{array}{l}\text { Capacity } \\
\text { Planning }\end{array}$ & Pricing & $\begin{array}{l}\text { Dissolve } \\
\text { Alliance }\end{array}$ & & & & Exact & $\begin{array}{l}\text { Heuristic } \\
\text { or Meta- } \\
\text { Heuristic }\end{array}$ \\
\hline $\begin{array}{l}\text { Nicholson et al. } \\
\qquad(2004)\end{array}$ & - & - & - & $*$ & - & - & - & $*$ & - & - & $*$ \\
\hline Wright (2004) & - & - & - & - & $*$ & - & $*$ & - & $*$ & $*$ & - \\
\hline Kim (2005) & - & - & $*$ & - & - & - & - & $*$ & - & $*$ & - \\
\hline $\begin{array}{l}\text { Hassan et al. } \\
\quad(2006)\end{array}$ & - & - & - & $*$ & - & - & - & - & - & $*$ & - \\
\hline Yan (2012) & & & & & & & & & & & \\
\hline Azzi et al. (2013) & - & - & - & $*$ & - & - & - & $*$ & - & - & $*$ \\
\hline $\begin{array}{c}\text { Balcázar-Camacho } \\
\text { et al. (2016) }\end{array}$ & - & - & $*$ & - & - & - & - & $*$ & - & $*$ & - \\
\hline Stecca et al. (2016) & - & $*$ & - & - & - & - & - & $*$ & - & $*$ & - \\
\hline Imran et al. (2018) & - & - & - & $*$ & - & - & - & - & - & $*$ & - \\
\hline Yan (2012) & - & - & - & - & - & - & $*$ & - & $*$ & $*$ & - \\
\hline Wu et al. (2017) & - & - & $*$ & - & - & - & $*$ & - & $*$ & $*$ & - \\
\hline $\begin{array}{l}\text { Marzband et al. } \\
\text { (2017) }\end{array}$ & - & - & $*$ & - & $*$ & - & $*$ & - & $*$ & $*$ & - \\
\hline $\begin{array}{l}\text { Skovsgaard and } \\
\text { Jensen (2018) }\end{array}$ & $*$ & - & $*$ & - & - & - & $*$ & - & $*$ & $*$ & - \\
\hline Yoon et al (2018) & $*$ & - & - & - & - & $*$ & - & $*$ & $*$ & $*$ & - \\
\hline $\begin{array}{l}\text { Müller and Zaby } \\
(\text { (2019) }\end{array}$ & - & - & $*$ & - & - & - & $*$ & - & $*$ & $*$ & - \\
\hline $\begin{array}{c}\text { Sarmah et al } \\
(2019)\end{array}$ & $*$ & - & - & - & $*$ & $*$ & $*$ & $*$ & - & $*$ & - \\
\hline $\begin{array}{l}\text { The first models of } \\
\text { thesis }\end{array}$ & $*$ & $*$ & $*$ & $*$ & $*$ & $*$ & $*$ & $*$ & $*$ & $*$ & $*$ \\
\hline
\end{tabular}


Table 2.2 A comparison of the second model of the thesis by reviewed studies

\begin{tabular}{|c|c|c|c|c|c|c|c|c|}
\hline \multirow[t]{2}{*}{ Article } & \multicolumn{2}{|c|}{ Strategic Decisions } & \multicolumn{3}{|c|}{ Operational and tactical Decisions } & \multirow{2}{*}{$\begin{array}{l}\text { Game } \\
\text { Theory }\end{array}$} & \multirow{2}{*}{$\begin{array}{l}\text { Deterioration } \\
\text { Strategy }\end{array}$} & \multirow{2}{*}{$\begin{array}{c}\text { Profit } \\
\text { Allocation }\end{array}$} \\
\hline & $\begin{array}{c}\text { IP } \\
\text { Valuation }\end{array}$ & Coordination & Pricing & $\begin{array}{l}\text { Replenishment } \\
\text { Cycle Times }\end{array}$ & $\begin{array}{c}\text { Order } \\
\text { Quantity }\end{array}$ & & & \\
\hline Giannoccaro and Pontrandolfo (2004) & - & * & $*$ & $*$ & $*$ & $*$ & - & - \\
\hline Cachon and Lariviere (2005) & - & $*$ & $*$ & $*$ & $*$ & $*$ & - & - \\
\hline Wang et al. (2011) & - & - & - & $*$ & $*$ & - & $*$ & - \\
\hline Baboli et al. (2011) & - & - & $*$ & $*$ & $*$ & - & $*$ & $*$ \\
\hline Zhao et al. (2012) & - & $*$ & - & - & $*$ & $*$ & $*$ & $*$ \\
\hline Uthayakumar and Priyan (2013) & - & - & - & $*$ & $*$ & - & $*$ & - \\
\hline Rego et al. (2014) & - & $*$ & - & - & $*$ & $*$ & $*$ & $*$ \\
\hline Liu et al. (2014) & - & $*$ & - & $*$ & $*$ & $*$ & $*$ & $*$ \\
\hline Govindan and Popiuc (2014) & - & $*$ & - & - & $*$ & $*$ & - & $*$ \\
\hline Weraikat et al. (2016) & - & $*$ & $*$ & - & $*$ & $*$ & - & $*$ \\
\hline Moslemi et al. (2017) & - & - & $*$ & $*$ & $*$ & $*$ & & - \\
\hline Nematollahi et al. (2017) & - & $*$ & - & $*$ & $*$ & $*$ & - & $*$ \\
\hline Nocke and Schutz (2018) & - & $*$ & - & $*$ & $*$ & $*$ & $*$ & $*$ \\
\hline Zahiri et al. (2018) & - & $*$ & - & $*$ & $*$ & - & $*$ & - \\
\hline Hosseini-Motlagh et al., (2018) & - & $*$ & $*$ & - & $*$ & $*$ & - & $*$ \\
\hline Abdulghani et al. (2019) & - & $*$ & - & - & $*$ & $*$ & - & $*$ \\
\hline Taleizadeh et al. (2019) & - & $*$ & $*$ & - & $*$ & $*$ & - & $*$ \\
\hline Khodabakhshi et al. (2019) & - & $*$ & $*$ & $*$ & $*$ & - & - & $*$ \\
\hline Hosseini-Motlagh et al., (2019) & - & $*$ & $*$ & $*$ & $*$ & $*$ & - & $*$ \\
\hline $\begin{array}{c}\text { Jabarzare and Rasti-Barzoki } \\
(\text { (2019) }\end{array}$ & - & $*$ & $*$ & $*$ & $*$ & $*$ & - & $*$ \\
\hline The second model of the thesis & $*$ & $*$ & $*$ & $*$ & $*$ & $*$ & $*$ & $*$ \\
\hline
\end{tabular}


Considering the subjects under study in Tables 2.1 and 2.2, the contribution of this thesis could be revealed. It could be said simply that the proposed models have a wider area than previous research by considering more assumptions and issues according to the real-world problems. More precisely, to highlight the contributions, the following points could be stated:

The proposed strategy of this thesis is different from the previous ones in several ways. First, it investigates the strategic issue of forming an alliance composed of supply chain partners. However, forming the alliance as a strategic issue has remarkable impacts on the operational as well as tactical issues of the supply chain. More precisely, the government as the main legislator of the PSC in granting the exclusive production right to domestic licensee manufacturers influences the pricing decisions and the market share of the research community's alliance. Second, the alliance set, which affects the profit distribution between partners effectively, is affected by sales and strategic alliance prices. Third, the alliance benefits other partners such as distributors and pharmacies in facilitating just-intime production and reducing the time between ordering, producing, and delivering the products. The latter is evident more in the second model of the thesis. Therefore, the proposed strategies complement previous individual strategies and prepare many benefits for the pharmaceutical supply chain.

Establishing domestic manufacturers that reduce the price of products, pharmacy inventory management, and the reduction of costs such as transportation and deteriorated products are the objectives of this thesis in line with the social welfare concepts of the supply chain. It should also be noted that all these measurements are based on the value-added activities which the governments or other social institutions promise. This accentuates the models' benefits compared with previous ones that studied cooperative strategies from few viewpoints.

The license valuation contract, which determines the terms of the contract and the licensor and licensee's profit, is based on cooperative game theory, which establishes a fair distribution of gain synergy to stabilize the long-term relationship of partners. Unlike other valuation methods, this takes into account the competitive advantages and competencies of all involved partners of the alliance. 
There are substantial differences between the pricing and valuing mechanism of the thesis with previous academic papers. For example, the price competition and license granting studied in the present thesis are different from those of Sarmah et al. (2019). Indeed, the medicine understudy in the current thesis is a brand medicine that owned some share in the local market, not a newly generated brand medicine. Besides, in contrast to Sarmah et al. (2019), the government has a determinant role in setting the allowed price of supplied medicines in the local market.

The profit allocation problem of the second model is more complicated than the reviewed ones. In that model, valuing the branded medicines in the negotiation process between the local manufacturer and the main manufacturer is based on the proportional contract of Sercu et al. (2008), which is according to cooperative game theory. However, because the profit of the government in the pharmaceutical supply chain could not be measured easily (the government just pays some portion of the patients' cost as its social responsibility) and the value of coalitions for different sets of players could not be estimated (for example when the government takes the responsibility of supplying medicines without the participation of the distributor), the profit distribution between the government, distributors, and pharmacies as well as the licensee local manufacturers is carried out proportional to their afforded costs of the pharmaceutical supply chain.

$>$ There are few studies that, in addition to valuing partnerships, have simultaneously examined financial issues. In one model of the thesis, the financing issues are studied, and the thesis extends the financing studies toward other objectives, which yield to the cooperation of firms.

$>$ In addition to the pricing decision, the present study considers pricing uncertainty to model the risk of investment. This innovation distinguishes the present research from valuation studies that are more based on past data in the estimation process. Determination of the optimum capacity is also not considered in any valuation models based mainly on simulation. Therefore, the combination of optimization models with simulation models is one of the innovations of the present study.

In some models of the thesis, the price competition in the pharmaceutical industry is investigated. Doing so, the price could be regarded as an endogenous variable, and the 
results of the model could be extended to another business context wherein the pricing decision is important for evaluating the efficiency of a business. However, the license valuation method of the thesis could be straightforwardly generalized to the other mentioned mechanisms by taking into account the price as an exogenous variable.

The proposed pricing mechanisms of the models in the second case are innovative pricing agreements for aligning the related bodies on the value created as the results of cooperation. These mechanisms regard the current price of medicines as a reference and modify the price so that the benefits of the PSC integration are taken into account. Therefore, this scheme does not neglect therapeutic values of medicines hidden in their current price and, after modification, leads to more profit for PSC partners than when they operate independently. For this purpose, cooperative game theory is utilized.

We also used the conjecture price approach to model pharmaceutical products' pricing decisions in an oligopoly market (first case). This is suitable for the proposed models in case 1 due to the following reasons: 1 ) it could handle the capacity planning decisions, and this issue is an important challenge addressed in this thesis. 2) given the capacity level, the equilibrium price in this approach could be determined by solving a quadratic mathematical programming model. 3) the parameters of this approach could be estimated using the market's historical data, which are available instead of the firm-specific or confidential data. 4) the considered medicine is homogenous, and for homogenous medicines, the most relevant determinant of the demand function is the price as regarded in the conjecture price approach.

There is a significant difference between the present research and the research by Masoumi and Nagurney (2012). In the present study, to model the oligopoly markets, we use "conjecture variation" which is very common in oligopoly markets and makes matters easier. On the other hand, as a strategic decision, we seek to find the optimal production capacity, and instead of evaluating the capacity of a single-period model, we need to evaluate the multi-period model. Moreover, given the long-term time horizon, one cannot ignore the uncertainty in the demand function results. In the present study, using the conjecture concept, we use firm-based planning to consider uncertainty. 
According to the above description, this study's cooperative strategy attempts to reduce costs and increase profitability and market share in line with real-world trends. The thesis' cooperative strategy also addresses the deterioration of medicines as a great concern in the pharmaceutical supply chain. This arrangement arises in the design stage of a pharmaceutical supply chain and plays an important role in estimating the development costs as a new firm's valuation model.

The license valuation framework of the thesis is based on game theory and resembles the coordination contracts that prepare sufficient incentives for the cooperation of the licensee and licensor. Another profit distribution mechanism could also be referred to as a coordination mechanism, which encourages PSC partners to work together instead of taking an isolationist approach.

The negotiation process of the thesis in a general case is structured in two stages. In the first stage, the main manufacturer negotiates with the domestic manufacturer to determine the license or license contract parameters' value by considering the price as an endogenous or exogenous variable. Upon completion of this stage, the profit of both licensor and licensee was determined using the Nash bargaining model. The same negotiation was done between the cooperative firm formed in the first stage and the government in the second stage. Examples of such arrangements are the papers of Nematollahi et al. (2017), Rego et al. (2014), and Li and Nagurney (2015). 


\section{Chapter 3: Pharmaceutical Alliance: Pricing and Profit Allocation Considering technology and Market Capabilities}

This chapter presents a novel formulation of the pricing and profit allocation (PAP) problem in a pharmaceutical alliance considering the license and other market capabilities such as the sales income, tax rates, inflation rate, and rates of return among alliance partners. A local firm $(L F)$ is considered decides to form a joint venture $(J V)$ with a foreign firm $(F F)$ to produce a pharmaceutical component locally by obtaining the required license from $F F$. Accordingly, there are some alternatives for $F F$ to give the $J V$ the license, including requesting some share in the ownership of the $J V$, receiving the license income from $L F$, or a combination of these two alternatives. Therefore, to maximize the two firms' gain and to obtain the optimal pricing decision of the $J V$, the considered $P A P$ problem is modeled by a contract called proportional contract. In this way, the synergy gain of cooperation is equally split between $L F$ and $F F$. Additionally, the chapter introduces a concept of marginal free-tax operational gain on the share $\left(M G_{S}\right)$ and successfully illustrates the interpretation of arriving at optimal decisions of the $J V$ using $M G s$. The results proved that if $M G s$ are positive, the maximum possible share of $F F$ on the ownership of the $J V$ is optimal. On the other hand, the negative $M G s$ stipulate the optimal $J V$ arrangement as a pure license contract. Notably, the results show that the standalone profit of the partners has significant effects on the alliance decisions, and therefore, the models also confer the best possible ways to determine the stand-alone profits for partners to agree on its value. Finally, the proposed framework is implemented in a real-world case study, and we show how different values of factual parameters affect the optimal decision through an in-depth sensitivity analysis.

\subsection{Introduction}

Most technology firms have developed their capabilities through alliances and acquisitions since the late 1990s ( $\mathrm{Li}$ et al., 2019). Firm alliance results are varied and can decrease or increase the value of a firm (Cabral \& Pacheco-de-Almeida, 2019). A joint Venture (JV) is a strategic alliance between two or more legally independent firms by a contractual agreement, wherein their resources, skills, technologies, and experiences are collaboratively provided to form a temporary business for sharing profit and risks. Indeed, the $J V$ has afforded many advantages for firms such as reducing transaction costs (Lee et al., 2015), learning and knowledge sharing between firms (Zhang, 2007), the economy of scale (Pape \& Schmidt-Tank, 2004), technology transferring (Girmscheid \& Brockmann, 2009) and overcoming economic or political barriers to entry into a new market (Sahebi et al., 2015). Despite the advantages of 
forming a $J V$, the strategic issues of $J V$ have been less discussed in the framework of game theoretical models. Therefore, developing practical and quantitative models of $J V$ seems to be an emerging research topic, although it entails a great deal of effort to put the different assumptions of the real world's problem.

On the other hand, as mentioned in the literature, one of the most important strategic topics in $J V$ is the division of profit between partners in a $J V$, the profit allocation problem $(P A P)$. This problem has a significant impact on the performance of $J V$ because it could affect the incentive of partners for the success of $J V$ (Parkhe, 1993). It should be noted that the previous studies, as reviewed in the literature, are beneficial in addressing the important aspects of the profit allocation problem. However, none of them have attended enough to develop a clear mathematical model or formula which facilitates the arguments between partners until they reach an agreement on a contract. The proposed proportional contract mechanism in this chapter could be valuable in providing a practical model for the profit allocation problem though this needs more development to cover all related issues in the problem.

It should be pointed out that the $I P$ valuation methods have some shortcomings that make them incompatible and unequal for $P A P$, especially in $J V$. In this regard, some important points can be regarded as below:

1) These $I P$ valuation methods consider all value of the additional generated profit for $I P$ owners and neglect the efforts of other partners. Therefore, such methods might be questionable, especially in a strategic alliance based on trust and, sometimes, long-term relationships. For example, Schwartz (2004) presented a patent valuation method such that the investment cost and the generated incomes of a patented project are simulated through differential equations. Then a method, like stock option valuation methods, has been applied to determine the patent value by discounting the simulated cash flows. In this way, other partners' efforts and capabilities, such as their market shares, managerial and political capabilities, etc., have been ignored.

2) The value of some resources is apart from common market value. This fact is reflected in the accountant documents as goodwill value. In $J V$ formation, reaching a consensus on such goodwill value is hard and an area of disagreement, mainly when partners are from different countries. 
3) The great required computational effort of such methods and the different value of the same $I P$ by a different method make $I P$ valuation challenging and an area of disagreement.

4) When different partners own more than one intellectual property, these methods are not able to distinguish the share of generating surplus related to each $I P$.

However, it is notable that the patent valuation methods are worthwhile because they are based on rich studies that reveal market behaviors. So, they can be used as a reference for the estimation of $J V$ outputs. For example, the controlled diffusion process for required investment proposed by Pindyck's model (1993) or the stochastic process of asset valuation in Schwartz and Moon (1996) is advantageous for estimations of $J V$ output regarding sources of uncertainty. Using the proportional contract mechanism of the thesis, some mentioned challenges could be reduced. This is based not only on the estimated cash flows of $J V$, which is common in the patent valuation method but also distributes the generated surplus between partners based on the game theory approach and therefore does not neglect any efforts of the partners.

In this chapter, like Sercu (2009), a contract called "proportional contract mechanism" is proposed for $P A P$, and it is shown how this mechanism could be used to address different decisions in the $J V$ arrangement. Moreover, we discuss that the concept of stand-alone profit $(S P)$ in the proportional contract should be carefully assessed based on the problem's assumptions. $S P$ stands for each partner's profit if they do their common business without cooperation with each other. Therefore, we first highlight the considered problem's assumptions and then relate SP to the partners' investment in JV by considering their return rate on the investment $(R O C)$.

In the profit mechanism of the thesis, which is based on the proportional contract, the contract terms (namely the royalty rate and the lump sum), the ownership share of the partners, as well as the operational price of $J V$ should be identified. As shown later, the mathematical model of the mechanism is a non-linear constraint optimization problem for which neither the closedform solutions nor the optimality conditions could be extracted explicitly and using the first and second-order conditions. As a contribution in this regard, several indicators are defined in this chapter that not only facilitates the extraction of the optimal solution but also provides a valuable economic interpretation of the optimal $J V$ structure that present valuable management insights about the strategic decisions of the $J V$ as well. 
In the financial contexts, the integrated joint venture refers to a strategic alliance for establishing of which, two or more firms perform a new collaborative business jointly by sharing their resources and capabilities and under the control of a central decision-making unit (which includes representatives of all involved firms) (Al-Emadi, 2010). However, when a project is implemented by several companies and each carries out one or more parts of the project without the control of the other members, the non-integrated joint venture is relevant (Al-Emadi, 2010). The strategic alliance under study is an integrated $J V$ in which the ownership structure of the JV and the profit of partners are predetermined by a license agreement. The present chapter attempts to specify how the license contract terms are determined. These terms are the ownership share of partners, royalty fee, lump sum, and the mechanism, based on which, the sales income of $J V$.

\subsection{Cooperative joint venture}

In this section, the concept of the proportional contract, license valuation, pure license contract, and general arrangement of $J V$ based on the proportional contract are discussed.

\subsubsection{The concept of proportional contract}

To describe the proportional contract, the arrangement of $J V$ is first declared. In this setting, there is a foreign firm $(F F)$ with technical capabilities and a local firm $(L F)$ with market capabilities intending to form a $J V$ alliance in the local market. There are several reasons for both parties to cooperate instead of doing business individually. Also, one of the most important incentives of the $L F$ for forming a $J V$ is to access the technological capabilities which could not reach them internally. Another reason might be the high cost of purchasing technological capabilities using common transactions in the technology market. In other words, the $F F$ could get access to the local market without affording high transaction costs by engaging in the $J V$ alliance.

The profit allocation problem between two firms is modeled as a two-player game, and the game aims to distribute the payoff of $J V$ between the players according to an equilibrium solution. A known license contract in the context of the international joint venture is "the proportional contract" introduced in Sercue (2009). The mechanism of this contract in distributing the profit of cooperation between members coincides with the standard solution of two-players games. The standard solution of the two-player games is the one that assigns equal division of the cooperation surplus for each player. Also, many solutions of cooperative games 
such as the Shapley value, the nucleolus, the Banzhaf value, the CIS (Centre of the Imputation Set) value for two-player games result in the equal division of surplus or the standard solution. However, the term "proportional" in the "proportional contract" might be confusing and in contrast with a known solution of cooperative games identified as "proportional sharing of the surplus" (Moulin, 1987). In fact, the "proportional sharing of the surplus" is a solution that distributes the surplus of cooperation to members proportional to their stand-alone income not equally. Nevertheless, the term "proportional contract" is in accordance with the name of the contract introduced in Sercue (2009) and here it is re-expressed as the main reference of the thesis. It is also notable that the equal distribution of surplus is according to the 50-50 rules applied to all international $J V^{\star} \mathrm{s}$ in the country under study and many other countries as stated in Sercue (2009).

In setting up the "proportional contract", the optimal arrangement of the contract which yields to the maximum profit for partners has been less discussed in the previous studies. Furthermore, for the real-world applications, the sale income concept and the stand-alone profit of members in Sercu (2009); which are regarded as exogenous variables; should be clarified precisely. These issues constitute the questions of the present study and we provide parametric solutions with salient managerial insights to answer them. Indeed, we explained how the answer to these solutions is depended on the ability of the members to extract profits from the $J V$.

Following the real license agreements, it is assumed that the $F F$ has alternatives to grant its technology as the royalty fee, lump sum, and shares in JV ownership (Sinha, 2001; Anand \& Kanna, 2000). The royalty fee is a percent of total sales asked by $F F$ for exposing its rare technology in $J V$. FF can also receive a fixed license fee as lump sum from $L F$ instead or in addition to the royalty fee. The sum of the royalty fee and the lump sum is referred to as "license income" in the proportional contract. Besides the royalty fee and the lump sum, FF could accept less license income to reach more share in the JVownership and consequently in the JV control. In this manner, the "dividend revenue" shows the income related to the $J V$ ownership. The contract between partners specifies the share of each partner in $J V$ ownership and the agreement over the license income.

If the partners agree on the proportional contract mechanism for $P A P$, then the parameters of the contract will be determined in such a way that the gain of the partners will be equal (Sercu, 2009). In this setting, the tax rate on the partners' income has an important role in the optimal decisions. As a base assumption, the tax rate on the license income is greater than that of the 
dividend income for the $F F$. This is done by the local government to encourage foreign firms to participate in $\boldsymbol{J} \boldsymbol{V}$ directly. All tax rates also are greater than zero and less than one. Moreover, keeping in mind some of the limitations associated with the considered real-life case study, the following assumptions are considered in the proposed model of this chapter:

1) The technology-providing partner $(F F)$, which is selected by the local partner, has no market share in the local country. Although this scheme provides more negotiation power for the local partner and results in more net income for the $L F$, there might be other restrictions in the real world that strengthen this assumption. In this regard, private or public firms in the developing country may not have enough power or resources to negotiate with the famous firms for forming an alliance. For example, in the 1960s, Italian firms emerged as a new entrant in the oil industry and formed $J V$ alliances with the national oil companies in the Middle East. Such alliances strengthened OPEC partners' bargaining power and made an evolution in the oil industry, which was captured by the British firms before that (Al-Emadi, 2010).

2) After the formation of the alliance, the structure of the market in the local country will be exclusive. The reasons behind this assumption might be the facilities provided by the local government to encourage $F F$ to do business with $L F$ jointly. This is in line with the mentioned benefits of $J V$ for partners that could not obtain the facilities without cooperating. Furthermore, a competitive pricing mechanism regarding a pricesensitive demand is used. This is applicable because the proposed model is not about prescribed medicines, which usually have special pricing mechanisms. The model was just applied to the specific raw materials used in the production of medicines. These materials are produced and supplied in bulk by a limited number of suppliers. Also, for the wide usage in the local country, an exclusive market is regarded for the product of the $J V$. In fact, the specialization of technology and the protection of the government from $J V$ prevents many manufacturers to enter the market of the product.

3) Regarding assumptions 1 and 2, it is confirmed that out of the $J V$, the $F F$ and the $L F$ have just opportunities to invest in their own business instead of the $J V$. This assumption will facilitate the calculation of partners' $S P$, which is used in the proportional contract and explained more in the following.

The notation below is used to interpret the proportional contract. All parameters in the chapter are practical ones represented in the country's laws or the firms' accounting statements. 


\section{Parameters Description}

$\begin{array}{ll}a, b & \text { The coefficients of demand function where } a \text { is the market scale (the maximum } \\ \text { possible demand) and } b \text { is the price sensitive coefficient. } \\ \tau_{L F} & \text { The tax rate on the dividend revenue of the } L F \\ \tau_{F F, 1} & \text { The tax rate on the license income of the } F F \\ \tau_{F F, 2} & \text { The tax rate on the dividend revenue of the } F F \\ v & \text { The variable cost of production } \\ F & \text { The fixed investment cost } \\ R O C_{F F} & \text { The } F F \text { 's rate of return on investment } \\ R O C_{L F} & \text { The } L F \text { 's rate of return on investment } \\ r_{F F} & \text { The inflation rate in the } F F \text { business } \\ r_{L F} & \text { The inflation rate in the } L F \text { business }\end{array}$

\section{Variables Description}

$p \quad$ The price of the $J V$ product

$L \quad$ The lump sum for the $F F$

$r \quad$ The royalty fee for the $F F$

$Q \quad$ The demand of the product

$\pi_{J V, L F} \quad$ The $J V$ profit of the $L F$

$\pi_{J V, F F} \quad$ The $J V$ profit of the the $F F$

$\pi_{S P, F F} \quad$ The $S P$ profit of the $F F$

$\pi_{S P, L F} \quad$ The $S P$ profit of the $L F$

$L P \quad$ The license income of the $F F$ in the $J V$

$\pi_{J V} \quad$ Free-tax profit of the $J V$

$O_{F F} \quad$ The ideal gain of the $F F$ in the $J V$

$O_{L F} \quad$ The ideal gain of the $L F$ in the $J V$

$\varphi \quad$ The share of the $F F$ in the $J V$ ownership

Considering the above notations and assumptions, the proportional contract is justified based on Eq. 1.3 (Sercu, 2009). Each side of this equation denotes the gain of cooperation for the $F F$ and the $L F$. However, the right-hand side of this equation is regarded as the objective function of the optimization model and the equation itself is considered as a constraint in the model to ensure the partners' agreement on the proportional contract.

$$
\pi_{J V, F F}-\pi_{S P, F F}=\pi_{J V, L F}-\pi_{S P, L F}
$$


We want to explore whether the contract parameters $r, L$, and $\varphi$ have effects on the partners' gain, and if so, what their appropriate value is. so, in all cases considered in this chapter, the maximization problem as below is studied:

$\operatorname{Max} \pi_{J V, F F}-\pi_{J V, L F}$

s.t.

$$
\begin{aligned}
& \pi_{J V, F F}-\pi_{S P, F F}=\pi_{J V, L F}-\pi_{S P, L F} \\
& 0 \leq r, \varphi \leq 1 \\
& L \geq 0
\end{aligned}
$$

where:

$$
\begin{aligned}
& Q=a-b \cdot p \\
& L P=L+r \cdot p \cdot Q=L+r \cdot p(a-b \cdot p) \\
& \pi_{J V}=(a-b \cdot p) p-v(a-b \cdot p)-F \\
& \pi_{J V, F F}=\varphi\left(1-\tau_{F F, 2}\right)((a-b \cdot p) p-v(a-b \cdot p)-F-L-r \cdot p(a-b \cdot p))+(L+r \cdot p(a-b \cdot p))\left(1^{(3} \tau_{F F, 1}\right) \\
& \pi_{J V, L F}=(1-\varphi)\left(1-\tau_{L F}\right)((a-b \cdot p) p-v(a-b \cdot p)-F-L-r \cdot p(a-b \cdot p)) \\
& \pi_{S P, F F}=\varphi \cdot R O C_{F F} \cdot F \\
& \pi_{S P, L F}=(1-\varphi) \cdot R O C_{L F} . F
\end{aligned}
$$

Accepting the proportional contract mechanism, it is reasonable to find the value of $r, L$, and $p$ such that the gain of the partners as the objective function of model $A(1)$ is maximized. Eq.3.2 shows the demand function of $J V$ product. Eq.3.3 displays the total license payment received by the $F F$, including the lump sum $L$ and a percentage of the total sales characterized by the coefficient $r$. Eq.3.4 presents the total profit of the $J V$ before taxation. Moreover, in Eq.3.5, the profit of the $F F$ is determined, including the payment on the license by the $L F$ and the accounting profit of the $F F$ based on its share in the $J V$ ownership. In Eq.3.6, the profit of the $L F$ is shown. It should also be noted that all the income terms are taxed at the appropriate rates. The tax structure of the thesis is based on Sercu (2009). Here, the profit is assumed to be positive, as in Sercu (2009). However, the general version of the model, which is a multi-period and considers accumulated profits and losses, could be scrutinized in future studies. In Eq.3.7 and Eq.3.8, the $S P$ of the $F F$ and the $L F$ out of the $J V$ have been presented, respectively. As we mentioned in assumption (3.3), these stand-alone profits are conditional to the intended investment of partners, which here are paid in the partners' businesses instead of the $J V$. 
To provide evidence for the stand-alone profit of partners that is a function of their investment in the $J V$, as shown in Eq.3.7 and Eq.3.8, we pay attention to the case where the product of the technology has a value equal to $v$ in the local market. Also, it is assumed that this value is the net present value of the ongoing income, which is obtained as a result of investment by the $L F$ $(\varphi=0)$. In this case, the Nash equilibrium payoffs of the bilateral game between the $L F$ and the $F F$ will be the payoffs of $\left(v-\pi_{S P, L F}\right) / 2$ for both partners (Grossman \& Hart, 1986). Ignoring the difference between the tax rates, this fact is reflected in Eq.3.1 by setting $\varphi, r=0$ , which results in $L$ being equal to $\left(v-\pi_{S P, L F}\right) / 2$. Now, if we consider any different value other than $\pi_{S P, F F}=0$ when $\varphi=0$, the equilibrium payoffs of the game will not be acquired.

Also, because the main aim of the models in this chapter is to provide a practical formula for the $J V$ arrangement, it is necessary to clarify the $S P$ value. There are some different rates for denoting the return on the firms' investment in the financial literature. Here, the return on capital (ROC) ratio is suggested for discounting the investment of the partner out of the $J V$. $R O C$ is calculated by dividing the net profit of a firm after tax by the invested capital, including assets and liabilities. By this definition, there is no need to apply the tax rate on the investment amount of the partners in Eq.3.7 and Eq.3.8. This rate seems to be an appropriate choice for $S P$ determination due to the following reasons: First, this rate is determined based on the financial statements of firms. So, if we accept the financial transparency in the statements, the partners could agree on it, both in its calculation and its inclusiveness. Second, when the aim is to compare the profitability of the firms fairly, this rate is more appropriate than another rate, such as ROI (Return on Investment), which is applied more for internal purposes. Finally, this chapter's proposed deterministic model does not consider any uncertain event in the evaluation of the $J V$ 's outcome. In this manner, the cost of capital $\left(K_{e}\right)$ is one of the most popular rates of return, which considers the riskiness of the firms.

It should be noted that in many similar studies in the context of the game theory, the standalone profit is usually regarded as an exogenous parameter. So, the introduced way of measuring the stand-alone profit is one of the contributions of the thesis. Summarizing the above discussion, the proposed stand-alone profit is valid for the following reasons: First, some papers such as (Grossman and Hart, 1986) confirmed the validity of this concept. Second, this idea is not uncommon, and by referring to companies that evaluate intellectual properties, one ratio that they require of the users is their return on capital (ROC) rate. As clearly explained in 
the text, this rate is taken from companies' published documents and is the most appropriate rate for foreign purposes. Third, this rate is one of the indicators of firms in the stock market to attract investors and accurately reflects the profitability of the company.

Furthermore, it is notable that there might be a difference between the inflation rate of the $F F$ $\left(i_{F F}\right)$ and the $L F\left(i_{L F}\right)$, and if this is the case, the returns of the local firm and the foreign firm are measured in two different scales. In such a situation, if the $R O C$ of the $F F$ in its business is denoted by $R O C_{F F}^{f}$, Eq.3.9 could be employed to convert the return of the foreign firm to a scale that is compatible with that of the local firm.

$$
R O C_{F F}=\left[\left(1+R O C_{F F}^{f}\right)\left(\frac{1+i_{L F}}{1+i_{F F}}\right)\right]-1
$$

In this chapter, the above equations are used to interpret different issues about $P A P$ in a $J V$ alliance. It should also be pointed out that the proposed model is just a generalization of the previous models by taking into account the operational pricing decision and in-depth analysis of the parameters of the proportional contract. So, it is not claimed that the proposed model outperforms previous ones but generalizes them.

With respect to the proposed contract, some points should be mentioned. First, the proportional contract and the equal gain are based on Sercu's (2009) book that deeply investigates the joint venture and other international agreements. We could not find any other references which address the joint venture contract and its problems like Sercu (2009). On the other hand, equal gain or simply a 50/50 contract is very common in international contracts, especially in the research community. The contract of the National Iranian Oil Company with Mitsubishi and other contracts of the Petrochemical Company and the Ministry of Health are among the evidence about the applicability of the model (Shafiei Khah \& Amiri, 2014). Also, the proposed model is just a generalization of the previous models by taking into account the operational pricing decisions and in-depth analysis of the parameters of the proportional contract. So, it is not claimed that the proposed model outperforms previous ones but it generalizes them.

\subsubsection{The pure license contract}

There are situations in which the $F F$ is unwilling to participate in a JV directly i.e, $\varphi=0$. In these situations, the $F F$ offers its patent right to the $J V$ as a pure license contract. For example, the $F F$ might be reluctant to bear the risk of a $J V$ or might not have any plan to participate in 
the control of a $J V$. Also, there may be some restrictions on doing business in the territory of the $L F$ for the $F F$. In such situations, the share of the $F F$ in the $J V$ is zero, i.e., $\varphi=0$. Therefore, Eq.3.1 is modified to Eq.3.10 in this case.

$$
\begin{aligned}
& (L+r \cdot p(a-b \cdot p)) \cdot\left(1-\tau_{F F, 1}\right)=\left(1-\tau_{L F}\right)((a-b \cdot p) p-v(a-b \cdot p)-L-r \cdot p(a-b \cdot p)-F) \\
& -\operatorname{ROC}_{L F} . F
\end{aligned}
$$

Eq.10 can also be rewritten as Eq.3.11.

$$
(L+r \cdot p(a-b \cdot p))=\frac{\left(1-\tau_{L F}\right) \cdot((a-b \cdot p) p-v(a-b \cdot p)-F)-R O C_{L F} \cdot F}{2-\tau_{F F, 1}-\tau_{L F}}
$$

Considering Eqs.3.5 and 3.11, $\pi_{J V, F F}$; as the objective function; is specified according to Eq.3.12:

$$
\pi_{J V, F F}=\frac{\left(1-\tau_{F F, 1}\right)\left(\left(1-\tau_{L F}\right) \cdot((a-b \cdot p) p-v(a-b \cdot p)-F)-R O C_{L F} \cdot F\right)}{2-\tau_{F F, 1}-\tau_{L F}}
$$

By this arrangement, $\pi_{J V, F F}$ is just a concave function of $p$. Therefore, its maximum value will be achieved by setting the first derivative of $\pi_{J V, F F}$ in Eq.3.12 with respect to $p$ equal to zero and solving the resulting equation. Doing so, the optimal value of $p$, denoted as $\left(p^{*}\right)$ will be according to Eq. 3.13.

$$
p^{*}=\frac{a+b \cdot v}{2 b}
$$

Moreover, by substituting $p$ * into Eq.3.11, we have Eq.3.14 for choosing $r$ and $L$ :

$$
L+r \cdot \frac{a^{2}-(b \cdot v)^{2}}{4 b}=\frac{\left(1-\tau_{L F}\right) \cdot\left(\frac{(a-b \cdot v)^{2}}{4 \cdot b}-F\right)-R O C_{L F} \cdot F}{2-\tau_{F F, 1}-\tau_{L F}}
$$

Each value of $r$ and $L$ satisfying Eq.3.14 and the constraints of A (3.1) are the optimal parameters of the contract, and there is no preference over them. Based on the above discussion, Proposition 3.1 is stated as below:

\section{Proposition 3.1:}

Using the proportional contract, the parameters of the pure license contract should be determined based on Eq.3.14. In this case, the optimal sale price $p^{*}$ will be according to Eq.3.13. This price will also maximize the free-tax profit of the $J V$ in Eq.3.4. 


\subsubsection{The general $J V$ arrangement and license contract}

In the general case, $F F$ and $L F$ should agree on the $J V$ contract in the term of shares in $J V$ ownership, lump sum, and royalty fee. In this case as before, Eq.3.1 is considered and rewritten as Eq.3.15:

$$
\begin{aligned}
& \varphi\left(1-\tau_{F F, 2}\right)((a-b \cdot p) p-v(a-b \cdot p)-F-L-r \cdot p(a-b \cdot p))+ \\
& (L+r \cdot p(a-b \cdot p))\left(1-\tau_{F F, 1}\right)-\varphi \cdot R O C_{F F} \cdot F \\
& =(1-\varphi)\left(1-\tau_{L F}\right)((a-b \cdot p) p-v(a-b \cdot p)-F-L-r \cdot p(a-b \cdot p))-(1-\varphi) \cdot R_{O C C_{L F} . F}
\end{aligned}
$$

Indeed, for forming the optimal $J V$ agreement, each partner's gain should be maximized by selecting the proper values for $r, L, p$, and $\varphi$. Again, it is proved that the best value of $p$ is the one that maximizes the free-tax profit of the $J V$, i.e., $p^{*}$ in Eq.3.13. So, in the general $J V$ arrangement, Proposition 3.2, whose proof has been presented in Appendix 3.1, is stated as follows.

\section{Proposition 3.2:}

In the general $J V$ arrangement, based on the proportional contract model, the optimal value of $p$ is the one that maximizes the profit of the $J V$ in Eq.3.4. (i.e., $p^{*}$ in Eq.3.13).

A valuable result of this section is that when the partners in $J V$ agree on the equal gain in the proportional contract, then the optimal value of $p$ will not be affected the ownership of partners in $J V$ and always will be such that the $J V$ profit in Eq.3.4 is maximized. In this regard, despite the claim of some researchers such as Contractor and Woodley(2015), one might imagine that after forming $J V$ and setting the value of $r$ and $L$, if the technology providing partner obtains the control and authority of the $J V$, then it could change the price such that its profit is maximized. In this case, the desired price for $F F$, i.e., $p_{2}$ will be as Eq.3.13.

$$
p_{2}=\underset{p}{\operatorname{argmax}} \varphi\left(1-\tau_{F F, 2}\right)((a-b \cdot p) p-v(a-b \cdot p)-F-L-r \cdot p(a-b \cdot p))
$$




$$
+(L+r . p(a-b \cdot p))\left(1-\tau_{F F, 1}\right)-\pi_{S P, A}=\frac{a \cdot r \cdot\left(1-\tau_{F F, 1}\right)+\varphi \cdot\left(1-\tau_{F F, 2}\right) \cdot(a-a \cdot r)}{2 b \cdot r \cdot\left(1-\tau_{F F, 1}\right)+\varphi \cdot\left(2 \cdot b(1-r) \cdot\left(1-\tau_{F F, 2}\right)\right.}
$$

However, choosing $p_{2}$ violates the agreement on the proportional contract, and therefore, this mechanism does not allow the partners to exploit the authority of the $J V$ for their self-interest benefits. The proposition also shows how the incorporation of the pricing decision affects the licensing agreement.

Knowing the optimal $p$ by Proposition 3.2, to find the optimal value of $L, r$, and $\varphi$, we first introduce the ideal gain function of the $F F$ and the $L F$ in Eq.3.17 and Eq.3.18, respectively.

$$
\begin{aligned}
& O_{F F}=\left(1-\tau_{F F, 2}\right)((a-b \cdot p) p-v(a-b \cdot p)-F)-R O C_{F F} . F \\
& O_{L F}=\left(1-\tau_{L F}\right)((a-b \cdot p) p-v(a-b \cdot p)-F)-R O C_{L F} . F
\end{aligned}
$$

$O_{i}(\mathrm{i}=F F, L F)$ denotes the gain of firm $i$ when its' capability is enough for carrying out the business alone, for example, when the $L F$ has the technology by itself without the need to pay the license costs or when the $F F$ has access to the local market without any extra tariff or other obligations. Considering $O_{\mathrm{FF}}, O_{\mathrm{LF}}$, and $L P$, the gain function of the $F F$ and the $L F$ is rewritten as Eq.3.19 and Eq.3.20, respectively.

$$
\begin{aligned}
& \pi_{J, F F}-\pi_{S L, F F}=\varphi \cdot O_{F F}-\varphi \cdot\left(1-\tau_{F F, 2}\right) \cdot L P+\left(1-\tau_{F F, 1}\right) \cdot L P \\
& \pi_{J V, L F}-\pi_{S L, L F}=(1-\varphi) \cdot O_{L F}-(1-\varphi) \cdot\left(1-\tau_{L F}\right) \cdot L P
\end{aligned}
$$

Therefore, when the equality of the partners' gain in Eq.3.1 is taken into account, $L P$ is determined by Eq.3.21.

$$
L P=\frac{O_{L F}-\left(O_{F F}+O_{L F}\right) \cdot \varphi}{2-\tau_{L F}-\tau_{F F, 1}-\left(2-\tau_{L F}-\tau_{F F, 2}\right) \cdot \varphi}
$$

Now substituting $L P$ into one of the partners' gain, we have an optimization model which is denoted by A (3.2) and is as follows:

$$
\begin{aligned}
& \operatorname{Max} Z(\varphi)= \\
& \frac{\left(O_{L F} \cdot\left(1-\tau_{F F, 2}\right)-O_{F F} \cdot\left(1-\tau_{L F}\right)\right) \cdot \varphi^{2}-\left(O_{L F} \cdot\left(1-\tau_{F F, 2}\right)-O_{F F} \cdot\left(1-\tau_{L F}\right)+O_{L F} \cdot\left(1-\tau_{F F, 1}\right)\right) \cdot \varphi}{2-\tau_{L F}-\tau_{F F, 1}-\left(2-\tau_{L F}-\tau_{F F, 2}\right) \cdot \varphi}+ \\
& \frac{O_{L F}\left(1-\tau_{F F, 1}\right)}{2-\tau_{L F}-\tau_{F F, 1}-\left(2-\tau_{L F}-\tau_{F F, 2}\right) \cdot \varphi}
\end{aligned}
$$


s.t.

$$
\begin{aligned}
& L P \text { in Eq. (22) } \geq 0 \\
& L P \geq 0
\end{aligned}
$$

Also, if the derivative of $Z(\varphi)$ with respect to $\varphi$ in model A (3.2) is denoted by $Z^{\varphi}(\varphi)$, Eq.3.22 holds:

$$
Z^{\varphi}(\varphi)=\frac{A \cdot \varphi^{2}+B . \varphi+C}{(D . \varphi+F)^{2}}
$$

where

$$
\begin{aligned}
& A=\left(2-\tau_{L F}-\tau_{F F, 2}\right) \cdot\left(O_{F F} \cdot\left(1-\tau_{L F}\right)-O_{L F} \cdot\left(1-\tau_{F F, 2}\right)\right) \\
& B=-2 \cdot\left(2-\tau_{L F}-\tau_{F F, 2}\right) \cdot\left(O_{F F} \cdot\left(1-\tau_{L F}\right)-O_{L F}\left(1-\tau_{F F, 2}\right)\right) \\
& C=\left(1-\tau_{L F}\right) \cdot\left(2-\tau_{L F}-\tau_{F F, 1}\right) \cdot O_{F F}-\left(\left(1-\tau_{F F, 1}\right)^{2}+\left(1-\tau_{L F}\right) \cdot\left(1-\tau_{F F, 2}\right)\right) \cdot O_{L F} \\
& D=-\left(2-\tau_{L F}-\tau_{F F, 2}\right) \\
& F=2-\tau_{L F}-\tau_{F F, 1}
\end{aligned}
$$

Now, to explain the necessary conditions for the optimality, the changes in the sign of $Z^{\varphi}(\varphi)$ should be considered. To do so, the roots of $Z^{\varphi}(\varphi)$ (the values of $\varphi$ which result in $Z^{\varphi}(\varphi)=0$, should be specified. If these roots exist, then they will be according to Eq.3.23.

$$
\varphi=1 \pm \frac{\sqrt{\left(\tau_{F F, 1}-\tau_{F F, 2}\right) \cdot\left(1-\tau_{L F}\right) \cdot G \cdot H}}{\left(2-\tau_{L F}-\tau_{F F, 2}\right) \cdot G}
$$

where

$$
\begin{aligned}
& G=\left(O_{F F} \cdot\left(1-\tau_{L F}\right)-O_{L F} \cdot\left(1-\tau_{F F, 2}\right)\right) \\
& H=\left(O_{L F} \cdot\left(\tau_{F F, 1}-\tau_{F F, 2}\right)-O_{F F} \cdot\left(2-\tau_{L F}-\tau_{F F, 1}\right)\right)
\end{aligned}
$$

Based on Eq.3.22 and Eq.3.23, we could explain the optimal conditions regarding different situations which might exist on $O_{L F}$ and $O_{F F}$. The first note is about the necessary and sufficient conditions of optimality. The conditions are stated in Lemma $\mathbf{3 . 1}$ as below, and its proof is presented in Appendix 3.2.

\section{Lemma 3.1:}

In the general $J V$ arrangement based on the proportional contract, the necessary and sufficient condition for the affordability of the $J V$ is $O_{L F} \geq 0$. In other words, if $O_{L F}$ is negative, the formation of the $J V$ is not economically feasible. 
Next, we analyze the possible situations for $O_{L F}$ and $O_{F F}$ as the following:

Situation 3.1: $\boldsymbol{O}_{\boldsymbol{F} F} \leq 0$ And $\boldsymbol{O}_{\boldsymbol{L F}} \geq 0$ :

In this situation, it is straightforwardly verified that the radicand in Eq.3.23 is negative. Therefore, there is no real root for $\varphi$. Clearly, $Z^{\varphi}$ is negative because the coefficient $A$ in Eq.3.23 is negative too. Hence, $Z(\varphi)$ in $A(2)$, is a decreasing function of $\varphi$ and this indicates that the optimal value of $\varphi$ i.e. $\varphi^{*}=0$. Replacing $\varphi^{*}=0$ in Eq.3.22, the optimal value of the license income $L P^{*}$ will be as Eq.3.24 and Proposition 3.3 is stated as below:

$$
L P^{*}=\frac{O_{L F}}{2-\tau_{L F}-\tau_{F F, 1}}
$$

\section{Proposition 3.3:}

When $O_{F F}$ is negative or equivalent when the $F F$ could get more benefit by investing in its business than in the $J V$, then the optimal $\varphi$ (i.e. $\varphi^{*}$ ) is equal to 0 . This indicates that the $F F$ should receive its profit in the $J V$ as just the license income, allow the $L F$ to invest in the $J V$ alone, and accordingly extract the maximum synergy of forming the $J V$. In this case, the license income is determined by Eq.3.24, and the $F F$ could specify $L$ and $r$ such that Eq.3.25 holds.

Situation 3.2: $\boldsymbol{O}_{F F} \geq 0, \boldsymbol{O}_{L F} \geq 0$ and $\left(O_{F F} \cdot\left(1-\tau_{L F}\right)-O_{L F} \cdot\left(1-\tau_{F F, 2}\right)\right)>0$,

In this situation, when $\left(O_{F F} \cdot\left(1-\tau_{L F}\right)-O_{L F} \cdot\left(1-\tau_{F F, 2}\right)\right)>0$, the term $H$ is negative and, therefore, the radicand in Eq.3.23 is negative too. This shows the non-existence of the real roots for $\varphi$ in Eq.3.23. We have shown the negativity of the radicand for this situation in Appendix 3.3. Moreover, the non-negativity of $A$ in Eq.3.23 indicates the increasing nature of $Z(\varphi)$ for $\varphi$ in model A (3.2). Hence, $\varphi^{*}$ is the maximum possible value of $\varphi$. This value is shown in Eq.3.25 and makes $L P=0$ in Eq.3.21. (Note that it is straightforward to show when $\left(O_{F F} \cdot\left(1-\tau_{L F}\right)-O_{L F} \cdot\left(1-\tau_{F F, 2}\right)\right)>0$, then $\varphi^{*}$ in Eq.3.25 is less than the root of the denominator 
of $L P$ in Eq.3.21, i.e., $\left.\left(2-\tau_{L F}-\tau_{F F, 1}\right) /\left(2-\tau_{L F}-\tau_{F F, 2}\right)\right)$. It is also clear that further increases in $\varphi$ than $\varphi^{*}$ lead to negative values for $L P$, which are not acceptable.

$$
\varphi^{*}=\frac{O_{L F}}{O_{L F}+O_{F F}}
$$

Furthermore, when $\left(O_{F F} \cdot\left(1-\tau_{L F}\right)-O_{L F} \cdot\left(1-\tau_{F F, 2}\right)\right)=0$, it is still proven that $\varphi^{*}$ in Eq.3.25 is optimal. This proof is presented in Appendix 3.4. Collectively, as a result of this situation, Proposition 3.4 is presented below:

\section{Proposition 3.4}

When $O_{F F} \geq 0, O_{L F} \geq 0$ and $\left(O_{F F} \cdot\left(1-\tau_{L F}\right)-O_{L F} \cdot\left(1-\tau_{F F, 2}\right)\right) \geq 0$, the maximum share of the $F F$ in the $J V$ ownership is optimal. This value has been shown in Eq.3.25 making the license income in Eq.3.21 equal to zero.

To interpret the optimality of $\varphi^{*}$ in Situation 3.2, some points should be reconsidered. It was stated in Proposition 3.2 that the optimal pricing decision of general $J V$ assessment is such that the tax-free profit of the $J V$ in Eq.3.4 is maximized. This profit is not subject to any tax or license payment. The same argument could be utilized to explain the optimality condition of $\varphi$

. For this purpose, if we divide the term $\left(O_{F F} \cdot\left(1-\tau_{L F}\right)-O_{L F} \cdot\left(1-\tau_{F F, 2}\right)\right)$ by $\left(1-\tau_{L F}\right) \cdot\left(1-\tau_{F F, 2}\right)$, a new concept is introduced, which we denote as the marginal free-tax operational gain on the share $\left(M G_{S}\right)$. The $M G_{\mathrm{S}}$ is identified as Eq.3.26, which could also be expressed as a function of the model parameters by considering $O_{F F}$ and $O_{L F}$ definitions in Eq.3.17 and 3.18, respectively.

$$
M G_{S}=\frac{O_{F F}}{\left(1-\tau_{F F, 2}\right)}-\frac{O_{L F}}{\left(1-\tau_{L F}\right)}=\left[\frac{R O C_{L F}}{1-\tau_{L F}}-\frac{R O C_{F F}}{1-\tau_{F F, 2}}\right] . F
$$

The $M G_{S}$ stands for the change in the free-tax operational gain of the $J V$ when the ownership is transferred from the $L F(\varphi=0)$ to the $F F(\varphi=1)$. Note that in the $M G_{S}$ definition, no license effect is considered, and it is presumed that the partners have access to all the required capabilities when they are the owners of the $J V$. This also means that if the ownership is transferred from one partner to the other, no gain is considered for the transferor. Moreover, each partners' gain is divided by its related tax to separate the gain from tax. Therefore, the 
$M G_{S}$ indicates the performance of the $F F$ ownership on generating the gain in the $J V$. Hence, considering the $M G_{S}$, when it is positive, increasing the share of $F F$ is optimal, and this will result in the maximum gain of $J V$. Moreover, in defining the $M G_{S}$ as the most important index of the proposed model of this chapter, there are no variables, and this could be used before finding the variable to interpret the required conditions about the formation of $J V$.

Situation 3.3: $\boldsymbol{O}_{\boldsymbol{F F}} \geq 0, \boldsymbol{O}_{\boldsymbol{L F}} \geq 0$ and $\left(\boldsymbol{O}_{\boldsymbol{F F}} \cdot\left(1-\boldsymbol{\tau}_{\boldsymbol{L F}}\right)-\boldsymbol{O}_{\boldsymbol{L F}} \cdot\left(1-\boldsymbol{\tau}_{\boldsymbol{F F}, 2}\right)\right)<0$

If this is the case, the term $H$ in the radicand of Eq.3.23 could be either positive or negative. If $O_{L F} \cdot\left(\tau_{F F, 1}-\tau_{F F, 2}\right)-O_{F F} \cdot\left(2-\tau_{L F}-\tau_{F F, 1}\right)>0$, the radicand will be negative. Therefore, similar to Situation 3.1, the optimal value for the share of the $F F$ is $\varphi^{*}=0$ and $L P^{*}$ will be as in Eq.3.24. On the other hand, it could be shown that when $O_{L F} \cdot\left(\tau_{F F, 1}-\tau_{F F, 2}\right)-O_{F F} \cdot\left(2-\tau_{L F}-\tau_{F F, 1}\right) \leq 0$, then $\varphi^{*}=0$ is again the optimal solution of this state. In Appendix 3.5, the proof of this is illustrated.

So $\varphi=0$ is the optimal solution of model A (3.2) in this state, and we have Proposition 3.5 as below:

\section{Proposition 3.5:}

When $O_{F F} \geq 0, O_{L F} \geq 0$ and $\left(O_{F F} \cdot\left(1-\tau_{L F}\right)-O_{L F} \cdot\left(1-\tau_{F F, 2}\right)\right)<0$, the optimal $\varphi$ is $\varphi^{*}=0$. The licensing income is determined by Eq.3.24 and the $F F$ could specify $L$ and $r$ arbitrarily such that Eq.3.24 holds.

The results for Situation 3.3 are also in line with the argument about the $M G_{S}$ explained earlier. Here, based on the definition of the $M G_{S}$ in Eq.3.26, the $M G_{S}$ is negative; therefore, the performance of the $L F$ ownership in generating a gain for the $J V$ is greater than that of the $F F$. So, to obtain the maximum gain for the partners, it is optimal to allow the $L F$ to get the ownership of the $J V$. The same discussion is also true for Situation 3.1. Summing up these discussions, Proposition 3.6 is stated for the general $J V$ arrangement as the following. 


\section{Proposition 3.6}

In the general $J V$ arrangement, when the marginal free-tax operational gain $\left(M G_{S}\right)$ is non-negative, then the optimal share of the $F F$ in the $J V$ should be according to Eq.3.25. This value is the maximum possible value and makes the license income equal to zero, while if the $M G_{S}$ is negative, then the performance of the $L F$ in generating the gain of the $J V$ is better than the $F F$. So the share of the $F F$ should be zero, and the license income should be based on Eq.3.24.

Based on Proposition 3.6, two conditions are considered for the ownership structure of the $J V$. The first one, which is referred to as "Licensee ownership in the proportional contract," regards no ownership for the licensor, i.e., the $F F$, and arises when the $M G_{S}$ is negative. Moreover, we call the second ownership structure of the $J V$ "shared ownership in the proportional contract" which is optimal when the $M G_{S}$ is positive. This structure considers the maximum possible ownership for the $F F$. In fact, in shared ownership in the proportional contract, all the license value is transferred to the share of $F F$ in the JV ownership.

In this section, it is shown that the profit function of the partners is a decreasing or an increasing function of $\varphi$. Therefore, if there are some restrictions on $\varphi$, such as the restriction of the maximum share of foreign firms in the local country or the inability to provide the required investment by the partners, then these results are true. For example, if Situation 3.2 occurs but the maximum possible investment for the $F F$ is $F_{\max }$, which is assumed to be less than $\varphi^{*} . F$, then the optimal $\varphi$ will be such that $\varphi \cdot F=F_{\max }$ holds. This is due to the increasing nature of partners' gain with respect to $\varphi$ in Situation 3.2.

\subsection{Case study}

We consider a joint venture formed by an Iranian firm and a foreign pharmaceutical company to produce vitamin A for 20 years. Vitamin A is used in medicines related to skin, acne, skin ulcers, and human and animal nutritional supplements. The required investment for the JV Company construction is estimated to be $\$ 88000$ per year to produce 15 tons of vitamin A per year. The variable cost of production will be $11.5 \$ / \mathrm{Kg}$, including power cost, labor cost, and the cost of another chemical compound. Also, the financial statements of the firms reveal $R O C_{L F}=0.35$ and $R O C_{F F}^{f}=0.3$. Considering $r_{L F}=0.14$ and $r_{F F}=0.04$, then $R O C_{F F}=0.425$, 
according to Eq.3.9. The tax rates of the firm include $\tau_{L F}=0.25, \tau_{F F, 1}=0.3$, and $\tau_{F F, 2}=0.25$. The estimated annual demand function is characterized by $a=37500$ (million ton), $b=750$ ( $\mathrm{kg} / \$$ ). In Table 3.1, the data of the case study are summarized.

Table 3.1 The parameters of the case study

\begin{tabular}{|c|c|}
\hline Parameter & Value \\
\hline$a$ & 37500 \\
\hline$b$ & 750 \\
\hline$\tau_{L F}$ & 0.25 \\
\hline$\tau_{F F, 1}$ & 0.3 \\
\hline$\tau_{F F, 2}$ & 0.25 \\
\hline$v$ & 11,5 \\
\hline$F$ & 88000 \\
\hline$R O C_{L F}$ & 0.35 \\
\hline$R O C_{F F}$ & 0.425 \\
\hline
\end{tabular}

Using Propositions 3.1, 3.2, and 3.3, the optimal price of the $J V$ is $p^{*}=30.75 \$ / \mathrm{kg}$. The value of $p^{*}, O_{L F}, O_{F F}$, and the $M G_{S}$ will be $23641.4,17041.4$, and -8800 , according to Eqs.3.17, 3.18, and 3.26, respectively. Based on the negativity of the $M G_{\mathrm{S}}$, the optimal share of the $F F$ is $\varphi^{*}=0$. Therefore, the value of the license income $(L P)$ is $15761 \$$ based on Eq.3.24. However, if the local firm is not able to pay all the value of the license income in the form of a pure license contract, then the $F F$ could receive this value by considering the royalty fee such that Eq.3.3 is satisfied. For example, if the $L F$ could afford $50 \%$ of the license income, i.e., $L=7880.5 \$$, then the royalty percent will be 0.01775 , i.e., $1.775 \%$ of the total sales income. In the same way, if the licensor, i.e., the $F F$ receives all the license income as the royalty fee, the royalty fee will be twice, i.e., $3.55 \%$. Such a royalty rate value is common in the Food or Pharma \& Biotech industries. For example, in Parr (2012), the median royalty rate of $5.1 \%$ in the Pharma \& Biotech industry and a median royalty rate of $2.8 \%$ for the food industry were reported. The considered product, namely vitamin A, could fall in both categories of food and Pharma \& Biotech products. so, its royalty rate based on the proposed model could not be far from real-world practices. Also, according to Eq.3.1, the JV synergy, as the objective function of the model, will be $11032.7 \$$. 
The $M G_{S}$ value of the case study is according to Situation 3.3 where the tax-free generating gain of the $L F$ is greater than the $F F$. Therefore, the optimal share of the $F F$ in the $J V$ ownership is zero. However, in this case, the $L F$ is able to provide about $50 \%$ of the required investment. Although the investment of the $F F$ is not optimal for the $J V$ in generating the gain, however, the positivity of $O_{F F}$ indicates that this $J V$ is a profitable business for the $F F$ too. Therefore, the $F F$ could accept to invest in the $J V$ up to $50 \%$, i.e., $\varphi=0.5$. Doing so, the license income of the FF will be 4814.28 \$, according to Eq.3.21.

We investigate what effects different values of the parameters have on the optimal solution. This is done through sensitivity analysis by changing the value of the considered parameter while the value of the other parameters will be fixed, and according to Table 3.1.

\subsubsection{Changes in $R O C_{F F}$}

As mentioned earlier, $R O C_{F F}$ is a parameter that is determined based on the financial statements of firms. However, when the local partner has a choice to select a foreign firm as its partner in a $J V$, it might consider the $R O C_{F F}$ value of firms to choose a partner that results in more profit for it. In this regard, some notes should be considered. First, $R O C_{F F}$ does not affect the optimal price of the $J V$ based on Proposition 3.2 and Eq.3.13. On the other hand, when the $M G_{\mathrm{s}}$ is negative, the optimal share of the $F F$ is zero, and the optimal license income is according to Eq.3.24, which does not depend on $R O C_{F F}$. This means that whenever $M G_{\mathrm{S}}$ is negative, the changes in $R O C_{F F}$ do not affect the profit of the $L F$. However, it should be noted that the $M G_{\mathrm{S}}$ in Eq. 3.26 is a decreasing function of $R O C_{F F}$. We denote by $R O C_{F F}^{B P}$ the value of $R O C_{F F}$, which makes the $M G_{S}$ equal to zero and acts as a breakpoint for changing the ownership structure. Hence, regarding Proposition 3.6, when $R O C_{F F}$ is less than $R O C_{F F}^{B P}$, then $M G_{S}$ is positive, the share of $F F$ will be according to Eq.3.25, and the license income is zero. On the other hand, when $R O C_{F F}$ is decreased while the $M G_{S}$ is positive, $O_{F F}$ will be increased, and based on Eq.3.24, the share of the $F F$ is decreased. Fig. 3.1 shows the share of the $F F$ for different values of $R O C_{F F}$ in the considered case study. 


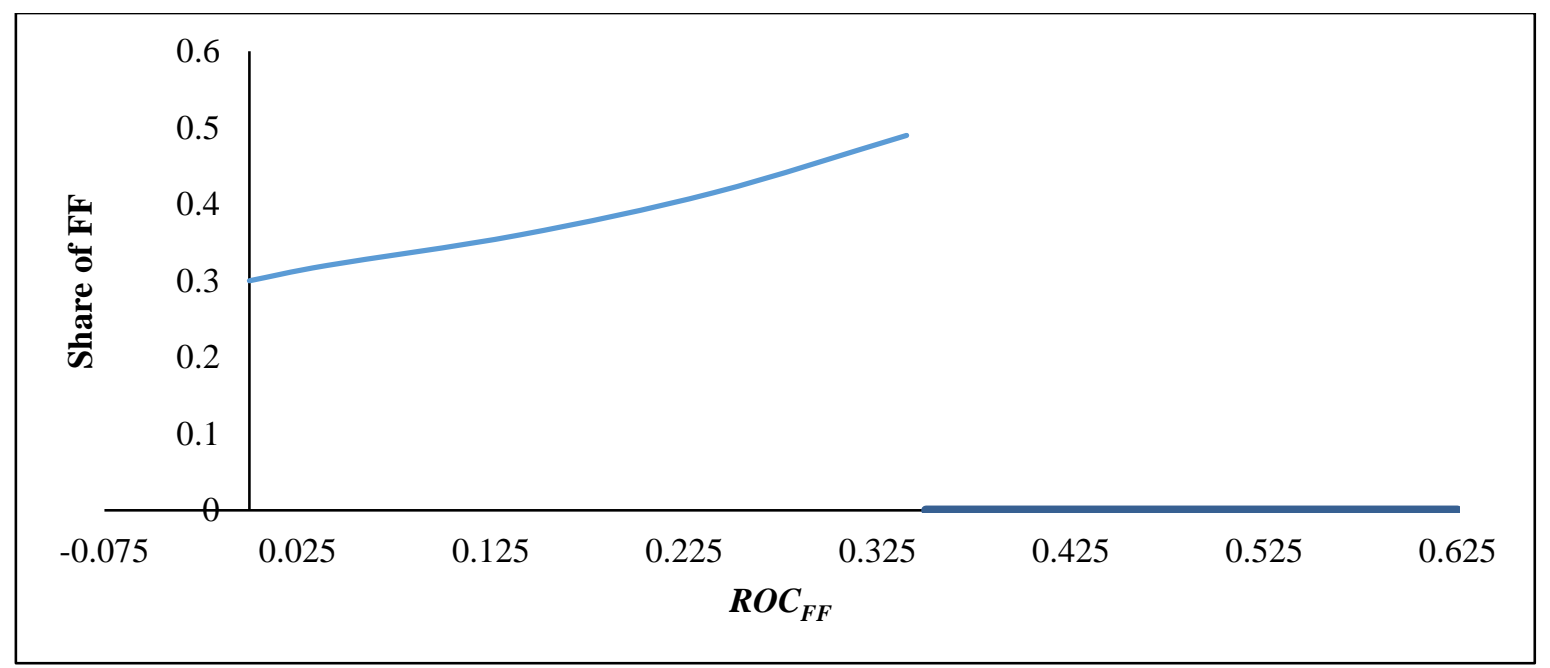

Figure 3.1 Share of the $F F$ vs $R O C_{F F}$

Based on Figure 3.1, if $R O C_{F F}$ is higher than 0.35, Licensee ownership is the optimal structure of the $J V$ wherein no share is considered for the $F F$. Also, the value of $R O C_{F F}$ less than 0.35 results in the shared ownership structure, and as mentioned earlier, the share of the $F F$ is decreased by reductions in $R O C_{F F}$. It is worth mentioning that a decrease in $\varphi$ will increase the profit of the $L F$ in the shared ownership structure. This is because there is no license income in a shared ownership structure, and $R O C_{F F}$ only affects the share of the $F F$ on its profit function. So, considering the optimal share of the $F F$ as Eq.3.25, the profit of the $L F$ will be a descending function of $R O C_{F F}$ in the shared ownership structure. The effect of $R O C_{\mathrm{FF}}$ on the profit of the $L F$ (Eq.3.6) is presented in Figure 3.2.

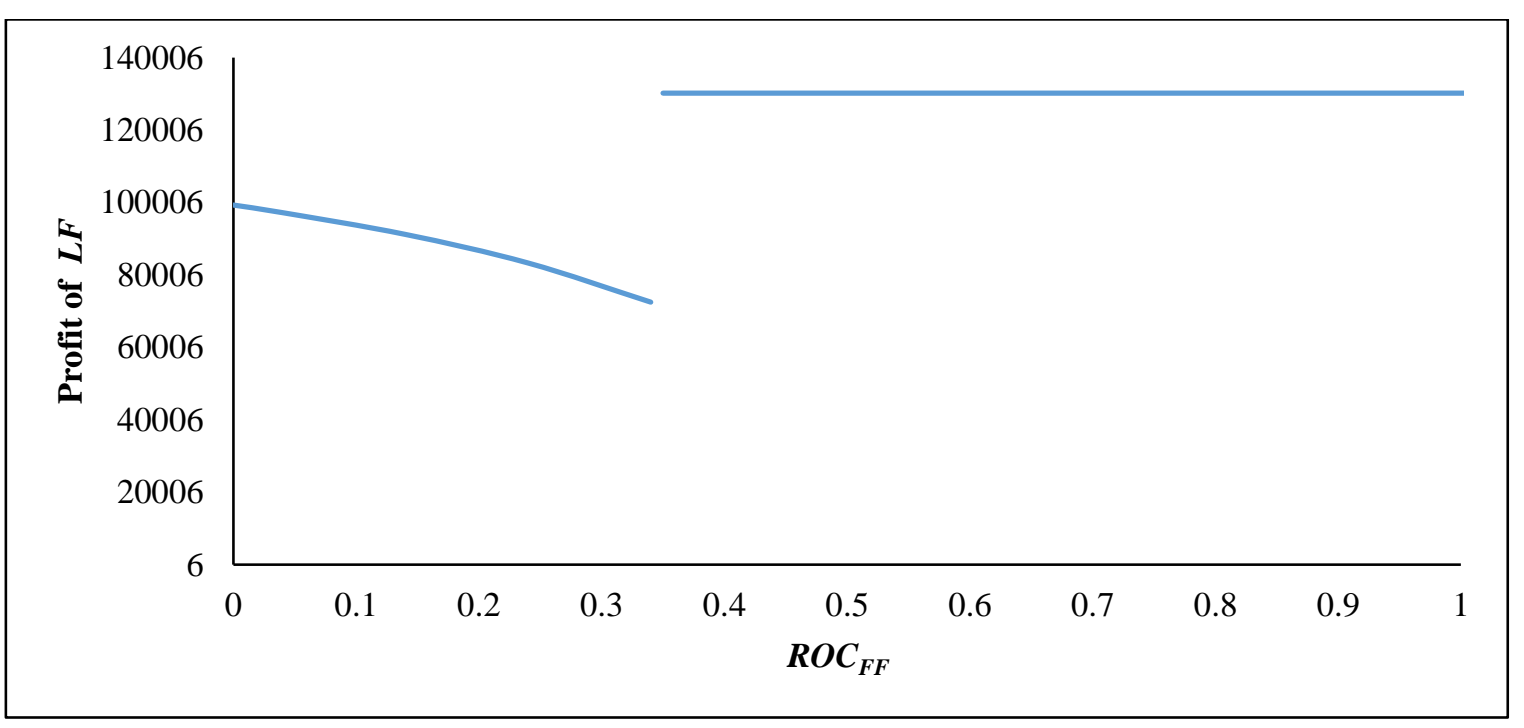

Figure 3.2 Profit of the $L F\left(\boldsymbol{\pi}_{\mathbf{J V}, \mathbf{F F}}\right)$ vs. $R O C_{F F}$ 
In Figure 3.2, when $R O C_{F F}$ is high enough to make the $M G_{S}$ negative and consequently leads to the structure of Licensee ownership, the profit of the $L F$ is the highest. So, in the proposed case study, the best choice for the $L F$ is to select a technology-providing firm with $R O C_{F F}$ equal to or bigger than $R O C_{F F}^{B P}$ (in this example, $R O C_{F F}^{B P}=0.35$ ). This result is in line with the base assumption of the model, which relates the stand-alone profit of the partners to the amount of investment that they bear in the $J V$. Doing so, whatever $R O C_{F F}$ is higher, receiving the income of the $J V$ as license income for the $F F$ is more profitable than participating in the ownership of the $J V$. On the other hand, ownership of the $J V$ by the $L F$ provides the maximum gain for it when $R O C_{F F}$ is changeable. So, as a valuable result, we present Proposition 3.7 as the following. The proof of Proposition 3.7 is shown in Appendix 3.6.

\section{Proposition 3.7:}

When the $L F$ has choices from which to choose its technology-providing partner in the $J V$, and the candidate partners agree on the proportional contract, each of the partners with $R O C_{F F} \geq R O C_{F F}^{B P}$ is an optimal choice and provides the maximum profit for the $L F$.

\subsubsection{Changes in the market scale parameter $(a)$ and the price-sensitive coefficient $(b)$}

The coefficients of $a$ and $b$ represent the structure of the market. The market structure has a vital role in the profitability of firms when they enter a market. In this manner, firms could obtain remarkable profit in some market structures, while in other markets, firms could not make a considerable profit as a result of high fixed and variable costs of production. Therefore, in this section, we investigate the effects of changes in $a$ and $b$ on the $J V$ arrangement and even $J V$ formation decisions. First, we consider the changes in the market scale parameter, i.e., $a$. In Proposition 3.6, it was declared that the sign of the $M G_{\mathrm{S}}$ determines whether the optimal share of the $F F$ in the $J V$ is zero or at its maximum possible value. Furthermore, by considering the $M G_{\mathrm{S}}$ as Eq. 3.26, it is easily verified that the derivative of the $M G_{\mathrm{S}}$ with respect to $a$ is zero. Consequently, a change in $a$ does not change the ownership structure of the $J V$, though it will change the share amount of the $F F$ if the $M G_{S}$ is positive. Moreover, when $a$ is decreased, the optimal price of the $J V$ in Eq. 3.13 is decreased, which reduces the demand and leads to an additional decrease in the sales income. Hence because the sales income is 
decreased, both the $O_{F F}$ and the $O_{L F}$ are decreased, and this reduction continues until $O_{L F}$ becomes negative. If this occurs, the formation of $J V$ is not economically feasible based on

\section{Lemma 3.1.}

To sum up the discussion, the changes in $a$ do not affect the ownership structure of the $J V$, yet alters the share amount of the partners. Moreover, when $a$ is decreased until $O_{L F}$ becomes negative, the formation of a $J V$ is not affordable anymore. Considering the case study in Table 3.1, $\pi_{J V, F F}-\pi_{S P, F F}$ and $\pi_{J V, L F}-\pi_{S P, L F}$ with respect to $a$ as depicted in Fig. 3.3. Furthermore, to show the effect of $a$ on the share of the $F F$, we turn our attention to the case where $R O C_{\mathrm{FF}}=0.34$, which results in a positive value for the $M G_{S}$. Then we show in Figure 3.4, how different values of $a$ affect the share of the $F F$. In both figures, the red line on the horizontal axis corresponds to the value of $a$ at which, the formation of a $J V$ is not economically feasible.

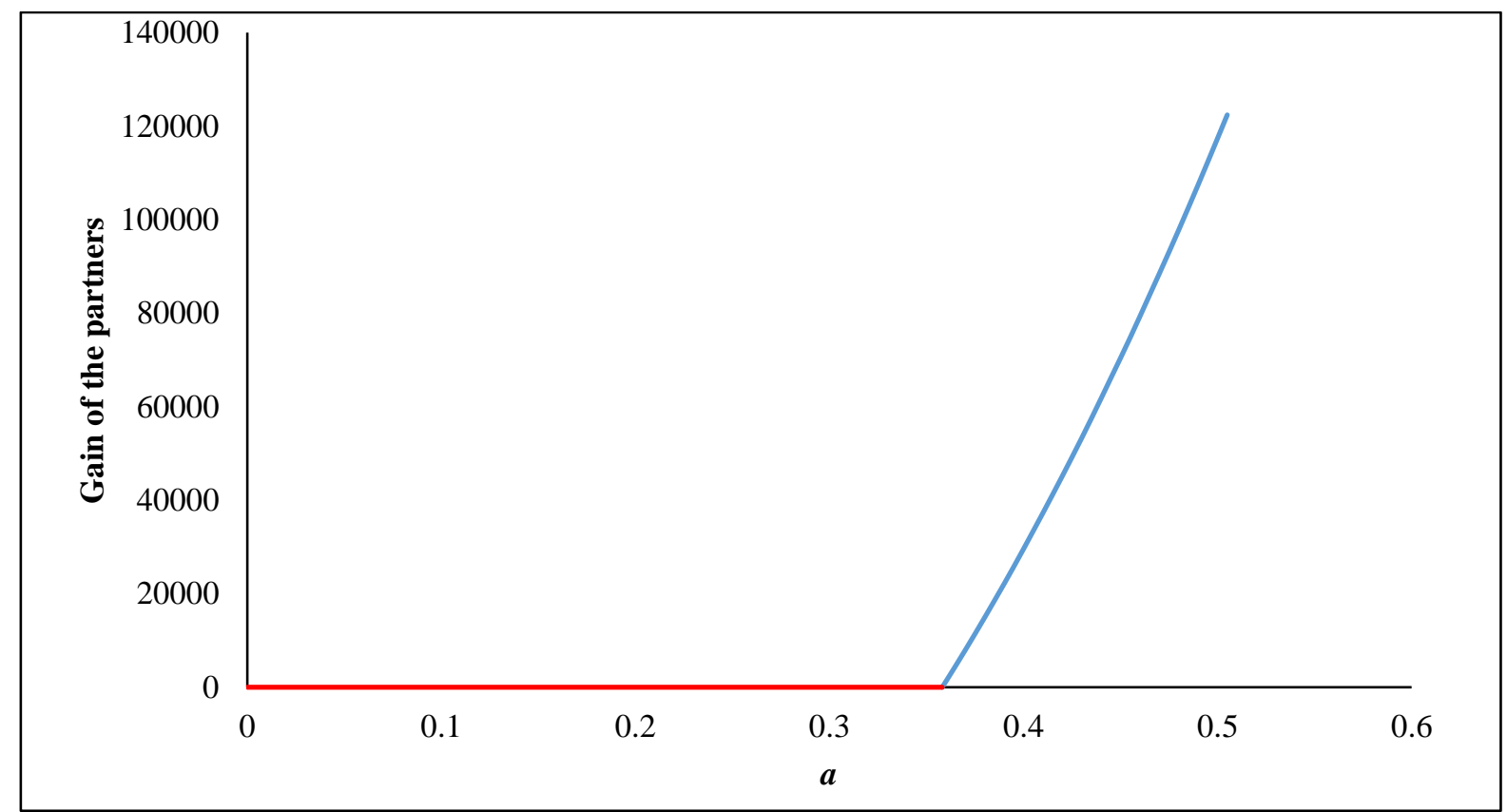

Figure 3.3 Gain of the partners $\left(\pi_{J V, F F}-\pi_{S P, F F}\right)$ vs. $a\left(R O C_{F F}=4.25\right)$ 


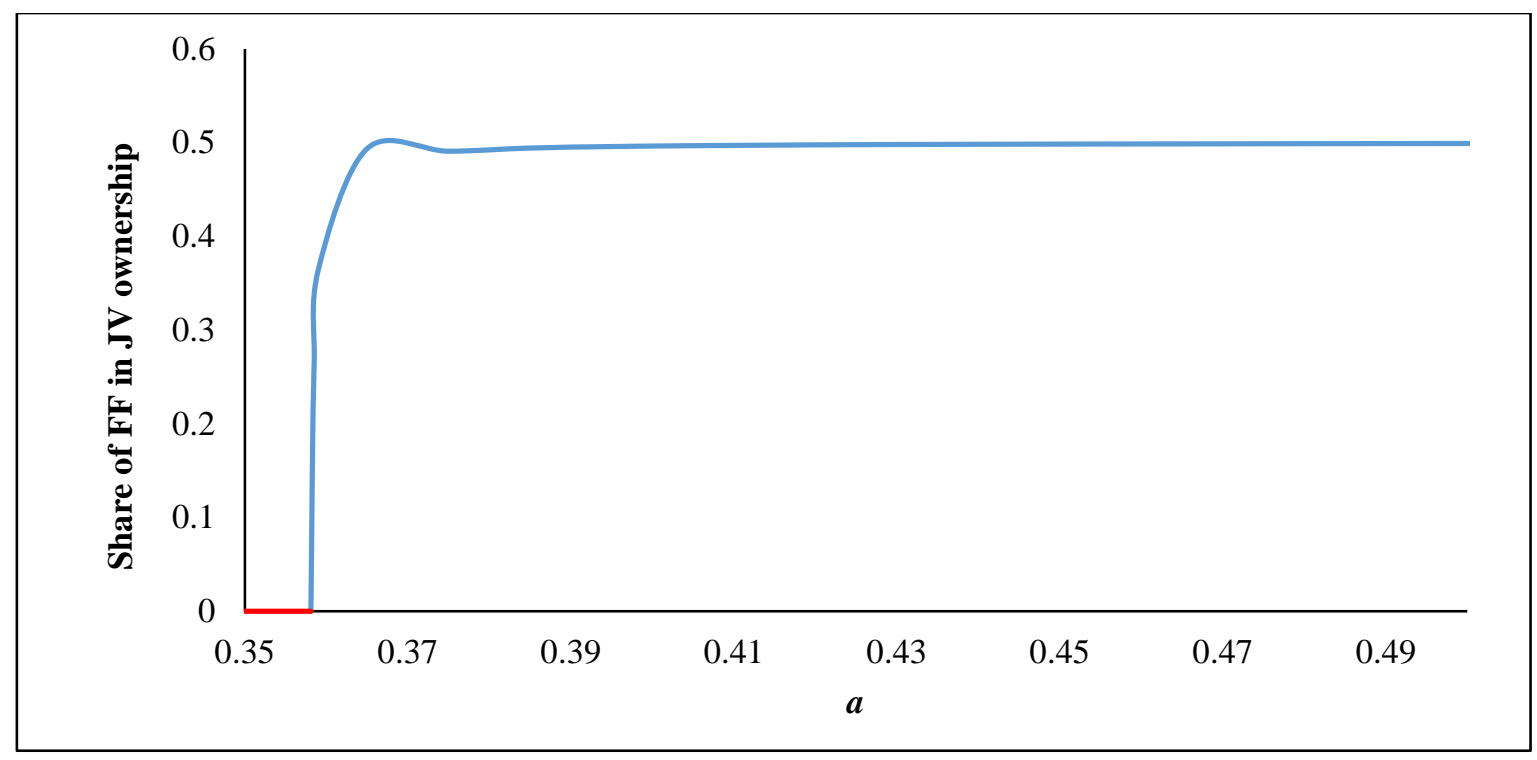

Figure 3.4 Share of the $F F$ vs. a $\left(R O C_{\mathrm{FF}}=0.34\right)$

Likewise, the derivative of the $M G_{\mathrm{S}}$ with respect to $b$ is zero. This indicates that changes in $b$ do not result in shifting the ownership structure. On the other hand, the effects of $b$ on the value of $O_{L F}$ and $O_{F F}$ are in contrast with those of $a$. In this regard, when $b$ is increased, the optimal price in Eq. 3.13 and the demand are decreased. As a result, the sales income is decreased as well. Consequently, $O_{L F}$ might be decreased until it falls below zero. Moreover, we know from Lemma 3.1 that when $O_{L F}$ is negative, the formation of $J V$ is not economically feasible. For the regarded case study in Table 3.1, the effects of $b$ on the gain of partners are shown in Figure 3.5. In this figure, the red line highlights the value of $b$ at which the formation of $J V$ is not affordable.

Figure 3.5 points out the fact that increases in $b$ lead to reductions in the gain of the partners. To some extent, the formation of a $J V$ is not justified anymore. Now, to show the effect of $b$ on the share of partners in the $J V$, we consider the state where $R O C_{F F}=0.34$. In this case, $M G_{S}>0$ and the share of the $F F$ versus $b$ is represented in Figure 3.6. 


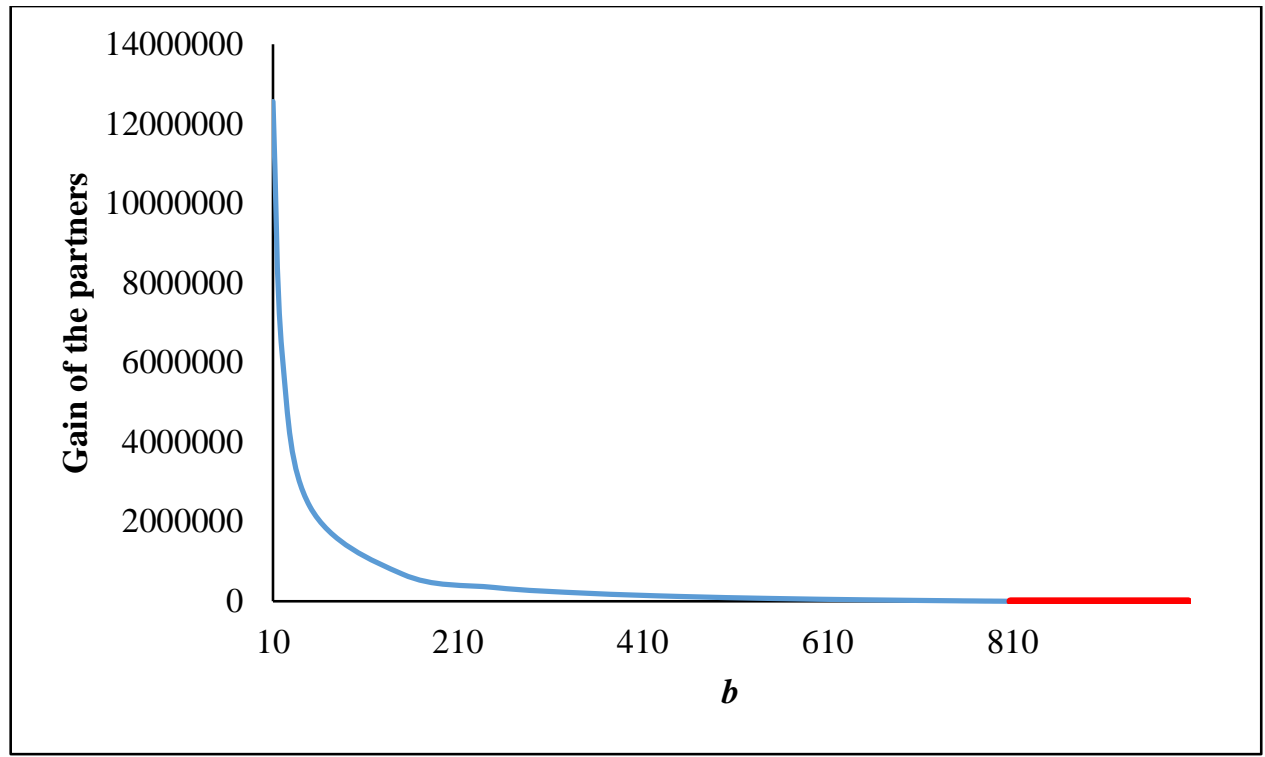

Figure 3.5 Gain of the partners $\left(\pi_{J V, F F}-\pi_{S P, F F}\right)$ vs. $b\left(R O C_{F F}=0.425\right)$

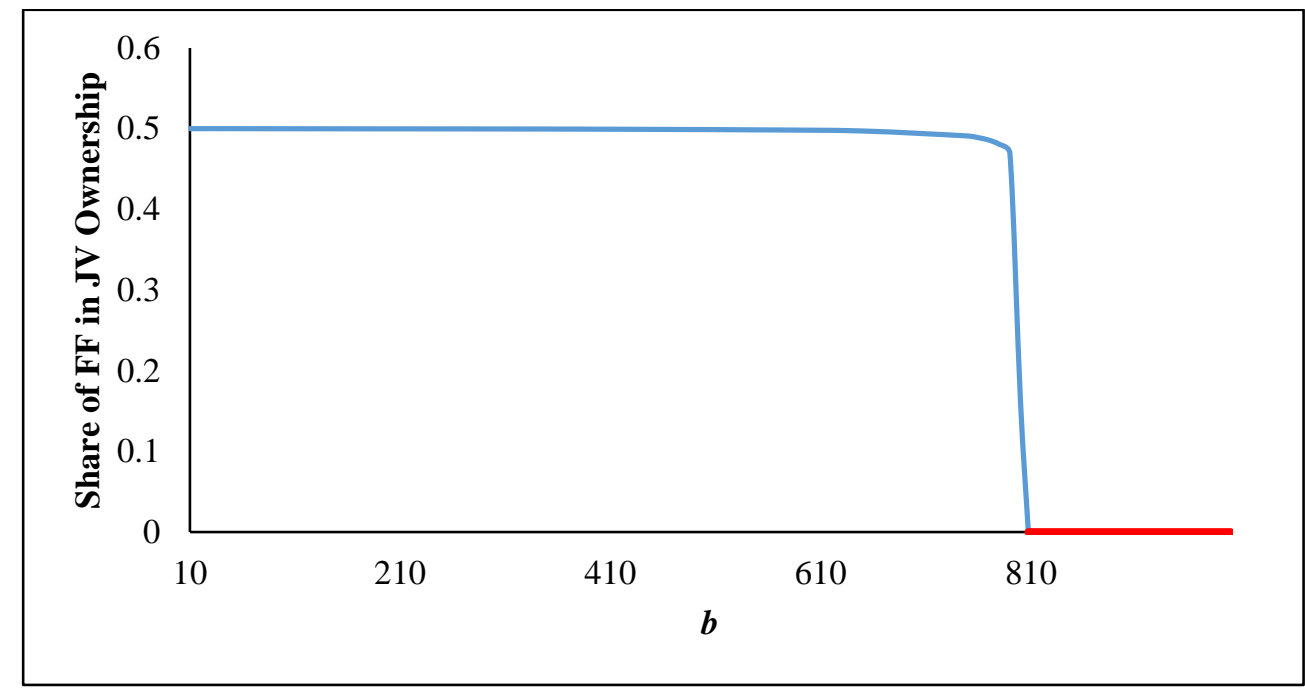

Figure 3.6 Share of the $F F$ vs. $\boldsymbol{b}\left(R O C_{F F}=0.34\right)$

Based on Figure 3.6, by increasing $b$, the share of the $F F$ on the ownership of the $J V$ will be decreased which could be explained by the negative derivative of $\varphi^{*}$ with respect to $b$ in the mentioned case study. 


\subsubsection{Changes in the fixed investment cost $(F)$}

In the proposed model, $F$ was introduced as the parameter which represents the required investment cost for establishing a $J V$. If the investment cost was very high, it might affect the decision about the formation of a $J V$. However, the sign of the $M G_{S}$ in Eq.3.26 is determined by the sign of the term $\left[\frac{R O C_{L F}}{1-\tau_{L F}}-\frac{R O C_{F F}}{1-\tau_{F F, 2}}\right]$. Hereafter, changes in $F$ will not alter the $M G_{S}$ sign and, consequently, the structure of ownership. Nonetheless, when $F$ is increased, both $O_{L F}$ and $O_{F F}$ are decreased, and when $O_{L F}$ becomes negative, the formation of a $J V$ is disregarded. As a result, by changes in $F$, one shift will occur from the affordability of a $J V$ to the state where a $J V$ is not profitable anymore. The gain of partners for different values of $F$ is represented in Figure 3.7. Again, the red line in Figure 3.7 shows the value of $F$ for which the formation of a $J V$ is not profitable.

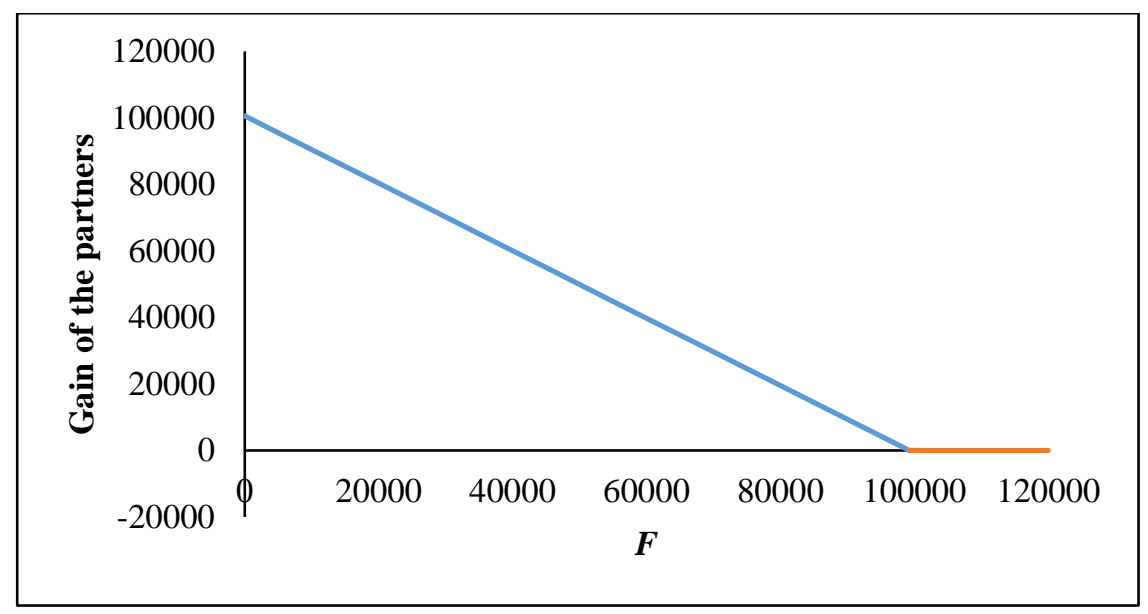

Figure 3.7 Gain of the partners vs. $F\left(R O C_{F F}=0.425\right)$

Figure 3.7 shows that when the investment cost is increased, the gain of the partner is decreased as we expected. The linear relation between gain and $F$ could also be explained in two respects. First, we show that the ownership structure (Licensee ownership in the original case study) does not alter by changing $F$. Second, in the Licensee ownership structure, the gain of the $F F$ is a linear function of the license income, this income itself is a linear function of $O_{L F}$ based on Eq. 3.24 and $O_{L F}$ is a linear function of $F$, according to Eq. 3.18. Second, to show the effect of changes in $F$ on the share of partners, we attend to the state where $R O C_{F F}=0.34$, which results in a positive $M G_{S}$ and a shared ownership structure. Figure 3.8 shows the share of the $F F$ for different values of $F$ in the considered case study. 


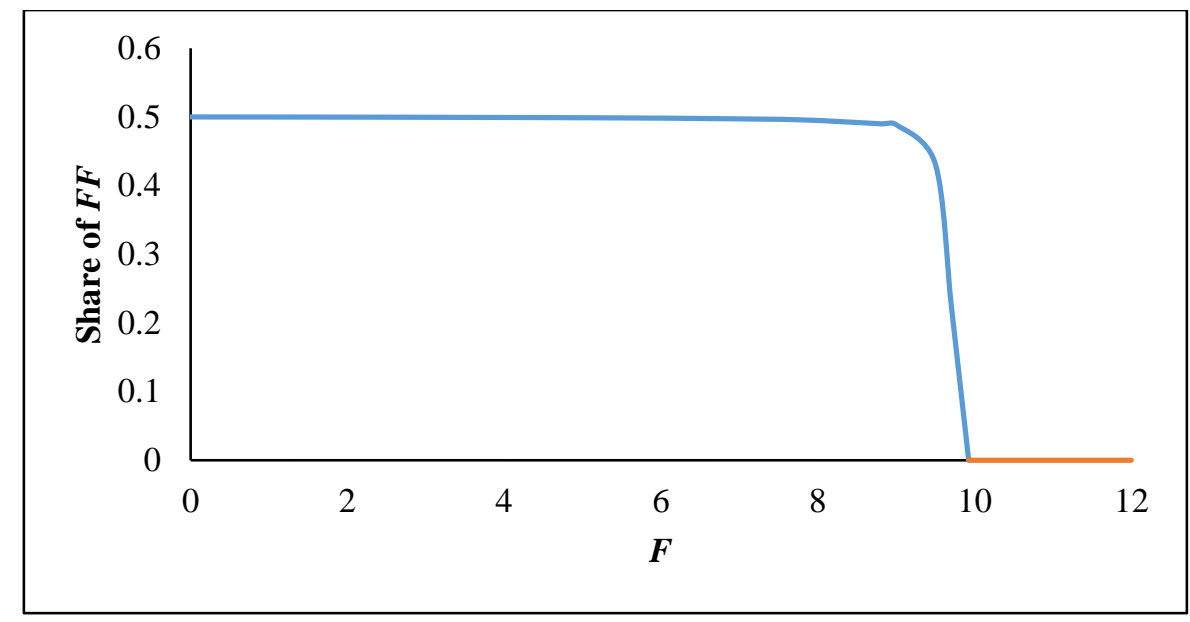

Figure 3.8 Share of the $F F$ vs. $F\left(R O C_{F F}=0.34\right)$

According to Figure 3.8, when $F$ is increased, the share of the $F F$ is decreased because in the present case study, the derivative of $\varphi^{*}$ with respect to $F$ is negative and the negativity of the derivative explains such behavior.

\subsection{Conclusion}

In this chapter, the proportional contract was considered as a base mechanism for the profit allocation problem in joint ventures. The proportional contract has been based on the twoplayers games wherein players agree on a cooperative strategy and decide to split the resulting surplus of this strategy. Further, the equal distribution of surplus is investigated as the solution of the game as the "proportional contract" which is based on this concept (Sercu, 2009; Adegbesan \& Higgins, 2011). Unlike previous studies, this chapter addresses joint venture and strategic alliance settings in terms of practical game theoretical models. All the models of the chapter were developed by considering different parameters in the real-world problem, which could affect the profitability of the firms in a $J V$. We utilized $R O C$ rates in the financial theories to formulate the stand-alone profit of the partners in the proposed contract. The appropriateness of this rate was explained by the comprehensiveness of the rate in the financial statements, comparability of the firms by this rate, and acceptability of the rate in the financial and valuation contexts. It should be noted that the stand-alone profit is a base parameter of some cooperative games, but to the best of our knowledge, its formulation has been less discussed in the context of strategic alliance and especially in a $J V$. 
The results of the proposed models revealed some facts about the strategic and operational decisions related to an alliance. It was shown that when the proportional contract mechanism is used for the profit allocation problem, the optimal operational and allocation decisions should be such that the profit of the $J V$ will be maximized. Therefore, the terms of license payment in the $J V$ agreement are tools that justify and regulate the payment planning between the firms. We discussed that the licensor partner could not change, and it is not optimal to change the pricing decisions to get more profit related to the received royalty fee. Furthermore, the marginal free-tax operational gain on the share $\left(M G_{S}\right)$ was introduced as a concept that relates the optimal ownership structure of a JV with the partners' performance in generating gain, and free of tax consideration. In this regard, we interpreted the positive values of the $M G_{S}$ for the states wherein the performance of the $F F$ in the $J V^{\prime}$ s ownership is better than that of the $L F$. So, in these states, the maximum share of the $F F$ in the ownership of the $J V$, which leads to $L P=0$, was shown to be optimal. We referred to these states as shared ownership structures. On the other hand, the negativity of the $M G_{\mathrm{S}}$ indicated the better performance of the $L F$ in generating the surplus or gain of the $J V$. Therefore, when the $M G_{S}$ is negative, it was proved that the ownership of the $L F$ is optimal, and the profit of the $F F$ should be in the form of license income. The latter case was referred to as the Licensee ownership structure in the thesis.

The decision about the formation of a $J V$ was investigated through the definition of the ideal gain of partners' concept. In this context, it was shown that the necessary and sufficient condition for the affordability of a $J V$ is the positivity of the $L F$ ideal gain. When the ideal gain of the $L F$ is negative, no gain and surplus will be generated by the cooperation of the firms, and therefore there is no strong evidence for the formation of a $J V$.

Our proposed framework and models were implemented in a case study about the production of vitamin A as a pharmaceutical component. The results of this implementation confirmed that the optimal share, royalty rate, and lump sum amount of the proposed model were not far from the ones that are implemented in real-world problems. Also, we showed how different restrictions on the real-world conditions, such as the maximum allowable ownership of foreign firms in the local market or maximum ability of partners in providing the required investment of a $J V$, could be addressed by the proposed model.

Finally, we conducted an in-depth sensitivity analysis and showed what effects different values of the parameters have on the ownership structure and the decision about the formation of a $J V$. The first parameter considered in this analysis was $R O C_{F F}$. The chapter results indicated 
that changes in this parameter affect both the shares of the partner and the ownership structure. Moreover, when the local partner has alternatives to choose from among some foreign licensor, the best choice for the $L F$ is to select the one that has $R O C_{F F} \geq R O C_{F F}^{B P}$. Doing so, the $L F$ obtains full ownership of the $J V$, and the profit of the $J V$ is maximized. Other parameters that could be changed and considered through the sensitivity analysis were the market scale parameter $(a)$, the sensitivity of demand on the price parameter $(b)$, and the estimated investment cost of the $J V$, i.e., $F$. The effects of changes in these parameters are explained mathematically and by doing the sensitivity analysis on the considered case study. 


\section{Chapter 4: A new model of the license valuation of pharmaceutical products in an oligopoly market structure}

In the previous chapter, setting a license contract between a local firm as licensee and a foreign firm as licensor was discussed regarding simple assumptions. In fact, the main aim of previous models was to determine which ownership structure is optimal by taking into account the capabilities of the partners. However, the valuing environment of firms and alliances is usually more complex than the models of the previous section. More complex market structure, different sources of uncertainty, multi-period nature of valuation method, as well as the need for considering other decisions such as capacity problem are issues that entail more sophisticated models as discussed in this chapter.

Technology protection through license contracts is widespread when technology owners decide to grant production permissions to other manufactures. This strategy provides many advantages for both the licensor and the licensee, such as market access, reduction in the transaction cost, reduction in the production cost, etc. The license valuation is essential for granting the license and determining the share of partners in the generation of participation synergy. This problem becomes more complicated when the licensor and licensee are from different countries. Unlike other researchers, this chapter develops a novel license valuation mechanism which considers the optimal capacity planning decisions by considering the transaction cost and market capabilities as two important determinants of the license value. Further, this chapter also focuses on the optimal financial resources by modeling the demand uncertainty through real life-based scenario generation and addressing the pricing decisions for the licensed products in a market that usually has an oligopoly structure. Therefore, the proposed model addresses the practical issues of market capabilities, transaction cost, capacity planning, financing, and demand uncertainty while developing a new license valuation model for pharmaceutical products and a backward dynamic programming method is also proposed for solving the model. The proposed model is applied for valuing the license of a real pharmaceutical product, and an in-depth sensitivity analysis is carried out to validate the model in a real pharmaceutical company.

\subsection{Introduction}

The advantages of cooperative alliances were mentioned in the previous chapters. However, the value of resources plays the main role in accounting for shareholders' equity and the 
financial standing of firms. In this regard, the resources could be categorized into tangible and intangible resources. Tangible resources are physical items, including cash, inventory, machinery, land, or buildings. These items can be easily liquidated and have a set value in the firms' financial statements. On the other hand, intangible resources stand for nonphysical assets or Intellectual Property $(I P)$. It is challenging to assign intangible resources explicit value because their future outcome is uncertain and hard to predict. A license contract has been mostly designated to account for or protect the right of the intangible assets' owner in various forms, including some shares in the ownership, royalty fee, lump sum, or a combination of these forms (Sercu, 2009).

It is notable that traditional license valuation methods have some shortcomings for evaluating the patients or technologies in an international alliance formed by the cooperation of firms from different countries. Among these shortcomings, ignoring the other partners' efforts, the inability to distinguish the value of different $I P \mathrm{~s}$, and disagreement on the goodwill of some $I P \mathrm{~s}$ by partners from different countries could be referred. Covering the mentioned shortcomings, the model of this chapter makes the following contribution to the literature:

1) First, the proposed model is a multi-period model and considers the resulting cash flow of licensed products on a long-term horizon. Dynamic and long-term considerations are the main concept in the asset valuation models, which introduces the value as the net present value of the generated cash flows over time received by the seller/vendor of a product (Schwartz, 2004).

2) Besides the patent or production technology, the licensee might even claim some profits due to its role in the reduction of transaction costs and its market share. In the proposed valuation model, the transaction cost is added to the production cost, and the market share is considered using the concept of conjecture price, which reflects the power of producers in the market.

3) The market of technology and patented products in the case of no control over price is mainly an oligopoly. This is due to the nature of the technology owned by a few manufacturers. To model the market structure of an oligopoly, the conjecture price approach is utilized in the models of this chapter. In this mechanism, the equilibrium price of the market is determined by the conjecture which each supplier estimates about the change of the equilibrium price against the change in the production of each supplier (de Haro et al., 2007; Moiseeva et al., 2017). 
4) It is clear that the demand for the market in the future is uncertain. Moreover, according to the economic and system dynamics theories, path dependency and market shock might affect future demands (Sterman, 2000). These assumptions are prevalent in the option-based valuation method, which considers the possibility of a path-dependent relationship and jump-diffusion process in the generated scenarios or paths of the uncertain demand (Longstaff \& Schwartz, 2001). In the proposed model, the stochastic programming method is utilized to model uncertainty in demand, and we explain how the stochastic scenarios are generated to address these assumptions in the model.

5) Once starting a new firm subject to the availability of a production license is intended, finance sourcing finds its importance (as for any other business plans) (Jing \& Seidmann, 2014; Jensen et al., 2018). To determine the required financial resources and plan finance sourcing, a proper estimation of all incurred costs is necessary. In the proposed model, such costs, including production cost, depreciation cost, financial interest expense, and transaction cost, are considered. We present the relationships of such cost items in the form of mathematical programming constraints.

6) The mathematical programming representation of the model enables the production capacity of the intended firm to be decided. Therefore, the proposed model specifies the value of the IP assets and determines the optimal capacity.

7) The thesis provides an innovative solution approach using the dynamic programming method and utilizing the base model in chapter 3. So, it is also explained how the general model of the chapter could be the extension of the base model. Moreover, the general model has options such as the possibility of alliance liquidation, which makes the proposed model more realistic and in line with the sophisticated valuing option method in such as Longstaff and Schwartz (2001) and Richards and Rickard (2014).

\subsection{Model Description}

Given the complexity of the model in this chapter, we first provide an overview of the model's general assumptions. Then, in the following sections, the various aspects of the problem are investigated.

The general assumptions used in the model are as follows:

1. The market structure is an oligopoly in the local country because the production entails specialized technology owned by several manufacturers. 
2. The import of the product entails various transaction costs such as customs duties, transportation, formalities and so on. Part of the transaction costs are paid by manufacturers, and the other part is the duty of local consumers.

3. The expected benefits of cooperation for manufacturers are the reduction in the transaction costs, the higher utility of customers due to the reduced price, gaining local government protection, and gaining more market share.

4. Companies are often faced with capacity development over time for reasons such as increasing their share in other markets, increasing product demand due to increased quality and $\mathrm{R} \& \mathrm{D}$ activities, etc. On the other hand, in a particular market, if the conditions are constant, the competition will usually increase over time, and companies will be faced with capacity reductions rather than capacity building (for example, in Iran, in feasibility studies of knowledge-based companies usually decrease in market share in the future is anticipated). So, we disregard any capacity expansion after the first establishment and regard such development as a new improvement and renovation project entailing future considerations.

5. An exclusive license contract is studied whereby no other licensee could sell the licensed product on the local market. Furthermore, the contract permits the licensee to sell the product just in the local market, not in any other market. On the other hand, companies owning technology or patents restrict the sale of products to a particular market and allow the alliance to sell its product in other markets for reasons such as protecting technology or making more money from patenting them to other companies. The opposite is also true. For example, due to the high establishment cost of an alliance, the technology owner company can assign its share of other markets to the new alliance (for example, a joint venture agreement between NIOC and a foreign company). However, the sales of products in other markets are not assessed in the present study because it needs a detailed evaluation of issues such as international trade, market segmentation, etc., which is suggested for future research.

6. The model addresses strategic issues, so it is a multi-period model. This provision is in accordance with the changeable demands of products during different periods of their life cycle as well as the patent terms of new technologies. For example, once an exclusive technology expires, the local manufacturer could achieve it without a 
partnership alliance and license fees. It is also assumed that the average life of the alliance is predetermined and is less than the remaining time of the patent.

Also, considering the complexity of the model, we first present the questions that we seek to answer by this model as follows:

1) How does market structure affect demand and price equilibrium of the product, and what changes can the alliance make to market structure and price equilibrium?

2) How can we predict the alliance revenue and estimate the optimal capacity of the company with which it operates in the market?

3) How should alliance revenue be divided among the partners?

The following sections present different parts of the model until the deterministic and uncertain models of the cooperative alliance are presented. Finally, the dynamic programming-based solution method of the chapter is presented.

\subsection{General model}

The following notations are regarded to describe the model of this chapter:

$\begin{array}{ll}\text { Parameter } & \text { Description } \\ I & \text { The index of active firms in the local market } \\ J & \text { The index of firms in alliance }(j=F \text { for the licensor and } j=L \text { for the licensee) } \\ L F . & \text { The index used for denoting the alliance of licensor and licensee } \\ T & \text { The index of time periods } \\ \beta & \text { The cost of developing each unit of depreciable capacity in the alliance } \\ \alpha & \text { The cost of developing each unit of non-depreciable capacity in the alliance } \\ M Y_{L, t} & \text { The maximum funding of licensee in period } t \\ M Y_{F, t} & \text { The maximum funding of licensor in period } t \\ M I_{t} & \text { The maximum available loan of the alliance in period } t \\ \gamma & \text { The multiplier of initial investment -which could be received as loans. } \\ \delta_{i, t}\left(\delta_{i, t, \Omega}\right) & \text { Per unit production cost of firm } i \text { and period } t \text { (in scenario } \Omega \text { ) } \\ t c r_{i, t}\left(t c r_{i, t, \Omega}\right) & \text { Per unit transaction cost of firm } i \text { in period } t \text { (in scenario } \Omega \text { ) } \\ \theta_{i, t}\left(\theta_{i, t, \Omega}\right) & \text { The conjecture price of firm } i \text { in period } t \text { (in scenario } \Omega \text { ) } \\ E_{t}\left(E_{t, \Omega}\right) & \text { The market size in period } t \text { (in scenario } \Omega \text { ) } \\ a_{t}\left(a_{t, \Omega}\right) & \text { Price elasticity of demand in period } t \text { (in scenario } \Omega \text { ) } \\ r d & \text { The interest rate on loan given to the alliance } \\ p(\Omega) & \text { The probability of scenario } \Omega \\ M & \text { A big number }\end{array}$




$\begin{array}{ll}\text { Variables } & \text { Description } \\ P C_{i, t}\left(P C_{i, t, \Omega}\right) & \text { The capacity of firm } i \text { in period } t \text { (in scenario } \Omega \text { ) } \\ O_{t}\left(O_{t, \Omega}\right) & \text { The binary variable equals } 1 \text { if the alliance is active in period } t \text { and } 0 \text { if it is } \\ & \text { liquidated in period } t \text { (in scenario } \Omega \text { ) } \\ S_{t}\left(S_{t, \Omega}\right) & \text { The net income of alliance before interest and taxes } \\ D P_{t}\left(D P_{t, \Omega}\right) & \text { Capacity depreciation of alliance in period } t \text { (in scenario } \Omega \text { ) } \\ B V_{t}\left(B V_{t, \Omega}\right) & \text { The book value of alliance's capacity in period } t \text { (in scenario } \Omega \text { ) } \\ S B V_{t}\left(S B V_{t, \Omega}\right) & \text { The residual value of the alliance's capacity if it is liquidated in period } t \text { (in } \\ r_{f} & \text { scenario } \Omega \text { ) } \\ p_{t}\left(p_{t, \Omega}\right) & \text { The royalty fee in each period received by the licensor } \\ q_{i, t}\left(q_{i, t, \Omega}\right) & \text { Production quantity of firm } i \text { in period } t \text { (in scenario } \Omega \text { ) } \\ I_{t}\left(I_{t, \Omega}\right) & \text { The remaining loan of the alliance in period } t \text { (in scenario } \Omega \text { ) } \\ S I_{i, t}\left(S I_{i, t, \Omega}\right) & \text { The remaining loan of the alliance in period t if it is liquidated (in scenario } \Omega \text { ) } \\ T I_{F, t}\left(T I_{F, t, \Omega}\right) & \text { The taxable dividend income of licensor in period } t \text { (in scenario } \Omega \text { ) } \\ T I_{L, t}\left(T I_{L, t, \Omega}\right) & \text { The taxable dividend income of licensee in period } t \text { (in scenario } \Omega \text { ) } \\ A L_{F, t}\left(A L_{F, t, \Omega}\right) & \text { The cumulative loss of licensor at the end of period } t \text { (in scenario } \Omega \text { ) } \\ A L_{L, t}\left(A L_{L, t, \Omega}\right) & \text { The cumulative loss of licensee at the end of period } t \text { (in scenario } \Omega \text { ) } \\ T X_{F, t}\left(T X_{F, t, \Omega}\right) & \text { The net taxable dividend income of licensor in period } t \text { (in scenario } \Omega \text { ) } \\ T X_{L, t}\left(T X_{L, t, \Omega}\right) & \text { The net taxable dividend income of licensee in period } t \text { (in scenario } \Omega \text { ) } \\ L P_{F, t}\left(L P_{F, t, \Omega}\right) & \text { The license income of licensor in period } t \text { (in scenario } \Omega \text { ) } \\ N C_{j, t}\left(N C_{j, t, \Omega}\right) & \text { The net cash flow of firm } j(j \in\{L, F\}) \text { in period } t \text { (in scenario } \Omega \text { ) }\end{array}$

Other sub-sections describe parts of the model as the following:

\subsubsection{Depreciation}

In accordance with accounting standards, every asset has a useful life span, and during its life, the value of the asset is diminished at any period. This refers to the depreciation cost, which leads to the loss in the value of assets. Depreciation is a tax-deductible expense that affects the profitability of firms. An asset is no longer capable of being used in production after its expiry. Another point is that in the cash flow-based valuing methods, sometimes the continuation of a business is not affordable, so its termination is preferable. In the proposed model, the decision about the liquidation of an alliance is prepared in line with the valuing models. Although the reduction of assets and selling them might be a possibility in some business contexts, in the present study, we only consider capacity reduction if the alliance is liquated. 
In the present study, we use the straight-line method to calculate the depreciation of capacity. It is assumed that acquisition of a capacity for production of $x$ units per year costs $\beta . x$ and the useful life of the capacity is $n$. In the straight-line method, it is assumed that the given capacity (or asset) will generate a value of $V$ throughout its lifetime each year. Hence, the value of the asset over $n$ years is $n . V$.

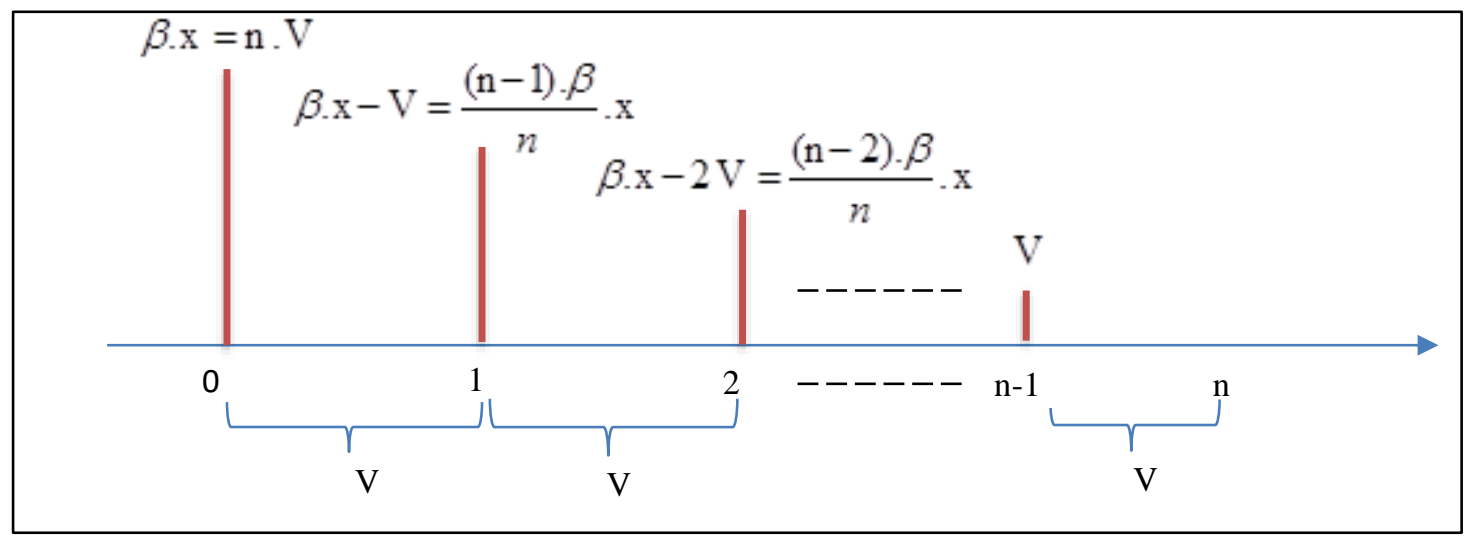

Figure 4.1 Asset value over time in the straight-line depreciation method

According to Figure 4.1, the book value of capacity will be equal to $(n-1) . \beta / n$ in period 1 and $(n-\tau) \cdot \beta / n$ in period $\tau$.

However, the capacity can be divided into two parts, depreciable and non-depreciable. Facilities like a land that have an infinite lifetime are not subject to depreciation and their book value at any time usually is equal to their primary purchasing cost. However, the book value of the depreciable capacity is also calculated according to Figure 4.1. The purchasing costs of depreciable and non-depreciable capacity for the production of $x$ units are set equal to $\beta . x$ and $\alpha . x$ respectively.

To determine the equilibrium price, the capacity level and capacity generation expenses should be evaluated for all suppliers in the local market which are characterized by index $i$. The capacity of other suppliers except the new firm is considered an uncertain parameter. However, for the new firm, if it is assumed that the selling price of the capacity at the time of liquidation is equal to its book value and takes into account the above descriptions and notations, then the depreciation evaluation constraints will be as below:

\section{The Depreciation evaluation constraints set $(T)$}

$$
\begin{aligned}
& D P_{0}=S B V_{0}=0 \\
& B V_{0}=\beta_{i} \cdot P C_{L F, 0}
\end{aligned}
$$




$$
\begin{array}{ll}
D P_{t}=\frac{B V_{t-1}}{n-t+1} & \forall t \in T, t \geq 1 \\
B V_{t}=B V_{t-1}-D P_{t} & \forall t \in T, t \geq 1 \\
B V_{t-1}+\alpha \cdot P C_{L F, 0}-M \cdot\left(1-O_{t-1}\right. & \left.+O_{t}\right) \leq S B V_{t} \leq B V_{t-1}+\alpha \cdot P C_{L F, 0} \quad \forall t \in T, t \geq 1 \\
S B V_{t} \leq M .\left(O_{t-1}-O_{t}\right) & \forall t \in T, t \geq 1 \\
D P_{t}, B V_{t}, S B V_{t}, P C_{i, t} \geq 0 & \forall i, t \in T, t \geq 1 \\
O_{t} \in\{0,1\} & \forall t \in T
\end{array}
$$

Eq. (4.1) sets the depreciation at the beginning of the alliance and the variable $S B V_{0}$ equal to zero. Eq. (4.2) determines the book value of the depreciable capacity at time zero. According to Eq. (4.3), the depreciation of capacity in other periods is equal to the book value of the capacity in the preceding period divided by its remaining useful life. The depreciation expense is reduced from the book value of a period to determine the book value in the next period, as shown in Eq. (4.4). In Eqs. (4.5) and (4.6), the book value of capacity in the case of the alliance liquidation is calculated. Accordingly, if the company is not liquidated, the residual value in that period is not taken into account in the cash flow of the alliance. Eqs. (4.7) and (4.8) also describe the decision variables.

Next, to relate the capacity of each supplier to its funding (Stakeholders equity or the external loan) and its decision about liquidation, the constraint set as below is regarded:

\section{The capacity generation constraint sets $(T)$}

$$
\begin{array}{ll}
\left(\beta_{i}+\alpha_{i}\right) \cdot P C_{L F, 0}=N C_{L, 0}+N C_{F, 0}+I_{0} & \\
I_{L F, 0}=\gamma \cdot\left(N C_{L, 0}+N C_{F, 0}\right) & \\
N C_{F, 0}=\varphi\left(N C_{F, 0}+N C_{L, 0}\right) & \\
N C_{j, 0} \leq M Y_{j} & j \in\{L, F\} \\
P C_{L F, t-1}-M .\left(1-O_{t}\right) \leq P C_{L F, t} \leq P C_{L F, t-1} & \forall t \in T \\
P C_{L F, t} \leq M . \mathrm{O}_{t} & \forall t \in T \\
O_{t} \leq O_{t-1} & \forall t \in T, t \geq 1 \\
I_{t}, N C_{L, t}, N C_{F, t} \geq 0 & \forall t \in T
\end{array}
$$

Eq. (4.9) shows that the costs of providing the required capacity in the initial period are prepared using the firms' equity and the amount of loan received by the firms. Eq. (4.10) relates to the received loan of the alliance to its initial investment. This assumption is in accordance with the case study of the chapter. Eq. (4.11) states that the initial investments of the licensor and licensee are proportional to their share in the ownership of the alliance. Eq. (4.12) restricts the initial investments of the licensor and licensee to their maximum possible funding. Eqs. 
(4.13) and (4.14) state that if the alliance is not liquidated in the period $t\left(O_{t}=1\right)$, then its capacity in that period is similar to the previous period. However, in the case of liquidation, the capacity is reduced to zero. Eq. (4.15) illustrates that if the alliance is liquidated in a period, it will be inactive in the proceeding periods too. Eq. (4.16) also interprets the used variables in the equations.

It is also notable that the beginning period in the model corresponds to the completion of the construction phase. So, all investment costs at period zero are actually the discounted costs of the construction phase.

\subsubsection{Loan repayment constraints}

As mentioned, firms could prepare a part of their capacity development cost through the loan, which depends on their stakeholders' initial equity. Loans should be repaid after the predetermined time $m_{0}$. Moreover, it is prescribed that if the liquidation occurs, the remaining principal of the loan should be repaid immediately. Hence, assuming that the principal of the loan at the end of the period $t$ is $I_{t}$, the remaining amount of the loan if the company is liquidated in the period $\mathrm{t}$ is $S I_{t}$.Hence, the following constraints hold for the loan repayment process:

\section{The loan evaluation constraints set $(T)$}

$$
\begin{array}{ll}
I_{t}=I_{t-1}-V_{t} & \forall t \in T, t \geq 1 \\
V_{t}=\frac{I_{0}}{m} & m_{0} \leq t<m_{0}+m \\
V_{t}=0 & t<m_{0} \text { or } t \geq m_{0}+m \\
S I_{t} \leq M .\left(O_{t-1}-O_{t}\right) & m_{0} \leq t<m_{0}+m \\
I_{t}-M .\left(1-O_{t-1}+O_{t}\right) \leq S I_{t} \leq I_{t} & m_{0} \leq t<m_{0}+m \\
S I_{t}=0 & t<m_{0} \text { or } t \geq m_{0}+m
\end{array}
$$

Eq. (4.17) indicates that the remaining principal of the loan at the end of each period is equal to the remaining principal of the loan in the previous period minus the amount of loan repayment in the current period. Eq. (4.18) divides the repayment of the loan equally in the repayment period. Eq. (4.19) sets the repayment amount of the loan out of the repayment period equal to zero. Based on Eqs. (4.20), (4.21), and (4.22), the remaining principal of the loan in the case of liquidation in the repayment period should be settled immediately, and the related variable in the other period equals zero. 


\subsubsection{Local market structure considering transaction costs}

Transaction costs are expenses incurred when buying a product or delivering a service. These include contract costs, shipping costs, customs duties, ordering costs, and all costs that change the ownership of a product from sellers to buyers.

In this research, for the sake of simplicity and by many real-world problems, it is assumed that there is a linear relationship between transaction costs and the quantity supplied to the market. Hence, denoting the proposed price of the supplier to the buyer by $p_{i}$, the transaction cost of the customers per unit of the product supplied by firm i by $\operatorname{trc} c_{i}^{c}$, and the equilibrium price of the market for the homogenous product by $p$, then Eq. (4.23) is valid:

$$
p=p_{i}+\operatorname{trc} c_{i}^{c} \rightarrow p_{i}=p-\operatorname{trc} c
$$

On the other hand, if the affordable part of the transaction cost for the supplier $i$ per each unit of the product is $\operatorname{trc}_{i}^{s}$, then the firm $i$ 's income $\left(\pi_{i}\right)$ will be as Eq. (4.24):

$$
\begin{aligned}
& \pi_{i}=\left(\left(p-t r c_{i}^{c}\right) \cdot q_{i}-\left(C_{i}\left(q_{i}\right)+t r c_{i}^{s} \cdot q_{i}\right)\right)=p \cdot q_{i}-\left(C_{i}\left(q_{i}\right)+\left(t c r_{i}^{s}+t c r_{i}^{c}\right) \cdot q_{i}\right)= \\
& p \cdot q_{i}-C_{i}^{a}\left(q_{i}\right)
\end{aligned}
$$

In Eq. (4.24), $C_{i}\left(q_{i}\right)$ is the production cost incurred by the producer $i$, and we assume that it is a linear function of the production quantity. Hence, if the unit cost of production for firm $i$ is shown by $\delta_{i}$, then the relation $C_{i}\left(q_{i}\right)=\delta_{i} \cdot q_{i}$ is valid. It is clear that $C_{i}\left(q_{i}\right)$ is a convex, continuous, and derivative function. Moreover, if the total transaction cost is defined as $t c r_{i}=t c r_{i}^{c}+t c r_{i}^{s}$, then the adjusted cost function $C_{i}^{a}\left(q_{i}\right)$ is introduced as $C_{i}^{a}\left(q_{i}\right)=C_{i}\left(q_{i}\right)+\left(t c r_{i}\right) \cdot q_{i} . C_{i}^{a}\left(q_{i}\right)$ which is a convex and continuous function. Therefore, the uniqueness of the market equilibrium price can be proved by this definition (Barquin et al., 2004).

In oligopoly, different approaches have been proposed to calculate the equilibrium prices, including the conjectural equilibrium (CE) methods (Ventosa et al., 2005), Nash-Cournot equilibrium (Nash, 1950), supply function equilibrium (SFE) (Baldick et al., 2004) and conjectural supply function equilibrium (CSFE) (Day et al., 2002). In the present study, we use the $\mathrm{CE}$ to model the equilibrium price of oligopoly for homogeneous products as this approach can be interpreted and developed into problems with technical constraints. In this approach, like the Nash-Cournot equilibrium approach, manufacturers compete on the number of products offered to the market and also conjecture the strategies or responses of other 
manufacturers towards the market. Various studies have proposed different models to formulate the conjectured response. In the review article by Diaz et al. (2010), the types of conjectured responses and their equivalency as well as how to measure them are described. In the present study, we consider the conjectural price response index as a conjecture of company $i$, which reflects the effect of company $i$ 's production on market price. By definition, $\theta_{i}$ is a conjectured partial derivative, which is the belief of a company that its production changes as the price changes, and we call it a conjectured price response (Diaz et al., 2010). In fact, each manufacturer $i$ assumes that there is a definite change in price when it changes its production rate and we call it the $\theta_{i}$ conjectured price response, which is calculated by the equation (4-25).

This is the conjecture of firms about the changes in the equilibrium price due to the increase in the production quantity of firm $i$ by one unit (Diaz et al., 2010).

$$
\theta_{i}=-\frac{\partial p}{\partial q_{i}}
$$

Total market demand $d$ is also modeled as $d=E-\alpha \cdot p$ which is a linear function of the equilibrium price. Here, $E$ is the market size and $\alpha$ is the price sensitivity coefficient in the demand function. In this case, the maximum market price is $E / \alpha$, and when the minimum cost of supplying one unit of a product by a supplier is greater than the maximum market price, the supplier has no incentive to enter the market. Clearly, Lemma 4.1 could be stated for associating the transaction cost and the aim of firms for entering markets:

Lemma 4.1: If $t c r_{i}>\frac{E}{\alpha}-\delta_{i}$, then the firm $i$ has no incentive to enter the market.

Lemma 4.1 introduces the transaction cost as a cause of why some firms are active in some markets. For example, in the case of economic and political sanctions, long distances, foreign trade restriction laws, and high tariffs, the transaction cost tends to a great number and satisfies Lemma 4.1.

Regardless of technical constraints such as capacity, Eq. (4.26), which represents the first-order condition of Eq. (4.24), holds in the equilibrium price (Diaz et al., 2010):

$$
\frac{\partial \pi_{i}}{\partial q_{i}}=p+q_{i} \cdot \frac{\partial p}{\partial q_{i}}-\frac{\partial C_{i}^{a}\left(q_{i}\right)}{\partial q_{i}}=p-q_{i} \cdot \theta_{i}-\frac{\partial C_{i}^{a}\left(q_{i}\right)}{\partial q_{i}}=0 \forall i
$$


Given the convexity of $C_{i}^{a}\left(q_{i}\right)$ for all firms and their capacity constraints as the most important technical constraint, it is proven that the minimization problem as Eq. (4.27) is equivalent to the first-order condition of Eq. (4.26) (Barquin et al., 2004):

$$
\begin{array}{ll}
\min _{\substack{q_{i}, d \\
\text { s.t. }}} & \sum_{i} \hat{C}_{i}\left(q_{i}\right)-v(d) \\
& \sum_{i} q_{i}=E_{t}-\alpha_{t} \cdot p_{t}: \\
& 0 \leq q_{i} \leq P C_{i} \quad \forall i
\end{array}
$$

In the above equation, $\hat{C}_{i}\left(q_{i}\right)=C_{i}^{a}\left(q_{i}\right)+q_{i}^{2} \cdot \theta_{i} / 2$ is the Expanded Cost Function, $v(d)=\int_{0}^{d} p(d) \mathrm{d} d=(1 / \alpha) .\left(E d-d^{2} / 2\right)$ is demand utility, and $P C_{i}$ is the production capacity of firm $i$.

By adding the index $t$ for denoting the market equilibrium in period $t$, the following model should be considered for determining the equilibrium price in each period:

\section{The equilibrium price model $(t)$}

$$
\begin{gathered}
\min \sum_{i}\left(\left(\delta_{i, t}+t c r^{i, t}\right) \cdot q_{i, t}+q_{i, t}^{2} \cdot \theta_{i, t} / 2\right)-\frac{1}{\alpha_{t}}\left(E_{t} \cdot\left(E_{t}-\alpha_{t} \cdot p_{t}\right)-\left(E_{t}-\alpha_{t} \cdot p_{t}\right)^{2} / 2\right) \\
\text { s.t } \quad \forall t \\
\sum_{i} q_{i, t}=E_{t}-\alpha_{t} \cdot p_{t} \quad \forall i, t: \lambda_{i}(t), \mu_{i}(t) \\
0 \leq q_{i, t} \leq P C_{i, t}
\end{gathered}
$$

It should be noted that before entering the market, companies must make strategic decisions such as the capacity of the period so that model (4-28) can determine the equilibrium price. Hence, in order to optimize their capacity, which will be described later in the partners' integrated revenue model, companies choose the market equilibrium (4-28) as a low-level model in their strategic capacity determination model. On the other hand, as it will be explained, JV, after its formation, is introduced as a market leader and determines its production rate. Therefore, the value of $q_{J V, t}$ at the second level, i.e. model (4-28), is set as a parameter and the constraint $0 \leq q_{J V, t} \leq P C_{J V, t}$ is passed to the first level. Keeping this explanation in mind and assuming the convexity of cost functions, we use the K.K.T conditions instead of the second-level model (4-28), which leads to the optimal solution of the model (428). The K.K.T conditions are equivalent to the model (4-28) in the form of equation (4-29): 
The K.K.T conditions of the equilibrium price model $(t)$

$$
\begin{array}{lr}
\left(\delta_{i, t}+t c r^{i, t}\right)+q_{i, t} \cdot \theta_{i, t}+E_{t} \cdot \frac{\partial p_{t}}{\partial q_{i, t}}-\frac{\partial p_{t}}{\partial q_{i, t}} \cdot\left(E_{t}-\alpha_{t} \cdot p_{t}\right)-\lambda_{i, t}+\mu_{i, t}=0 \Rightarrow & \\
\left(\delta_{i, t}+t c r^{i, t}\right)+q_{i, t} \cdot \theta_{i, t}-E_{t} \cdot \theta_{i}+\theta_{i} \cdot\left(E_{t}-\alpha_{t} \cdot p_{t}\right)-\lambda_{i, t}+\mu_{i, t}=0 & \forall i \\
0 \leq q_{i, t} \leq P C_{i, t} & \forall i \\
\lambda_{i, t} \cdot\left(q_{i, t}-P C_{i, t}\right)=0 & \forall i \\
\mu_{i, t} \cdot q_{i, t}=0 & \forall i \\
d_{t}=\sum_{i} q_{i, t}=E_{t}-\alpha_{t} \cdot p_{t} &
\end{array}
$$

In (4.49), $\lambda_{i, t}$ and $\mu_{i, t}$ are the Lagrangian multipliers of the constraints $q_{i, t} \leq P C_{i, t}$ and $q_{i, t} \geq 0$ respectively. Moreover, the non-linear terms $\mu_{i, t} \cdot q_{i, t}$ and $\lambda_{i, t} \cdot\left(q_{i, t}-P C_{i, t}\right)$ could be linearized, which in turn yields the linearized $K K T$ condition as below:

The linearized K.K.T conditions of the equilibrium price model $(t)$

$$
\begin{array}{lc}
\left(\delta_{i, t}+t c r^{i, t}\right)+q_{i, t} \cdot \theta_{i, t}+E_{t} \cdot \theta_{i}-\theta_{i} \cdot\left(E_{t}-\alpha_{t} \cdot p_{t}\right)-\lambda_{i, t}+\mu_{i, t}=0 & \forall i \\
0 \leq q_{i, t} \leq P C_{i, t} & \forall i \\
\lambda_{i, t} \leq M_{1} \cdot v_{i, t} & \forall i \\
\left(q_{i, t}-P C_{i, t}\right) \leq M_{1} \cdot\left(1-v_{i, t}\right) & \forall i \\
\mu_{i, t} \leq M_{2} \cdot u_{i, t} & \forall i \\
q_{i, t} \leq M_{2} \cdot\left(1-u_{i, t}\right) & \forall i \\
d_{i}=\sum_{i} q_{i, t}=E_{t}-\alpha_{t} \cdot p_{t} & \forall i \\
v_{i, t}, u_{i, t} \in\{0,1\} & \forall i
\end{array}
$$

The profit of a firm will be increased if its transaction cost is decreased. This is due to the fact that in such cases, if the firm does not change its production quantity and just produces as the previous level, then its production cost will be decreased while their income has increased. Also, if it finds some opportunities for changing the production quantity, it will certainly follow this change for seeking more profit. The overall effect is that its profit is increased. On the other hand, it could be proved that when a firm's transaction cost is decreased while other firms' transaction costs remain fixed, the equilibrium price of the market is decreased or remains at the previous level. This shows better utility for customers if the formation of an alliance with the reduced transaction cost is advocated by the local government. The proof of this as Proposition 4.1 is presented in Appendix 4.1. 
Proposition 4.1: The equilibrium price of the market will either decrease or remain at the previous price as the transaction cost of a firm decreases.

For estimating the conjecture price of each firm, a method called advanced implicit estimation is used in this chapter. This method only uses information related to the price and supplied quantity of firms (de Haro et al., 2007). Moreover, the method does not use other information such as profit margins or costs of manufacturers. Thus, it is possible to apply it to markets with low disclosed information. The interested reader could refer to de Haro et al. (2007) for more information about the method.

\subsubsection{Net income before and after taxation}

Taxation is another important factor affecting the profitability of firms. Generally, tax is levied at a predetermined rate on the taxable incomes, i.e., sales revenue of the company minus total production costs, depreciation expense, and loan interest costs. Some accounting standards differentiate between revenue on cash by the invoiced sales. However, in the present study, it is assumed that these are the same. On the other hand, if the taxable income is negative in one period, the negative taxable income is recorded as the accumulated loss to reduce tax in subsequent periods.

Since the tax rate of foreign investors is different from that of local investors in some countries, the taxed incomes of the licensor and licensee are calculated separately in this chapter. Therefore, if we consider the introduced notations, the equations below are used to address the tax-related constraints of firms in the alliance.

\section{The tax constraint $(T)$}

$$
\begin{array}{ll}
S_{L F, t}=\left(1-r_{f}\right) \cdot p_{t} \cdot q_{L F, t}-\left(\delta_{L F, t}+t c r^{J V, t}\right) \cdot q_{L F, t}-D P_{L F, t} & \forall t \in T, t \geq 1 \\
T I_{L, t}=(1-\varphi) \cdot\left(S_{i, t}-r d \cdot I_{t}\right) & \forall t \in T, t \geq 1 \\
T I_{F, t}=\varphi \cdot\left(S_{i, t}-r d \cdot I_{t}\right) & \forall t \in T, t \geq 1
\end{array}
$$

Eq. (4.31) shows the net income of the alliance before interest and taxes, which is composed of sales income minus the production and transaction costs minus the depreciation cost minus the license income paid by the alliance to the main firm of the licensor. Eq. (4.32) and (4.33), respectively, relate the taxable dividend incomes of the licensee and licensor to their share on the alliance ownership. 
Regarding the tax constraint $(T)$, the net taxable dividend incomes of licensor and license are determined according to Eqs. (4.34) - (4.37).

$$
\begin{array}{ll}
A L_{j, 0}=0 & \forall t \geq 1, j \in\{L, F\} \\
T I_{j, 0}=0 & \forall t \geq 1, j \in\{L, F\} \\
A L_{j, t}=\max \left(0, A L_{j, t-1}-T I_{j, t}\right) & \forall t \geq 1, j \in\{L, F\} \\
T X_{j, t}=\max \left(0, T I_{j, t}-A L_{j, t-1}\right) & \forall t \geq 1, j \in\{L, F\}
\end{array}
$$

Eqs. (4.36) and (4.37) could be linearized using the following equations:

\section{The linearized tax constraints set $(j, T)$}

$$
\begin{array}{ll}
A L_{j, t-1}-T I_{j, t}-M \cdot g_{j, t} \leq 0 & \forall t \in T \\
A L_{j, t-1}-T I_{j, t}+M \cdot\left(1-g_{j, t}\right) \geq 0 & \forall t \in T \\
0 \leq A L_{j, t} \leq M \cdot g_{j, t} & \forall t \in T \\
A L_{j, t}-M \cdot\left(1-g_{j, t} \leq A L_{j, t-1}-T I_{j, t} \leq A L_{j, t}\right. & \forall t \in T \\
0 \leq T X_{j, t} \leq M \cdot\left(1-g_{j, t}\right) & \forall t \in T \\
T X_{j, t} \leq\left(T I_{j, t}-A L_{j, t-1}\right) \leq T X_{j, t}+M \cdot\left(g_{j, t}\right) & \forall t \in T \\
T I_{j, t} \quad \text { free } & \forall t \in T \\
g_{j, t} \in\{0,1\} & \forall t \in T
\end{array}
$$

\subsubsection{The deterministic model for capacity planning and optimal arrangement of a proportional contract}

By manufacturing the products domestically using the license contract, the transaction cost of the resulting alliance will be reduced as one of the benefits of the cooperation. Also, as proven in Proposition 4.1, the equilibrium price might be decreased, and consequently, the alliance might obtain a large share in the local market. Considering the efficiency of the licensor and licensee, as illustrated in the base model of the thesis in chapter 3 , the parameters of the license contract and the other decision variables should be stipulated such that the profit of partners is maximized. So, taking into account the above descriptions, the following model should be investigated to address the mentioned problem:

$$
\max \pi_{F}-\pi_{S P, F}
$$

s.t. 


$$
\begin{array}{ll}
\pi_{F}-\pi_{S P, F}=\pi_{L}-\pi_{S P, L} & \\
N C_{L, t}=(1-\varphi)\left(S_{L F, t}-r d \cdot I_{t}-V_{t}+D P_{t}\right)+\tau_{L} \cdot T X_{L, t} & \forall t \in T, t \geq 1 \\
N C_{F, L}=\varphi \cdot\left(S_{L F, t}-r d \cdot I_{t}-V_{t}+D P_{t}\right)+\tau_{F, 2} \cdot T X_{F, t}+\left(1-\tau_{F, 2}\right) \cdot r_{f} \cdot p_{t} \cdot q_{L F, t} & \forall t \in T, t \geq 1 \\
\pi_{F}=\sum_{t \geq 1} N C_{F, t} \cdot(1+r)^{-t}+\varphi \sum_{t \geq 0}\left(S B V_{t}-S I_{t}\right) \cdot(1+r)^{-t}-N C_{F, 0} & \\
\pi_{L}=\sum_{t \geq 1} N C_{L, t} \cdot(1+r)^{-t}+(1-\varphi) \cdot \sum_{t \geq 0}\left(S B V_{t}-S I_{t}\right) \cdot(1+r)^{-t}-N C_{L, 0} & \\
0 \leq q_{L F, t} \leq P C_{L F, t} & \forall t \in T
\end{array}
$$

The linearized K.K.T conditions of the equilibrium price model $(t) \quad \forall t \in T, t \geq 1$

The depreciation evaluation constraints set $(T)$

The Capacity Generation Constraint sets $(T)$

The Loan evaluation constraints set $(T)$

The tax constraint $(T)$

The linearized tax constraints set $(L, T)$

The linearized tax constraints set $(F, T)$

Eq. (4.39) expresses the objective function of the model, as discussed earlier. Based on Eq. (4.40) and as a base assumption of the proportional contract, the gain of cooperation should be split between the licensor and licensee equally. Eqs. (4.41) and (4.42) represent the net cash flow of the licensee and licensor in the production periods, respectively. It should be pointed out that the cash flow calculation in the model is based on the Free Cash Flow to Equity ( FCFE) valuation method, which adds depreciation to the partner's share of net income and reduces the interest, tax, and principles of loan from it. Eqs. (4.43) and (4.44) discount the cash flows of the licensor and licensee to time zeros to compute their profit in the alliance. Eq. (4.45) restricts the production quantity of the alliance to its capacity level. Finally, Eqs. (4.46) - (4.52) presents the constraint sets argued earlier.

\subsubsection{The two-stage stochastic model for capacity planning and optimal arrangement of a proportional contract}

Due to the uncertain nature of some parameters of the model, such as the capacity of other firms in the market or the demand function of the product, the two-stage stochastic programming method is utilized in this chapter. This method is proper for the proposed model because the capacity level and contract-related variables should be decided at the first stage and before any realization of uncertain events. Other variables will be decided appropriately after the realization of the uncertain events. Also, the scenario generation of the model, which 
is explained later, is in line with the scenario-based valuing methods such as the simple leastsquares approach of Longstaff and Schwartz (2004).

Denoting $x$ as the vector of first-stage variables, $\Omega$ as the index of generated scenarios, $y(\Omega)$ as the vector of the second-stage variables for each scenario, $c^{T}$ and $b$ as vectors of known parameters, $A$ as a matrix of known parameters, $q^{T}(\Omega)$ and $h(\Omega)$ as vectors of uncertain parameters, $T(\Omega)$ and $W(\Omega)$ as matrixes of uncertain parameters, and $p(\Omega)$ as the probability of scenario $\Omega$, the two-stage stochastic programming model could be represented as the following (Dantzig \& Infanger, 1995):

$$
\begin{aligned}
& \min _{\text {s.t. }} z=c^{T} x+E_{\Omega}\left[\min q(\Omega)^{T} y(\Omega)\right]=c^{T} x+\sum_{\Omega} p(\Omega) \cdot q(\Omega)^{T} y(\Omega) \\
& A x=b \\
& T(\Omega) x+W y(\Omega)=h(\Omega) \quad \forall \Omega \\
& x \geq 0 \\
& y(\Omega) \geq 0 \quad \forall \Omega
\end{aligned}
$$

In the proposed model, the capacity level of alliance $P C_{L F, 0}$, the license contract parameters namely, $\varphi$ and $r_{f}$, the loan and depreciation-related variables $\left(V_{t}, I_{t}\right.$ and $\left.D P_{t}\right)$, and the founding of licensor and licensee at the beginning of the alliance $\left(N C_{L, 0}, N C_{F, 0}\right)$ are the first-stage variables while other variables are the second-level or scenario-based variables. The set of all scenarios is denoted by $\Psi$, and $p(\Omega)$ is considered as the probability of occurring scenario $\Omega \in \Psi$. Moreover, the uncertain parameters are $\delta_{i, t, \Omega}, \theta_{i, t, \Omega}$ and $t c r_{i, t, \Omega}$, which are related to other firms and have some level of uncertainty, and the parameters of the market, i.e. $\theta_{i, t, \Omega}, d_{t}$ . Therefore, each of the model constraints, which have at least one scenario-based variable or one scenario-based parameters is redefined over all the generated scenarios. Accordingly, the uncertain model is introduced as follows:

$$
\begin{array}{ll}
\max & \pi_{F}-\pi_{S P, F} \\
\text { s.t. } & \pi_{F}-\pi_{S P, F}=\pi_{L}-\pi_{S P, L} \\
& N C_{L, t, \Omega}=(1-\varphi)\left(S_{L F, t, \Omega}-r d \cdot I_{t}-V_{t}+D P_{t}\right)+\tau_{L} \cdot T X_{L, t, \Omega} \forall t \in T, t \geq 1, \Omega \in \Psi \\
& N C_{F, L, \Omega}=\varphi \cdot\left(S_{L F, t, \Omega}-r d \cdot I_{t}-V_{t}+D P_{t}\right)+\tau_{F, 2} \cdot T X_{F, t, \Omega}+\left(1-\tau_{F, 2}\right) \cdot r_{f} \cdot p_{t, \Omega} \cdot q_{L F, t, \Omega} \\
& \forall t \in T, t \geq 1, \Omega \in \Psi \\
& \pi_{F}=\sum_{\Omega} p(\Omega) \cdot\left(\sum_{t \geq 1} N C_{F, t} \cdot(1+r)^{-t}+\varphi \sum_{t \geq 0}\left(S B V_{t}-S I_{t}\right) \cdot(1+r)^{-t}-N C_{F, 0}\right)
\end{array}
$$




$$
\begin{aligned}
& \pi_{L}=\sum_{\Omega} p(\Omega)\left(\sum_{t \geq 1} N C_{L, t} \cdot(1+r)^{-t}+(1-\varphi) \cdot \sum_{t \geq 0}\left(S B V_{t}-S I_{t}\right) \cdot(1+r)^{-t}-N C_{L, 0}\right) \\
& 0 \leq q_{L F, t, \Omega} \leq P C_{L F, t, \Omega}
\end{aligned}
$$

The linearized K.K.T conditions of the equilibrium price model $(t, \Omega)$

$$
\forall t \in T, t \geq 1, \Omega \in \Psi
$$

The Depreciation evaluation constraints set $(T, \Omega)$

The Capacity Generation Constraint sets $(T, \Omega)$

The Loan evaluation constraints set $(T, \Omega)$

The tax constraint $(T, \Omega)$

The linearized tax constraints set $(L, T, \Omega)$

The linearized tax constraints set $(F, T, \Omega)$

$$
\begin{array}{ll}
N C_{L, 0, \Omega}=N C_{L, 0, \Omega^{\prime}} & \forall \Omega, \Omega^{\prime} \in \Psi \\
N C_{F, 0, \Omega}=N C_{F, 0, \Omega^{\prime}} & \forall \Omega, \Omega^{\prime} \in \Psi \\
P C_{L F, 0, \Omega}=P C_{L F, 0, \Omega^{\prime}} & \forall \Omega, \Omega^{\prime} \in \Psi
\end{array}
$$

A remarkable note about the uncertain model is that Eqs. (4.68) - (4.70) are introduced to make the variable $N C_{L, t, \Omega}, N C_{F, t, \Omega}$, and $P C_{L F, t, \Omega}$ independent of the scenarios in period zero though in the remaining period, they are scenario-dependent.

It should also be noted that the non-linear expression $p_{t, \Omega} . q_{L F, t, \Omega}$ in the model can be converted to a linear expression with the following modifications. First, we should replace the variable $q_{L F, t, \Omega}$ with the variable $q_{L F, t, \Omega}=\underline{q}_{L F, t, \Omega}+\nabla_{L F, t, \Omega} \cdot \sum_{k} 2^{k} \cdot b_{L F, t, k, \Omega}$ (Pereira et al., 2005). In this respect, $\underline{q}_{L F, t, \Omega}$ is the lower limit of the variable $q_{L F, t, \Omega}, \nabla_{L F, t, \Omega}$ is the production quantity step size, $k$ is the set of discretization intervals, and $b_{L F, t, k, \Omega}$ is a binary variable. Also, the variable $v_{L F, t, k, \Omega}$ is defined in the form of $v_{L F, t, k, \Omega}=b_{L F, t, k, \Omega} \cdot p_{t, \Omega}$ which is linearized by the following equations:

$$
\begin{array}{ll}
0 \leq v_{i, t, k, \Omega} \leq M . b_{i, t, k, \Omega} & \forall i, t, k, \Omega \\
v_{i, t, k, \Omega} \leq p_{t, \Omega} \leq v_{i, t, k, \Omega}+M .\left(1-b_{i, t, k, \Omega}\right) & \forall i, t, k, \Omega
\end{array}
$$

To find the equilibrium price of the market, the above model could be solved for all manufacturers simultaneously. Such problems are identified as equilibrium problems with equilibrium constraints (EPEC). There are some solution approaches to solve these problems but with a small number of variables (Moisiva et al., 2017). However, estimating the 
parameters as well as competitors' decisions, which is a kind of long-term decision, is very difficult. Therefore, in the present study, instead of an EPEC approach that is very complex and cannot be guaranteed to reach a Nash equilibrium (Moisiva et al., 2017), we solve this problem only for the concerned alliance. Instead, it is assumed that other partners' decisions, such as their capacity level, are a random variable, and by creating different scenarios, we consider the other firms' capacity levels as scenario-dependent parameters.

\subsubsection{Scenario generation}

This thesis aims to valuation the strategic alliance with mathematical programming, so the assumptions reflect the market model. According to market theory, path-dependent and market shock are the most important assumptions considered in the thesis. Path-dependant means that the future behavior of the customers of a product is the function of their past decisions, which can be stochastic. An example given by the analysis of the dynamic system in this regard is that the dependence of users of Windows OS, which the introduction of new OS can not eliminate the previous history and interests of customers in OS. Considering the market shock in a product's market is also due to the introduction of new products that can distort the market of an existing product. For example, in the field of car tires without the need for tubes, which has fundamentally changed the market of tube manufacturing companies.

The notation $\tilde{x}_{i t}$ is used to indicate the uncertain parameter $i$ in period $t$ that has normal distribution with mean $\mu_{i}$ and standard deviation $\sigma_{i}$. Moreover, the value of the uncertain variable $i$ in period $t$ and in the scenario $\Omega$ is expressed by $x_{i t \Omega}$. The value of parameters in scenarios could be generated using Eq. (4.73) and (4.74) (Vahdat and Vahdatzad, 2017):

$$
\begin{array}{lr}
x_{i t \Omega}=\mu_{i}+\hat{\varepsilon}_{i t \Omega} & \forall t, \Omega \\
\hat{\varepsilon}_{i t \Omega}=\sum_{j=1}^{P} \beta_{j} \hat{\varepsilon}_{i(t-j) \Omega}+\varepsilon_{i t \Omega} & \forall t, \Omega
\end{array}
$$

Where $\varepsilon_{i t \Omega}$ is a random variable with the normal distribution, mean zero, and standard deviation $\sigma_{i}$. Eq. (4.74) stands for the path dependency property of variables which is relevant in generating the random demand of markets over time. However, if a random variable is independent of its precedent periods, the parameter $\beta_{j}$ in Eq. (4.74) should be set equal to zero to reflect this fact. 
Based on Eqs. (4.73) and (4.74), the values of the random variable $\varepsilon_{i t \Omega}$ in each scenario should be determined. Also, if the numbers of intended scenarios and planning periods are denoted by $n_{1}$ and $n_{2}$ respectively, then $n_{n}=n_{1} \times n_{2}$ samples should be extracted from the probability distribution to determine the values of the random variable in the scenarios. Considering $k$ random variables in the problem, the extracted samples should be as for Eq. (4.75), Where $x_{\mathrm{ij}}$ is the $i$-th random sample (regardless of the order of the sample generated from the random variable) of the random variable $\tilde{x}_{j}$.

$$
\left\{x_{n 1}, x_{n 2}, \ldots, x_{n k}\right\}, \ldots,\left\{x_{21}, x_{22}, \ldots, x_{2 k}\right\},\left\{x_{11}, x_{12}, \ldots, x_{1 k}\right\}
$$

Next, the Latin hypercube sampling $(L H S)$ method is used to simulate random variables (Olsson et al., 2003). In this method, it is assumed that the probability distribution function of random variables is known or that it could be derived from previous samples of the random variables. In this method, the matrix $P$ as an $n \times k$ dimensional matrix is used where each of its columns contains a randomly generated permutation of integers from 1 to $n$. Furthermore, $R$ is regarded as a matrix with the same dimensions of $P$ whose elements are uniformly generated real numbers in the interval $[0,1]$. Using $P$ and $R$, the matrix $S$ is formed as Eq. (4.76).

$$
S=\frac{1}{N}(P-R)
$$

Bearing in mind the matrix $S$, the $i$-th sample of $j$-th random variable is taken using Eq. (4.77) where $F_{x_{j}}^{-1}$ is the inverse function of the cumulative distribution of the random variable $\tilde{x}_{j}$.

$$
x_{i j}=F_{x_{i}}^{-1}\left(s_{i j}\right)
$$

It should also be pointed out that the demand function in this chapter is in the form of Eq. (4.73), i.e., $d_{t}=E_{t}-\alpha_{t} \cdot p_{t}+\varepsilon_{t}$. Here, $\varepsilon_{t}$ is a normal random variable with mean zero and standard deviation $\sigma_{t}$. This means that the uncertainty in demand is regarded as a whole instead of considering uncertainty in $E_{t}$ and $\alpha_{t}$. Notably, the distribution of all uncertain parameters is estimated using historical data.

\subsection{Solution approach}

First, the stand-alone profit of the partners in the planning horizon in the case of noncooperation should be clarified. In the base model, it was stated that the stand-alone profit is proportional to the partners' investment in the alliance, and the return on the capital rate (ROC) 
could be used to satisfy this statement. Therefore, referring to the length of the planning horizon as $T$, the stand-alone profits of the partners are estimated as Eqs. (4.78) and (4.79):

$$
\begin{aligned}
& \pi_{S P, F}=N C_{F, 0} \cdot\left(\left(\frac{1+R O C_{F}}{1+r}\right)^{T}-1\right) \\
& \pi_{S P, \gg}=N C_{L, 0} \cdot\left(\left(\frac{1+R O C_{L}}{1+r}\right)^{T}-1\right)
\end{aligned}
$$

In Eqs. (4.78) and (4.79), it is presumed that if partners invest in their own business instead of the alliance, they could receive $(1+R O C)$ per each unit of their investment. However, their profit should be discounted to period zero by rate $r$ to reflect the time value of money on a similar scale for both partners.

It is notable that the above model could be solved for small-size problems using commercial software such as GAMS. However, for large-scale problems that are more relevant to reality, this software will not be able to solve the problem in an acceptable time. So, a procedure based on the backward dynamic programming method is proposed to solve the problem efficiently. In the proposed procedure, the following notes are taken into account:

1) The return on capital $(R O C)$ of the partners is higher than the interest rates $r$ and $r d$. This is because otherwise, the business of the partners is not affordable or profitable. In addition to the lower interest rate of the loan than the $R O C$ rate, the loan has a taxreduction effect as declared in the model. Therefore, providing the required investment of the alliance through the loan is optimal as far as possible.

2) If it is decided to terminate the alliance in one period, the depreciation cost in that period will not be reduced from the book value of the capacity. Moreover, due to the payment of the remaining principal of the loan, the interest cost is not incurred at the liquidation period. On the other hand, by continuing the alliance in a period, the depreciation and the interest cost could be regarded as the investment cost expended in the period. With this vision, the one-period horizon in the base model and its properties are utilized in the backward dynamic programming procedure to decide whether or not to terminate the alliance in a period.

3) In the proposed procedure, the capacity level will be chosen from several alternatives in a discrete solution space. Hence, the quality of solutions is dependent on the available alternatives. However, this initiative and the first note simplify the problem because the capacity and loan-related variables will be fixed in doing so. 
4) Also, as proved in the base model, the pricing decision should be taken in a way that the profit of the alliance as a whole is maximized, and the optimal pricing decisions do not depend on the license contract parameters. So, after identifying the capacity level at the beginning of the alliance ( $t=0)$, the equilibrium price model $(t)$ in Eq. (4.28) is solved to find the equilibrium price of the market in each period.

In implementing the backward dynamic programming procedure, the definitions of " ideal gain function" and " marginal free-tax operational gain on the share" (MGS) from the base model are borrowed. For this purpose, the ideal gain functions of the licensor $\left(O A_{F}\right)$ and licensee $\left(O A_{L}\right)$ are defined based on Eqs. (4.80), and (4.81).

$$
\begin{array}{ll}
O A_{F}(\Omega, T+1)=O A_{L}(\Omega, T+1)=0 & \forall \Omega \\
O A_{F}(\Omega, t)=\sum_{t^{\prime}=t+1}^{T+1} O A_{F}\left(\Omega, t^{\prime}\right) \cdot(1+r)^{-t^{\prime}}+\left(1-\tau_{F, 2}\right) . & \\
\left(\left(p_{t, \Omega}-\left(t c r_{L F, t, \Omega}+\delta_{L F, t, \Omega}\right)\right) \cdot q_{L F, t, \Omega}-D P_{t}-r d \cdot I_{t}\right)-\left(R O C_{F}\right) \cdot\left(D P_{t}+r d \cdot I_{t}\right) & \forall t \leq T, \Omega \\
O A_{L}(\Omega, t)=\sum_{t^{\prime}=t+1}^{T+1} O A_{L}\left(\Omega, t^{\prime}\right) \cdot(1+r)^{-t^{\prime}}+\left(1-\tau_{L}\right) . & \\
\left(\left(p_{t, \Omega}-\left(t c r_{L F, t, \Omega}+\delta_{L F, t, \Omega}\right)\right) \cdot q_{L F, t, \Omega}-D P_{t}-r d \cdot I_{t}\right)-\left(R O C_{L}\right) .\left(D P_{t}+r d . I_{t}\right) & \forall t \leq T, \Omega
\end{array}
$$

In the base model, it is proved that the necessary and sufficient condition for the formation of an alliance is $O A_{L} \geq 0$. In our multi-period model and the backward dynamic programming approach, that property is equivalent to saying that the necessary and sufficient condition for liquidating the alliance in a period of a scenario is that $O A_{L}(\Omega, t)<0$. Furthermore, as proven in the based model, the choice between the shared ownership structure and pure licensee ownership depends on the sign of the " marginal free-tax operational gain on the share" $(M G S)$. The non-negativity of $M G s$ indicates that the shared ownership structure is optimal, while negative values of $M G s$ prove that the licensor income from the alliance should be just in the form of a royalty fee. As Eq. (4.83) shows, MGs only depend on the return on capital and the tax rate of partners, and therefore, the ownership structure of an alliance could be decided optimally without solving the model.

$$
M G_{S}=\left[\frac{R O C_{L}}{1-\tau_{L}}-\frac{R O C_{F}}{1-\tau_{F, 2}}\right] . D P
$$

In the case of $M G s<0$, the optimal solution will be such that the ownership share of the licensor is zero, i.e., $\varphi=0$ and the royalty rate $r_{f}$ is determined such that the Eq. (4.84) holds:

$$
L P=r_{f} \cdot \sum_{\Omega} p(\Omega) \cdot \sum_{t=1}^{T} p_{t \Omega} \cdot q_{L F, t, \Omega} \cdot O_{t \Omega}=\frac{O A_{L}}{2-\tau_{L}-\tau_{F, 1}}
$$


On the other hand, when $M G_{s} \geq 0$ the shared ownership is optimal, the license income is zero $\left(r_{f}=0\right)$, and the optimal share of the licensor in the ownership of alliance will be as Eq. 4.85:

$$
\varphi^{*}=\frac{O A_{L}}{O A_{L}+O A_{F}}
$$

By determining the optimal value of $\varphi^{*}, O A_{F}$ and $O A_{L}$ the optimal gain of the partners from the partnership ( $\pi$ ) in the alliance is identified as Eq. (4.86):

$$
\pi=\pi_{F}-\pi_{S P, F}=\pi_{L}-\pi_{S P, L}=\varphi \cdot O A_{F}-\varphi \cdot\left(1-\tau_{F, 2}\right) \cdot L P+\left(1-\tau_{F, 2}\right) \cdot L P
$$

Denoting the discrete set of capacity level alternatives by $P C$, the backward dynamic programming solution approach for solving the model is presented as follows:

\section{The dynamic programming solution approach}

1) For each capacity level indexed by $k$ in $P C$ :

a. Estimate the cost of capacity generation using Eq. (4.9).

b. Estimate the loan using Eqs. 4.9 and 4.10.

c. Estimate $I_{t}$ using Eqs. 4.17- 4.19.

d. Estimate $D P_{t}$ using Eqs. 4.2- 4.4.

e. For each scenario $\Omega$ do:

i. Set $O A_{F}(\Omega, T+1)=0$ and $O A_{L}(\Omega, T+1)=0$

ii. Set $t=T$

iii. Compute $O A_{L}(\Omega, t)$ using Eq. (4.70).

1. If $O A_{L}(\Omega, t)<0$, set $O A_{L}(\Omega, t)=0, O A_{F}(\Omega, t)=0$ and liquidate the alliance i.e., set $O_{t \Omega}=0$.

2. Otherwise, set $O_{t \Omega}=1$

iv. If $\mathrm{t}==0$

1. Set $O A_{L}(k, \Omega)=O A_{L}(\Omega, 0), O A_{F}(k, \Omega)=O A_{F}(\Omega, 0)$.

v. Otherwise, set $\mathrm{t}=\mathrm{t}-1$ and go to step iii.

f. Set $O A_{L}(k)=\sum_{\Omega} p(\Omega) \cdot O A_{L}(k, \Omega)$ and $O A_{F}(k)=\sum_{\Omega} p(\Omega) \cdot O A_{F}(k, \Omega)$.

g. Set $O A_{L}=O A_{L}(k), O A_{F}=O A_{F}(k)$.

h. If $M G s<0$

i. set $\varphi(k)=0$ and $L P(k)=L P$ in Eq. (4.84).

i. Else

i. Set LP $(\mathrm{k})=0$ and $\varphi(k)=\varphi$ in Eq. (4.85).

j. Compute $\pi$ in Eq. (4.86) by setting $O A_{L}=O A_{L}(k), O A_{F}=O A_{F}(k), \varphi(k)=\varphi$ , $L P(k)=L P$ and then set $\pi(k)=\pi$. 
2) Select the optimal level of capacity $k^{*}$ as the level which has the maximum value for $\pi(k)$. By determining $k^{*}$, we have $\varphi^{*}=\varphi(k), L P^{*}=L P\left(k^{*}\right), O A_{F}^{*}=O A_{F}\left(k^{*}\right)$ and $O A_{L}^{*}=O A_{L}\left(k^{*}\right)$.

\subsection{Numerical results}

In this section, the case study is first described, and the results of implementing the model are explained. Second, a sensitivity analysis is carried out to examine the model behaviors for different values of considered parameters.

\subsubsection{The case study}

As the case study, the production of Citric Acid under the license of a foreign firm in Iran is investigated. Citric acid is used in the beverage, juice, cosmetics, and pharmaceutical industries, and in addition to flavoring, it also regulates $\mathrm{PH}$. This product is homogenous and is mainly imported from a few foreign manufactures. The proposed model is used to validate the feasibility of the domestic production of the product.

The data about the demand, production cost, and selling price are provided from the Iran Chamber of Commerce, Industries, Mines, and Agriculture. The collected data is from 2005 to 2019. We found eight suppliers that supply the product according to Table (4.1). The data reveal an increasing trend in the supply of the product over the considered periods. The estimated production cost of suppliers is as Table 4.2. Moreover, the transaction cost comprises the transportation cost, the sum of customs duties and commercial interest (4\%), and VAT (9 $\%$ ) which are according to the local market laws. The transaction costs of suppliers in different years are represented in Table 4.3. Also, the market prices as the equilibrium prices are shown in Table 4.4. It is estimated that a capacity level for the production of 10000 tons costs $\$ 23$ million. Therefore, the cost of each unit of capacity will be $\$ 2.3$. On the other hand, another cost of accounting the non-depreciable part of the capacity is assessed to be equal to $\$ 0.22$ per each unit of capacity. At the time of liquidation, the remaining value of the capacity is considered equal to its book value. Considering the demand and market price in Tables 4.1 and 4.4 and using the least squared method, the demand function is estimated as Figure 4.2. As mentioned, the method of de Haro et al. (2007) is used to calculate the conjecture price of suppliers who have positive supplies each year using the data in Tables 4.1-4.4. The estimation is started by setting $\theta_{i, t}^{0}=1 / \alpha$ and leads to the conjecture prices according to Table 4.5. 
In the case problem, supplier seven has been chosen as the licensor of the product. By forming the alliance, the transaction cost of the alliance decreases significantly, and we set $t c r^{L F, t}=0$ as one of the cooperation benefits. As mentioned, the capacity of the alliance is a first-level decision variable. However, the capacity level and the transaction cost of other suppliers as well as the conjecture price, the production cost of all suppliers (including the alliance), and the error term of demand function are regarded as uncertain parameters. We assume that the uncertain parameters have a normal distribution. Accordingly, the data of Tables 4.1-4.5 are used to estimate the mean and the variance of uncertain parameters. Then, the scenario generation method is used to generate the value of the uncertain parameters in each scenario. In this problem, the planning horizon after the formation of the alliance is 20 years, and 1000 scenarios are generated. More precisely, the shock scenarios are applied in $16 \%$ of the generated scenarios randomly. The shock scenarios are ones wherein the supply of the products is stopped at one period, and there is no market for the product after that. The stoppage time in the shock scenarios is also selected randomly with uniform distribution. This assumption is very common in real-world problems and makes the proposed model in line with the patent valuation method, such as Longstaff and Schwartz (2001).

For encouraging foreign investment in the local market, the corporate tax rate of local firms and foreign firms in alliances is set equally in Iran. This rate is $25 \%$, and consequently, we have $\tau_{L}=\tau_{F, 2}=0.25$. However, the tax rate on the license income is greater than the corporate tax rate, and in the considered case study, we have $\tau_{F, 1}=0.3$. Moreover, investigating accounting statements of firms, it is revealed that $R_{O} O C_{F}=0.29, R O C_{L}=0.25$ and $r=0.2$. Therefore, the $M G s$ in Eq. (4.83) is negative, and the maximum share of the local firm in the alliance ownership is optimal, i.e., $\varphi^{*}=0$.

Now, using the proposed dynamic programming-based procedure, the gains of partners (Eq. 4.40) for various capacity levels are obtained as Table 4.6. The results indicate that the capacity level $P C_{L F, 0}=8000$ tons is optimal. Also, considering the net present value of the sales income, the royalty rate of $4.577 \%$ is proposed. This royalty rate is not far from the real and reported royalty rate in pharmaceutical products. However, as stated in the proposed model, many realworld aspects and competitive advantages of the partners have been regarded to reach this rate. 
Table 4.1. The amount of supply in previous years (Kilogram)

\begin{tabular}{|c|c|c|c|c|c|c|c|c|c|c|c|c|c|c|c|}
\hline $\begin{array}{l}\text { Period } \\
\text { supplier }\end{array}$ & 2005 & 2006 & 2007 & 2008 & 2009 & 2010 & 2011 & 2012 & 2013 & 2014 & 2015 & 2016 & 2017 & 2018 & 2019 \\
\hline 1 & 151000 & 852511 & 1009698 & 1009698 & 2067659 & 2822028 & 345554 & 2225066 & 523396 & 0 & 1302454 & 0 & 0 & 0 & 0 \\
\hline 2 & 518578 & 254420 & 65220 & 65220 & 302649 & 461349 & 1315468 & 621358 & 348249 & 1775329 & 1252861 & 0 & 2824862 & 2745301 & 1349712 \\
\hline 3 & 480727 & 794317 & 563245 & 563245 & 1315598 & 1920211 & 194350 & 1235732 & 1194987 & 271177 & 122584 & 250521 & 438975 & 87738 & 28500 \\
\hline 4 & 296267 & 34000 & 156272 & 156272 & 0 & 50472 & 0 & 0 & 120000 & 127512 & 547893 & 1305262 & 18708 & 61049 & 44665 \\
\hline 5 & 2114 & 18426 & 215095 & 215095 & 0 & 161771 & 17513 & 0 & 0 & 0 & 0 & 0 & 0 & 627488 & 744405 \\
\hline 6 & 0 & 0 & 0 & 0 & 40000 & 26299 & 1563221 & 1537570 & 2803969 & 1808756 & 1737340 & 6501468 & 8177945 & 8078469 & 8179889 \\
\hline 7 & 0 & 0 & 0 & 0 & 0 & 59254 & 1423568 & 0 & 0 & 0 & 1985242 & 0 & 42325 & 53307 & 18324 \\
\hline 8 & 0 & 0 & 0 & 0 & 0 & 0 & 1230250 & 1209504 & 2426893 & 4653132 & 232656 & 856559 & 993604 & 0 & 43382 \\
\hline Sum & 1448686 & 1953674 & 2009530 & 2009530 & 3725906 & 5501384 & 6089924 & 6829230 & 7417494 & 8635906 & 5181030 & 8914460 & 12496419 & 11653352 & 10408877 \\
\hline
\end{tabular}

Table 4.2. The production cost of supplier in previous years (\$ / Kilogram)

\begin{tabular}{cccccccccccccccc}
\hline $\begin{array}{c}\text { Period } \\
\text { supplier }\end{array}$ & $\mathbf{2 0 0 5}$ & $\mathbf{2 0 0 6}$ & $\mathbf{2 0 0 7}$ & $\mathbf{2 0 0 8}$ & $\mathbf{2 0 0 9}$ & $\mathbf{2 0 1 0}$ & $\mathbf{2 0 1 1}$ & $\mathbf{2 0 1 2}$ & $\mathbf{2 0 1 3}$ & $\mathbf{2 0 1 4}$ & $\mathbf{2 0 1 5}$ & $\mathbf{2 0 1 6}$ & $\mathbf{2 0 1 7}$ & $\mathbf{2 0 1 8}$ & $\mathbf{2 0 1 9}$ \\
\hline $\mathbf{1}$ & 0.764 & 0.7448 & 0.7472 & 0.7784 & 0.9732 & 0.8128 & 0.768 & 0.974 & 0.8884 & 0 & 0.926 & 0 & 0 & 0 & 0 \\
$\mathbf{2}$ & 0.7036 & 0.7512 & 0.754 & 0.8748 & 1.1284 & 1.1212 & 1.0848 & 1.0484 & 0.8776 & 0.8376 & 0.7724 & 0 & 0.5408 & 0.5896 & 0.664 \\
$\mathbf{3}$ & 0.7324 & 0.778 & 0.808 & 0.7988 & 1.1068 & 1.0604 & 0.928 & 1.1876 & 0.972 & 0.9392 & 0.8584 & 0.644 & 0.8404 & 1.0128 & 0.9656 \\
$\mathbf{4}$ & 0.6724 & 0.7672 & 0.752 & 0.6156 & 0 & 0.8276 & 0 & 0 & 1.1204 & 0.5032 & 0.7428 & 0.5952 & 0.5448 & 0.4488 & 0.8524 \\
$\mathbf{5}$ & 0.6516 & 0.7272 & 0.7676 & 0 & 0 & 1.0764 & 0.908 & 0 & 0 & 0 & 0 & 0 & 0 & 1.054 & 0.9076 \\
$\mathbf{6}$ & 0 & 0 & 0 & 0 & 1.0184 & 0.5904 & 0.7588 & 0.8756 & 0.8512 & 0.8316 & 0.6276 & 0.462 & 0.504 & 0.572 & 0.6568 \\
$\mathbf{7}$ & 0 & 0 & 0 & 0 & 0 & 0.8792 & 0.8564 & 0 & 0 & 0 & 0.7828 & 0 & 1.172 & 0.9288 & 0.8444 \\
$\mathbf{8}$ & 0 & 0 & 0 & 0 & 0 & 0 & 0.97 & 1.084 & 0.9796 & 0.8736 & 0.8536 & 0.6388 & 0.7544 & 0 & 0.82 \\
\hline
\end{tabular}


Table 4.3. The transaction cost of supplier in previous years (\$ /Kilogram)

\begin{tabular}{|c|c|c|c|c|c|c|c|c|c|c|c|c|c|c|c|}
\hline $\begin{array}{l}\text { Period } \\
\text { supplier }\end{array}$ & 2005 & 2006 & 2007 & 2008 & 2009 & 2010 & 2011 & 2012 & 2013 & 2014 & 2015 & 2016 & 2017 & 2018 & 2019 \\
\hline 1 & 0.3668 & 0.3576 & 0.3588 & 0.3736 & 0.4672 & 0.39 & 0.3688 & 0.4676 & 0.4264 & 0 & 0.4956 & 0 & 0 & 0 & 0 \\
\hline 2 & 0.3376 & 0.3604 & 0.362 & 0.42 & 0.5416 & 0.538 & 0.522 & 0.5032 & 0.4212 & 0.402 & 0.3708 & 0 & 0.2596 & 0.2832 & 0.3188 \\
\hline 3 & 0.2052 & 0.218 & 0.2264 & 0.2236 & 0.31 & 0.2968 & 0.26 & 0.3324 & 0.272 & 0.2628 & 0.2404 & 0.1804 & 0.2352 & 0.2836 & 0.2704 \\
\hline 4 & 0.1884 & 0.2148 & 0.2104 & 0.1724 & 0 & 0.2316 & 0 & 0 & 0.3136 & 0.1408 & 0.208 & 0.1668 & 0.1524 & 0.1256 & 0.2388 \\
\hline 5 & 0.3024 & 0.2884 & 0.3684 & 0 & 0 & 0.5168 & 0.436 & 0 & 0 & 0 & 0 & 0 & 0 & 0.506 & 0.4356 \\
\hline 6 & 0 & 0 & 0 & 0 & 0.438 & 0.254 & 0.3448 & 0.3764 & 0.366 & 0.3576 & 0.27 & 0.1988 & 0.2168 & 0.246 & 0.2824 \\
\hline 7 & 0 & 0 & 0 & 0 & 0 & 0.3076 & 0.3192 & 0 & 0 & 0 & 0.3568 & 0 & 0.4104 & 0.3252 & 0.2956 \\
\hline 8 & 0 & 0 & 0 & 0 & 0 & 0 & 1.221 & 1.301 & 1.176 & 1.048 & 1.024 & 0.767 & 0.905 & 0 & 0.984 \\
\hline
\end{tabular}

Table 4.4. The market price of product in previous years ( $\$ /$ Kilogram)

\begin{tabular}{cccccccccccccccc}
\hline Period & $\mathbf{2 0 0 5}$ & $\mathbf{2 0 0 6}$ & $\mathbf{2 0 0 7}$ & $\mathbf{2 0 0 8}$ & $\mathbf{2 0 0 9}$ & $\mathbf{2 0 1 0}$ & $\mathbf{2 0 1 1}$ & $\mathbf{2 0 1 2}$ & $\mathbf{2 0 1 3}$ & $\mathbf{2 0 1 4}$ & $\mathbf{2 0 1 5}$ & $\mathbf{2 0 1 6}$ & $\mathbf{2 0 1 7}$ & $\mathbf{2 0 1 8}$ & $\mathbf{2 0 1 9}$ \\
\hline Price & 2.94 & 2.94 & 2.62 & 2.393 & 2.938 & 2.78 & 2.176 & 2.59 & 2.3 & 1.87 & 1.66 & 1.536 & 1.6 & 1.408 & 1.344 \\
\hline
\end{tabular}




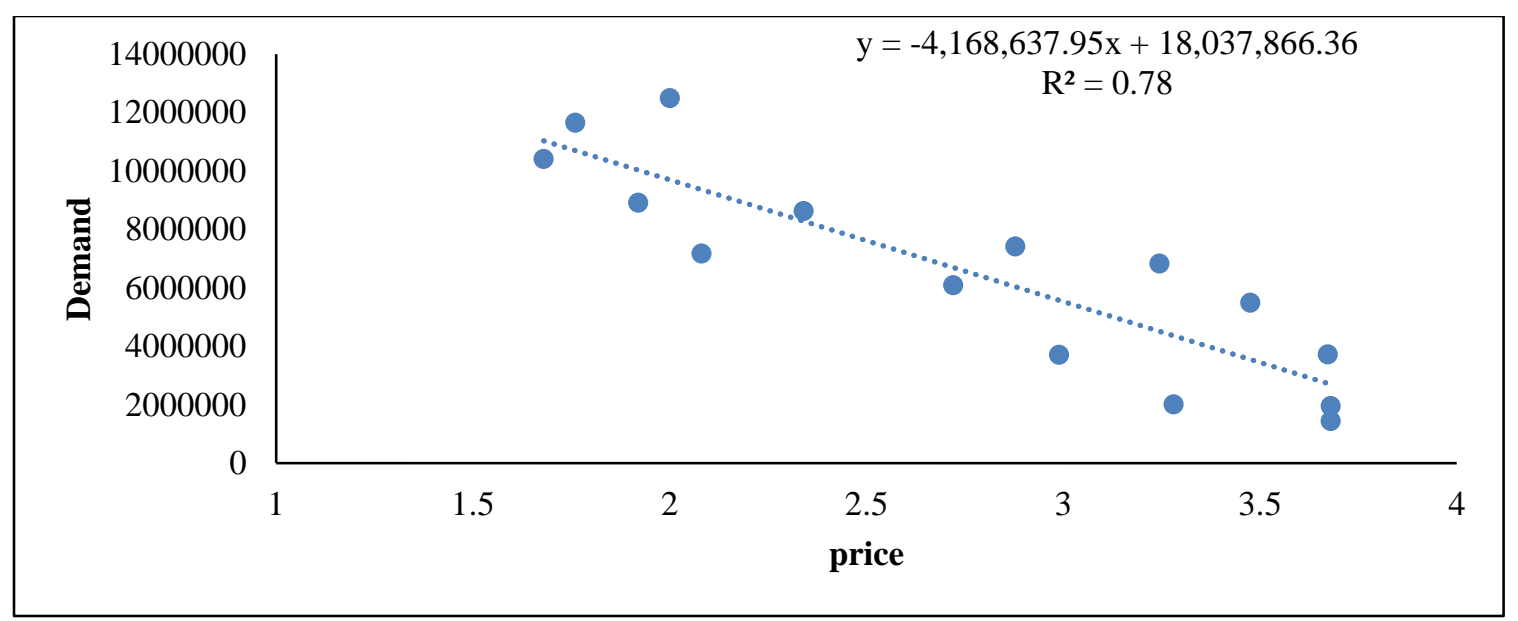

Figure 4.2. The demand function of the product

Table 4.5. The conjecture price of supplier in the previous years $\left(\theta_{i, t} \times 10^{8}\right)$

\begin{tabular}{cccccccccccccccccc}
\hline $\begin{array}{c}\text { Period } \\
\text { supplier }\end{array}$ & $\mathbf{2 0 0 5}$ & $\mathbf{2 0 0 6}$ & $\mathbf{2 0 0 7}$ & $\mathbf{2 0 0 8}$ & $\mathbf{2 0 0 9}$ & $\mathbf{2 0 1 0}$ & $\mathbf{2 0 1 1}$ & $\mathbf{2 0 1 2}$ & $\mathbf{2 0 1 3}$ & $\mathbf{2 0 1 4}$ & $\mathbf{2 0 1 5}$ & $\mathbf{2 0 1 6}$ & $\mathbf{2 0 1 7}$ & $\mathbf{2 0 1 8} \mathbf{2 0 1 9}$ \\
\hline $\mathbf{1}$ & 8.4 & 0.7 & 1.05 & 1.4 & 5.25 & 5.25 & 9.8 & 5.25 & 11.9 & - & 4.2 & - & - & - & - \\
$\mathbf{2}$ & 6.65 & 7 & 4.55 & 4.9 & 14.7 & 12.95 & 2.8 & 10.85 & 15.05 & 3.15 & 5.25 & - & 2.8 & 2.8 & 3.15 \\
$\mathbf{3}$ & 7 & 5.25 & 3.5 & 4.9 & 7 & 6.3 & 12.6 & 7 & 7.35 & 8.4 & 16.8 & 11.55 & 2.8 & 2.8 & 2.8 \\
$\mathbf{4}$ & 7.7 & 8.05 & 4.2 & 10.15 & - & 29.05 & - & - & 22.75 & 18.9 & 8.75 & 5.25 & 2.8 & 3.15 & 3.15 \\
$\mathbf{5}$ & 2.1 & 1.4 & 1.75 & - & - & 21.35 & 32.2 & - & - & - & - & - & - & 2.8 & 3.15 \\
$\mathbf{6}$ & - & - & - & - & 29.05 & 28.7 & 4.55 & 7.35 & 4.2 & 3.15 & 4.9 & 2.45 & 2.1 & 2.1 & 2.1 \\
$\mathbf{7}$ & - & - & - & - & - & 28.7 & 4.55 & - & - & - & 4.2 & - & 2.8 & 2.8 & 3.15 \\
$\mathbf{8}$ & - & - & - & - & - & - & 3.85 & 7 & 4.2 & 1.75 & 11.2 & 5.95 & 2.8 & - & 3.15 \\
\hline
\end{tabular}

Table 4.6. The results of the proposed model for different levels of capacity

\begin{tabular}{|c|c|c|c|c|c|}
\hline $\begin{array}{c}\text { Capacity } \\
\text { level }\end{array}$ & $\begin{array}{c}\text { Cost of } \\
\text { capacity } \\
\text { generation }\end{array}$ & $\begin{array}{c}\text { NPV of } \\
\text { Licence } \\
\text { profit }\end{array}$ & $\begin{array}{c}\text { NPV of } \\
\text { Licensor } \\
\text { profit }\end{array}$ & $\begin{array}{c}\text { NPV of Sales } \\
\text { income } \\
\text { without tax }\end{array}$ & $\begin{array}{c}\text { Partners' gain } \\
\left(\pi_{F}-\pi_{S P, F}\right)\end{array}$ \\
\hline $\mathbf{1 0 0 0 0}$ & 25200000 & 10773000 & 4159739 & 129975597 & 4159739 \\
\hline $\mathbf{9 0 0 0}$ & 22680000 & 10410120 & 4458185 & 139606219 & 4458185 \\
\hline $\mathbf{8 0 0 0}$ & 20160000 & 10901520 & 5610911 & 175127532 & 5610911 \\
\hline $\mathbf{7 0 0 0}$ & 17640000 & 7965783 & 3336500 & 103460572 & 3336500 \\
\hline $\mathbf{6 0 0 0}$ & 15120000 & 6645240 & 2677283 & 80980097 & 2677283 \\
\hline $\mathbf{5 0 0 0}$ & 12600000 & 4726134 & 1419503 & 44122311 & 1419503 \\
\hline $\mathbf{4 0 0 0}$ & 10080000 & 3668112 & 1022808 & 31015799 & 1022808 \\
\hline $\mathbf{3 0 0 0}$ & 7560000 & 2268709 & 284731 & 8869572 & 284731 \\
\hline $\mathbf{2 0 0 0}$ & 5040000 & 1453032 & 130380 & 4121645 & 130380 \\
\hline $\mathbf{1 0 0 0}$ & 2520000 & 670950 & 9624 & 299794 & 9624 \\
\hline
\end{tabular}


Another remarkable note is the worth of the stochastic approach used in the model. If we just use the mean of uncertain parameters and consider the capacity level of 8000 tons, then the sum of the partners' profit, which reflects the value of the alliance, will be equal to $\$ 170454425$, which is less than that of the stochastic approach (180738443). Therefore, the uncertain approach adds $180738443-170454425=\$ 10284018$ to the value of the alliance.

We examine the effects of changes in two parameters of the model, which represent the characteristic of licensors. Doing so, some managerial insights about partner selection could be extracted, especially when the licensee or local government has alternatives to grant them the exclusive right of production. These parameters are the conjecture price and the return on the product of the licensor. In the sensitivity analysis, the change $+a \%$ means that the considered parameter is multiplied by $(1+\mathrm{a} / 100)$ while $-\mathrm{a} \%$ means that the parameter is multiplied by the coefficient (1-a/100).

\subsubsection{Changes in the conjecture price of the licensor $\left(\theta_{L F, t}\right)$}

Figure 4.3 shows the changes in the partners' gain against the conjecture price of the licensor. According to this result, the partners' gain is increased by increasing the conjecture price of the selected licensor. This could be explained by the fact that more values of the conjecture price result in more market power and share for the alliance and consequently more profits for the partners.

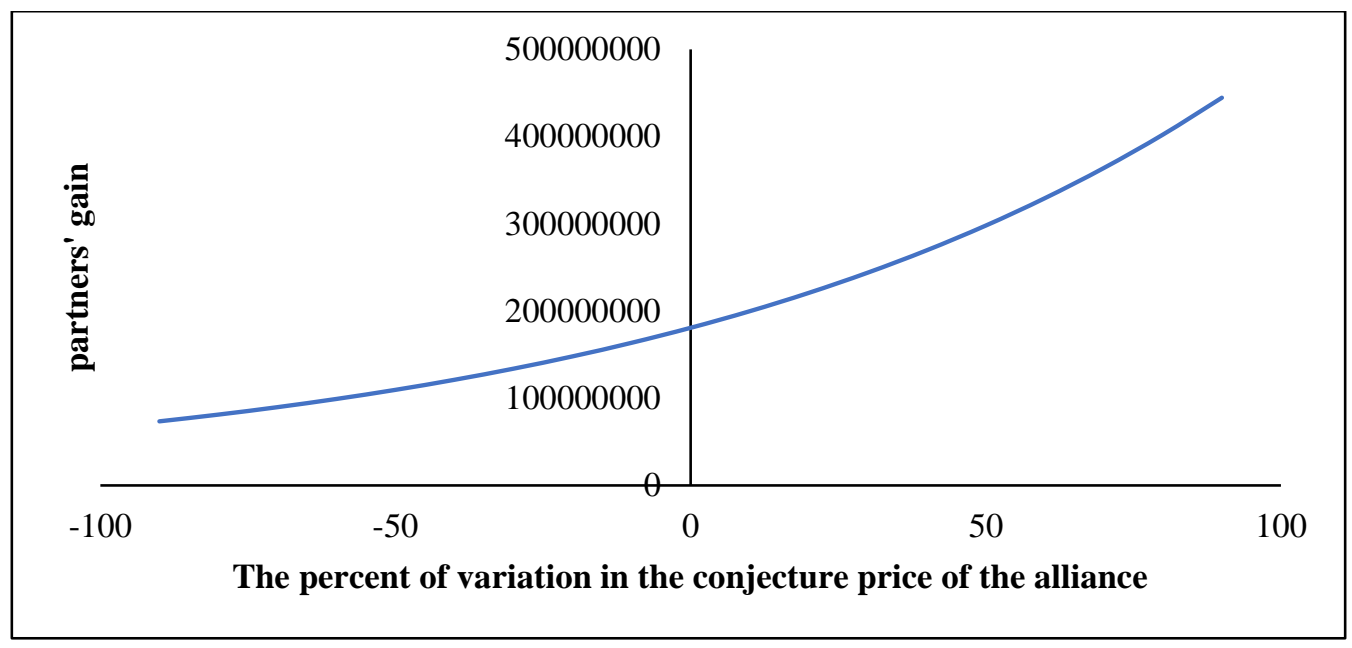

Figure 4.3. The partners' gain versus the conjecture price of the licensor 


\subsubsection{Changes in the return on the capital of the licensor $\left(R O C_{F}\right)$}

In Figure 4.4 the partners' gains have been depicted for different values of $R O C_{F}$. Based on the results, as long as $R O C_{F}$ is more than 0.25 , the licensee ownership is optimal, and due to the lack of licensor investment, there are no changes in the partners' gain. However, for the value of $R_{O C} F$ less than 0.25 , the shared ownership is optimal, and, in such cases, the higher the $R O C_{F}$, the more stand-alone profit of the licensor will be, and hence the synergy from the partnership will be decreased. These results are valuable in two directions: First, among the licensors, the one which finds more profit in the other market or its own business than the local market is preferable. However, if there is no such licensor, then the one that has less $R O C_{F}$ will request fewer values for the usage of their patent right and provide more profit for the local firm referred to as the licensee.

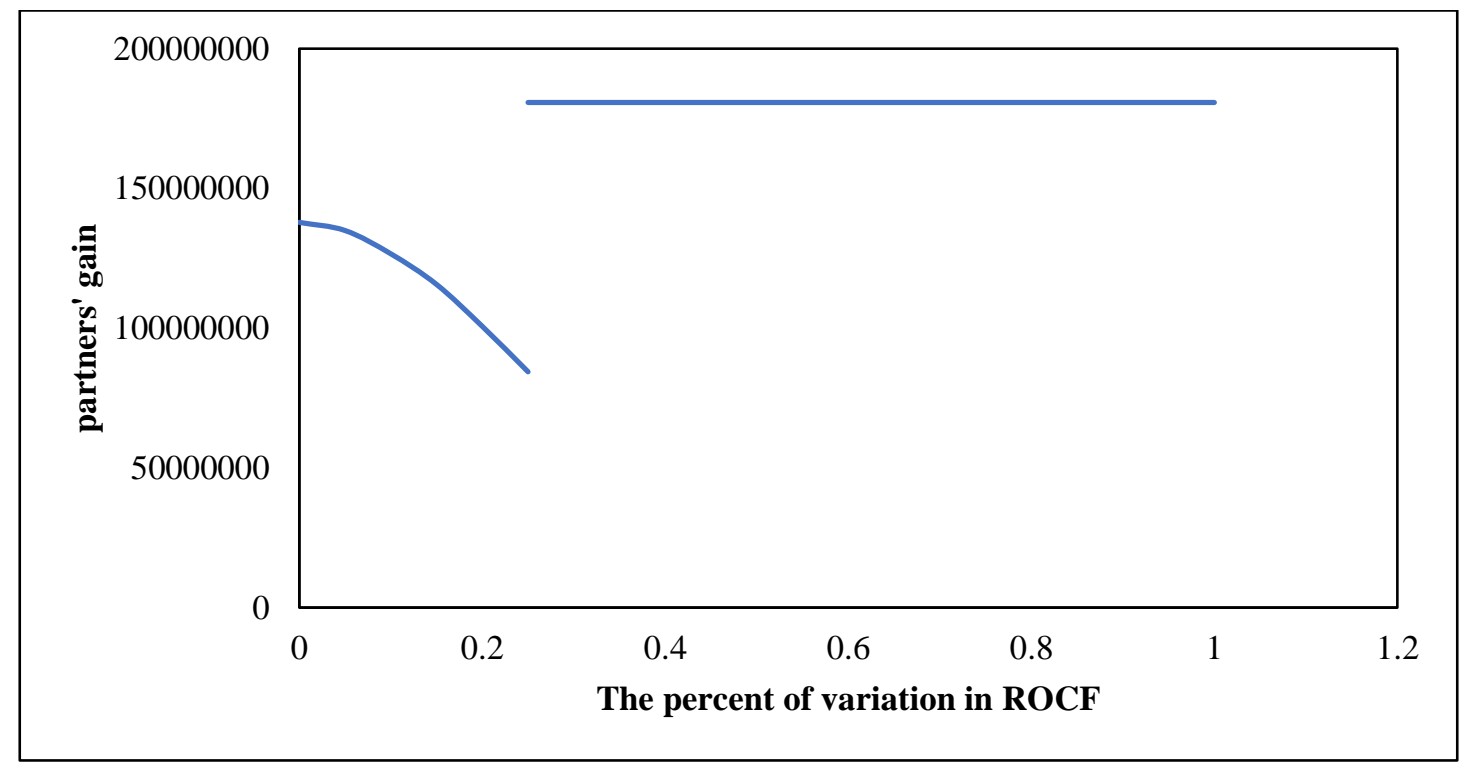

Fig 4.4. The partners' gain versus the return on the capital of the licensor

\subsection{Conclusion}

In this chapter, a new model has been proposed for taking into account the value of cooperation when a local pharmaceutical manufacturer decides to obtain the production right of a patented product from its main manufacturer. The proposed model determined the assigned profit of members by considering various aspects of partners and market, including the oligopoly structure of the market for homogenous products, the effects of partnership on the reduction in the 
transaction cost, the financing sources of the alliance, the possibility of liquidation when the alliance is evaluated to be non-profitable in future and the uncertainty in the model parameters. To tackle the uncertainty, the stochastic programming approach was utilized, and the scenarios of the uncertain parameters were generated in a way that the known market properties such as path dependency and market shocks were taken into account. Also, due to the complexity of the model, an innovative dynamic programming approach was proposed to optimize the partners' gain by determining the parameters of the license contract and the ownership share.

The proposed model was implemented for valuing an alliance to produce Citric Acid as the main component of many pharmaceutical products under a license contract in Iran. The results showed that the proposed stochastic approach regards more value for the alliance than a deterministic approach, which represents the uncertain parameters with their mean value. Moreover, the reached royalty rate of the model was illustrated to be in the common range of the reported royalty rates in pharmaceutical products. Furthermore, the sensitivity analysis was carried out to examine the effects of the licensor specification on the gain of cooperation. The examined parameters were the conjecture price and the return on the capital of the licensor. The results indicated that the conjecture price of the licensor had a positive effect on the gain of cooperation, while the return on the capital of the licensor had adverse effects on the gain if the shared ownership was optimal and had no effect on the gain otherwise. The managerial insights of such an investigation were explained as follows:

- If the local manufacturer or the government is able to choose a licensor among several alternatives, the one with the maximum conjecture price is the best choice. Such a high conjecture price is the result of more market share and power of the related licensor and ensures a high amount of selling and selling profit for the alliance.

- The licensors who find more benefit in their own business or have a high enough return on capital are preferred licensors. On the other hand, if the licensor's return on the capital rate be such that the shared ownership of the alliance is optimal, the licensors with less rate will provide more profit for the licensee. Such licensors are beneficial because they will claim less stand-alone profit. 


\section{Chapter 5: A new pricing mechanism for pharmaceutical supply chain: A cooperative game theory approach}

Due to the significant role of health care services in saving human lives, the value of health services for customers is very high, and with a lack of appropriate control, the supply price of such services can run to a great amount. Therefore, the government oversees a significant portion of health care costs and enacts special controls on the pricing of health care services. The government is also always seeking ways to reduce health care expenditure. In this chapter, exclusive domestic production of licensed brand medicines by the formation of a cooperative firm between a domestic manufacturer and a foreign licensor is studied as a cooperative strategy that could reduce the franchise related costs for the government. This strategy could provide many advantages for the government and the manufacturers. One advantage, the increase in the income of the manufacturer, is studied in this chapter. Because of this benefit, the government also expects discounts from the cooperative firm, and the value of the discount depends on the game between the government and the firm. In this regard, the Nash bargaining solution of the cooperative game theory is proposed to model the related pricing negotiations between the partners. The proposed model is applied in a real case study, and a set of sensitivity analyses is carried out to show how different situations affect the pricing decision and the distribution of the resulting surplus between partners. The cooperative strategy results indicate that the market share of the licensor on the local market and the return on the capital (ROC) of the local licensee manufacturers positively affect the equilibrium price of the model and have negative impacts on the likelihood of forming a cooperative firm.

\subsection{Introduction}

The supply chain paradigm has changed the individual behavior of organizations toward a cooperative and coordinated one. The supply chain managers consider all operations of the members and make decisions so that the performance of the supply chain is optimized. This does not mean that all members will benefit from the integrated decisions of the supply chain. Therefore, some mechanisms are needed to provide enough incentives for the members by allocating them an appropriate amount of the supply chain profit. This allocation is such that the income of members after the allocation is at least equal to their income if they had operated independently. The integration and cooperation of the supply chain have been the main subjects of supply chain 
research in recent years, and in the present chapter, the same subject is investigated in the context of the pharmaceutical supply chain.

Due to the importance of the health system, governments in almost all countries, regardless of their income level, pay considerable attention to this sector of society and pay for most of the medical costs through public insurances or other reimbursement schemes (Vogler and Martikainen, 2015). Regarding the importance and structure of the pharmaceutical supply chain and the role of government in affording a significant part of costs in PSC, we present the following motivations in this chapter:

Motivation 1: Such trends reveal the desire of the government to control and reduce the health system cost. Governments could establish an alliance with other players of the PSC and benefit from the resulting synergy of the cooperation. In doing so, the government could grant the rights and permissions of exclusive products to the producers and distributors of PSC, and other members could help the government by supplying the medicines at a lower price than the common market price.

Motivation 2: All the PSC members, including the government, could improve their performance by engaging in such a strategy. Considering such a viewpoint about the cooperation between the PSC members, we study the pricing problem of expensive medicines using the cooperative game theory. The pricing scheme, as explained later will be such that the profit for the government by way of reducing medicine cost per patient is balanced against the profit made by the producers for applying noteworthy amounts of the exclusive medicine in the local market.

Also, notably, the proposed methodology is not a mere pricing method, but it just modifies the current market price in a way that the competitive advantages of partners could be taken into account. It could be regarded as a discounting scheme which provides adequate motivations for partners to cooperate with each other. Therefore, the mechanism is not to be claimed to be in competition with another pricing method, and it does not neglect the remarkable hypothesizes hidden in the represented price of medicines. The possibility of using a discount to offer lower prices has been mentioned in other papers such as Csanádi (2018).

Motivation 3: Another important subject when one considers the possibility of the domestic production of brand medicines is the value of the license. Granting the brand medicines' formulas 
entails a special payment to the secondary manufacturer of the medicines as they own the formulas and spent lots of effort and time to develop them. Such payment usually is arranged in the form of a license contract. A license contract creates the need for another coalition between the domestic manufacturer and the secondary manufacturer. This extends the framework of the game from one coalition to two coalitions. The first coalition is established between the domestic and the secondary manufacturers to determine the value of the license contract parameters and to form the cooperative firm. On the other hand, in the second coalition, the government and the cooperative firm negotiate the new price of the medicines after granting the exclusive right of production by the government to the cooperative firm.

Motivation 4: Although the problem of each coalition is discussed separately between the related partners of each coalition, those problems are not distinct, and as shown, they should be studied in an integrated and dependent framework. This kind of analysis is the novelty of the current study, which besides the embedded logic of the cooperative benefits and the requirement for presenting a discounted price, provides an integrated and supportive framework for decision-making and addressing the pricing and license valuation problem in the regarded alliance.

In the next sections of the chapter, the models of the problem and the results of implementing the study on a real numerical example are presented.

\subsection{The proposed model}

In our proposed model, we assume that a domestic company is negotiating with a brand-name medicine manufacturer over a license and is establishing a domestic company. Given the incentives provided by the local government for such an investment, the establishment of this company can have significant benefits to the partners, and the license fee can be modeled using cooperative game theory and by considering the benefits of the project. Before describing the model in detail, the model assumptions are presented as follows:

Assumption 1: Given the fact that the process of registering medicines in the local market and obtaining the needed licenses is time-consuming, it is presumed that the selected brand-name medicine is a well-recognized product in the local market. 
Assumption 2: The pricing process of medicines varies around the world, and it is determined by the policies posed by local governments. In the present study, the pricing mechanism that specifies the sales revenue of a company is determined by the local pricing process.

Assumption 3: Although there are several operational benefits for establishing a licensed company in the domestic market, the present study merely considers the benefits of this program in reducing transaction costs (tariffs and other import costs) and increasing market share for partners. Other benefits, such as having better production plans rather than importing products and the availability of sufficient medicines, are left untouched, and future studies need to account for such issues.

Assumption 4: In the present study, the planning horizon is considered equal to the remaining lifespan of the patent. In fact, after this time horizon, the formulation of the medicine is considered to be generic, and no licensing right is preserved for the company. Also, we measure the company's final value in the time horizon equal to the value of a generic company and by considering the remaining life of the equipment.

Assumption 5: As was mentioned, local governments usually facilitate the cooperation of foreign companies with domestic ones to support Gross Domestic Product (GDP). Some measures include tariff cuts for importing pharmaceutical raw materials and canceling the importation of other brands when there is domestic production.

Considering the above assumptions, various aspects of the problem are discussed in the following subsections, and then the integrated model of the chapter is presented.

\subsubsection{Medicine pricing and demand}

Taking into account the pricing mechanism in the literature review, the reference-based pricing mechanism is investigated in this chapter which is the current pricing mechanism in the country under study, namely, Iran.

According to the mentioned pricing mechanism of the country, the prices of brand name medicines are compared among reference countries, including Australia, Greece, Spain, Portugal, Turkey, and the medicine manufacturer country itself, then, the lowest price available in these countries is considered as the base rate for pricing. After importing the medicines, customs tariffs and regular 
taxes are added to the reference price (20-35\% for brand name medicines), and then a profit margin of up to $15 \%$ is considered for the importer. The profit margin of pharmacies for domestic products is $20-26 \%$, and for products used in hospitals or imported medicines, the margin is $15-20 \%$. Moreover, the profit margin of distributors is $50 \%$ of the profit margin of pharmacies, and this rate can be increased by up to $20 \%$ for cold chain medicines and narcotic analgesics. It should also be mentioned that if a domestic company, under a license agreement with a foreign company, attempts to produce a domestic brand name medicine, the price is set at a certain percentage of the medicine import price by a medicine pricing commission sets that price.

Most governments and insurance companies prefer generic medicines to brand-name ones. Since insurance companies usually refuse to cover brand-name medicines, the demand for these medicines is currently at the lowest level possible. However, if some brand-name medicines have been prescribed for chronic and special diseases (e.g., Rebif for MS patients), some support organizations might allocate funds for these medicines and increase the affordability of these medicines for patients.

Since the pricing of medicines is a non-competitive process determined by government organizations, the impact of discounts, induced by price reductions on the company's demand and profitability can be examined. In the present study, we will investigate the latter case and explain the role of governments in improving the outcomes for the patients. According to government regulations, in the case of domestic production of medicine that is related to a special disease, the medicine is financially supported by the government with a low percent franchise. In summary, the pricing process of brand-name medicines is a game between the government, insurance companies, and pharmaceutical companies. In this study, an attempt will be made to discuss various dimensions of this issue by presenting the problem in a specific case.

\subsubsection{The collaborative profit of parties}

As explained, creating a licensed company to produce a brand-name medicine, at first, involves a coalition consisting of a foreign company with a medicine patent and a domestic company as an investor. The local government, as the third player outside the alliance of these two companies, acts as a financial facilitator by paying the discrepancy between medicine prices, and franchise 
received from patients. Also, the local government plays a direct role in determining the price of the medicine, and it can provide incentives for both parties to participate more actively especially foreign companies. If we consider that a newly established company is responsible for supplying the demand, we believe the capacity of the establishing company should be equal to its annual demand in the country.

Assuming that the company's sales price to suppliers is equal to $p_{C}$ and the annual demand is equal to $q$, then the domestic company's sales revenue will be equal to $p_{c . q}$. Taking into account production cost is equal to $c_{L}$ and the annual depreciation of the company is equal to $D P_{L}$, the operating profit excluding deductions of tax and license fee can be shown using Eq. (5.1):

$$
\pi=\left(p_{C}-c_{L}\right) \cdot q-D P_{L}
$$

Furthermore, the foreign company grants permission to the domestic company through a license contract to use its patented product. A common form of the licensing contract is determined by considering a fixed amount of lump-sum amount $(L)$ and a royalty percentage relative to sales $(r)$. According to the contract, the payoff of the licensor $(L P)$ can be shown by Eq. (5.2) (Vishwasrao, 2007; Sercu, 2008):

$$
L P=L+r \cdot p_{C} \cdot q
$$

Now, considering the tax rate on the sales of the alliance is equal to $\tau_{L}$ and denoting the tax rate on the revenue of licensor by $\tau_{F, 1}$, the revenue functions of the licensee $\left(\pi_{L, C}\right)$ and licensor $\left(\pi_{F, C}\right.$ ) in the cooperative strategy are expressed by using Eqs. (5.3) and (5.4):

$$
\begin{aligned}
& \pi_{L, C}=\left(1-\tau_{L}\right)\left(\left(p_{C}-c_{L}\right) \cdot q-D P_{L}-L-r \cdot p_{C} \cdot q\right) \\
& \pi_{F, C}=\left(1-\tau_{F, 1}\right) \cdot L P=\left(1-\tau_{F, 1}\right) \cdot\left(L+r \cdot q \cdot p_{C}\right)
\end{aligned}
$$

To determine the revenue function of the government as the third player after establishing the cooperative strategy, by government regulations, we assume that the medicine under consideration has $x$ percent of the franchise paid by the consumers, and the rest of this money is paid by the government. Considering the profit margin of pharmacies as ps and the profit margin of distributors as $d s$ percent of the profit margin of pharmacies, we can calculate the revenue function of the government as described in Eq. (5.5). 


$$
\pi_{G, C}=-(1-x) \cdot(1+p s .(1+d s)) \cdot p_{C} \cdot q
$$

It should be noted that in Eq. (5.5), government revenue has been shown with a minus sign because the government only covers the cost of patients' medicine using the tax received or other revenue. More precisely, the term $(1+p s .(1+d s)) \cdot p_{C}$ represents the price of the medicine in the patients' prescriptions after adding the profit margins of distributors and pharmacies to the manufacturer's sales price. It is worth mentioning that in the health care system under study, a distributor's profit margin is calculated as $d s$ portions of the profit margins of distributors. This means that the profit margins of pharmacy; $p s . p_{C}$ and the profit margins of distributors; $d s . p s . p_{c}$ are added to the reference price $p_{c}$ to account for the price in the prescriptions of patients i.e., $(1+p s .(1+d s)) \cdot p_{C}$. Therefore, $(1+p s .(1+d s)) \cdot p_{C} \cdot q$ denotes the total cost of patients, $(1-\mathrm{x}) \times 100$ percent of which should be paid by the government.

\subsubsection{The profit of parties before collaboration}

In this study, cooperative game theory is used to decide on different issues such as sales prices of medicines, determining the licensing contract parameters, and the revenues generated by the partnership. We first outline the revenue of the partners before the cooperation.

As explained in the pricing section, the price of importing brand-name medicines is equal to their lowest price in the reference countries. In this case, we assume that the price of a brand-name medicine before establishing a local company is $p_{B}$. With this description, the revenue of the government before creating a partnership is presented as Eq. (5.6):

$$
\pi_{G, N}=-(1-x) \cdot(1+p s \cdot(1+d s)) \cdot p_{B} \cdot q
$$

Moreover, if the domestic company does not engage in the cooperative alliance, it can save the expenses of annual depreciation costs. The ROC (Return on Capital) index is one of the most important indicators that can be used to demonstrate the capability of a company to generate a return on its investment. This indicator shows the company's profit after tax deduction in proportion to the total assets of the company, including equity and the company's debts. Assuming the existence of financial transparency in the accounting documents, this indicator can easily be calculated and agreed upon by other partners. Therefore, considering the incurred annual 
depreciation of the new firm as the investment of the domestic company, the predicted profit of the domestic company before participation can be calculated by Eq. (5.7) (Dewandaru, 2015).

$$
\pi_{L, N}=\left(1+R O C_{L}\right) \cdot D P_{L}
$$

It is also assumed that the foreign company's stock market share in the country under study is $s$ percent of the total demand of the market before cooperation. The company will sell the products at the price of $P_{B}$ to the importers; considering the variable of production cost as $V_{F}$, depreciation

of the local market demand as $D P_{F}$, foreign company's income tax as $\tau_{F, 2}$ (the tax on the operational income not on the license income), and the per-unit operation cost of the medicine as $c_{F}$, the profit of this company before participation $\left(\pi_{F, N}\right)$ is determined using Eq. (5.8). This profit consists of the sales income minus the production and depreciation costs taxed at the proper rate.

$$
\pi_{F, N}=\left(1-\tau_{F, 2}\right)\left(\left(p_{B}-c_{F}\right) . s . q-D P_{F}\right)
$$

\subsubsection{The joint venture bargaining model}

In the present study, the Nash bargaining model is used to model market price modification, partners' revenue, and analysis of different situations governing the problem. We have two coalitions, the first is the partnership of the domestic company as an investor with the foreign company which owns the patent, and the second one is the coalition between the newly established company and the government. In each coalition, we can use the Nash bargaining solution to specify the outcomes of members.

Nash bargaining model was presented as one of the most widely used models of cooperative games in the 1960s. Nash bargaining solution has two classical axioms: individual rationality and Pareto optimality. John Nash added three other axioms, including symmetry, linear invariance, and independence of irrelevant alternatives, and showed that a bargaining game could have a unique solution as the core of the game. The first axiom states that partners are not willing to participate in the coalition if they get higher payoffs outside the coalition. The second axiom, Pareto optimality, claims that if there is another solution through which at least one of the partners gets higher payoffs compared to the existing solution, then the players will not accept the existing solution. While symmetry suggests the symmetric equilibrium of the available solutions, linear 
invariance states that any linear transformation on the utility function of one player should not change the optimal bargaining solution. In fact, after this transformation, the payoff of the player is equal to the player's revenue image before this transformation. Finally, the independence of irrelevant alternatives indicates that the equilibrium of the bargaining game does not change if the non-equilibrium responses are eliminated from the space available of a bargaining game solution, and the optimal response and disagreement payoff $(d)$ is maintained. Considering these axioms, the Nash bargaining solution in a two-player game can be determined using the mathematical programming model (5.9):

$$
\begin{array}{ll}
\max \left(\pi_{1}-t_{1}\right) \cdot\left(\pi_{2}-t_{2}\right) \\
\text { s.t. } \\
\qquad \begin{array}{l}
\pi_{1}+\pi_{2}=\Pi \\
\pi_{1} \geq t_{1} \\
\\
\pi_{2} \geq t_{2}
\end{array}
\end{array}
$$

In model (5.9), $t_{1}$ and $t_{2}$ represent the stand-alone payoff of players 1 and 2, respectively, $\Pi$ represents the payoff generated by the formation of the coalition by the two players, and $\pi_{1}, \pi_{2}$ are the payoffs generated by Nash bargaining solution for players 1 and 2 respectively. Obviously, for model constraints, we need to have $\Pi \geq t_{1}+t_{2}$; otherwise, the cooperation of the two players and the formation of a coalition for individual rationality axiom will not be meaningful.

Considering the above conditions, the objective function of the above-mentioned mathematical programming problem is a concave function. Without concerning the constraints $\pi_{1} \geq t_{1}$, and $\pi_{2} \geq t_{2}$ and just by applying the constraint $\pi_{1}+\pi_{2}=\Pi$, the optimal solution of the Nash bargaining problem is obtained by Eqs. (5.10) and (5.11):

$$
\begin{aligned}
& \pi_{1}=\frac{\Pi+t_{1}-t_{2}}{2} \\
& \pi_{2}=\frac{\Pi-t_{1}+t_{2}}{2}
\end{aligned}
$$

Considering $\Pi \geq t_{1}+t_{2}$, we can clearly conclude that for $\pi_{1}$ and $\pi_{2}$ in Eqs. (5.10) and (5.11), the relations $\pi_{1} \geq t_{1}$ and $\pi_{2} \geq t_{2}$ hold. For example, in this case, we have: 
$\pi_{1}=\frac{\Pi+t_{1}-t_{2}}{2} \geq \frac{t_{1}+t_{2}+t_{1}-t_{2}}{2}=t_{1}$. Furthermore, if $\Pi<t_{1}+t_{2}$, then it is easy to prove that in the bargaining solution, the constraints of the model (5.9) will not be established and that the model will not have a feasible answer. Therefore, the necessary and sufficient condition for having a costeffective construction of a company and for Eqs. (5.10) and (5.11) to be the answer of the bargaining model is that $\Pi \geq t_{1}+t_{2}$. Given this equation, the minimum market price at which partners would be willing to participate and establish the company could be obtained. So, Lemma 5.1 is described as follows:

Lemma 5.1: The necessary and sufficient condition for achieving the Nash bargaining solution in a two-player game is $\Pi \geq t_{1}+t_{2}$. If this condition is established, the payoffs generated by the formation of a coalition for players 1 and 2 are obtained using Eqs. (5.10) and (5.11), respectively. In this case, through considering Eqs. (5.3) and (5.4), the payoff of the coalition between domestic and foreign companies $\left(\Pi_{L F}\right)$ is obtained by $\Pi=\Pi_{L, F}=\pi_{L, C}+\pi_{F, C}$. Also, given $\pi_{1}=\pi_{L, C}$, $\pi_{2}=\pi_{F, C}, t_{1}=\pi_{L . N}, t_{2}=\pi_{F . N}$, and Eq. (5.10), the licensing revenue is determined using Eq. 5.12:

$$
\begin{aligned}
& L P=L+r \cdot p_{C} \cdot q= \\
& \frac{\left(\left(p_{C}-c_{L}\right) \cdot q-D P_{L}\right) \cdot\left(1-\tau_{L}\right)-D P_{L} \cdot(R O C+1)}{2-\tau_{L}-\tau_{F, 1}}+\frac{\left(q . s \cdot\left(p_{B}-c_{F}\right)-D P_{F}\right) \cdot\left(1-\tau_{F, 2}\right)}{2-\tau_{L}-\tau_{F, 1}}
\end{aligned}
$$

Any positive integer for $r$ and $L$ that balances Eq. (5.12) can be parameters of the licensing contract. Considering the licensing revenue in Eq. (5.12), the payoff functions of the domestic and foreign companies in the cooperative strategy, i.e., $\pi_{L, C}$ and $\pi_{F, C}$ respectively, and the payoff function of the coalition $\left(\Pi_{L F}\right)$ can be rewritten as Eqs. (5.13), (5.14), and (5.15):

$$
\begin{aligned}
\pi_{L, C}= & \left(1-\tau_{L}\right) \cdot\left(\left(p_{C}-c_{L} \cdot q\right) \cdot \mathrm{q}-D P_{L}-\frac{\left(q \cdot s \cdot\left(p_{B}-c_{F}\right)-D P_{F}\right) \cdot\left(1-\tau_{F, 2}\right)}{2-\tau_{L}-\tau_{F, 1}}\right. \\
& \left.-\frac{\left(D P_{L}+q \cdot\left(c_{L}-p_{C}\right)\right) \cdot\left(1-\tau_{L}\right)-D P_{L} \cdot(R O C+1)}{2-\tau_{L}-\tau_{F, 1}}\right)
\end{aligned}
$$




$$
\begin{aligned}
\pi_{F, C}= & \left(1-\tau_{F, 1}\right) \cdot\left(\frac{\left(q \cdot\left(p_{C}-c_{L}\right)-D P_{L}\right) \cdot\left(1-\tau_{L}\right)-D P_{L} \cdot(R O C+1)}{2-\tau_{L}-\tau_{F, 1}}\right. \\
& \left.+\frac{\left(q . s .\left(p_{B}-c_{F}\right)-D P_{F}\right) \cdot\left(1-\tau_{F, 2}\right)}{2-\tau_{L}-\tau_{F, 1}}\right) \\
\Pi_{L F}= & \pi_{L, C}+\pi_{F, \mathrm{C}}
\end{aligned}
$$

Summing up the right-hand side of Eq. (5.13) and Eq. (5.14), we get $\Pi_{L F}$ in Eq. (5.15) as $\Pi_{L F}=\kappa \cdot p_{c}+$ Constant where $\kappa$ is the coefficient of $p_{c}$ in $\Pi_{L F}$.

Given Eqs. (5.13), (5.14) and (5.15), $\kappa$ is expressed as (5.16), which is obviously a positive value. Hence, regarding the positivity of $\kappa$ and taking into account Eqs. (5.7), (5.8), (5.13), (5.14) and (5.15), the minimum market price $\left(p_{c}^{\mathrm{min}}\right)$ in which the partners are willing to form a joint venture is obtained by setting $\Pi_{L F}$ in Eq. (15) equal and greater than $t_{1}+t_{2}$ which results in $p_{c}^{\min }$ according to Eq. (5.17). This price could be regarded as a threshold for the cost-effectiveness of the alliance. Subsequently, if $p_{c} \geq p_{c}^{\mathrm{min}}$ and the relation $\Pi_{L F} \geq t_{1}+t_{2}$ is correct, the establishment of the company will be cost-effective, and both parties will be eager to form it.

$$
\begin{aligned}
& \kappa=\frac{2 q\left(1-\tau_{F, 1}\right)\left(1-\tau_{L}\right)}{2-\tau_{L}-\tau_{F, 1}} \\
& p_{c}^{\min }=\frac{\left(1-\tau_{F 2}\right)}{q \cdot\left(1-\tau_{F 1}\right)}\left(s \cdot q \cdot p_{B}-\left(D P_{F}+c_{F} \cdot q \cdot s\right)\right)+\frac{1}{q}\left(D P_{L}+c_{L} \cdot q+\frac{D P_{L} \cdot(1+R O C)}{\left(1-\tau_{L}\right)}\right)
\end{aligned}
$$

The Eq. (5.17) can be interpreted as follows. The phrase $\frac{\left(1-\tau_{F 2}\right)\left(s . q . p_{B}-\left(D P_{F}+c_{F} \cdot q . s\right)\right.}{q}$ indicates the profit margin of the foreign company after-tax deduction. The price of the product should be such that by balancing the tax effect, at least such a profit margin is provided to the foreign company. The phrase $\frac{D P_{L}+c_{L} \cdot q}{q}$ indicates the net cost per unit of product. Therefore $p_{c}^{\min }$ should be such that covers the unit production cost, the marginal profit of the licensor and $\frac{D P_{L} \cdot(1+R O C)}{q .\left(1-\tau_{L}\right)}$ i.e., the investment cost per unit of product afforded by the licensee regarding its rate of return on capital. Therefore, Proposition 5.1 is expressed as follows: 
Proposition 5.1: The necessary and sufficient condition for the foreign and domestic companies to form a strategic alliance for manufacturing medicines in the domestic company's country is to obtain the minimum selling price of the medicine in the domestic market by Eq. (5.15).

Proposition 5.1 does not take into account the role of the government in facilitating the formation of the alliance. To complete the proposition in deriving the necessary and sufficient condition for the affordability of the newly established company in the local country, Proposition 5.2 is expressed as the following:

Proposition 5.2: The necessary and sufficient condition for the formation of a domestic medicine manufacturing company with the permission of the licensor is that the minimum price acceptable to the coalition consisting of foreign and domestic companies $\left(p_{c}^{\text {min }}\right)$ should be lower than $p_{B}$ in Eq. (5.17).

To prove Proposition 5.2, the following explanations are considered. The newly established company could negotiate with the government to determine the market price. Again, we use the Nash bargaining model to determine the Nash equilibrium price. In this case, $p_{C} \geq p_{C}^{\min }$ and $\pi_{G, C} \geq \pi_{G, N}$ constraints should be implemented to establish the axiom of individual rationality in the established company and the government respectively. Moreover, to make this condition true, the constraint $p_{C} \leq p_{B}$ is necessary, because otherwise, the government has no incentive to grant the necessary permissions and exclusive production rights to the alliance. Therefore, if $p_{c}^{\min }>p_{B}$ , then, the relations $p_{C} \leq p_{B}$ and $p_{C} \geq p_{C}^{\mathrm{min}}$ never could be held simultaneously, and Proposition (5.1) is not satisfied. On the other hand, regarding the objective function of the Nash bargaining solution, the optimal solution should hold the relation $p_{c}^{\min } \leq p_{c} \leq p_{B}$. Because the values of $p_{c}$ outside the interval $\left[p_{c}^{\min }, p_{B}\right]$ make the objective function of Nash bargaining model; $\left(\pi_{L, C}+\pi_{F, C}-\pi_{L, N}+\pi_{F, N}\right) \cdot\left(\pi_{G, C}-\pi_{G, N}\right) ;$ negative, while inside the interval, the objective function is zero or positive, as shown in Figure (5.1). Therefore, when $p_{c}^{\min } \leq p_{B}$, the solution based on Eqs. (5.10) and (5.11) not only ensures the constraints of the Nash bargaining solution, but also it is proved that is placed in the interval $\left[p_{c}^{\min }, p_{B}\right]$. 
The following settings is considered for using Eqs. (5.11) and (5.12) to find the equilibrium price when the necessary and sufficient condition $p_{c}^{\min } \leq p_{B}$ is established:

- $\pi_{1}=\pi_{G, C}, \pi_{2}=\pi_{L, C}+\pi_{F, C}, \quad \Pi=\Pi_{G L F}=\pi_{G, \mathrm{C}}+\Pi_{L F}=\pi_{G, \mathrm{C}}+\pi_{L, \mathrm{C}} \quad$ regarding Eqs. (5.3), (5.4) and (5.5).

- $t_{1}=\pi_{G, N}$ regarding Eq. (5.6).

- $t_{2}=\pi_{L, N}+\pi_{F, C}$ regarding Eqs. (5.7) and (5.8).

Doing so and solving Eqs. (5.11) and (5.12), the equilibrium price in the cooperative strategy will be as Eq. (5.18).

$$
p_{C}^{E}=-\frac{D+E+F}{G+H}
$$

where

$$
\begin{aligned}
& D=-\frac{1}{2} \pi_{G, N}+\frac{1}{2}\left(\pi_{L, N}+\pi_{F, N}\right)+\frac{1}{2}\left(1-\tau_{L}\right) \cdot\left(D P_{L}+c_{L} q\right) \\
& E=\frac{\left(1-\tau_{F, 1}\right) \cdot D P_{L} \cdot(R O C+1)}{2\left(2-\tau_{L}-\tau_{F, 1}\right)} \\
& F=-\frac{\left(\tau_{L}-\tau_{F, 1}\right) \cdot\left(\left(\tau_{L}-1\right) \cdot\left(D P_{L}+c_{L} q\right)+\left(D P_{F}+q \cdot s \cdot\left(c_{F}-p_{B}\right)\right) \cdot\left(\tau_{F, 2}-1\right)\right)}{2\left(2-\tau_{L}-\tau_{F, 1}\right)} \\
& G=-\frac{q \cdot\left(1-\tau_{F, 1}\right) \cdot\left(1-\tau_{L}\right)}{2-\tau_{L}-\tau_{F, 1}} \\
& H=\frac{1}{2} q \cdot(x-1) \cdot(p s \cdot(d s+1)+1)
\end{aligned}
$$




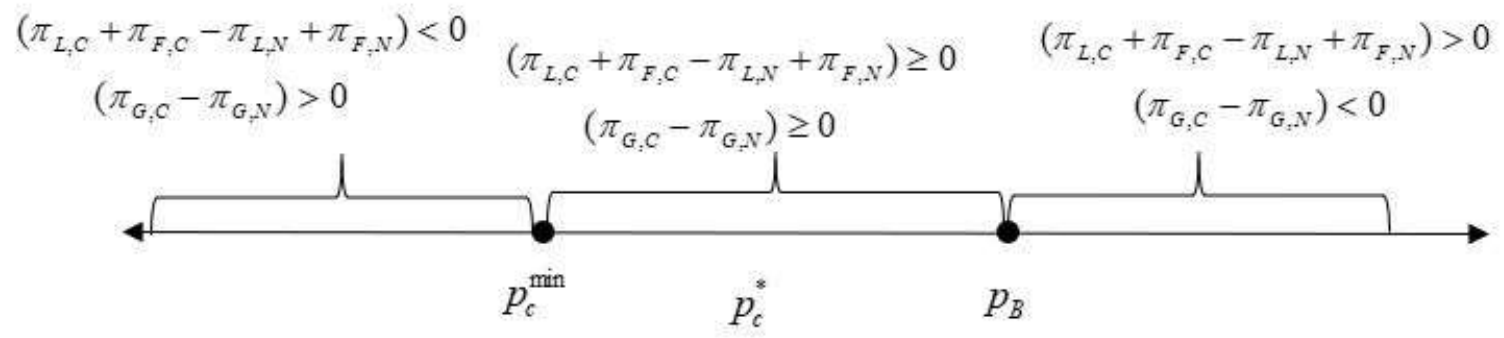

Figure 5.1. The position of the optimal solution of the Nash bargaining model between the government and manufacturer in the case of $p_{c}^{\min } \leq p_{B}$.

\subsection{Case study}

In this section, we implement the proposed methodology for pricing a licensed medicine for the treatment of thalassemia patients in Iran. Thalassemia is caused by low levels of alpha and beta proteins in red blood cells. Hemoglobin is the component of red blood cells that carries oxygen throughout the body and produces alpha and beta proteins. In thalassemia patients, hemoglobin is not able to produce these proteins sufficiently. The patients are categorized into several classes based on the deficiency of each protein and its severity. Thalassemia is not fully curable, and the prescribed medicine only reduces the symptoms and prevents the progression of the disease. Therefore, these patients have to deal with the disease throughout their lives. The best treatment for this disease is blood transfusions, but the adverse effect of this treatment is a transfusioninduced iron overload in the patient's body (since there is no way to remove iron from the body), as a result, thalassemia patients must take medicines to reduce the iron levels in their blood. Jadenu ${ }^{\circledR}$ (deferasirox) is one of the newly developed medicines for this purpose and is produced under the license of Novartis. Strict and rigorous quality control tests in line with FDA standards, including stability tests, have confirmed that Jadenu® performs better than existing medicines such as Exjude®. Therefore, Iran's FDA has granted the exclusive license to manufacture and supply the medicines in the country, and a local company licensed by Novartis seeks to manufacture the medicine. 
In the following, the data related to the model of the chapter is described. The source of the data is the data collected by the firms under consideration, for example, the annual depreciation of collaborative alliance, the reported data of Medical Equipment Department of Iran, e.g., the statistics of the population of thalassemia patients and their demand (https://www.fda.gov.ir/en), and the reported data of the Ministry of Industry, Mine and Trade of Iran (https://en.mimt.gov.ir/) to outline the data about the market share of active firms. The supports provided by the government facilitate the insurance coverage of 85 percent $(X=0.15)$ of the treatment costs. The patients pay the remaining 15 percent of the cost. Although the new medicine's market penetration (Jadenu ${ }^{\circledR}$ ) is only 5 percent, regarding its proven effectiveness and the establishment of domestic manufacturing, it seems that the new medicine will take 100 percent of the market soon. The annual depreciation rate for the foreign manufacturer is calculated as $D P_{F}=1200000 \$$. There are about 18,500 thalassemia patients in Iran who are taking medication. It should also be noted that some thalassemia patients are either in the silent phase or have mild symptoms and do not need medication. This medicine is available as 90, 180, and $360 \mathrm{mg}$ tablets. A thalassemia patient's need for the medicine varies from $7 \mathrm{mg}$ to $28 \mathrm{mg}$ per kilogram of the patient's weight per day (according to the severity of the disease and whether the patient has regular or irregular blood transfusions). Therefore, this medicine's average annual consumption is estimated to be 1,500 kg/year or 1.5 tons.

The import price of a medicine, which the government considers before the establishment of a domestic company, is calculated by comparing the price of the medicine in the reference countries $(\mathrm{Pb}=131 \$ / \mathrm{gr})$. Meanwhile, the pharmacy profit margin for this medicine is 18 percent $(p s=0.18$ ), and the gross profit margin is calculated as 35 percent for the distributor $(d s=0.35)$. The cost of production of this medicine in the country of origin is $c_{F}=43.5 \$ / \mathrm{gr}$. Taking into account the import tariff of raw materials, the cost of producing each gram of medicine by the local company is $c_{L}=48.5 \$ / g r$.

To meet the domestic need of the medicine, we need a company with the capability to produce $1,500 \mathrm{~kg}$ annually, the cost of equipment is $267,000,000 \$$. Hence, using the straight-line depreciation method and considering a 12-year lifespan for the company, the annual depreciation rate is calculated as $D P_{L}=23000000 \$$. Given the support of foreign investment in Iran, the tax 
rate of the foreign company will be equal to that of the domestic company $\left(\tau_{F}=\tau_{L}=0.25\right)$, but the tax rate in the case of no cooperation is $\tau_{F, 1}=0.3$. Also, the $R O C$ rate of the local company is estimated by considering the inflation adjustment as $R O C=0.12$.

Considering the fair distribution of the resulting synergy of the cooperation, the fair equilibrium price of the medicine in the market is calculated using Eq. (5.18) equal to $114.55 \$$, which is about $13 \%$ lower than the import price. By inserting the equilibrium price into Eq. (5.2), the license revenue is equal to $24361354.3 \$$, meanwhile, the revenue function of the foreign company after cooperation is calculated using Eq. (4) as $\pi_{F, \mathrm{C}}=17052948$ \$. On the other hand, using Eq. (5.8), the revenue function of the foreign company before cooperation is $\pi_{F, N}=4021875 \$$. Hence, it is quite evident that the coalition has made a significant profit for the foreign company. Similarly, we can compare the revenue of the local company and the government before and after the cooperation by using the developed equations in the model of the study. The results for each supply chain member before and after cooperation are presented in Table 5.1.

Table 5.1. The results of revenue distribution in equal sharing mode

\begin{tabular}{|c|c|c|c|}
\hline $\begin{array}{c}\text { Supply chain } \\
\text { member }\end{array}$ & $\begin{array}{c}\text { The profit before } \\
\text { cooperation }\end{array}$ & $\begin{array}{c}\text { The profit of } \\
\text { cooperation }\end{array}$ & $\begin{array}{c}\text { The extra profit of } \\
\text { cooperation }\end{array}$ \\
\hline F & 4021875 & 17052948 & $\mathbf{1 3 0 3 1 0 7 3}$ \\
\hline L & 25760000 & 38791073 & $\mathbf{1 3 0 3 1 0 7 3}$ \\
\hline G & -207612075 & -181549928.9 & $\mathbf{2 6 0 6 2 1 4 6 . 1}$ \\
\hline
\end{tabular}

If we set $L=0$ in Eq. 5.2, then the royalty fee will be $r=0.14$ or 14 percent. In other words, the medicine manufacturing company can grant the production license to the domestic company in exchange for $14 \%$ of its sales revenue. One common method for calculating the royalty fee is the 25 percent rule. In this method, $25 \%$ of the net profit is attributed to intellectual property. In the above case, with considering $c_{L}=48.5$ and splitting depreciation into each gram of medicine, the total cost can be calculated as $14.6+48.5=63.1 \$$. Accordingly, the license value for each gram of medicine sold is calculated as $0.25^{*}(114.55-63.1)=12.85 \$$. Also, with attributing 0.14 to the 
royalty fee, the license value is calculated as $0.14 * 114.55=16.036 \$$. Therefore, it is evident that the results of the proposed model and $25 \%$ role have a low difference. However, the proposed model provides a deep analysis of the capabilities of partners and hence has high reliability for the implementation in real cases.

(Zaharoff, 2004) reported the average royalty fee in the pharmaceutical industry as $8 \%$, which is significantly different from $14 \%$. As witnessed in the above case, the monopoly market makes both foreign and domestic producers' profits more than double compared to the pre-cooperation stage. Thus, even if domestic and foreign producers' profit rates are decreased compared to the equal sharing mode, they will probably have the motivation to cooperate.

As stated, various factors can affect the pricing process and license valuation. In the next subsections, we will analyze the problem by changing the values of the parameters.

\subsubsection{The market share of the foreign company}

According to Proposition 2, the necessary and sufficient condition for the formation of a domestic medicine manufacturing company with the permission of a foreign company is that the minimum price acceptable to the coalition $\left(p_{c}^{\min }\right)$ be lower than $p_{B}$ in Eq. (5.17). We can analyze the factors affecting this price by using Eq. (5.17). The first effective parameter is the market share of the foreign company in the domestic market before the coalition. Figure 5.2 illustrates the changes of the minimum price against the market share of the foreign company $(s)$. 


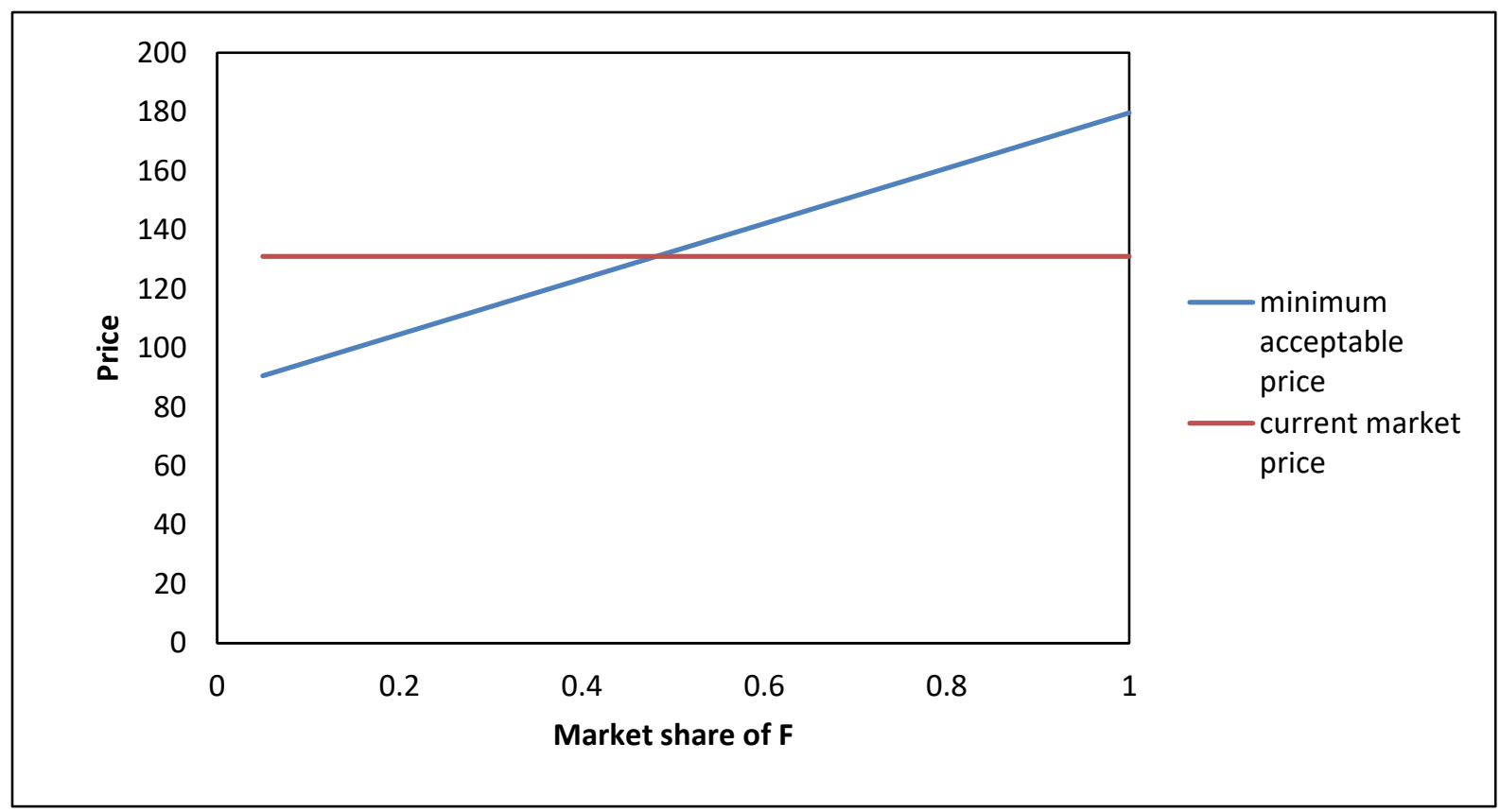

Figure 5.2. $p_{c}^{\min }$ against the market share of the foreign company

The results of Figure 5.2 show that as the market share of the foreign company increases, the minimum acceptable price for presenting a license contract to the domestic producer increases. Hence, it can be interpreted that with having a significant market share, the incentive for the foreign company to share its sales profits with the government and the domestic company would be less. Also, when the market share of the foreign company is greater than $0.4813 \%$, it gains a significant market share without coalition; but the domestic company must pay for the required investment while ensuring the profitability of the foreign company at least equal to its profit before granting the license. However, when the market share of the foreign company exceeds a certain amount (in this case $0.4813 \%$ ), the domestic company obtains no profit after paying the foreign company profits through license revenue and therefore has no motivation to purchase a medicine license for production.

The impact of the foreign company's share of the domestic market can also be examined on the equilibrium price. Figure 5.3 shows the equilibrium price of the foreign company concerning the market share. It indicates that the larger the market share of the foreign company before the coalition, the higher the equilibrium price since at a lower price than the new equilibrium price, 
the foreign company will not benefit substantially from the coalition and, in fact, will have no incentive for the partnership as the coalition. The results of Figures 5.2 and 5.3 also indicate that if the government had the right to choose from foreign companies, it would be able to provide the medicine at a lower market price by selecting a company with less market share. As a result, it can reduce patients' costs and the share of the costs that he must bear.

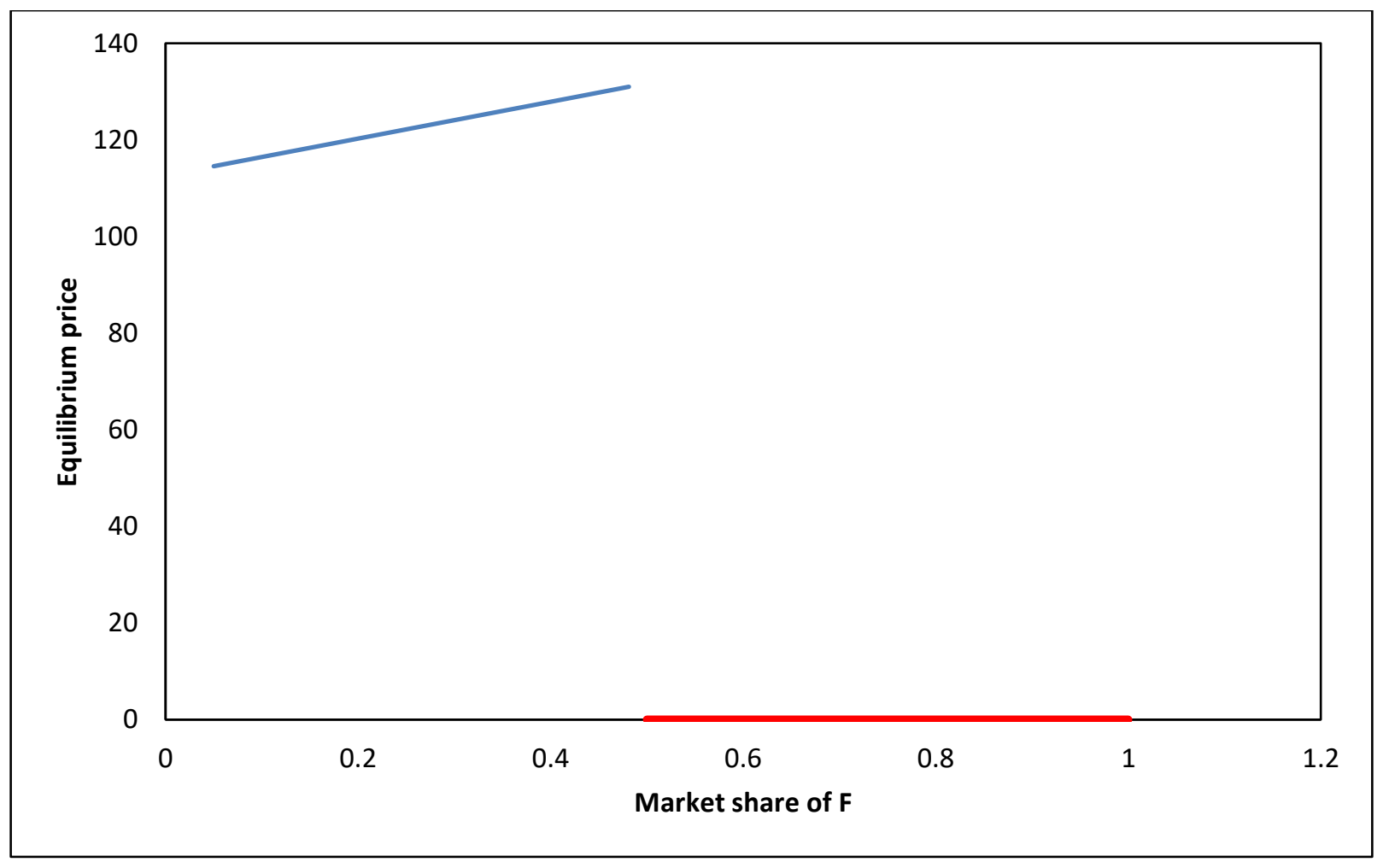

Figure 5.3. Changes in the equilibrium price relative to the market share of the foreign company

* The red line is corresponding to the market share of the foreign firm at which the cooperative strategy is not profitable.

\subsubsection{Income tax rate of the licensor}

The income tax rate of a foreign company as the licensor (which is imposed by the government on the income of a foreign company), is another factor that may affect both the formation of a domestic medicine manufacturing company and the market equilibrium price. By changing this rate, the government can stimulate the licensor's incentive for granting the license rights and effect medicine pricing. According to Eq. (5.17), with increasing the rate, the minimum acceptable price $\left(p_{c}^{\min }\right)$ will increase. Figure 5.4 displays the changes of $p_{c}^{\min }$ against changes in the foreign 
company's income tax. However, slight variations in the slope of the chart, especially in the range of the current tax rate (10-30\%), indicate that despite the government's control over this tax rate, the effect of these changes on $p_{c}^{\min }$ is insignificant.

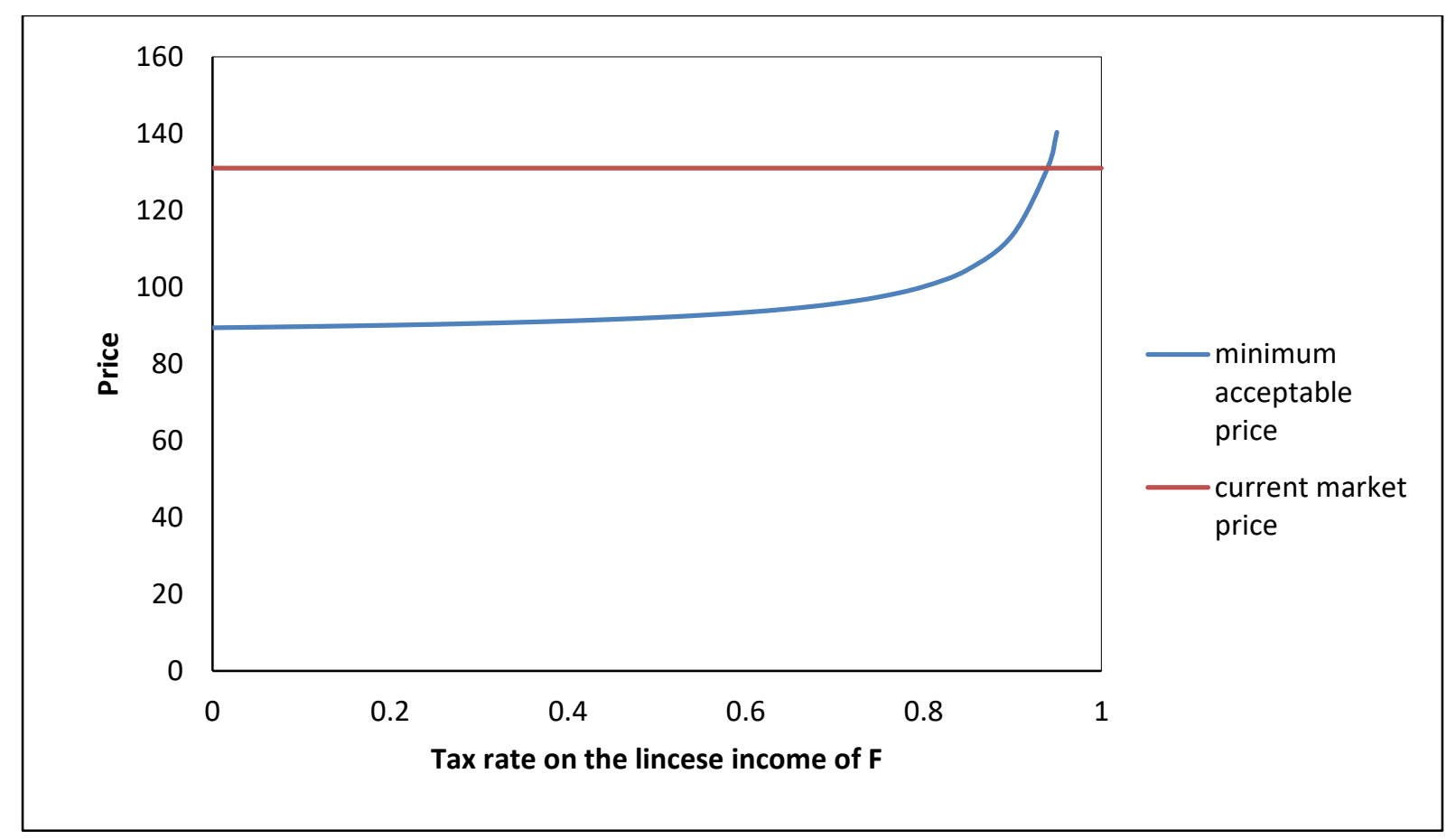

Figure 5.4 $p_{c}^{\min }$ against income tax rate of the foreign company

The effects of changing the tax rate on the equilibrium price have been shown in Figure 5.5. According to this figure, any increase in the tax rate would raise the equilibrium price. Clearly, increasing the tax rates increases the incomes of the government. However, increasing the equilibrium price as the result of increasing the tax rate has negatively increased the costs of patients afforded by the government. However, given that the tax rates of different industries, such as pharmaceutical producers, are determined in broad terms, the parameter can not be regarded as a separate variable in the model. Its effect can only be scrutinized through sensitivity analysis. Thus, it can only provide insights to the decision-makers in public economic affairs. 


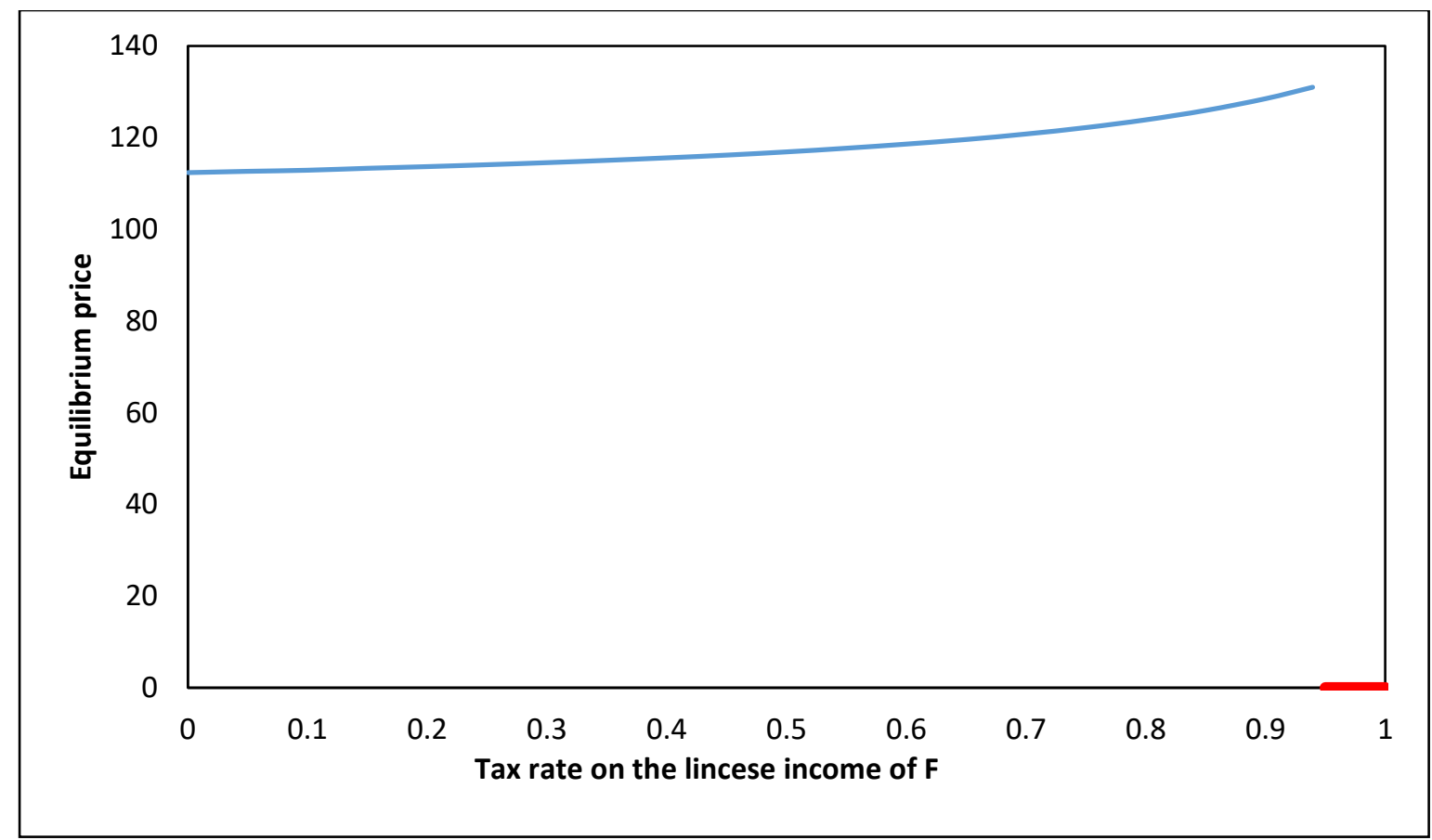

Figure 5.5. Changes in the equilibrium price in relation to income tax of the foreign company

*The red line is corresponding to the tax rate at which the cooperative strategy is not profitable.

\subsubsection{Return on capital of the local firm $\left(R O C_{\mathrm{L}}\right)$}

Another parameter that can affect the pricing and formation decisions is the rate of return on capital of the local company $\left(R O C_{\mathrm{L}}\right)$. Figure 5.6 shows the changes in the minimum acceptable price ( $p_{c}^{\min }$ ) for various values of $R O C_{\mathrm{L}}$. Subsequently, increasing the $R O C_{\mathrm{L}}$ results in higher values for $p_{c}^{\min }$. This is due to the fact that investors with higher $R O C_{L}$ request more profit for participating in the cooperative strategy to ensure a profit equal or greater than the one they could obtain in their business. In addition to the impact of $R O C_{\mathrm{L}}$ on $p_{c}^{\min }$, the effect of this parameter on the equilibrium price is verified to be significant as shown in Figure 5.7. 


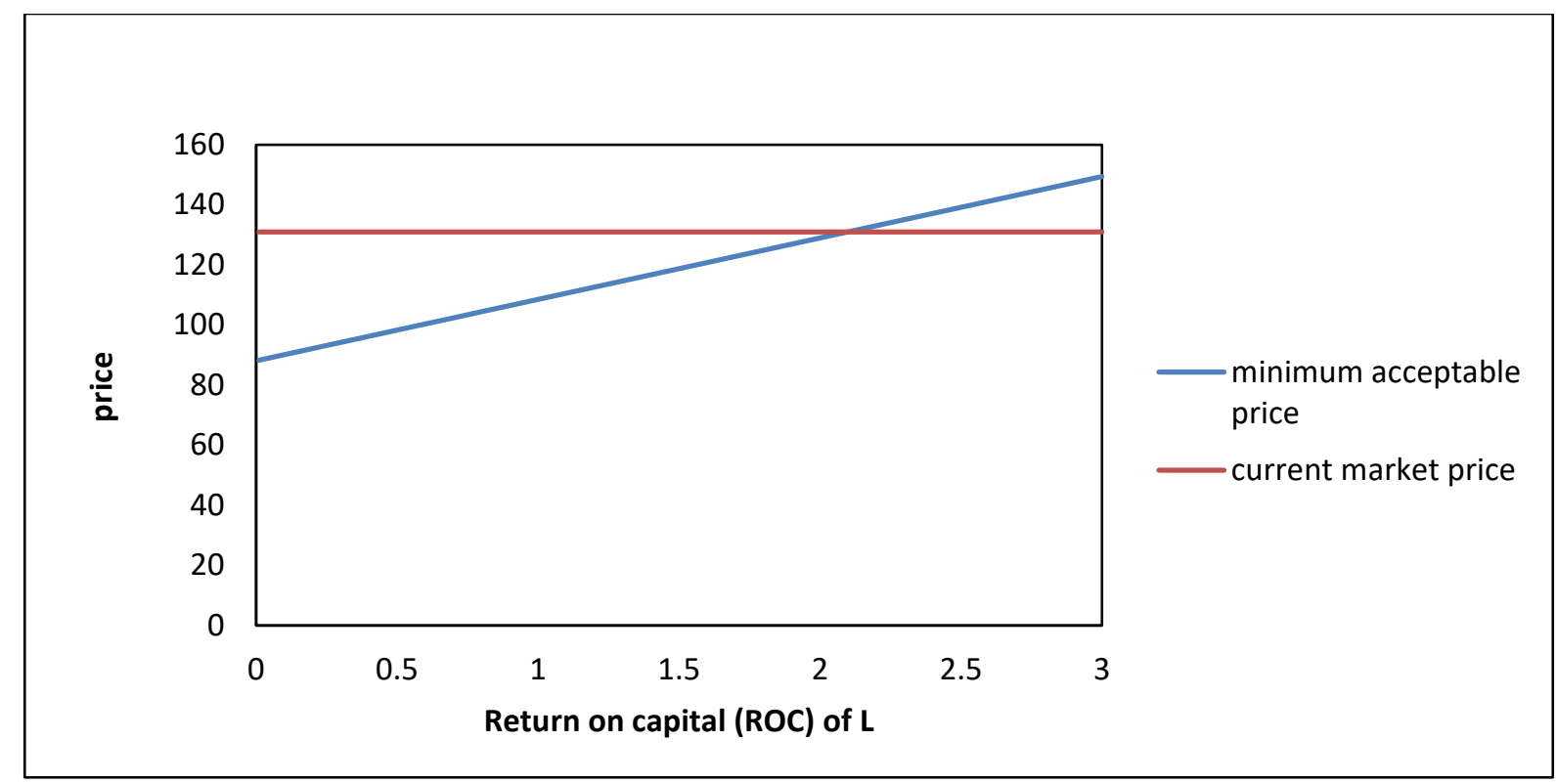

Figure 5.6. $p_{c}^{\min }$ versus $R O C_{L}$

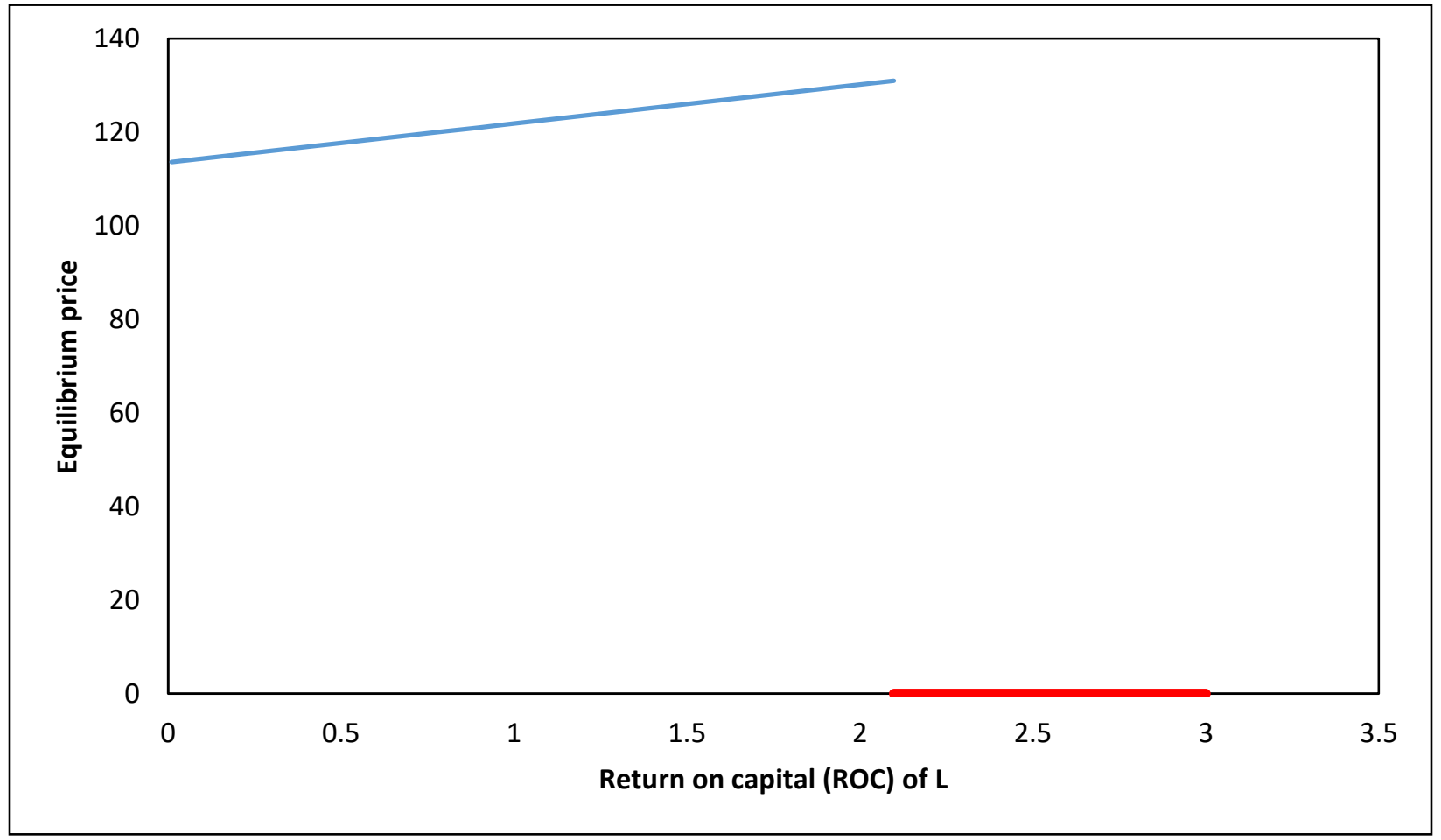

Figure 5.7. Changes in the equilibrium price against $R O C_{\mathrm{L}}$

* The red line indicates the rate of return on capital at which, the cooperative alliance is not profitable. 
The results of Figure 5.7 indicate a linear relation between $R O C_{\mathrm{L}}$ and equilibrium price. Accordingly, the smaller the $R O C_{\mathrm{L}}$, the less profit is expected for $L$ in the cooperative strategy, and this will allow the government to request a higher reduction in the price of the licensed medicine.

Evidence of the low-interest rates requested by investors and their impacts on public projects' costeffectiveness is the financing of public and private projects by state securities with the lowest rate of return. This method of financing has minimal risk for investors, while the government could reduce its costs due to the low interest rates of such state securities. Therefore, if the government can produce medicines by getting the license and providing the investment cost and license payments using government bonds, the reduction in the patients' costs will be the most. Moreover, even if licensed medicines' production requires capabilities beyond the government's ability, the domestic companies with the lowest $R O C$ rate are preferable. Knowledge-based and emerging companies can be a good option in this regard. However, a trade-off between the risk of failure by the less known companies and the cost reduction saving is needed to evaluate the partner selection issues in the cooperative strategy.

\subsection{Conclusion}

In this chapter, the cooperative strategy of producing medicine for the treatment of thalassemia was evaluated. The medicine was intended to be produced domestically by a domestic company under the main foreign manufacturer's license. For this purpose, the cooperative game theory and the Nash bargaining solution were utilized to propose a license contract considering partners' different capabilities. It was described how the pricing decisions could affect the profitability of the partners and the parameters of the license contract. Moreover, the government was introduced as another partner in the cooperative strategy, which could affect the profitability of the strategy by granting the exclusive production right to the domestically produced medicine under the license contract. So, regarding the dominant role of the government, two cooperative games were introduced: the first game between the domestic and foreign manufacturer in setting the license contract; and the second one between the cooperative alliance of manufacturers and the government in bargaining on the new price of the licensed medicine to account for the role of the government and the way it could decrease its costs by granting the required permissions to the alliance. As explained, the proposed pricing mechanism is based on real-world assumptions and 
proposes a structured framework for modification of the price such that the synergy or value resulting from the inter-firm partnership is fairly distributed. The necessary and sufficient conditions for the cooperative strategy's affordability were also derived, and the equilibrium price and the license contract parameters were determined by considering the Nash bargaining solution axioms and evaluating the two mentioned games simultaneously. Finally, we point out that the intended alliance between the manufacturers and the government could improve the throughput of the partners and increase the welfare of the patients by reducing prices and health care costs. For this reason, cooperative games for long-term relationships were utilized rather than noncooperative or competitive ones.

Besides implementing the proposed models on the case study and clarifying its result, the sensitivity analysis was carried out in two directions. The first examined the changes in the equilibrium price of the game against different values of the considered parameter. On the other hand, the second one explored the possibility of alliance formation when the parameters were subjected to deviations. Both of them also provide valuable insights about the formation of the alliance and partner selection if this is the case. For example, the results indicated that the licensor's market share on the local market and the return on the capital (ROC) of the local licensee manufacturers increase the equilibrium price of the model and decrease the possibility for the establishment of the partnership alliance. These suggest that if the rest of the conditions are the same, the best choice of government for saving its costs is the brand medicines with less market share and the local manufacturer that claims lower return on the capital. 


\section{Chapter 6: A new pricing mechanism for licensed pharmaceutical products concerning their deterioration}

The deterioration of pharmaceutical products is one of the main challenges of healthcare systems for which researchers explore solutions, including managerial and technological approaches. In this chapter, granting the production right of patented medicines is studied as a strategy that improves the logistic capabilities and increases the efficiency of the Pharmaceutical Supply Chain (PSC). The effects of this strategy are investigated in terms of the reduction in ordering costs of distributors and the exclusive production right provided by the government to a local licensed manufacturer. Considering the benefits of this strategy in the PSC, such as the reduction in the deterioration of medicines, a profit-sharing mechanism based on the contributions of members in affording the cost of the supply chain is proposed. The mechanism modifies the price of drugs charged by the manufacturer and the marginal profit of distributors and pharmacies such that members besides the government (who are the dominant legislator of the PSC), take advantage of the participation strategy appropriately. The proposed model was implemented in a real case study of a pharmaceutical pricing problem in Iran with results indicating that the strategy improved the supply chain's performance in reducing the costs and the deterioration of the drugs. Moreover, the new price and royalty rate based on the model was shown not to be far from the current setting in the PSC under consideration.

\subsection{Introduction}

One of the most challenging issues in the PSC is the deterioration of products. The shelf life of a drug is defined as the period of time that the drug's stability and functionality is over $90 \%$. The drug's expiration date also indicates when the medication loses its effectiveness and safety (Coffey, 2013). In 2003, a survey in the United States showed that about $\$ 500$ million is lost annually due to the deterioration of medications. In 2007, the Health Department of Chicago lost about \$1 million because of the deterioration of drugs (Masoumi et al., 2012). Another audit in the same year showed that pharmaceutical companies had about $\$ 2.05$ billion worth of medicines with expired dates. These pieces of evidence suggest that considering deterioration in the PSC models is of much significance. The strategies that are being developed can result in a significant decline in the deterioration rate of products, which would be beneficial for manufacturing companies as 
well as buyer organizations such as pharmacies. The present chapter and the proposed cooperative strategy grants the production right domestically through a license contract and obtaining the optimal policies of distributors and pharmacies in an integrated manner as the supply chain management practices are two innovations that addressed the mentioned challenge of the pharmaceutical supply chain effectively.

The proposed research objectives in this chapter are summarized as follows: First, granting the license of medicines to local manufacturers by forming a strategic alliance is introduced as a strategy that has positive effects on the profitability of the PSC and its members. Second, the chapter's cooperative strategy will reduce the deterioration of medicines as one of the worrying challenges of PSC. Third, the proposed pricing mechanism, which takes into account the contribution of partners in generating the synergy of PSC, is presented as an innovative pricing mechanism in the context of PSC that provides sufficient motivations and benefits for the cooperation of partners. Fourth, the license of production is regarded as an intellectual property (IP), and the valuation of this IP will be a combination of existing valuing methods with the pricing and profit distribution decisions as an iterative method.

\subsection{The proposed model}

This section's structure is organized as follows: First, some notes about the license contracts of the pharmaceutical products are mentioned, and the license contract type studied in this chapter is distinguished from other common types. Next, a general description of the model is provided. The distributor inventory managed model of distributors and pharmacies is stipulated in sub-section 6.2.3. The roles and cost functions of the government in the PSC under study are presented in subsection 6.4. Next, the license contract between the local and main manufacturers, which determines their profit in the cooperative strategy, is interpreted. Finally, the pricing and profit margin determination mechanism for identifying PSC members' profit in the cooperative strategy is illustrated.

\subsubsection{Voluntary license contract}

The most common type of license agreement in the pharmaceutical industry is the voluntary license contract. A company buys a patent from the original manufacturer to produce the patented 
drug for a specified period and within a geographical area. Meanwhile, under this contract, the licensee undertakes to pay a specified fee to the licensor. This contract protects the intellectual property rights of the inventors of essential medicines specified in the Agreement on Trade-Related Aspects of Intellectual Property Rights (TRIPS), in which any right to use the medicine is licensed by the original manufacturer. This contract provides a competitive advantage and thereby can result in significant profits for the original manufacturer; however, since it imposes higher costs on governments and patients for the supply of medicines, it may restrict the use of medicines. The inventors of essential medicines' exclusive rights are part of a universal human right called the property right. In this regard, Paragraph 2 of Article 27 of the Universal Declaration of Human Rights (UDHR) states that "everyone has the right to the protection of the moral and material interests resulting from any scientific, literary, or artistic production of which s/he is the author." Moreover, this right is under Paragraph C of Article 15 of the International Covenant on Economic, Social, and Cultural Rights (ICESCR). Due to the high costs of research and development in the pharmaceutical industry, the revenues from license agreements compensate the original manufacturer's costs and create an incentive for it to be more involved in producing new medicines. Another feature of this contract is that it is made with mutual parties' consent in exchange for financial and operational costs; also, reaching an agreement in this contract requires a bargaining process to preserve each party's rights (Forman, 2007).

Due to human rights considerations and the challenges of the World Trade Organization (WTO) members, especially in less developed countries, another type of license agreement has been proposed, which is known as a compulsory license contract. This contract, under certain conditions, allows the exploitation of the patents of some new medicines without the permission of the original manufacturer. This is in accordance with human rights considerations of public health announced in TRIPS, which take precedence over other obligations, including intellectual property rights (Baetens, 2009). Such contracts create a kind of balance between the international human rights system, particularly the right of access to pharmaceutical innovations and the protection of the intellectual property rights of the inventors of essential medicines. In fact, international human rights law lays down positive and negative obligations for governments to protect and guarantee basic human rights. Meanwhile, international intellectual property law obliges governments to respect intellectual property owners' exclusive rights and prevent infringements of third parties' exclusive rights. The TRIPS Agreement provides such flexibilities 
for member states to help them have access to essential medicines, and at the same time, fulfill international commitments under the ICESCR (Timmermans \& Hutadjulu, 2000). The commitment to supply and ensuring adequate access to essential medicines require the governments to effectively monitor pharmaceutical companies' measurements, such as the pricing procedures of emerging medicines, which could be a major barrier to access these products. In a nutshell, the main purpose of imposing all these obligations is to ensure that international agreements do not adversely affect the enjoyment of the right to health; in the event that this right (especially the right to access essential medicines) is impeded or violated due to bilateral trade agreements, it can be considered as a violation of the right to health (Forman, 2007).

Several points need to be considered after describing the two common types of licensing agreements in pharmaceutical industries. First, compulsory license contracts are made only in exceptional conditions, and they cannot be used simply because the government is unable to negotiate with the original manufacturing companies. In fact, the provisions envisaged in granting a compulsory license, such as the possibility of terminating exclusive rights and adding some exceptions, have been enacted as attempts to protect developing countries. The 20 -year exclusive right granted to the licensee under Article 33 of the TRIPS Agreement is a protocol to prevent unauthorized third parties from producing, manufacturing, using, selling, and importing the licensed products. However, this exclusive right is not an absolute right since Articles 30, and 31 impose restrictions on it. In this regard, Article 30 deals with the exceptions, and Article 31 deals with the issue of compulsory licenses. It should also be noted that the laws for the application of these exceptions and restrictions are very strict, and in practice, these regulations serve the interests of international and transnational pharmaceutical companies to guarantee people's right to access essential medicines in developing and less developed countries. Article 30 of the TRIPS Agreement states that "members may provide limited exceptions to the exclusive rights conferred by a patent, provided that such exceptions do not unreasonably conflict with a normal exploitation of the patent and do not unreasonably prejudice the legitimate interests of the patent owner, taking account of the legitimate interests of third parties" (Abbott, 2002). According to Article 31, granting compulsory licenses is the countries' responsibility, though this is not an absolute authority and must meet the conditions set out in Article 31. The following reasons are among these conditions: public health, urgent emergencies, epidemics, non-commercial public uses, compensation for anti-competitive practices, and environmental protection (World Health 
Organization, 2002). In addition, Article 31 explicitly states that before granting a compulsory license, it should be attempted to obtain the exclusive license holder's consent, except in cases of national emergency or other emergencies.

The TRIPS Agreement emphasizes public health in the Doha Declaration, which becomes useful when many developing and less developed countries are faced with critical public health issues, such as AIDS, tuberculosis, malaria, and other epidemics. Also, governments other than the less developed countries must prove the inadequacy of the production capacity for the medicine in demand for the TRIPS Council at the WTO. On the other hand, exporters of the licensed medicines by compulsory contracts must pay compensation to the original license holder (Abbott, 2002).

Based on the above explanations, the license agreement under study is among the voluntary license contract for the following reasons: a) it is not affected by the threats arising from the compulsory contracts, and b) it cannot affect the bargaining process between the parties. The first reason is that the drug under study is related to specific patients in a limited statistical population, and it is not a case of a national emergency caused by public health problems. In addition, there is the capacity to produce the drug or similar drugs in the country. The drug under study has better therapeutic effects than the existing medicines, but the existing ones produced in the country can still be a substitute for the new medicine. On the other hand, before establishing the cooperative strategy, the drug still exists in the pharmaceutical distribution network of the country as an imported product and is a well-known brand that has obtained the necessary administrative license for distribution. According to the case study, it has been decided to have a voluntary contract with both parties' full consent. The proposed model will discuss how these benefits can be shared equitably between the parties in a partnership contract. There are no anti-competitive practices in the pricing of the medicine under study either before or after the formation of the partnership. Indeed, the pricing mechanism of the medicine under study is reference-based pricing type. This type of pricing is close to the price of medicines in the reference countries after considering customs tariffs, shipping costs, and distributors' profit margins without any anti-competitive effects. Hence, another requirement related to the compulsory license contract is not needed for this medicine. 


\subsubsection{Model description}

In this study, the deterioration rate of medicines in the PSC is studied, including manufacturers (local vs. main), distributors and pharmacies, and the government. In the case study, it is attempted to evaluate the pricing mechanism and determine the marginal profit of PSC members according to a predetermined mechanism by the government.

As mentioned in the literature review, deterioration is one of the main challenges of PSC, which imposes high costs every year. In this chapter, local production through a license contract is introduced as a cooperative strategy that could have significant effects on the reduction of deterioration costs. As shown in the results, this strategy, alongside a proper managerial policy, will improve the efficiency of PSC. Moreover, the government has a determinant role in the PSC, and its decisions about controlling, auditing other members, and pricing decisions impact the performance of PSC. In the cooperative strategy introduced in this chapter, it is presumed that if the local manufacturer obtains the production license from the main manufacturer, the government will protect it by banning the other brand's imports and, instead, expects some discounts from the manufacturer to decrease its incurred costs.

It is shown that the cooperative strategy improves the efficiency of the supply chain. However, the resulting profit of cooperation should be split between partners in an acceptable way, considering the effort of partners in reducing the costs. In this chapter, two profit-sharing mechanisms are used. In valuing the license of the main manufacturer, a proportional contract based on the cooperative game theory is proposed. Second, for splitting the profit between the local manufacturer, the distributors, the pharmacies, and the government, a profit-sharing mechanism is used, which is proportional to the cost incurred by members. The second mechanism is due to the non-practicality of applying the game theory concepts in the calculation of values in different coalitions.

In the supply chain under consideration, there are three groups of players that have a critical role in preparing the patients' medicine requirements. The first group comprises distributors and pharmacies, which have the closest relationship with the patients. The costs incurred by this group include the purchasing cost, the ordering cost, the holding cost, and the deterioration cost. The cooperative strategy decreases the costs of this group, especially those related to ordering and deterioration. The manufacturers form the second group of players. In the non-cooperative 
strategy, the production is carried out by the main manufacturer. In contrast, the production under the license of the main manufacturer by the local manufacturer is followed in the cooperative strategy which yields a reduction in the production and distribution costs. The third group is the government as the main legislator in the PSC and HDS. Among the government's responsibilities in this system is the issuance of operating permissions in the system as well as the control of prices and profit margins of PSC members. In the negotiation process between the government and other PSC members, the government requests discounts in the supply price and profit margins to decrease its costs.

The benefits of cooperative strategy could be enumerated as the exclusive market for supporting the local manufacturer by the government, a decrease in the ordering transaction, and deterioration costs, and better management and control of the PSC. Hence, to describe these advantages, the processes and operations of members are described individually. In addition, the profit allocation in the cooperative strategy is presented.

The notations of the model throughout this chapter are presented as below:

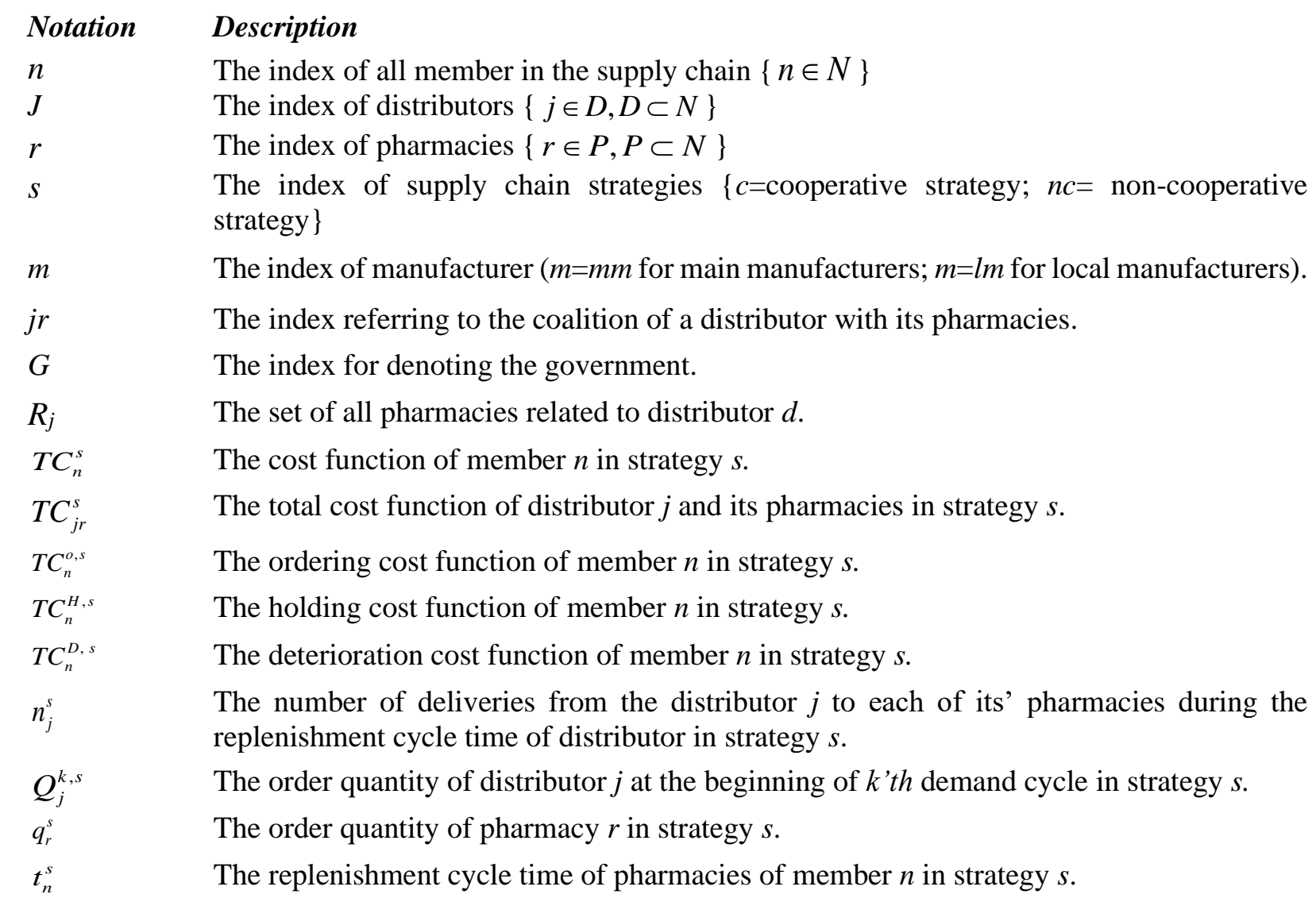




$\begin{array}{ll}I_{n}(t) & \text { The inventory level of member } n \text { at time } t . \\ D_{r} & \text { The cumulative annual demand rate of pharmacy. } \\ \theta_{n}(t) & \text { The deterioration rate of member } n . \\ C_{n}^{s} & \text { The ordering cost of member } n \text { per order in strategy } s . \\ h_{n} & \text { The holding cost of member } n \text { per unit per of time. } \\ k_{n} & \text { The deterioration cost of member } n \text { per unit time. } \\ w_{n}, \beta_{n}, \alpha_{n} & \text { The coefficients of deterioration function of member } n . \\ p_{s} & \text { The price of medicine in strategy } s . \\ c_{m} & \text { The production cost of manufacturer } m \text { per unit. } \\ D P_{m}^{s} & \text { The annual depreciation cost of manufacturer } m \text { in strategy } s . \\ R & \text { The royalty rate in the license contract. } \\ R O C_{m} & \text { The return on the capital rate of manufacturer } m \text { in its own business. } \\ Q_{s} & \text { The total order quantities of distributors in strategy } s . \\ m s & \text { The market share of the main manufacturer in the non-cooperative strategy. } \\ d s_{s} & \text { The profit margin of distributors in strategy } s . \\ p s_{s} & \text { The profit margin of pharmacies in strategy } s . \\ A_{s} & \text { The total profit margin of distributors and pharmacies in strategy } s . \\ X & \text { The percentage of patients' costs afforded by the government. } \\ P_{n} & \text { The cooperative gain of member } n . \\ W_{n}^{s} & \text { The cost function of member } n \text { in strategy } s . \\ \pi_{n}^{s} & \text { The profit function of member } n \text { in strategy } s .\end{array}$

As Child et al. (2005) stated, in valuing partnership alliances, the parties should calculate the value of their physical and spiritual assets appropriately and divide the dividends of the partnership based on the assets they provide for the alliance. Among the benefits of this alliance for the original manufacturer is that the manufacturer grants a license for the exclusive production of the medicine to the local government; on the other hand, the main advantages of domestic production include just-in-time manufacturing with less variable and fixed costs, and low distribution to provide medicines at the lowest cost possible.

It should be mentioned that although reducing the deterioration rate of medicines is not the main aim of forming a partnership alliance, it is one of the significant benefits of this partnership. In this way, one of the main challenges of the PSC, which incurs high costs, is solved. Moreover, to solve this fundamental challenge, forming a partnership alliance through a license agreement is a strategic decision that, along with the efficiency of operational decisions, can significantly improve 
the PSC and human health. Accordingly, given the reduced costs of deterioration, local governments can expect discounts from both distributors and manufacturers because of facilitating this process. According to Child et al. (2005), this is one of the competitive advantages for the government through which it can bargain with other actors.

\subsubsection{The distributors and pharmacies}

It is assumed that several distributors in the PSC that use the distributor inventory management policy. The distributors apply fixed replenishment cycle time for all their assigned pharmacies. Thus, for each distributor, one pharmacy is regarded as, such that the cumulative demands of all pharmacies of distributor are used as the demand of the representative pharmacy. In fact, the holding and ordering costs are regarded as fixed for all pharmacies of distributors. This simplification is considered because the aim of the model is to determine the policy of the government for special drugs at the macro level, and hence, considering all details of the problem makes the analysis at the macro level almost impossible.

The assumptions of the distributor-pharmacy model are as follows:

1) The annual demand rate is fixed and certain. This assumption is valid when the community of patients and their daily consumptions of specific prescription drugs are known. This assumption is supported by the real case study of Iran. Indeed, the drug under study is an effective drug for specific patients, the number of which as well as their daily consumption is known and is published in official statistics. Slight fluctuations in drug demand can also be ignored. Although the deterioration of these drugs is inevitable, it is influenced by inventory system policies such as order quantity, which in turn depends on model parameters, such as ordering cost.

2) The deterioration rate is time-dependent and changes over time. This assumption makes the model applicable to many deteriorating products, because usually, as the holding time of drugs increases, the percentage of deteriorated drugs increases.

3) The distributors are responsible for determining the policy of the distributors-pharmacies model. This policy is also common for many pharmaceutical products, because in such cases, the distributors are responsible for the deterioration of items, whereby a higher level of control is considered for them as compensation for the deterioration costs. 
4) The order quantity of the distributors is a multiple of their pharmacies' order quantity. This assumption also makes possible the extraction of the optimal policies of the distributorspharmacies model. Moreover, the periodic review inventory system of the distributors has found many applications in real-world problems (Prak et al., 2015; Taleizadeh et al., 2017).

It should also be noted that the analytical model of the distributors-pharmacies has been adopted from Wang et al. (2011) because their model introduces the time-dependent deterioration rate, which is suitable for evaluating different drugs with different deterioration rates. Hence, the objective functions and the solution approach of that model are represented without paying attention to the details (see Wang et al. (2011) for more details).

To consider the time-dependent deterioration rate for distributors and pharmacies, the deterioration functions is considered as Eqs. 6.1 and 6.2 (Wang et al., 2011) as follows. The assumption about the variability of the deterioration rate over time is consistent with research addressed the inventory policies of pharmaceutical products (Rastogi and Singh, 2019).

$$
\theta_{n}(t)=\alpha_{n}+\beta_{n} \cdot w_{n} \cdot t^{w_{n}-1} \quad n \in D \cup R
$$

According to the case study of the research, the distributors are responsible for all deterioration costs of pharmacies. This is one reason why the distributor is also responsible for managing the inventory of pharmacies. The inventory levels of a distributor and its pharmacy have been depicted in Figure 6.1 (Wang et al., 2011). 


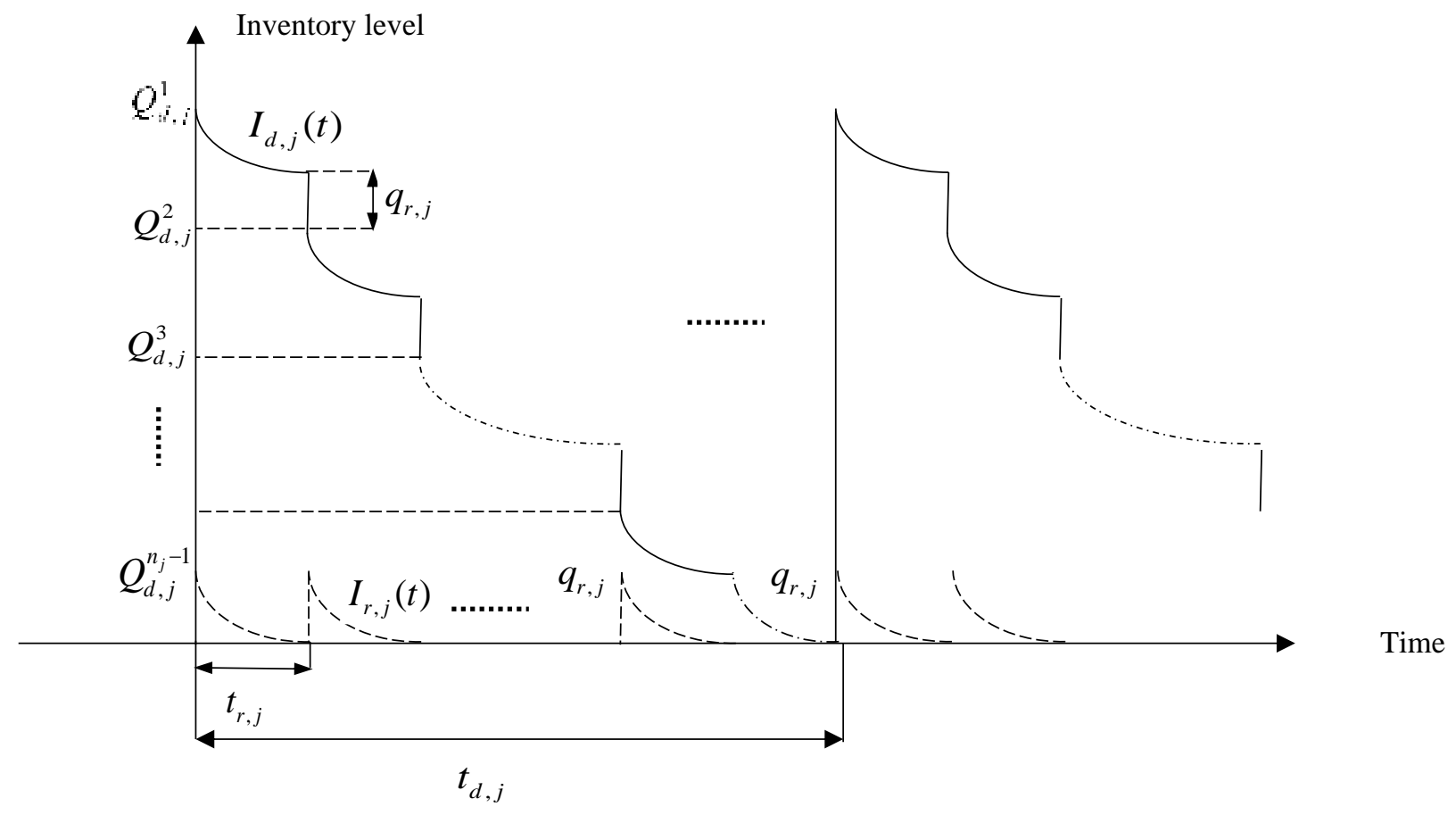

Figure 6.1 Inventory Level of distributors and pharmacies (Wang et al., 2011)

Considering Figure 6.1, it is evident that $t_{j}^{s}=n_{j}^{s} \cdot t_{r}^{s} \mid r \in R_{j}$. Furthermore, considering the above assumptions, Eqs. 2 -12 represent the total cost of distributor $j$ and its pharmacies $\left(T C_{j r}^{s}\right)$, the costs of pharmacy $r\left(T C_{r}^{s}\right)$; including the ordering cost $\left(T C_{r}^{O, s}\right)$ and holding cost $\left(T C_{r}^{H, s}\right)$; the costs of distributor $j\left(T C_{j}^{s}\right)$; including the ordering $\operatorname{cost}\left(T C_{j}^{O, s}\right)$, the total deterioration $\operatorname{costs}\left(T C_{j}^{D, s}\right)$, i.e., the sum of the deterioration cost of its pharmacies $\left(T C_{r}^{D, s} \mid r \in R_{j}\right)$ and the deterioration cost of itself $\left(T C_{j}^{D, s}\right)$; and holding cost, as well as the order quantity of pharmacies of distributor $j\left(q_{r}^{s} \mid r \in R_{j}\right)$, and the order quantity of distributor $j\left(Q_{j}^{1, s}\right)$, respectively. Interested readers could refer to Wang et al. (2011) to find more details about how different terms are embedded in the equations.

$$
\begin{aligned}
& T C_{j r}^{s}\left(C_{j}^{s}, t_{r}^{s}, n_{j}^{s}\right)=T C_{r}\left(t_{r}^{s}\right)+T C_{j}^{s}\left(C_{j}^{s}, t_{r}^{s}, n_{j}^{s}\right) \mid r \in R_{j} \\
& T C_{r}^{s}\left(t_{r}^{s}\right)=T C_{r}^{O, s}\left(t_{r}^{s}\right)+T C_{r}^{H, s}\left(t_{r}^{s}\right) \\
& T C_{r}^{O, s}\left(t_{r}^{s}\right)=\frac{C_{r}}{t_{r}^{s}}
\end{aligned}
$$




$$
\begin{aligned}
& T C_{r}^{H, s}\left(t_{r}^{s}\right)=\frac{D_{r} \cdot h_{r} \cdot t_{r}^{s}}{2} \cdot\left(1+\frac{\alpha_{r} \cdot t_{r}^{s}}{3}-\frac{\alpha_{r}^{2} \cdot\left(t_{r}^{s}\right)^{2}}{4}+\frac{2 \omega_{r} \cdot \beta_{r} \cdot\left(t_{r}^{s}\right)^{\omega_{r}}}{\left(\omega_{r}+1\right) \cdot\left(\omega_{r}+2\right)}-\frac{\alpha_{r} \cdot \beta_{r} \cdot\left(t_{r}^{s}\right)^{\omega_{r}+1}}{\left(\omega_{r}+1\right)}-\frac{\beta_{r}^{2} \cdot\left(t_{r}^{s}\right)^{2 \omega_{r}}}{\left(\omega_{r}+1\right)^{2}}\right) \\
& T C_{j}^{s}\left(C_{j}^{s}, t_{r}^{s}, n_{j}^{s}\right)=T C_{j}^{O, s}\left(C_{j}^{s}, t_{r}^{s}, n_{j}^{s}\right)+T C_{r}^{D, s}\left(t_{r}^{s}\right)+T C_{j}^{D, s}\left(t_{r}^{s}, n_{j}^{s}\right)+T C_{j}^{H, s}\left(t_{r}^{s}, n_{j}^{s}\right) \mid r \in R_{j} \\
& T C_{j}^{O, s}\left(C_{j}^{s}, t_{r}^{s}, n_{j}^{s}\right)=\frac{C_{j}^{s}}{n_{j}^{s} \cdot t_{r}^{s}} \quad \mid r \in R_{j} \\
& T C_{r}^{D, s}\left(t_{r}^{s}\right)=D_{r} \cdot k_{r} \cdot t_{r}^{s} \cdot\left(\frac{\alpha_{r}}{2}+\frac{\alpha_{r}^{2} \cdot t_{r}^{s}}{6}-\frac{\alpha_{r}^{3} \cdot\left(t_{r}^{s}\right)^{2}}{8}+\frac{\beta_{r} \cdot\left(t_{r}^{s}\right)^{\omega_{r}-1}}{\omega_{r}+1}+\frac{\alpha_{r} \cdot \beta_{r} \cdot\left(t_{r}^{s}\right)^{\omega_{r}}}{\omega_{r}+2}-\right. \\
& \left.\left.\frac{3 \alpha_{r}^{2} \cdot \beta_{r} \cdot\left(t_{r}^{s}\right)^{\omega_{r}+1}}{2\left(\omega_{r}+3\right)}+\frac{\beta_{r}^{2} \cdot\left(t_{r}^{s}\right)^{2 \omega_{r}-1}}{2\left(2 \omega_{r}+1\right)}-\frac{3 \alpha_{r} \cdot \beta_{r}^{2} \cdot\left(t_{r}^{s}\right)^{2 \omega_{r}}}{4\left(\omega_{r}+1\right)}-\frac{\beta_{r}^{3} \cdot\left(t_{r}^{s}\right)^{3 \omega_{r}-1}}{2\left(3 \omega_{r}+1\right)}\right)\right) \quad \mid r \in R_{j} \\
& T C_{j}^{D, s}\left(t_{r}^{s}, n_{j}^{s}\right)=\frac{k_{j}}{n_{j}^{s} \cdot t_{r}^{s}} \cdot\left(Q_{j}^{1}-\left(n_{j}^{s}-2\right) \cdot q_{r}^{s}-Q_{j}^{1, s} \cdot \exp \left(-\left(n_{j}^{s}-1\right) \cdot\left(\alpha_{j} \cdot t_{r}^{s}+\beta_{j} \cdot\left(t_{r}^{s}\right)^{\omega_{j}}\right)\right)\right. \\
& \left.+q_{r}^{s} \cdot \exp \left(-\alpha_{j} \cdot t_{r}^{s}-\beta_{j} \cdot\left(t_{r}^{s}\right)^{\omega_{j}}\right) \cdot \frac{1-\exp \left(-\left(n_{j}^{s}-2\right) \cdot\left(\alpha_{j} \cdot t_{r}^{s}+\beta_{j} \cdot\left(t_{r}^{s}\right)^{\omega_{j}}\right)\right)}{1-\exp \left(-\alpha_{j} \cdot t_{r}^{s}-\beta_{j} \cdot\left(t_{r}^{s}\right)^{\omega_{j}}\right)}\right) \mid r \in R_{j} \\
& T C_{j}^{H, s}\left(t_{r}^{s}, n_{j}^{s}\right)=\frac{1}{n_{j}^{s} \cdot t_{r}^{s}} \cdot \frac{h_{j} \cdot\left(Q_{j}^{1}-\left(n_{j}^{s}-2\right) \cdot q_{r}^{s}-Q_{j}^{1} \cdot \exp \left(-\left(n_{j}^{s}-1\right) \cdot\left(\alpha_{j} \cdot t_{r}^{s}+\beta_{j} \cdot\left(t_{r}^{s}\right)^{\omega_{d}}\right)\right)\right.}{\alpha_{j}+\beta_{j} \cdot w_{j} \cdot\left(n_{j}^{s} \cdot t_{r}^{s}\right)^{w_{j}-1}}+ \\
& \frac{1}{n_{j}^{s} \cdot t_{r}^{s}} \cdot \frac{q_{r}^{s} \cdot h_{j} \exp \left(-\alpha_{j} \cdot t_{r}^{s}-\beta_{j} \cdot\left(t_{r}^{s}\right)^{\omega_{j}}\right) \cdot\left(1-\exp \left(-\left(n_{j}^{s}-2\right) \cdot\left(\alpha_{j} \cdot t_{r}^{s}+\beta_{j} \cdot\left(t_{r}^{s}\right)^{\omega_{j}}\right)\right)\right)}{\left(\alpha_{j}+\beta_{j} \cdot w_{j} \cdot\left(n_{j}^{s} \cdot t_{r}^{s}\right)^{w_{j}-1}\right)\left(1-\exp \left(-\alpha_{j} \cdot t_{r}^{s}-\beta_{j} \cdot\left(t_{r}^{s}\right)^{\omega_{j}}\right)\right)} \quad \mid r \in R_{j} \\
& q_{r}^{s}\left(t_{r}^{s}\right)=D_{r} \cdot\left(t_{r}^{s}+\frac{\alpha_{r} \cdot\left(t_{r}^{s}\right)^{2}}{2}+\frac{\beta_{r, j} \cdot\left(t_{r}^{s}\right)^{\omega_{r}+1}}{\omega_{r}+1}\right) \\
& Q_{j}^{1, s}\left(t_{r}^{s}, n_{j}^{s}\right)=q_{r}^{s} \cdot \exp \left(\alpha_{j} \cdot t_{r}^{s}+\beta_{j} \cdot\left(t_{r}^{s}\right)^{\omega_{j}}\right)\left[\frac{1-\exp \left(\left(n_{j}^{s}-1\right) \cdot\left(\alpha_{j} \cdot t_{r}^{s}+\beta_{j} \cdot\left(t_{r}^{s}\right)^{\omega_{j}}\right)\right)}{1-\exp \left(\alpha_{j} \cdot t_{r}^{s}+\beta_{j} \cdot\left(t_{r}^{s}\right)^{\omega_{j}}\right)}\right]
\end{aligned}
$$

It is also notable that when a drug is manufactured exclusively in one country to meet the needs of all patients, many pharmacies (each related to one main distributor) take the responsibility of supplying the drug. It is almost impossible to check all pharmacies in detail and determine their optimal policy individually. Hence, a simple hypothesis used in model development is that the replenishment cycle time of all pharmacies belonging to a distributor is equal. Also, in the case study, the sum of the demands of pharmacies serving by a distributor is used as the demand of the representative pharmacy of that distributor. This hypothesis is intended to make the model simple and practical, but research such as Nematollahi et al. (2018) supports this hypothesis by 
considering a fixed visit interval for the distributor of a set of pharmacies. Moreover, the strategy that gives the responsibility of determining the optimal policies of two-echelon inventory systems to the vendors, known as the vendor-managed inventory system or in our case, distributor-managed inventory systems. This strategy has wide applications in the pharmaceutical supply chains and the authors such as Tsui et al. (2008), Krichanchai and MacCarthy (2017), and Hossain and Parvez (2020) have applied and assessed it.

In the above equations, the cost functions of the distributors have been introduced as a function of $C_{j}^{s}$. This because the ordering costs of distributors are different in the cooperation and noncooperation strategies, and it is expected that by reducing this parameter in the cooperation strategy, the supply chain costs will decrease remarkably. Moreover, when the value of $n_{j}^{s}$ and $t_{r}^{s}$ is determined by optimizing $T C_{j r}^{s}$ in Eq. (6.2), order quantities of the pharmacies and distributors will be specified by Eqs. (6.11) and (6.12), respectively (Wang et al., 2011).

Due to the discrete nature of $n_{j}^{s}$, the convexity of $T C_{j r}^{s}$ in Eq. (6.2) is not confirmed. However, for fixed values of $n_{j}^{s}$ the convexity of $T C_{j r}^{s}$ is proved (Wang et al., 2011). Therefore, in a modified iterative algorithm as the following, the near-optimal values of $t_{r}^{s}$, and $n_{j}^{s}$ can be determined (Wang et al., 2011):

\section{The algorithm for finding the solution of the distributor-pharmacy model:}

1) Since the number of deliveries $n_{j}^{s}$, is an integer, the heuristic starts by choosing an integer $n_{j}^{s} \geq 1$

2) Take the partial derivative of $T C_{j r}^{s}$ with respect to $t_{r}^{s}$, and equate the result to zero. That is $\partial T C_{j r}^{s}\left(C_{j}^{s}, t_{r}^{s}, n_{j}^{s}\right) / \partial t_{r}^{s}=0$

3) Substitute $t_{r}^{s}$ from Step 2 into Eq. (6.11) to derive $q_{r}^{s}$.

4) Substitute $t_{r}^{s}$ and $q_{r}^{s}$ into Eq. (6.12) to derive $Q_{j}^{1, s}$.

5) Repeat Steps 2, 3, and 4 for all possible values of $n$, until $T C_{j r}^{s}\left(C_{j}^{s}, n_{j}^{s^{*}}, t_{r}^{s^{*}}\right)$; the minimum value of $T C_{j r}^{s}$ by the algorithm; is found. $T C_{j r}^{s}\left(C_{j}^{s}, n_{j}^{s^{*}}, t_{r}^{s^{*}}\right)$ satisfies the conditions 
$T C_{j r}^{s}\left(C_{j}^{s}, n_{j}^{s^{*}}, t_{r}^{s^{*}}\left(n_{j}^{s^{*}}\right)\right) \leq T C_{j r}^{s}\left(C_{j}^{s}, n_{j}^{*}-1, t_{r, j}^{*}\left(n_{j}^{*}-1\right)\right)$

and

$$
T C_{j r}^{*_{s}}\left(C_{j}^{s}, n_{j}^{s^{*}}, t_{r}^{s^{*}}\left(n_{j}^{s^{*}}\right),\right) \leq T C_{j r}^{*_{s}}\left(C_{j}^{s}, n_{j}^{s^{*}}+1, t_{r}^{s^{*}}\left(n_{j}^{*_{s}}+1\right)\right) .
$$

6) Derive $T C_{j r}^{s}\left(C_{j}^{s}, t_{r}^{s^{*}}, n_{j}^{s^{*}}\right), T C_{r}^{s}\left(t_{r}^{s^{*}}\right)$, and $T C_{j}^{s}\left(C_{j}^{s}, t_{r}^{s^{*}}, n_{j}^{s^{*}}\right)$ using Eqs.(6.2), (6.3), and (6.6) respectively, and report them.

In the following, $t_{r}^{s}, n_{j}^{s}$ and $q_{r}^{s}$ are referred to the optimal values of the variables extracted by the above algorithm. Also, $T C_{j r}^{s}, T C_{r}^{s}$, and $T C_{j}^{s}$ will represent the optimal values of these cost functions by the proposed algorithm. In other words, the proposed model of this section acts as a complementary procedure that could extract the optimal policies of the distributors-pharmacies inventory system in both cooperative and non-cooperative strategies. However, this is not influenced by the profit split mechanisms discussed later but they do affect the split decisions.

\subsubsection{The government}

As mentioned above, the government uses the reference-based pricing mechanism as one of the main common mechanisms in recent years. If the profit margins of distributors and pharmacies are denoted by $d s_{s}$ and $p s_{s}$, respectively, and the price of manufacturers is $p_{s}$, the price of the drug in the pharmacies for the patients will be $\left(1+p s_{s}\right) \cdot\left(1+d s_{s}\right) \cdot p_{s}$. The parameter $A_{s}$ is also introduced as $A_{s}=\left(1+p s_{s}\right) \cdot\left(1+d s_{s}\right)$ to indicate the total profit margin of the distributors-pharmacies set. Now, government costs could be clarified. The annual demand for the considered drug is $\sum_{j} \sum_{r \in J} D_{r}$.

Moreover, it is assumed that the percentage of patients' costs paid by the government is denoted by $100 x \%$. Hence, the government cost is shown in Eq. (6.13):

$$
\left.W_{G}\left(A_{s}, p_{s}\right)=x \cdot\left(1+p s_{s}\right) \cdot\left(1+d s_{s}\right)\right) \cdot p_{s} \cdot \sum_{j} \sum_{r \in J} D_{r}=x \cdot A_{s} \cdot p_{s} \cdot \sum_{j} \sum_{r \in J} D_{r}
$$

According to Eq. (6.13), if the value of $A_{s .} p_{s}$ is reduced, the cooperative strategy reduces the incurred cost of the government. 


\subsubsection{The manufacturers}

Given that in the production of medicine, technical knowledge is an essential component of production, a valuation process is needed to determine the value of the technical knowledge of the main manufacturer. In this chapter, a license contract based on the royalty rate is investigated for issuing the technical knowledge of production. The contract saves a percentage of total sales as the royalty rate $(r)$ for the main manufacturer and is the most common form of the license agreement in the pharmaceutical industry.

To identify $r$, the proportional contract of Sercu (2008) is utilized. The parameters of this contract are determined such that the excess profit of cooperation, compared to the non-cooperation style, splits between two partners equally. In other words, if the profits of the main and local manufacturers after cooperation are denoted by $\pi_{m m}^{c}$ and $\pi_{l m}^{c}$, respectively, and those of noncooperation are stated by $\pi_{m m}^{n c}$ and $\pi_{l m}^{n c}$, then the profit of cooperation is split between the main and the local manufacturer based on Eq. (6.14):

$$
\pi_{m m}^{c}-\pi_{l m}^{c}=\pi_{m m}^{n c}-\pi_{l m}^{n c}
$$

To determine $\pi_{l m}^{c}$, it is assumed that based on the agreements between the main and the local manufacturer as well as the local government, the market structure after issuing the license contract will be exclusive, and not under control by the government. Now, denoting the order quantity of distributer $j$ and its pharmacies in the case of cooperation by $Q_{j}^{1, c}$ and $q_{r}^{c} \mid r \in R_{j}$, the cumulative order quantities of the distributor $Q_{c}$ will be equal to $Q_{c}=\sum_{j}\left(Q_{j}^{1, c}+\sum_{r \in R_{j}} q_{r}^{c}\right) / t_{j}^{c}$. Also, regarding the notations of this chapter, the profits of main and local manufacturers in the cooperative strategy will be as Eqs. (6.15) and (6.16):

$$
\begin{aligned}
& \pi_{m m}^{c}=r \cdot p_{c} \cdot Q_{c} \\
& \pi_{l m}^{c}=\left(p_{c}-c_{l m}\right) \cdot Q_{c}-D P_{l m}^{c}-r \cdot p_{c} \cdot Q_{c}
\end{aligned}
$$

Eq. (6.15) expresses the license income of the main manufacturer while Eq. (6.16) represents the local manufacturer's profit which is the sales income minus the production cost, depreciation cost, and the license cost paid to the main manufacturer. 
On the other hand, in the non-cooperative strategy, the local manufacturer has no share in the market of the considered drug. Therefore, its profit should only be calculated based on the profitability of that firm on its own business. Therefore, the presumed profit of the local manufacturer for investing $D P_{l m}$ in its own business (instead of the established firm in the cooperative strategy) considering its return on the capital rate (ROC) is as Eq. (6.17). This rate for a firm is the after-tax net profit and divided by its assets. The $R O C$ is an appropriate rate for calculating the profit of the local manufacturer outside the cooperative alliance. This is due to the fact that this rate could be extracted from the accounting statements of firms and could be used to compare the performance of firms with the outside environment instead of internal goals. This assumption is also supported by Januj et al. (2019) in explaining how the firms could transform their income to capitals.

$$
\pi_{l m}^{n c}=R O C_{l m} . D P_{l m}^{c}
$$

Considering that the main manufacturer only issues its technical knowledge and does not invest in the new manufacturing firm, its profit before the cooperation will be equal to zero if it has no market share in the local country. However, if its share in the local market before forming the alliance is denoted by $s$, its profit before the cooperation is the net sales income determined according to Eqs. (6.18) and (6.19):

$$
\begin{aligned}
\pi_{m m}^{n c} & = \begin{cases}\left(\left(p_{n c}-c_{m m}\right) \cdot m s \cdot Q_{n c}-D P_{m m}^{n c}\right) & s>0 \\
0 & s=0\end{cases} \\
Q_{n c} & =\sum_{j} \frac{\left(Q_{j}^{1, n c}+\sum_{r \in R_{j}} q_{r}^{n c}\right)}{t_{j}^{n c}}
\end{aligned}
$$

Using Eqs. (6.15) - (6.19) and substituting $\pi_{m m}^{c}, \pi_{l m}^{c}, \pi_{m m}^{n c}, \pi_{l m}^{n c}$ into Eq. (6.14), the royalty rate based on the proportional contract is obtained as Eq. (6.20):

$$
r= \begin{cases}\frac{\left(\left(p_{c}-c_{l m}\right) \cdot Q_{c}-D P_{l m}^{c}\right)+\left(\left(p_{n c}-c_{m m}\right) \cdot m s \cdot Q_{n c}-D P_{m m}^{n c}\right)-D P_{l m}^{c} \cdot R O C_{l m}}{2 p_{c} \cdot Q_{c}} & s>0 \\ \frac{\left(\left(p_{c}-c_{l m}\right) \cdot Q_{c}-D P_{l m}^{c}\right)-D P_{l m}^{c} \cdot R O C_{l m}}{2 p_{c} \cdot Q_{c}} & s=0\end{cases}
$$


Substituting Eq. (6.20) into Eq. (6.16), the local manufacturer's profit in the cooperative strategy is a function of $p_{c}$ and is shown in Eq. 6.21. This price, as explained later, will be determined based on the profit allocation mechanism.

$$
\begin{aligned}
& \pi_{l m}^{c}\left(p_{c}\right) \\
& = \begin{cases}\frac{1}{2}\left(\left(p_{c}-c_{l m}\right) \cdot Q_{c}-D P_{l m}\right)-\frac{1}{2}\left(\left(p_{n c}-c_{m m}\right) \cdot m s \cdot Q_{n c}-D P_{m m}\right)+\frac{1}{2}\left(D P_{l m} \cdot R O C_{l m}\right) & s>0 \\
\frac{1}{2}\left(\left(p_{c}-c_{l m}\right) \cdot Q_{c}-D P_{l m}\right)+\frac{1}{2}\left(D P_{l m} \cdot R O C_{l m}\right) & s=0\end{cases}
\end{aligned}
$$

Also, taking into account $r$ in Eq. (6.20), the incurred cost of the local manufacturer in the case of cooperative strategy $W_{l m}^{c}$ is given in Eq. (6.22). As explained earlier, this cost consists of the production cost, depreciation cost, and the license costs based on the proportional contract.

$$
W_{l m}^{c}= \begin{cases}\frac{1}{2}\left(\left(p_{c}+c_{l m}\right) \cdot Q_{c}+D P_{l m}^{c}\right)+\frac{1}{2}\left(\left(p_{n c}-c_{m m}\right) \cdot m s \cdot Q_{n c}-D P_{m m}^{n c}\right)-\frac{1}{2}\left(D P_{l m}^{n c} \cdot R O C_{l m}\right) & s>0 \\ \frac{1}{2}\left(\left(p_{c}+c_{l m}\right) \cdot Q_{c}+D P_{l m}^{c}\right)-\frac{1}{2}\left(D P_{l m}^{c} \cdot R O C_{l m}\right) & s=0\end{cases}
$$

\subsubsection{Profit split}

The important problem in the current study is the distribution of the profit between the partners in the case of implementing the cooperative strategy. If the price and profit margins of distributors and pharmacies remain unchanged when the exclusive production is granted, the manufacturers, distributors, and pharmacies benefit from it, but the government obtains nothing. Therefore, to benefit all members appropriately, the price, as well as the profit margins, should be modified. As mentioned earlier, two profit-sharing mechanisms are utilized in this chapter, namely, the cooperative game theory and the distribution based on the afforded cost of partners. The first mechanism is used to determine the value of the technical knowledge of the main manufacturer, and the second mechanism is applied to modify the price and the profit margins. The second mechanism is used because, in contrast to the first mechanism, it is not possible to determine the values of different coalitions between members; however, it is possible to do so in the first mechanism. 
Regarding the profit split mechanisms, some points should be mentioned. For a less complicated problem, the game theory approaches such as sequential bargaining models (Yan, 2012; Yan and Yang, 2012), cooperative game theory based on the Shapley value (Sun et al., 2019, Zhang et al., 2019), core concept (Wu et al., 2017; Marzband et al., 2017), and competitive game theory (Müller and Zaby, 2019) could be referred. Moreover, for more complex problems in which the value of coalitions by different players or the value of different strategies of players could not be determined straightforwardly, other logical PAP mechanisms have been applied. For example, Moon et al. (2015) proposed that the generated profit of cooperation in supply chains could be split between the members proportional to their profits before the cooperation with the entire profit of the noncooperated supply chain. Hammani and Frein (2015) assigned the resulted profit of an integrated supply chain to members based on their contribution to affording the supply chain cost. In the model proposed in the present paper, valuing the branded drugs in the negotiations process between the local manufacturer and the main manufacturer is based on the proportional contract of Sercu et al. (2008), which is according to the cooperative game theory. However, since the profit of the government in the PSC could not be easily measured and the value of coalitions for different sets of players could not be estimated (for example, when the government takes the responsibility of supplying drugs without the participation of the distributor), the profit distribution between the government, distributors, and pharmacies as well as the licensee local manufacturers is carried out proportional to their afforded costs of the PSC. The reason is that the government only pays some portion of the patients' cost as its social responsibility."

On the other hand, the mechanism for distributing profits based on their activity, which the costs incurred by members measure, is proposed in the paper, and some valid examples are provided in the literature (Moon et al., 2015; Hammani and Frein 2015). This mechanism seems fairer, unlike Nash bargaining, which distributes the revenue of the partnership equally. In fact, this mechanism was proposed by one of the experts (author of numerous articles), who was one of the executive managers of the case study. However, if the explanation provided is not convincing, in the next edition, we can use the Nash mechanism, which requires a radical change in the model and results, and of course, is more time-consuming. 
Regarding the price and profit margins after and before the cooperation and setting $W_{G}^{c}=W_{G}\left(A_{c}, p_{c}\right)$ and $W_{G}^{n c}=W_{G}\left(A_{n c}, p_{n c}\right)$, the government's profit after applying the profit-sharing mechanism is based on Eq. (6.23):

$$
P_{G}=W_{G}^{n c}-W_{G}^{c}
$$

One of the cooperation advantages was mentioned as the reduction in the ordering cost of distributors. Accordingly, this alters the optimal policy of the distributors-pharmacies model and decreases $T C_{j}$. Therefore, to account for the profit of the distributors-pharmacies model, the Eqs. (6.24) - (6.25) are proposed.

$$
\begin{aligned}
& \pi_{j r}^{s}\left(A_{s}, p_{s}, C_{j}^{s}, t_{r}^{s}, n_{j}^{s}, Q_{s}\right)=\sum_{j} A_{s} \cdot p_{s} \cdot D_{j}-\sum_{j} p_{s} \cdot Q_{s}-\sum_{j} T C_{j}^{s}\left(C_{j}^{s}, t_{r}^{s}, n_{j}^{s}\right) \\
& W_{j r}^{s}\left(p_{s}, C_{j}^{s}, t_{r}^{s}, n_{j}^{s}, Q_{s}\right)=\sum_{j} p_{s} \cdot Q_{s}+\sum_{j} T C_{j}^{s}\left(C_{j}^{s}, t_{r}^{s}, n_{j}^{s}\right)
\end{aligned}
$$

Considering Eqs. (6.24) and (6.25), the cooperative gain of distributors and pharmacies is specified by Eq. (6.26):

$$
P_{j r}=\pi_{j r}^{c}-\pi_{j r}^{n c}
$$

Also, regarding the explanations in section 6.2.3, the additional profit of local manufacturers in the cooperative strategy is denoted by Eq. (6.27). Its cost in Eq. (6.22) is also a valid equation for the profit allocation problem.

$$
P_{l m}=\pi_{l m}^{c}-R O C_{l m} \cdot D P_{l m}^{c}
$$

The profit split between the government, the distributors, the pharmacies, and the local licensee manufacturer will be according to their afforded costs. Hence, the profit distributes between members, and the parameters of $p_{c}$ and $A_{c}$ are determined such that Eq. (6.28) is satisfied:

$$
\frac{P_{G}}{W_{G}^{c}}=\frac{P_{l m}}{W_{l m}^{c}}=\frac{P_{j r}}{W_{j r}^{c}}
$$

To derive the necessary and sufficient conditions for the cost-effectiveness of the considered strategy, two conditions should be considered. Also, in order to explain these conditions, two prices, namely $p_{1}$ and $p_{2}$ are introduced, $p_{1}$ is the price that makes the profit of the local manufacture before and after the cooperative strategy equals zero, i.e., for $p_{1}$, the relation $\pi_{l m}^{c}\left(p_{1}\right)-\pi_{l m}^{n c}=0$ is true. On the other hand, $p_{2}$ is defined as the price that establishes the relation 
$\pi_{d r}^{c}\left(A_{c}, p_{2}, C_{j}^{c}, t_{r}^{c}, n_{j}^{c}, Q_{j}^{1, c}\right)-\pi_{d r}^{n c}\left(A_{n c}, p_{n c}, C_{j}^{n c}, t_{j}^{n c}, n_{j}^{n c}, Q_{j}^{1, n c}\right)=0$. Now, if $p_{n c} \geq p_{1}$ in the first condition, then the strategy is more cost-effective in the current setting, and the profit allocation mechanism preserves the previous profit of the members and just assigns some additional profit to the members based on a logical proportion. However, if $p_{n c}<p_{1}$ but $p_{2}>p_{1}$, as the second condition, then by setting the new price as $p$ such that $p_{1} \leq p \leq p_{2}$, the profitability of the supply chain in the cooperative strategy based on the proposed profit distribution mechanism is still ensured. The advantage of the strategy will be in terms of reducing distribution costs. Out of these conditions, the strategy is not cost-effective. So, Proposition 6.1 is stated as below:

Proposition 6.1: The necessary and sufficient condition for the cost-effectiveness of the studied strategy is that either $p_{n c} \geq p_{1}$ or $p_{n c}<p_{1}$ and $p_{2}>p_{1}$.

The new price $p_{\mathrm{c}}$ and profit margin $A_{c}$ is set by solving Eq. (6.28). On the other hand, due to the fact that the government is the legislator of the supply chain, it could either apply all the changes in the profit margin for the distributors or set new $d s_{c}$ and $p s_{c}$ using the profit allocation mechanism based on the costs of distributors and pharmacies. To do so, the profit and cost functions of pharmacies and distributors are introduced as Eqs. (6.29) - (6.32), respectively:

$$
\begin{aligned}
& \pi_{r}^{s}\left(p_{s}, A_{s}, d s_{s}, p s_{s}, t_{r}^{s}\right)=\sum_{j} \sum_{r \in R_{j}} D_{r} \cdot\left(1+d s_{s}\right) \cdot\left(1+p s_{s}\right) \cdot p_{s}-\sum_{j} \sum_{r \in R_{j}} \frac{q_{r}^{s}}{t_{r}^{s}} \cdot\left(1+d s_{s}\right) \cdot p_{s}-\sum_{j} T C_{r}^{s}\left(t_{r}^{s}\right) \\
& W_{r}^{s}\left(p_{s}, d s_{s}, t_{r}^{s}\right)=\sum_{j} \sum_{r \in R_{j}} \frac{q_{r}^{s}}{t_{r}^{s}} \cdot\left(1+d s_{s}\right) \cdot p_{s}+\sum_{j} T C_{r}^{s}\left(t_{r}^{s}\right) \\
& \pi_{j}^{s}\left(p_{s}, A_{s}, C_{j}^{s}, d s_{s}, n_{j}^{s}, t_{r}^{s}, Q_{s}\right)=\sum_{j} \sum_{r \in R_{j}} \frac{q_{r}^{s}}{t_{r}^{s}} \cdot\left(1+d s_{s}\right) \cdot p_{s}-\sum_{j} T C_{j}^{s}\left(C_{j}^{s}, t_{r}^{s}, n_{j}^{s}\right)-p_{s} \cdot Q_{s} \\
& W_{j}^{s}\left(p_{s}, C_{j}^{s}, n_{j}^{s}, t_{r}^{s}, Q_{s}\right)=\sum_{j} T C_{j}^{s}\left(C_{j}^{s}, t_{r}^{s}, n_{j}^{s}\right)+p_{s} \cdot Q_{s}
\end{aligned}
$$

Then, the cooperative gain of distributors and pharmacies is obtained using Eqs. (6.33) and (6.34):

$$
\begin{aligned}
& P_{r}=\pi_{r}^{c}-\pi_{r}^{n c} \\
& P_{j}=\pi_{j}^{c}-\pi_{j}^{n c}
\end{aligned}
$$

Now, based on the profit split mechanism between distributors and pharmacies, the new profit margins $d s_{c}$ and $p s_{c}$ are derived using Eqs. (35) and (36):

$$
\frac{P_{r}}{W_{r}^{c}}=\frac{P_{j}}{W_{j}^{c}}
$$




$$
A_{c}=\left(1+p s_{c}\right) \cdot\left(1+d s_{c}\right)
$$

After introducing the models and the profit-sharing mechanism, the numerical results and model validation are investigated in the next section by conducting a case study.

\subsection{Numerical results}

The case study of this chapter is related to the production of a licensed medicine for the treatment of thalassemia patients in Iran. Thalassemia is a malignant disease with symptoms such as a low level of alpha and beta proteins in red blood cells. Hemoglobin, as the main component of red blood cells, is responsible for carrying oxygen throughout the body and producing alpha and beta proteins. The functionality of hemoglobin is disrupted in thalassemia patients, and the proteins are not sufficient in the blood cells of patients. Thalassemia patients are classified based on the deficiency and severity of the proteins in their blood. Unfortunately, thalassemia is not fully curable, and the current drugs only decline the progression and worsening of the disease. The bestknown treatment for thalassemia is blood transfusion, which has undesirable consequences and side effects such as transfusion-induced iron overload in the patient's body; the reason is that there is no way for removing iron from the body. Jadenu ${ }^{\circledR}$ (deferasirox) is one of the newly developed drugs for this disease produced under the license of Novartis. The better performance of Jadenu® than other available drugs has been confirmed by strict and rigorous quality control and stability tests, for example, the Food and Drug Administration (FDA) standards. Therefore, Iran's FDA has granted exclusive production rights to the local manufacturer under the license of Novartis.

For thalassemia, the percentage of costs incurred by the government in Iran is $85 \%(x=0.85)$. Before the domestic production of Jadenu ${ }^{\circledR}$, the market share of the drug in Iran was about $5 \%$ $(s=0.05)$. However, the whole market shares of the brand, as well as the import prevention of other brands, were ensured by the government, providing the under-license domestic production of the drug. The current profit margin of distributors for the imported drugs of Jadenu ${ }$ is $7 \%\left(d s_{n c}=\right.$ $0.07)$ while that of the pharmacies is $18 \%\left(p s_{n c}=0.18\right)$. The drug is supplied in 90,180 , and 360 $\mathrm{mg}$ tablets, and the drug requirement of the patients varies from $7 \mathrm{mg}$ to $28 \mathrm{mg}$ per kilogram of the patient's weight. There are four main distributors for the drugs, and the total demand for their 
pharmacies is estimated to be $1500 \mathrm{~kg}$ per year. The values of parameters for the considered case study are summarized in Table 6.1 .

Table 6.1 The value of parameters in the case study

\begin{tabular}{|c|c|}
\hline Parameters & Value \\
\hline$D$ & $\{1,2,3,4\}$ \\
\hline$P$ & $\{1,2,3,4\}$ \\
\hline$\left[R_{1}, R_{2}, R_{3}, R_{4}\right]$ & {$[\{1\},\{2\},\{3\},\{4\}]$} \\
\hline$D P_{m m}^{n c}$ & $\$ 36,650$ \\
\hline$D P_{l m}^{c}$ & $\$ 702,300$ \\
\hline$m s$ & 0.05 \\
\hline$p_{n c}$ & $4 \$ / g r$ \\
\hline$p s_{n c}$ & 0.18 \\
\hline$d s_{n c}$ & 0.07 \\
\hline$A_{n c}$ & 1.2626 \\
\hline$\left[D_{1}, D_{2}, D_{3}, D_{4}\right]$ & {$[240,315,810,135] \mathrm{kg}$} \\
\hline$c_{l m}$ & $\$ /$ gr 1.15 \\
\hline$c_{m m}$ & \$/gr 1.55 \\
\hline$\left[\alpha_{r}, \beta_{r}, w_{r}\right]$ & {$[0.04,0.08,1.5]$} \\
\hline$\left[\alpha_{j}, \beta_{j}, w_{j}\right]$ & {$[0.02,0.05,1.2]$} \\
\hline$\left[k_{j}, k_{r}\right]$ & $\$ /$ gr $[3.2,3.8]$ \\
\hline$\left[h_{j}, h_{j}\right]$ & \$/gr/year $[0.45,0.6]$ \\
\hline$\left[C_{j}^{c}, C_{j}^{n c}\right]$ & $\$ /$ order $[12000,500]$ \\
\hline$C_{r}$ & $\$$ /order 500 \\
\hline$R O C_{l m}$ & 0.12 \\
\hline
\end{tabular}

As shown in Table 6.1, the production cost is specified by the transaction cost of manufacturers per each supplied drug. Therefore, the lower cost of domestic production is due to the lower cost of transactions. Also, the cost of depreciation in the domestic production is much higher, because the production capacity must meet the total market demand. Another note about data is that the deterioration rate parameters indicate that as the shelf life of drugs increases, so does their rate of deterioration.

First, the optimal policy of the distributors and pharmacies in the non-cooperative strategy is investigated. Using the introduced iterative algorithm in section 6.2.3, the optimal policies of each 
distributor and pharmacy are determined (Table 6.2). As depicted in Table 6.2, the deterioration quantity in PSC is $1513630-1500000=13630 \mathrm{gr}$, which is a significant percentage of the total supplied quantity. On the other hand, regarding the value of parameters in the cooperative strategy, the inventory policy of the distributor-pharmacy model is expressed according to Table 6.3.

In the cooperative strategy, the deterioration quantity is $1503254-150000=3254 \mathrm{gr}$. The results of Table 6.4 clearly show that the cooperative strategy decreases the deterioration quantity as well as the inventory costs. Based on the cooperative strategy, the deterioration has been reduced by more than 76 percent. This result is striking and valuable in the context of PSC. For example, as expressed in the literature review, the preservation technology could decrease the deterioration by just up to $20 \%$. Based on this result, one of the main goals in supporting domestic production under license agreements can be considered a significant reduction in the deterioration of drugs. Accordingly, health system managers should take the necessary protections to facilitate the implementation of this strategy and can use the models presented in this chapter to measure and evaluate the outcomes of the strategy. Other variables of the model have been summarized in Table 6.4.

Based on the results that $p_{n c}>p_{1}$, and therefore, based on Proposition 6.1, the necessary and sufficient condition for the cost-effectiveness of the cooperative strategy exists. Also, the royalty rate of 0.2 is a common choice in licensing pharmaceutical products (Zaharoff, 2004) and is very close to the thumbnail rule of a $25 \%$ royalty rate.

Table 6.2 The optimal policy of distributors and pharmacies in the non-cooperative strategy

\begin{tabular}{|c|c|c|c|c|c|}
\hline$j$ & $\mathbf{1}$ & $\mathbf{2}$ & $\mathbf{3}$ & $\mathbf{4}$ & Sum \\
\hline$n_{j}^{n c^{*}}$ & 6 & 6 & 6 & 6 & \\
\hline$t_{r}^{n c^{*}}$ & 0.078 & 0.069 & 0.043 & 0.104 \\
\hline$t_{j}^{n c^{*}}$ & 0.473 & 0.415 & 0.261 & 0.626 \\
\hline$q_{r}^{n *^{*}}$ & 18981 & 21875 & 35282 & 14139 & \\
\hline$Q_{j}^{1, n c^{*}}$ & 96038 & 110503 & 177494 & 71856 & \\
\hline$q_{r}^{n c^{*}} / t_{r}^{n c^{*}}$ & 240548 & 315620 & 810940 & 135427 & 1502535 \\
\hline$\left(Q_{j}^{1, n c^{*}}+q_{r}^{n c^{*}}\right) / t_{j}^{n c^{*}}$ & 242943 & 318334 & 815075 & 137278 & 1513630 \\
\hline$T C_{r}^{n c}$ & $\$ 12027$ & $\$ 13773$ & $\$ 22073$ & $\$ 9028$ & $\$ 56901$ \\
\hline$T C_{j}^{n c}$ & $\$ 50130$ & $\$ 57265$ & $\$ 91031$ & $\$ 37858$ & $\$ 236284$ \\
\hline
\end{tabular}




\begin{tabular}{|l|l|l|l|l|l|}
\hline$T C_{j r}^{n c}$ & $\$ 62157$ & $\$ 71038$ & $\$ 113104$ & $\$ 46886$ & $\$ 293185$ \\
\hline
\end{tabular}

Table 6.3 The optimal policy of distributors and pharmacies in the cooperative strategy

\begin{tabular}{|c|c|c|c|c|c|}
\hline$j$ & 1 & 2 & 3 & 4 & Sum \\
\hline$n_{j}^{c^{*}}$ & 1 & 1 & 1 & 1 & \\
\hline$t_{r}^{c^{*}}$ & 0.0978 & 0.085 & 0.054 & 0.129 & \\
\hline$t_{j}^{c^{*}}$ & 0.0978 & 0.085 & 0.054 & 0.129 & \\
\hline$q_{r}^{c^{*}}$ & 23543 & 27097.3 & 44055 & 17487 \\
\hline$Q_{j}^{1, c^{*}}$ & 0 & 0 & 0 & 0 & \\
\hline$q_{r}^{c^{*}} / t_{r}^{c^{*}}$ & 240704 & 315794 & 811208 & 135548 & 1503254 \\
\hline$\left(Q_{j}^{1, c^{*}}+q_{r}^{c^{*}}\right) / t_{j}^{c^{*}}$ & 240704 & 315794 & 811208 & 135548 & 1503254 \\
\hline$T C_{r}^{c}$ & $\$ 12169$ & $\$ 13950$ & $\$ 22417$ & $\$ 9116$ & $\$ 57652$ \\
\hline$T C_{j}^{c}$ & $\$ 7794$ & $\$ 8849$ & $\$ 13802$ & $\$ 5966$ & $\$ 36411$ \\
\hline$T C_{j r}^{c}$ & $\$ 19963$ & $\$ 22799$ & $\$ 36219$ & $\$ 15082$ & $\$ 94063.9$ \\
\hline
\end{tabular}

Table 6.4 The results of the model for the considered case study

\begin{tabular}{|c|c|c|c|}
\hline Variable & Value & Variable & Value \\
\hline$W_{G}^{n c}$ & $\$ 6439260$ & $\pi_{r}^{c}$ & $\$ 1467203.7$ \\
\hline$W_{G}^{c}$ & $\$ 3435946$ & $W_{r}^{c}$ & $\$ 4784005.89$ \\
\hline$\pi_{l m}^{n c}$ & $\$ 84276$ & $\pi_{j}^{c}$ & $\$ 638883.98$ \\
\hline$\pi_{m m}^{n c}$ & $\$ 148907.9$ & $W_{j}^{c}$ & $\$ 4087469.9$ \\
\hline$\pi_{l m}^{c}$ & $\$ 777761.56$ & $r$ & 0.2 \\
\hline$W_{l m}^{c}$ & $\$ 3273297.34$ & $p_{1}$ & 1.673 \\
\hline$\pi_{m m}^{c}$ & $\$ 842255.24$ & $p_{2}$ & 3.3724 \\
\hline$\pi_{r}^{n c}$ & $\$ 1087849$ & $p_{c}$ & 2.695 \\
\hline$W_{r}^{n c}$ & $\$ 6487750$ & $A_{c}$ & 1.5464 \\
\hline$\pi_{j}^{n c}$ & $\$ 140045$ & $d s_{c}$ & 0.1666 \\
\hline$W_{j}^{n c}$ & $\$ 6290804$ & $p s_{c}$ & 0.325 \\
\hline
\end{tabular}


To examine the effect of the parameters on the model results, a sensitivity analysis is completed. Three parameters, namely $C_{j}^{n c}, s$, and $R O C_{l m}$ are evaluated in this regard. Moreover, we mean a change of $+100 x \%$ on a parameter where the parameter is multiplied by the coefficient $(1+x)$, while $-100 x \%$ denotes the multiplication of a parameter by (1-x) coefficient.

\subsubsection{The ordering cost of the distributor}

As was shown, in the case of domestic production, the ordering cost of the distributor decreases, and this reduction has remarkable savings in the supply chain costs and deterioration of drugs. Therefore, we first examine the effects of this parameter on the deterioration cost and supply chain cost, and then show how the variation of the parameter affects the profitability of partners in the PSC.

The total deterioration cost of the supply chain versus the distributors' ordering cost has been depicted in Figure 6.2. The figure clearly shows that any progress in the reduction of ordering costs decreases the deterioration cost of the supply chain. Therefore, the formation of an alliance between the local and main manufacturers is an innovation, which empowered the supply chain against the high deterioration cost.

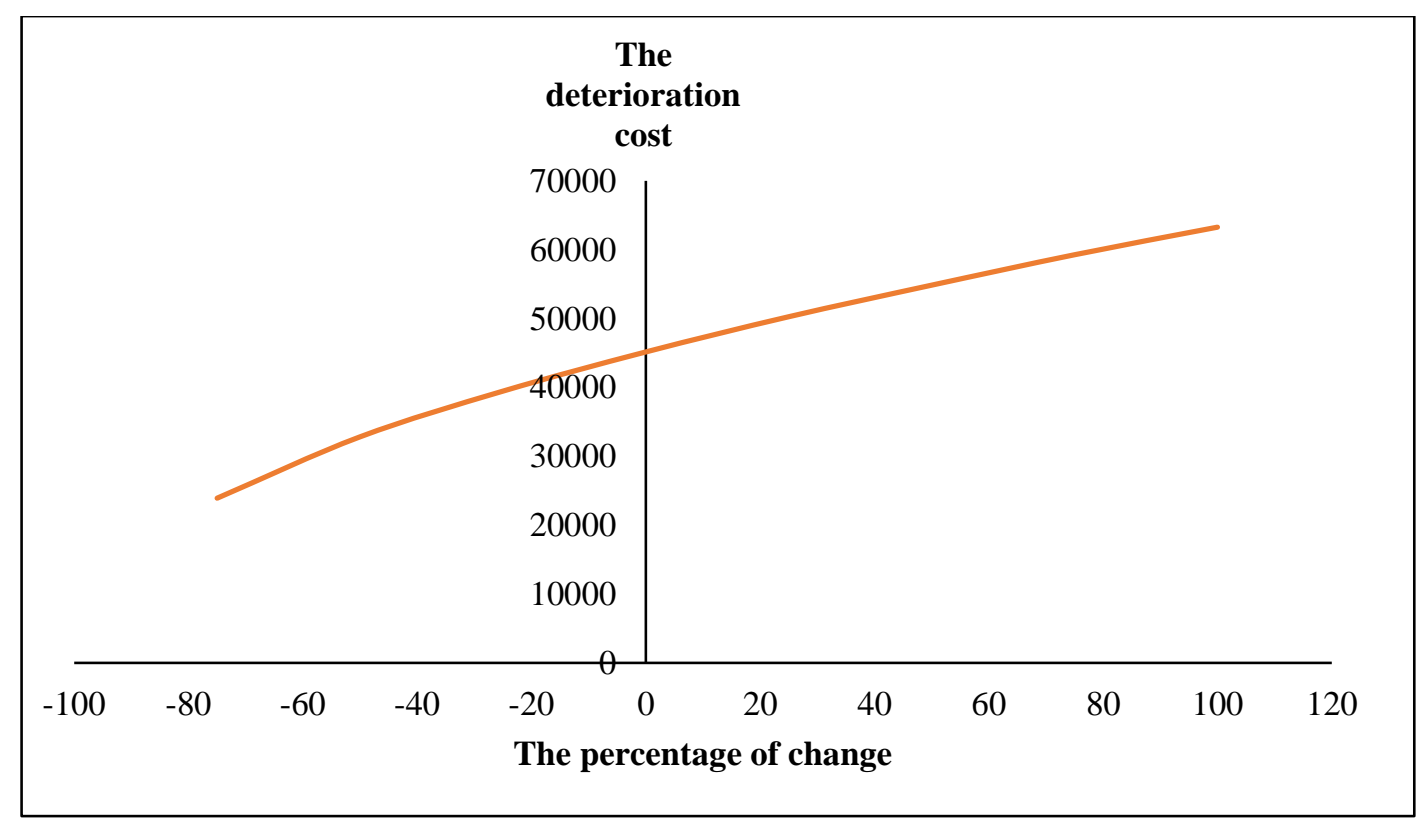

Figure 6.2 The deterioration cost vs. the ordering cost of distributors in the non-cooperative strategy 
As observed, changes in the ordering cost affect the deterioration quantity and cost. These lead to changes in the cooperative price and the cooperative gain of partners. In Table 6.5, the cooperative strategy results against the ordering cost of distributors in the non-cooperative strategy are presented. The results indicate that the higher the ordering costs of distributors, the greater the cooperative profitability of PSC members; also, there are more opportunities for participation. The results also indicate that as the ordering cost increases, the profit margin decreases further, resulting in lower patient and government costs.

Table 6.5 The results of the cooperative model against the non-cooperative ordering cost of the distributor

\begin{tabular}{|c|c|c|c|c|c|c|c|}
\hline $\begin{array}{c}\text { The percentage } \\
\text { of variation }\end{array}$ & $-50 \%$ & $-25 \%$ & 0 & $+25 \%$ & $+50 \%$ & $+75 \%$ & $+100 \%$ \\
\hline$p_{c}$ & 2.67 & 2.684 & 2.6948 & 2.705 & 2.714 & 2.722 & 2.73 \\
\hline$A_{c}$ & 1.567 & 1.556 & 1.5464 & 1.539 & 1.531 & 1.525 & 1.519 \\
\hline$A_{c} \times p_{c}$ & 4.183 & 4.17630 & 4.167 & 4.16299 & 4.15513 & 4.15105 & 4.14687 \\
\hline$P_{G}$ & 1106266 & 1116893 & 1125732 & 1133438 & 1140310 & 1146615 & 1152402 \\
\hline$P_{l m}$ & 675092.1 & 685089. & 693485. & 700865. & 707494. & 713617. & 719270. \\
\hline$P_{d}$ & 479811.1 & 490120 & 498838 & 506539. & 513484. & 519921. & 525883 \\
\hline$P_{r}$ & 372316 & 376160. & 379354 & 382144. & 384638 & 386932. & 389044 \\
\hline
\end{tabular}

\subsubsection{The market shares of the main manufacturer before cooperation $(s)$}

In the reference-based pricing, it was declared that the medicines are categorized based on their therapeutic benefits, and the price of medicines in a category will be almost the same, thus the price can be used as a reference for new similar drugs. Thus, the considered drugs could be regarded in the category of thalassemia improvement drugs, and the investigation about the market share of the drugs before forming the domestic licensee production could provide managerial insight about the selection of available brands in the cooperative strategy. However, it should be noted that the investigation here is just about the economic consideration of the brand, and the therapeutic benefits of one drug over other medicines may impair the assumption that medicines are homogeneous in one group.

In Figure 6.3, the cooperative profit of the local manufacturer versus the market share of the main manufacturer is shown. 


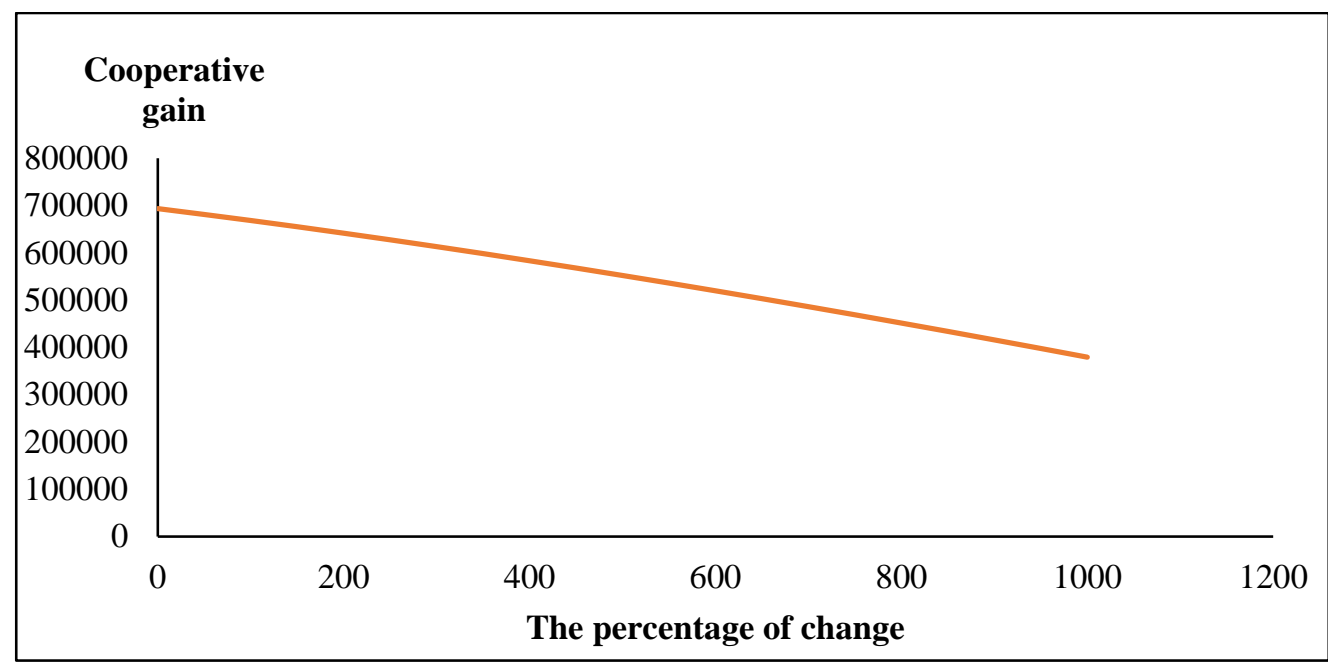

Figure 6.3 The cooperative gain of local manufacturer vs. the market shares of the main manufacturer

Also, Table 6.6 displays the results of the cooperative strategy for different market shares of the main manufacturer.

Table 6.6 The results of the cooperative model against the market shares of the main manufacturer

\begin{tabular}{|c|c|c|c|c|c|c|}
\hline The percentage of variation & $0 \%$ & $+100 \%$ & $+200 \%$ & $+300 \%$ & $+400 \%$ & $+500 \%$ \\
\hline$p_{c}$ & 2.6948 & 2.785 & 2.872 & 2.958 & 3.042 & 3.123 \\
\hline$A_{c}$ & 1.5464 & 1.518 & 1.492 & 1.467 & 1.445 & 1.423 \\
\hline$A_{c} \times p_{c}$ & 4.167239 & 4.22763 & 4.285024 & 4.339386 & 4.39569 & 4.444029 \\
\hline$P_{G}$ & 1125732 & 1049170 & 975684 & 904977.9 & 836799.7 & 770931.9 \\
\hline$P_{l m}$ & 693485.5 & 668337.3 & 641482.3 & 613067.3 & 583218.6 & 552046.2 \\
\hline$P_{d}$ & 498838.2 & 468800 & 439318.2 & 410375.6 & 381955.1 & 354039.4 \\
\hline$P_{r}$ & 379354.5 & 364342.2 & 348568.6 & 332105.1 & 315013 & 297345.3 \\
\hline
\end{tabular}

The results of Table 6.6 and Figure 6.4 are noteworthy in the sense that, by relying on them, the value of license could be interpreted. In this manner, the larger the market share of the main manufacturer, the higher the value of its production license, and this, in turn, reduces the profitability of the local manufacturer and other domestic members of the PSC. As a result, as the market share of the main manufacturer in the local market increases, the cooperative price will be higher, the profit margins of distributors and pharmacies will be lower, and government spending will be higher. With these results in mind, choosing a main manufacturer with a smaller market 
share can be a more efficient choice from the viewpoint of the government and other members. However, this conclusion is apart from the effectiveness of the medicine in terms of the health improvement of patients and is only a cost-benefit analysis. This conclusion is also supported by other research, such as Lauton et al. (2018), that showed that generics suppliers in PSC are not always the best choice for reducing costs and risks.

\subsubsection{The $R O C$ rate of the local manufacturer}

The final investigation of the chapter is related to the $R O C$ rate of the local manufacturer. This rate may implicitly point to the government's ability to provide funding to support domestic production. For example, bonds with the lowest expected rate but low risk refer to when shareholders request a low rate of return. Hence, implementing the proposed model for different values of $R O C$ leads to such results as in Figure 6.4 and Table 6.7.

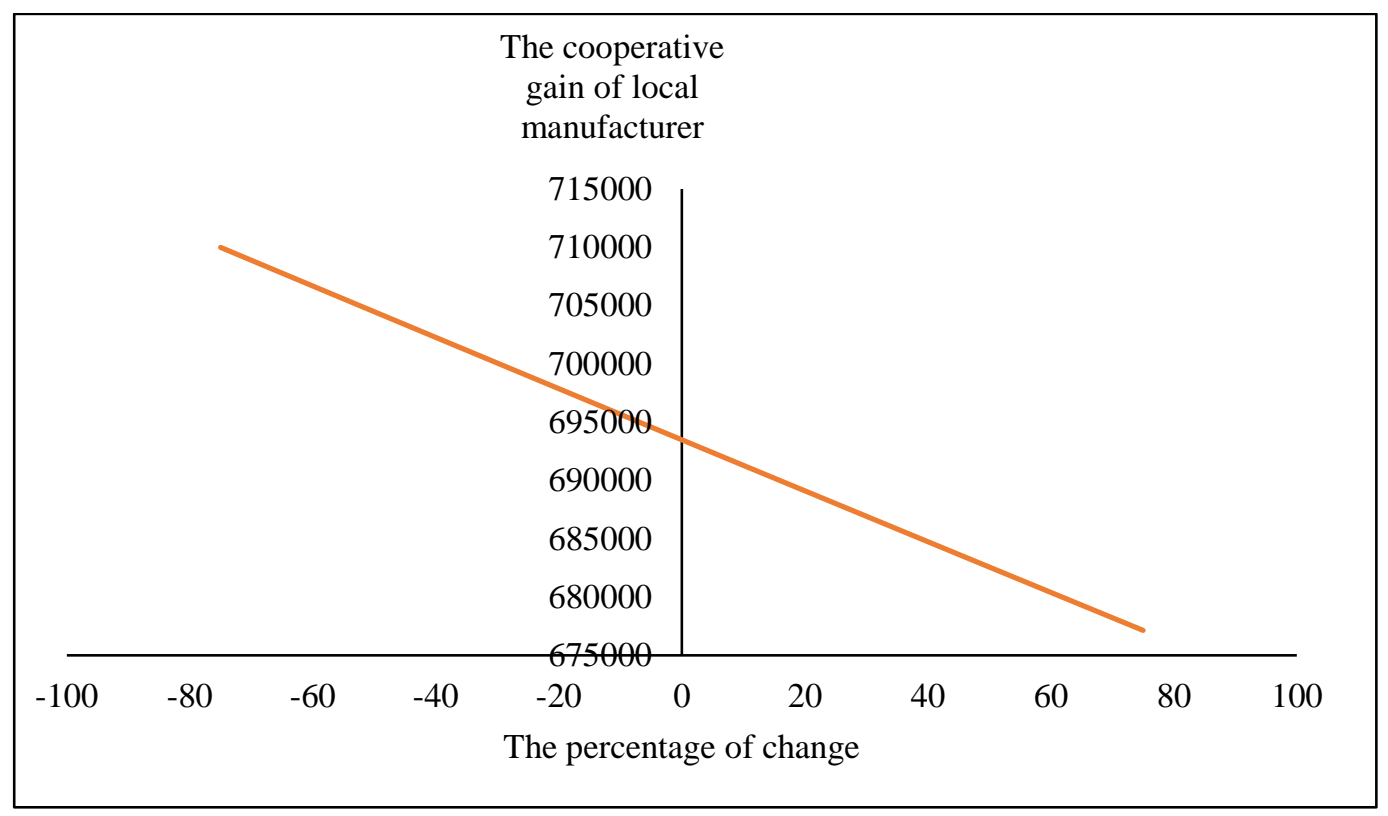

Figure 6.4 The cooperative gain of the local manufacturer vs. the market shares of the main manufacturer 
Table 6.7 The results of the cooperative model against the return on the capital rate of the main manufacturer

\begin{tabular}{|c|c|c|c|c|c|c|c|}
\hline The percentage of & $-75 \%$ & $-50 \%$ & $-25 \%$ & $0 \%$ & $+25 \%$ & $+50 \%$ & $+75 \%$ \\
\hline$p_{c}$ & 2.675 & 2.681 & 2.688 & 2.6948 & 2.702 & 2.708 & 2.715 \\
\hline$A_{c}$ & 1.553 & 1.551 & 1.549 & 1.5464 & 1.544 & 1.542 & 1.54 \\
\hline$A_{c} \times p_{c}$ & 4.15427 & 4.15823 & 4.16371 & 4.16723 & 4.17188 & 4.17573 & 4.1811 \\
\hline$P_{G}$ & $11 \tilde{4301}$ & 113726 & 113150 & 112573 & $11 \hat{1994}$ & 111414 & 110833 \\
\hline$P_{l m}$ & 709989. & 704470 & 698969. & $693 \hat{4} 85$. & 688019. & 682571 & 677141. \\
\hline$P_{d}$ & 505518. & $503 \hat{301 .}$ & 501074. & $498 \overline{838}$. & $496 \overline{59} 92$. & 494338. & 492074. \\
\hline$P_{r}$ & 382545 & $38 \hat{1492}$ & $380 \hat{4} 28$. & 379354. & 378269. & $377 \hat{174}$. & $37 \hat{6068}$ \\
\hline
\end{tabular}

According to Figure 6.4 and Table 6.7, the higher power of the local manufacturer in its $R O C$ rate causes fewer benefits for other members of PSC, such as the government, distributors, and pharmacies. The increases also reduce the utility of patents because they will incur more medical expenses. Based on the results, the government is more inclined to grant exclusive production licenses to firms with lower $R O C$ rates. For example, semi-state-owned companies that can finance by issuing securities have a higher priority for the exclusive production of drugs.

However, this should be noted that the analysis here is just a cost-benefit analysis and more explanatory research is needed to examine all aspects that are important in partner selection. For example, when corporate innovation is the matter of joint R\&D investment by pharmaceutical manufacturers in local countries, the effectiveness of local contracting institutions and their preliminary readiness for adopting joint $R \& D$ activities have a lasting effect on promoting corporate innovation (Sun et al., 2018). Also, Bagherzadeh et al. (2019) discussed that managers who aimed to establish a collaboration strategy and boost innovation performance should prepare the company internally by developing an innovation strategy and internal and external knowledge sharing processes.

\subsection{Research and Managerial Implications}

The chapter's research implications and managerial insights are retrieved from the model itself and the model implementation results. The proposed model examined the effectiveness of a form of strategic alliance and its benefits for the pharmaceutical supply chains. It clearly focused on the 
deterioration of pharmaceutical products as an important challenge of PSC. Meanwhile, the formation of alliances by domestic production of patented drugs is shown to alleviate this challenge. In this regard, the main effect of the strategy is to decrease the ordering cost of distributors significantly because there is no need to import drugs anymore. This, in turn, reduces the deterioration amounts as the results indicated. Moreover, alongside the advantages of the strategy in reducing costs of PSC, the mechanism considers some portion of the generated surplus profit for the government due to its direct influence on granting the exclusive production right to the patented medicine. Therefore, the considered strategy not only benefits the direct members of PSC, but also provides utilities for the patents as a result of decreasing the cost and reducing the exorbitant cost of the government in the health care system. This conclusion suggests the government advocates the thesis's cooperative strategy and facilitates the formation of the strategic alliance.

As explained, facilitating international cooperation of manufacturers through license agreements can be one of the most effective ways to reduce the deterioration of pharmaceutical products as one of the main challenges in this field. This strategy, alongside the supply chain management practice, could lead to outcomes that not only result in significant cost savings, but also in the reduction of the deterioration which can be many times greater than conventional solutions such as preservation technologies, which themselves require a significant amount of investment. Given the theoretical foundations of the research, the reason for this would be obvious: reducing the length of ordering periods of imported drugs, as some research has recommended the long period of importing drugs as an indicator for the low level of service of the supply chain. On the other hand, the present study provides managers with a systematic approach to evaluate the strategy, such as a theoretical framework for supporting price modification as an innovative scheme in the reference-based pricing mechanism and the fair distribution of profits among members based on common mechanisms in reality. So, managers could rely on the framework of the chapter to provide the necessary motivation and coordination between members in addition to their own positive and supportive view of the strategy that will lead stockholders to support the strategy.

Other insights are stemming from the numerical results and sensitivity analysis. The first insight of the numerical results is about the outstanding role of the ordering costs in the deteriorationrelated costs. Moreover, the market shares of the main manufacturer before establishing a local licensor manufacturer are regarded as another determinant parameter of the model. The 
investigation showed that a higher market share of the main manufacturer leads to a higher valuation for licenses and production rights, which sequentially increases the cooperative price and reduces the profitability of members in the cooperative strategy. Management insight in this respect is that if there are several options for external participation, the less-known manufacturers with fewer market shares are better options than well-known manufacturers, although the risk of working with these manufacturers is higher. A similar analysis was investigated by local manufacturers. In this regard, it was observed that local manufacturers with fewer $R O C$ rates would bring lower cooperative prices and higher profits for the PSC. Therefore, to finance the construction of domestic manufacturing companies, semi-state-owned companies or companies with expected fewer interest rates can bring more profits for the supply chain.

\subsection{Conclusions}

In this chapter, the profit split problem between members of a PSC, including government, a local manufacturer, distributors, and pharmacies, resulted from applying a cooperative strategy, i.e., granting the exclusive production right to a local licensed manufacturer, was studied. The benefits of this strategy were outlined as reducing the ordering cost of distributors, reducing the cost of deterioration in PSC, reducing the cost of medicines and the convenience of patients, and increasing the profitability of members. For this purpose, after modeling the profit and cost functions of members in the cooperative and non-cooperative strategies, two profit split mechanisms were used. The first mechanism was implemented between the main and local manufacturers and used a proportional contract based on the cooperative game theory. This mechanism was used because measuring the performance of manufacturers in both cooperative and non-cooperative strategies as possible. Another mechanism distributes the profit of a cooperative strategy between the government, the distributors, the pharmacies, and the licensee.

The profit split was completed by determining the price and profit margin after implementing the cooperative strategy. In this case, it was explained that due to the inability to estimate the value of the members' coalitions, it is not possible to use the cooperative game theory. However, the costbased profit split mechanism also provides a good split in line with many real-world issues. The necessary and sufficient conditions for the profitability of the cooperative strategy were expressed as well. 
The chapter has several contributions to the literature. First, to value intellectual property, emphasize the capabilities of the patent holder, the efforts of other members in converting the asset to profits could be considered by the proposed model. Such an approach not only better reflects the value of the asset, but also motivates members to cooperate. These efforts included reducing the deterioration due to better supply chain policies in the cooperative strategy, and exercising legal authority to influence the supply chain, such as the government granting the exclusive production right. Also, new pricing mechanisms and price correction methods to distribute the revenue of the cooperation strategy were other research contributions that can be regarded as a supply chain coordination mechanism. As mentioned in the literature, slight changes in common pricing of pharmaceutical products are referred to as innovative pharmaceutical pricing agreements. Therefore, the proposed pricing mechanism is based on real-world practices, is another contribution. Finally, the effectiveness of the proposed collaboration strategy was confirmed both in theory and using numerical results. The results not only encourage the pharmaceutical supply chain to adopt the strategy, but also guide for selecting among pharmaceutical brands through license agreements. The growth of such alliances may be evidence of the effectiveness of the strategy under study.

The model presented was applied to a case study for producing domestic production of a brand drug for thalassemia patients. The results of this evaluation showed that, as expected, the cooperative strategy provides significant profits for all members of the PSC. The stemmed royalty rate, based on the proposed model, was close to the actual implemented values, and the proposed framework was introduced as a complement to current pricing mechanisms. Moreover, the sensitivity analysis was carried out to explain the behavior of the model against some parameters. The sensitivity analysis confirmed the validity of the model and offered managerial insights for conducting the pharmaceutical-related business, as explained in the previous section. 


\section{Chapter 7: Conclusions and future research}

In the previous chapters, the selected problems in the context of PSC were presented. The main objectives considered in this thesis were described as pricing decisions of pharmaceutical products, cooperative strategies by the participation of PSC members, and the positive effects they have on reducing transaction costs, ordering costs, deterioration costs as well as total health care costs. Regarding different assumptions, four cases were studied in the thesis, and for each case, the profits of partners before and after the cooperation were modeled to determine the benefits of the cooperation and decide about assigning the benefits to the partners. Also, the proposed models were implemented in real case studies, and the results of implementations, as well as the managerial insights of the thesis, were presented.

In this chapter, the concluding remarks of the thesis, answers to the thesis questions, and future study directions are provided.

\subsection{Conclusions}

In this thesis, four cooperative strategies in the pharmaceutical supply chain were studied. The strategies, concluding remarks and insights of the strategy are summarized as follows:

1) Chapter 3 discussed the general license contract between the local and main manufacturer, the ownership structure of the cooperative alliance, and the way the operational decisions, such as pricing decisions, could affect the license contract parameters. Moreover, due to the fact that the licensor and licensee are often from different countries, and this yields to different tax rates for them, the manufacturers were distinguished by denoting them as local or foreign manufacturers. To distribute the benefits of cooperation and determine the parameters of the license contract between local and foreign manufacturers, the proportional contract was employed. Also, providing the necessary and sufficient conditions, an index called "the marginal free-tax operational gain on the share" was introduced that stipulated whether local manufacturer ownership or shared ownership of local and foreign manufacturers is optimal. The results of this chapter showed that the operational decisions of the alliance should be such that the outcome of the alliance as a whole is maximized, and the ownership structure and the license terms could not affect the optimal decisions in this manner. Furthermore, the ownership structure, which is 
influenced by the partners' specifications, such as stand-alone profit and imposed tax rates of partners, should be determined based on the capabilities of partners in converting the capital assets of the alliance into the resulting profit of the local market in which the cooperative strategy is performed. Sensitivity analysis on model parameters also reveals valuable management results and insights. In this regard, it was illustrated those new emerging licensors with a less expected rate of return on investment and financing the alliance at the lowest expected interest rates would eventually lead to lower market prices and greater social welfare for customers. The latter could provide guidelines for the government in granting the exclusive production rights and financing the public and social healthcare concern projects.

2) The proposed models of chapter 3 were extended in chapter 4 to study a more complex environment in the context of the pharmaceutical supply chain and provide a more accurate valuation method. This chapter, alongside chapter 3, addressed the evaluation of the cooperative strategies in the PSC for medicines with competitive pricing mechanisms. However, the oligopoly market structure in chapter 4 has more complexity than that of chapter 3 in the sense that it needed to solve a second-level mathematical programming model to extract the equilibrium price. The uncertainty about future demand, the depreciation consideration, the liquidation option for the alliance in the middle periods of the planning horizon, and the capacity planning and financing decisions were issues added to the valuing method of chapter 3 to prepare a more realistic and comprehensive method. For these purposes, a stochastic programming model was proposed that aimed to maximize the gain of partners in the planning horizon according to the proportional license contract. Regarding the parameters of the license contract, it was shown that the proposed model is a non-linear mixed-integer programming model. Hence, we borrowed the concept of "the marginal free-tax operational gain on the share" and the ideal gain functions of partners to propose an innovative dynamic programming method to extract the optimal solution of the model, especially for large-size problems. The proposed models and algorithms were implemented to value an alliance for manufacturing Citric acid under the license of a selected foreign firm. The results of the chapter emphasized the efficiency of the proposed algorithm in terms of the solving time and optimality of solutions. The license contract parameters and the value of cooperative alliance have also been shown to be not far from 
the existing valuing methods though it takes into account many parameters about the capabilities and specifications of the involved partners. The results of the sensitivity analysis on the proposed models in this chapter also implied the results in line and similar to the previous chapter.

3) Chapters 5 and 6 investigated pharmaceutical products. Their pricing mechanism was mainly under the control of the government and under the supervision of other legislatures. The patented medicines in this context are known as branded medicines, for which the right to license in exchange for obtaining the production and distribution rights is common in many countries. The reference-based pricing mechanism was adopted for the medicines due to the case study of this chapter, although this mechanism is prevalent in many other countries. It was also explained that, by this arrangement, the main and local manufacturer could develop their market and reduce their production and distribution costs. Due to the important role of the government in granting the production rights exclusively to the intended alliance, the government was introduced as a player that could expect some benefits from the alliance. Hence in chapter 5, the problems of modifying the market price and distributing the profit of cooperation between involved members were scrutinized. To do so, cooperative game theory and the Nash bargaining solution were applied in two directions. The first direction examined the game theory to determine the license contract parameters between the local and main manufacturers. In the second direction, a negotiation game was performed between the cooperative alliance of the manufacturer and the government to modify the price of medicines as discounts granted to the health care system in exchange for exclusive production rights to the alliance. The order of mentioned games was such that knowing the license contract parameters of manufacturers as a function of ongoing price, which was identified using the Nash bargaining model, the Nash bargaining solution was applied again on the second game between manufacturers and the government to decide about the new price. In this problem, through numerical case studies, it was demonstrated that the market share of the licensor in the local market before the cooperation, the existing price of the brand medicine in the local market, the financing capabilities of the local and main manufacturers, the predetermined marginal profit of pharmacies and distributors in the logistic network, and the tax rate on the income of 
partners are parameters that affect the profit split and the pricing and formation decisions in this cooperative strategy.

4) The final strategy of the thesis was the development of models in chapter 6 into new models that take into account the benefits of the cooperative strategy in terms of the reduction in ordering and deterioration-related costs of distributors and pharmacies as one of the main concerns of the PSC. In this problem, the proportional contract was adopted again to adjust the parameters of the license contract. In the PSC understudy, the distributor-managed inventory policy was used to serve pharmacies by distributors. This policy was modeled to measure the costs of pharmacies and distributors. The proposed model demonstrated the importance of the deterioration and ordering costs on the order quantity decisions of pharmacies and distributors. Therefore, the proposed cooperative strategy that reduces these costs could improve the efficiency of partners who had no direct role in the production process. Moreover, by identifying the profit of partners before and after the cooperative strategies, the resulting surplus of cooperation was distributed between partners proportional to their incurred costs of the PSC under investigation. For this purpose, the license contract parameters of the manufacturer, the new price of brand medicines as well as the profit margins of pharmacies and distributors were modified such that the surplus profit is split between partners fairly according to the mentioned mechanism. The proposed models in this chapter were implemented in a case study that was about the under-license production of Jadenu ${ }^{\circledR}$, an efficient brand medicine for the treatment of Thalassemia patients, by a local manufacturer in Iran. The results of implementation exhibited by the main manufacturer's market share, negatively affected the price of the brand in the local market and the profitability of the partners, although financing using semi-state-owned companies for government showed that leads to more desires for the government in reducing the costs of patients. Just-in-time production, i.e., the production just equal to the amount required, as the result of lowering the ordering costs of pharmacies and distributors, was introduced as the main advantage of the proposed strategy that could alleviate deterioration as one of the main concerns of the pharmaceutical supply chain. 
5) For all proposed models, optimality conditions and proper algorithms were presented to extract optimal solutions. The solutions were also compared with the current solution in the PSC to validate the models and the efficiency of the proposed strategies.

\subsection{Significant contribution from current COVID-19 perspective}

The significance of this thesis and its contributions has become much bolder since the outbreak of Covid -19. Providing reliable, fast, and cost-effective health services is of great importance in the health systems of communities. In this context, the pharmaceutical supply chain can play an important role in achieving health-related goals by providing the right amount of medicines at the right time. Recently, vaccine supply has become the priority of every government to protect its population against this brutal humankind enemy.

The distribution of vaccines among all affected countries has become one of the most challenging issues of this supply chain. Currently, due to the limited production capacity, all the efforts have been inequitable and inefficient. Although supplies of vaccines are already growing but still not enough to meet this huge demand by the world. Death rates have been high and the situation is getting worse in some poor and middle-income countries like Iran. Because of the insufficient supply of the vaccine, most of the most vulnerable unvaccinated people look unlikely to be vaccinated soon.

In this thesis, we implement our proposed model for a specific drug that is patent-based. The vaccine supply chain has its specific players and specifications, but intellectual property is the essence of this supply chain as well. As a matter of fact that the vaccine production is patent-based so the expansion of thesis contribution in this context is worth mentioning.

Many experts argue that millions of doses might become available if patent owners shared their secrets. Unfortunately, the patent waiver is just an empty gesture as its effects in long term are unpredictable. Apart from disrupting supply chains, it will ultimately cause deterring innovations. Intellectual property rights play a vital role in the pharmaceutical industry due to the high costs of the $R \& D$ process. This issue is much more important in the vaccine supply chain these days as adequate vaccine supply not only saves lives and decreasing mortality rates but also recovers the economy. So in the vaccine supply chain, we can get the most of strategic alliances. Although establishing such alliances takes time and can not recreate in a hurry, but it will be the best solution 
in the long term. In doing so, putting the idea into the context of long-term strategies such as coordinated approaches to the sharing of intellectual property and technology transfer should be a win-win solution that is studied in this thesis carefully.

First, to value intellectual property, in addition to the capabilities of the patent holder, the efforts of other supply chain members in converting the asset to profits could be taken into account by the proposed model. Such an approach not only better reflects the value of the asset but also motivates members to cooperate. However, this thesis presents a suitable framework for evaluating and implementing the strategy.

An initiative strategy in the pharmaceutical supply chain with the participation of production technology providing firms increases the social welfare and sustainability aspects besides efficiency of the supply chain. In this regard, it should be noted that the goals of health care systems are beyond the self-interest of the members, and they are committed to protecting human beings as much as they can. Considering a cooperative strategy, which also is encouraged by the government, contributes to social welfare by providing cheaper medicines and in a faster manner

for patients. This strategy improves the agility of the supply chain and decreases the cost of medicines by reductions in the cost of providing such medicines. In addition, this strategy increases the market share of the considered drugs.

\subsection{Answer to research questions}

In this section, the questions of the thesis are mentioned, and the answers to them based on the thesis conclusion are provided. The questions and their answers are as follows:

1. How can the new design of the supply chain with a smooth and proper strategy result in increasing the performance of the pharmaceutical supply chain? Does this new design provide the customer with the same medicine at a lower price and less time, resulting in better customer satisfaction?

\section{Answer:}

In all cooperative strategies presented in chapters 4-6, it was shown that the price of domestically produced medicines is less than the imported ones supplied in the local market 
before the establishment of the cooperative alliance. For the cooperative strategy in chapter 4, Proposition 4.1 proved this fact, and the surplus regarded by the government in the strategies of chapters 5 and 6 , clearly reflected the reduction of brand medicines manufactured domestically under the license contract.

2. How can we measure the value of a cooperative alliance and determine the share of profits for the various partners involved in the intended alliance? Does the proposed cooperative alliance provide a "win-win" situation for the players?

Answer: The models introduced in chapters 3 and 4 addressed this question. For this purpose, valuation methods based on the presented value of future cash flows were utilized. Accordingly, the cash flows of partners in the alliance should be calculated in this method, and these cash flows depended on the license income and ownership structure of the alliance. Therefore, alongside the valuation method, the proportional contract with the aim of maximizing the surplus of the cooperation, which was split equally between the partners, was explored. The proposed model of the thesis, which was designed to address other strategic and operational decisions in addition to the valuing purposes, was categorized as the Free Cash Flow to Equity (FCFE) valuation method. The valuing method added depreciation to the partner's share of net income and reduced the interest, tax, and principles of loan from it. The proposed strategies had advantages such as reducing transaction costs, reducing production costs, and taking advantage of the monopoly market. Furthermore, the proportional contract was described as a contract that provides a win-win solution by distributing the surplus of cooperation between partners equally.

3. How can we control and reduce the deterioration rate of pharmaceutical products, and how beneficial are these developed models for customers?

Answer: The cooperative strategy proposed in chapter 6 evaluated this question. Indeed, the production of licensed medicines in the local country significantly reduces the ordering costs in the PSC network. This consequently reduces the ordering quantity throughout the network and makes possible a production and distribution system almost similar to the justin-time philosophy. This change ultimately reduces the deterioration of medicines and 
enhances the capabilities of the PSC for handling medicines that are under a remarkable amount of deterioration.

\subsection{Future research}

The future works stemming from this dissertation could be as follows:

1) In this thesis, the value of control in the JV was not studied, whereas this issue has a significant role in joint venture outputs and partners' intentions. To consider the effect of control, one can tie the demand function as a function of the partner's share that reflects the level of their control in the JV and affects the quality of JV outputs.

2) The local partner or the smaller partner usually harbors some aims to form an alliance with stronger partners beyond just the resulting profit of the cooperation. They might pursue to reach the knowledge about the technology of other partners and might declare its purpose explicitly or implicitly. When it is declared explicitly, the technology-providing partner could ask for more profit in the JV to help other partners, for example, by considering the cost of learning that should be paid by the local partner. Also, in the case that a technologyproviding partner aims to protect his technology, some restrictions on the share of the JV and access of the local partners to the codified knowledge or license should be imposed by the technology partners. These restrictions should be expressed both in the JV agreement and implementation.

3) Partner selection and task allocation to them in the $J V$ is an important topic. In Hsueh and Yan (2011), the Shapley value was used to address this problem. The results of Hsueh and Yan (2010), alongside the models proposed in this chapter, and the sequential bargaining models can be integrated to provide a useful framework for firms that attempt to find desired partners and do business with them jointly.

4) Considering other market structures for the alliance, such as the oligopoly market of nonhomogeneous pharmaceutical products or under-control markets, are valuable issues for future studies. 
5) Extending the deterministic model of the thesis in chapters 5 and 6 to an uncertain model is another suggestion for future studies, which could disclose some facts about the uncertain environment of PSC and could be examined in future research.

6) It is also proposed to provide a new model for the cooperative alliance of medicines that is still in the early stages of testing and auditing, and the investment in them entails tackling more uncertainty and risks. 


\section{REFERENCES:}

Abbott, F. M. (2002). The TRIPs agreement, access to medicines, and the WTO Doha ministerial conference. The journal of world intellectual property, 5(1), 15-52.

Abdulghani, I., Bermamet, H., \& Altarazi, S. (2019, April). Pharmaceutical Supply Chain Cost Optimization Model Considering Multiple Echelons and Multiple Medicines. In 2019 IEEE 6th International Conference on Industrial Engineering and Applications (ICIEA) (pp. 709-713). IEEE.

Adegbesan, J. A. (2009). On the origins of competitive advantage: Strategic factor markets and heterogeneous resource complementarity. Academy of Management Review, 34(3), 463475.

Adegbesan, J. A., and Higgins, M. J. (2011). The intra-alliance division of value created through collaboration. Strategic Management Journal, 32(2), 187-211.

Alam, A., Uddin, M., and Yazdifar, H. (2019). Financing behaviour of R\&D investment in the emerging markets: the role of alliance and financial system. $R \& D$ Management, 49(1), 21 32.

Al-Emadi, T. (2010). Joint Venture Contracts (JVCs) among Current Negotiated Petroleum Contracts: A Literature Review of JVCs Development, Concept and Elements. Georgetown Journal of International Law, 1, 645.

Alletto, A., Bruccoleri, M., Mazzola, E., \& Ramanathan, U. (2017). Collaboration experience in the supply chain of knowledge and patent development. Production Planning \& Control, 28(6-8), 574-586.

Alves, H., Ferreira, J. J., and Fernandes, C. I. (2016). Customer's operant resources effects on cocreation activities. Journal of Innovation and Knowledge, 1(2), 69-80.

Arana, K., Flores, K., Ramos, E., \& Mesia, R. (2020, July). Developing a Two-Echelon Inventory Framework in Pharmaceutical Supply Chain: An Empirical Review. In International Conference on Applied Human Factors and Ergonomics (pp. 361-368). Springer, Cham.

Azzi, A., et al. (2013). "Medicine inventory management and distribution: outsourcing logistics to third-party providers." Strategic Outsourcing: An International Journal 6(1): 48-64.

Baboli, A., Fondrevelle, J., Tavakkoli-Moghaddam, R., \& Mehrabi, A. (2011). A replenishment policy based on joint optimization in a downstream pharmaceutical supply chain: centralized vs. decentralized replenishment. The International Journal of Advanced Manufacturing Technology, 57(1), 367-378. 
Baetens, F. (2009). Human Rights and the WTO: The Case of Patents and Access to Medicines by HOLGER HESTERMEYER [Oxford University Press, Oxford, 2007, 369 pp, ISBN 978 0-19-955217-7 (p/bk)]. International \& Comparative Law Quarterly, 58(3), 747-748.

Bagherzadeh, M., Markovic, S., Cheng, J., \& Vanhaverbeke, W. (2019). How does outside-in open innovation influence innovation performance? Analyzing the mediating roles of knowledge sharing and innovation strategy. IEEE Transactions on Engineering Management, 67(3), 740-753.

Balcázar-Camacho, D. A., et al. (2016). "Strategic guidelines for supply chain coordination in healthcare and a mathematical model as a proposed mechanism for the measurement of coordination effects." Dyna 83(197): 203-211.

Baldick, R., Grant, R., \& Kahn, E. (2004). Theory and application of linear supply function equilibrium in electricity markets. Journal of regulatory economics, 25(2), 143-167.

Barquin, J., Centeno, E., \& Reneses, J. (2004). Medium-term generation programming in competitive environments: a new optimisation approach for market equilibrium computing. I.E.E. Proceedings-Generation, Transmission and Distribution, 151(1), 119126.

Belderbos, R., Cassiman, B., Faems, D., Leten, B., and Van Looy, B. (2014). Co-ownership of intellectual property: Exploring the value-appropriation and value-creation implications of co-patenting with different partners. Research policy, 43(5), 841-852.

Bengtsson, M., and Kock, S. (2014). Coopetition-Quo vadis? Past accomplishments and future challenges. Industrial marketing management, 43(2), 180-188.

Bhagwat, Y. and F. Griggs (1995). "Analysis of riskiness of pharmaceutical industry firms." Journal of Research in Pharmaceutical Economics 6(1): 65-76.

Booth, R. (1999). "The global supply chain. FT healthcare management report." London: Financial Times Business Ltd.

Bouncken, R. B., Gast, J., Kraus, S., and Bogers, M. (2015). Coopetition: a systematic review, synthesis, and future research directions. Review of Managerial Science, 9(3), 577-601.

Cabral, L., \& Pacheco-de-Almeida, G. (2019). Alliance formation and firm value. Management science, 65(2), 879-895.

Cachon, G. P. (2004). "Supply chain coordination with contracts." Handbooks in operations research and management science 11: 227-339.

Cachon, G. P., and Lariviere, M. A. (2005). Supply chain coordination with revenue-sharing contracts: strengths and limitations. Management Science, 51(1), 30-44.

Chen, X., Yang, H., and Wang, X. (2019). Effects of price cap regulation on the pharmaceutical supply chain. Journal of Business Research, 97, 281-290. 
Child, J., Faulkner, D., and Tallman, S. B. (2005). Cooperative strategy. Oxford University Press, USA.

Ching, A. T. (2010). A dynamic oligopoly structural model for the prescription medicine market after patent expiration. International Economic Review, 51(4), 1175-1207.

Coffey, K. C. (2013). Is there a role for expired medications in developing countries? Clinical pediatrics, 52(1), 7-9.

Contractor, F. J., and Woodley, J. A. (2015). How the alliance pie is split: Value appropriation by each partner in cross-border technology transfer alliances? Journal of World Business, 50(3), 535-547.

Csanádi, M., Kaló, Z., Prins, C. P., Grélinger, E., Kiss, A. M., Fricke, F. U., ... and Vokó, Z. (2018). The implications of external price referencing on pharmaceutical list prices in Europe. Health Policy and Technology, 7(3), 243-250.

Cummings, J., Reiber, C., \& Kumar, P. (2018). The price of progress: Funding and financing Alzheimer's disease drug development. Alzheimer's \& Dementia: Translational Research \& Clinical Interventions, 4, 330-343.

Dantzig, G. B., \& Infanger, G. (1995). A probabilistic lower bound for two-stage stochastic programs (No. DOE/ER/25116-T3; SOL-95-6). Stanford Univ., Dept. of Operations Research, CA (United States).

Day, C. J., Hobbs, B. F., \& Pang, J. S. (2002). Oligopolistic competition in power networks: a conjectured supply function approach. IEEE Transactions on power systems, 17(3), 597607.

de Haro, S. L., Martín, P. S., De la Hoz Ardiz, J. E., \& Caro, J. F. (2007). Estimating conjectural variations for electricity market models. European Journal of Operational Research, 181(3), 1322-1338.

de Morais, R. P. S. (2017). Compulsory licensing of pharmaceuticals by the developing south. Economics Letters, 161, 74-77.

Devece, C., Ribeiro-Soriano, D. E., and Palacios-Marqués, D. (2019). Coopetition as the new trend in inter-firm alliances: literature review and research patterns. Review of Managerial Science, 13(2), 207-226.

Dewandaru, G., Masih, R., Bacha, O. I., \& Masih, A. M. M. (2015). Combining momentum, value, and quality for the Islamic equity portfolio: Multi-style rotation strategies using augmented Black Litterman factor model. Pacific-Basin Finance Journal, 34, 205-232.

Díaz, C. A., Villar, J., Campos, F. A., \& Reneses, J. (2010). Electricity market equilibrium based on conjectural variations. Electric power systems research, 80(12), 1572-1579.

Drover, W., Busenitz, L., Matusik, S., Townsend, D., Anglin, A., and Dushnitsky, G. (2017). A review and road map of entrepreneurial equity financing research: venture capital, 
corporate venture capital, angel investment, crowdfunding, and accelerators. Journal of Management, 43(6), 1820-1853.

Drummond, M., and Sculpher, M. (2005). Common methodological flaws in economic evaluations. Medical care, II5-II14.

Duan, Y., Li, G., and Huo, J. (2014). Supply chain coordination for fixed lifetime products with permissible delay in payments. Mathematical Problems in Engineering, 2014.

Dunlop, W. C., Staufer, A., Levy, P., and Edwards, G. J. (2018). Innovative pharmaceutical pricing agreements in five European markets: A survey of stakeholder attitudes and experience. Health policy, 122(5), 528-532.

Forman, L. (2007). Trade rules, intellectual property, and the right to health. Ethics \& International Affairs, 21(3), 337-357.

Garattini, L., Curto, A., and Freemantle, N. (2016). Pharmaceutical price schemes in Europe: time for a 'continental'one? Pharmacoeconomics, 34(5), 423-426.

Garattini, L., Salvioni, F., Scopelliti, D., and Garattini, S. (1994). A comparative analysis of the pharmaceutical market in four European countries. Pharmacoeconomics, 6(5), 417-423.

Giannoccaro, I., and Pontrandolfo, P. (2004). Supply chain coordination by revenue sharing contracts. International Journal of Production Economics, 89(2), 131-139.

Girmscheid, G., and Brockmann, C. (2009). Inter-and intraorganizational trust in international construction joint ventures. Journal of Construction Engineering and Management, 136(3), 353-360.

Gnyawali, D. R., and Park, B. J. (2009). Co-opetition and technological innovation in small and medium-sized enterprises: A multilevel conceptual model. Journal of small business management, 47(3), 308-330.

Gnyawali, D. R., and Park, B. J. R. (2011). Co-opetition between giants: Collaboration with competitors for technological innovation. Research Policy, 40(5), 650-663.

Govindan, K., and Popiuc, M. N. (2014). Reverse supply chain coordination by revenue sharing contract: A case for the personal computers industry. European Journal of Operational Research, 233(2), 326-336.

Grabowski, H. (1997). "The effect of pharmacoeconomics on company research and development decisions." Pharmacoeconomics 11(5): 389-397.

Grossman, S. J., and Hart, O. D. (1986). The costs and benefits of ownership: A theory of vertical and lateral integration. Journal of political economy, 94(4), 691-719.

Hammami, R., and Frein, Y. (2015). Redesign of global supply chains with integration of transfer pricing: Mathematical modeling and managerial insights. International Journal of Production Economics, 158, 267-277. 
Hassan, T., et al. (2006). "Re-organizing the pharmaceutical supply chain downstream: implementation a new pharmacy." IFAC Proceedings Volumes 39(3): 727-732.

Hokkanen, J., Linnosmaa, I., and Siikanen, M. (2016). Long-run effects of the generic substitution policy on prices of branded and generic pharmaceuticals.

Hong, Y., and WM Chan, D. (2014). Research trend of joint ventures in construction: a two-decade taxonomic review. Journal of Facilities Management, 12(2), 118-141.

Hossain, I., \& Parvez, S. (2020). Investigating the Effect of Extended Vendor Managed Inventory in the Supply Chain of Health Care Sector to Enhance Information Exchange. International Journal of Information and Management Sciences, 31(2), 171-189.

Hosseini-Motlagh, S.-M., Govindan, K., Nematollahi, M., and Jokar, A. (2019). An adjustable bilevel wholesale price contract for coordinating a supply chain under scenario-based stochastic demand. International Journal of Production Economics, 214, 175-195.

Hosseini-Motlagh, S.-M., Nematollahi, M., Johari, M., and Sarker, B. R. (2018). A collaborative model for coordination of monopolistic manufacturer's promotional efforts and competing duopolistic retailers' trade credits. International Journal of Production Economics, 204, 108-122.

Hou, H., Xie, J., Zhao, D., Chen, W., Li, Z., Ma, J., ... \& Dong, Z. (2016). Electrical vehicle wireless charging technology based on Energy Internet application in China. Procedia Computer Science, 83, 1332-1337.

Howell, S. T. (2018). Joint ventures and technology adoption: A Chinese industrial policy that backfired. Research Policy, 47(8), 1448-1462.

Iacocca, K. M., \& Mahar, S. (2019). Cooperative partnerships and pricing in the pharmaceutical supply chain. International Journal of Production Research, 57(6), 1724-1740.

Imran, M., et al. (2018). "Medicine supply chain model for an integrated healthcare system with uncertain product complaints." Journal of Manufacturing Systems 46: 13-28.

Jabarzare, N., and Rasti-Barzoki, M. (2019). A game theoretic approach for pricing and determining quality level through coordination contracts in a dual-channel supply chain including manufacturer and packaging company. International Journal of Production Economics.

Jensen, J., Rust, E., \& Mackool, S. (2018). Inflation, Interest Rates, and Equipment Finance: Anticipating and Adapting to a Changing Economic Environment. The Journal of Equipment Lease Financing (Online), 36(3), 1-5.

Jing, B., \& Seidmann, A. (2014). Finance sourcing in a supply chain. Decision Support Systems, $58,15-20$. 
Johari, M., \& Hosseini-Motlagh, S. M. (2020). Coordination contract for a competitive pharmaceutical supply chain considering corporate social responsibility and pricing decisions. RAIRO-Operations Research, 54(5), 1515-1535.

Juneja, J. A., \& Amar, A. D. (2018). An organizational capital decision model for knowledgeintensive organizations. IEEE Transactions on Engineering Management, 65(3), 417-433.

Kall, P., et al. (1994). Stochastic programming, Springer.

Kanda, A. and S. Deshmukh (2008). "Supply chain coordination: perspectives, empirical studies and research directions." International journal of production economics 115(2): 316-335.

Kang, H. D. (2018). Pharmaceutical Start-ups' Technology and Financing Strategy. Journal of Pharmaceutical Innovation, 13(4), 301-312.

Katz, R., Rebentisch, E. S., and Alien, T. J. (1996). A study of technology transfers in a multinational cooperative joint venture. IEEE Transactions on Engineering Management, 43(1), 97-105.

Kaya, O., and Ghahroodi, S. R. (2018). Inventory control and pricing for perishable products under age and price dependent stochastic demand. Mathematical Methods of Operations Research, 88(1), 1-35.

Kelle, P., et al. (2012). "Pharmaceutical supply chain specifics and inventory solutions for a hospital case." Operations Research for Health Care 1(2-3): 54-63.

Khodabakhshi, A., Mashreghi, H., and Emami, S. (2019). Coordination of a Three-echelon Pharma-Supply Chain with Corporate Social Responsibility Under Spanning and Pairwise Revenue Sharing Contracts. Paper presented at the 2019 15th Iran International Industrial Engineering Conference (IIIEC).

Kim, D. (2005). An integrated supply chain management system: a case study in healthcare sector. International Conference on Electronic Commerce and Web Technologies, Springer.

Ko, N., Jeong, B., Seo, W., and Yoon, J. (2019). A transferability evaluation model for intellectual property. Computers and Industrial Engineering, 131, 344-355.

Kouvelis, P., Xiao, Y., and Yang, N. (2015). PBM competition in pharmaceutical supply chain: Formulary design and medicine pricing. Manufacturing and Service Operations Management, 17(4), 511-526.

Krichanchai, S., \& MacCarthy, B. L. (2017). The adoption of vendor managed inventory for hospital pharmaceutical supply. The International Journal of Logistics Management.

Lauton, F., Rothkopf, A., \& Pibernik, R. (2019). The value of entrant manufacturers: A study of competition and risk for donor-funded procurement of essential medicines. European Journal of Operational Research, 272(1), 292-312.

Lee, J., Hoetker, G., \& Qualls, W. (2015). Alliance experience and governance flexibility. Organization Science, 26(5), 1536-1551. 
Li, D., and Nagurney, A. (2015). A general multitiered supply chain network model of quality competition with suppliers. International Journal of Production Economics, 170, 336-356.

Li, K., Qiu, J., \& Wang, J. (2019). Technology conglomeration, strategic alliances, and corporate innovation. Management science, 65(11), 5065-5090.

Lieberman, M. B., and Montgomery, D. B. (1988). First-mover advantages. Strategic management journal, 9(S1), 41-58.

Liu, J., Mantin, B., and Wang, H. (2014). Supply chain coordination with customer returns and refund-dependent demand. International Journal of Production Economics, 148, 81-89.

Longstaff, F. A., and Schwartz, E. S. (2001). Valuing American options by simulation: a simple least-squares approach. The review of financial studies, 14(1), 113-147.

Marzband, M., Ardeshiri, R. R., Moafi, M., and Uppal, H. (2017). Distributed generation for economic benefit maximization through coalition formation-based game theory concept. International Transactions on Electrical Energy Systems, 27(6), e2313.

Masoumi, A. H., Yu, M., and Nagurney, A. (2012). A supply chain generalized network oligopoly model for pharmaceuticals under brand differentiation and perishability. Transportation Research Part E: Logistics and Transportation Review, 48(4), 762-780.

Mellewigt, T., and Das, T. K. (2010). Alliance structure choice in the telecommunications industry: between resource type and resource heterogeneity. International Journal of Strategic Change Management, 2(2-3), 128-144.

Moiseeva, E., Wogrin, S., and Hesamzadeh, M. R. (2017). Generation flexibility in ramp rates: Strategic behavior and lessons for electricity market design. European Journal of Operational Research, 261(2), 755-771.

Moon, I., Feng, X. H., \& Ryu, K. Y. (2015). Channel coordination for multi-stage supply chains with revenue-sharing contracts under budget constraints. International Journal of Production Research, 53(16), 4819-4836.

Moslemi, S., Sabegh, M. H. Z., Mirzazadeh, A., Ozturkoglu, Y., \& Maass, E. (2017). A multiobjective model for multi-production and multi-echelon closed-loop pharmaceutical supply chain considering quality concepts: NSGAII approach. International Journal of System Assurance Engineering and Management, 8(2), 1717-1733.

Moulin, H. (1987). Equal or proportional division of a surplus, and other methods. International Journal of Game Theory, 16(3), 161-186.

Müller, A., and Zaby, A. K. (2019). Research joint ventures and technological proximity. Research Policy, 48(5), 1187-1200.

Mulvey, J. M., et al. (1997). "Strategic financial risk management and operations research." European Journal of Operational Research 97(1): 1-16. 
Nash, J. F. (1950). Equilibrium points in n-person games. Proceedings of the national academy of sciences, 36(1), 48-49.

National Center for Health, S. (2016). Health, United States. In Health, United States, 2015: With Special Feature on Racial and Ethnic Health Disparities. Hyattsville (MD): National Center for Health Statistics (US).

Nematollahi, M., Hosseini-Motlagh, S.-M., and Heydari, J. (2017). Economic and social collaborative decision-making on visit interval and service level in a two-echelon pharmaceutical supply chain. Journal of cleaner production, 142, 3956-3969.

Nematollahi, M., Hosseini-Motlagh, S. M., Ignatius, J., Goh, M., \& Nia, M. S. (2018). Coordinating a socially responsible pharmaceutical supply chain under periodic review replenishment policies. Journal of Cleaner Production, 172, 2876-2891.

Nicholson, L., et al. (2004). "Outsourcing inventory management decisions in healthcare: Models and application." European Journal of Operational Research 154(1): 271-290.

Nichols, N. A. (1994). Scientific Management at Merck: An Interview with CFO Judy Lewent. Harvard business review, 72(1), 88-99.

Nocke, V., and Schutz, N. (2018). Multiproduct-Firm Oligopoly: An Aggregative Games Approach. Econometrica, 86(2), 523-557.

Olsson, A., Sandberg, G., \& Dahlblom, O. (2003). On Latin hypercube sampling for structural reliability analysis. Structural safety, 25(1), 47-68.

Ouenniche, J., Boukouras, A., and Rajabi, M. (2016). An Ordinal Game Theory Approach to the Analysis and Selection of Partners in Public-Private Partnership Projects. Journal of Optimization Theory and Applications, 169(1), 314-343.

Pape, U., and Schmidt-Tank, S. (2004). Valuing joint ventures using real options. ESCP-EAP working paper, (7).

Park, B. J. R., Srivastava, M. K., and Gnyawali, D. R. (2014). Walking the tight rope of coopetition: Impact of competition and cooperation intensities and balance on firm innovation performance. Industrial Marketing Management, 43(2), 210-221.

Parkhe, A. (1993). Strategic alliance structuring: A game theoretic and transaction cost examination of interfirm cooperation. Academy of Management Journal, 36(4), 794-829.

Parr, R. (2012). Royalty rates for licensing intellectual property. John Wiley and Sons.

Pereira, M. V., Granville, S., Fampa, M. H., Dix, R., \& Barroso, L. A. (2005). Strategic bidding under uncertainty: a binary expansion approach. IEEE Transactions on Power Systems, 20(1), 180-188.

Petrou, P. (2016). Long-term effect of tendering on prices of branded pharmaceutical products. Health Policy and Technology, 5(1), 40-46. 
Pindyck, R. S. (1993). Investments of uncertain cost. Journal of financial Economics, 34(1), 5376.

Pitkethly, R. (1997). The valuation of patents: a review of patent valuation methods with consideration of option-based methods and the potential for further research. Research Papers in Management Studies-University of Cambridge Judge Institute of Management Studies.

Poppo, L., and Zenger, T. (2002). Do formal contracts and relational governance function as substitutes or complements? Strategic Management Journal, 23(8), 707-725.

Prak, D., Teunter, R., \& Riezebos, J. (2015). Periodic review and continuous ordering. European Journal of Operational Research, 242(3), 820-827.

Puigjaner, L., \& Laínez, J. M. (2008). Capturing dynamics in integrated supply chain management. Computers \& Chemical Engineering, 32(11), 2582-2605.

Rastogi, M., \& Singh, S. R. (2019a). An inventory system for varying deteriorating pharmaceutical items with price-sensitive demand and variable holding cost under partial backlogging in healthcare industries. Sädhanā, 44(4), 1-10.

Rastogi, M., \& Singh, S. R. (2019b). A Pharmaceutical Inventory Model for Varying Deteriorating Items with Price Sensitive Demand and Partial Backlogging Under the Effect of Learning. International Journal of Applied and Computational Mathematics, 5(3), 1-18.

Rego, N., Claro, J., and de Sousa, J. P. (2014). A hybrid approach for integrated healthcare cooperative purchasing and supply chain configuration. Health care management science, 17(4), 303-320.

Reuer, J. J., and Arino, A. (2007). Strategic alliance contracts: Dimensions and determinants of contractual complexity. Strategic Management Journal, 28(3), 313-330.

Richards, T. J., \& Rickard, B. J. (2014). Patents as options: path-dependency and patent value. European Review of Agricultural Economics, 41(5), 817-841.

Rida, N. A., \& Ibrahim, M. I. M. (2018). Medicines pricing policy and strategies in developing countries: A review. Social and administrative aspects of pharmacy in low-and middleincome countries, 111-128.

Rida, N. A., Ibrahim, M. M., \& Babar, Z. U. D. (2019). Relationship between pharmaceutical pricing strategies with price, availability, and affordability of cardiovascular disease medicines: surveys in Qatar and Lebanon. BMC health services research, 19(1), 1-14.

Sahebi, H., Nickel, S., and Ashayeri, J. (2015). Joint venture formation and partner selection in upstream crude oil section: goal programming application. International Journal of Production Research, 53(10), 3047-3061.

San Cristóbal, J. R. (2012). Game Theory and the Shapley Value Applied to a Vessel's Drydocking. Journal of Ship Production and Design, 28(4), 160-163. 
Sarathi, G. P., et al. (2014). "An integrated revenue sharing and quantity discounts contract for coordinating a supply chain dealing with short life-cycle products." Applied Mathematical Modelling 38(15-16): 4120-4136.

Sarmah, A., De Giovanni, D., and De Giovanni, P. (2019). Compulsory licenses in the pharmaceutical industry: Pricing and RandD strategies. European Journal of Operational Research.

Schaeffer, R. (2009). "Closing the medication safety loop." Health management technology 30(3): 30-32.

Schneeweiss, S. (2007). Reference medicine programs: effectiveness and policy implications. Health policy, 81(1), 17-28.

Schwartz, E. S. (2004). "Patents and RandD as real options." Economic Notes 33(1): 23-54.

Schwartz, E. S., and Moon, M. A. (1996). Evaluating research and development investments. John E. Anderson Graduate School of Management at UCLA.

Sercu, P. (2009). International finance: Theory into practice. Princeton University Press.

Sazvar, Z., Zokaee, M., Tavakkoli-Moghaddam, R., Salari, S. A. S., \& Nayeri, S. (2021). Designing a sustainable closed-loop pharmaceutical supply chain in a competitive market considering demand uncertainty, manufacturer's brand and waste management. Annals of Operations Research, 1-32.

Shafiei Khah, M., \& Amiri, A. (2014). Petroleum Contracts in Iran. European Online Journal of Natural and Social Sciences: Proceedings, 3(3 (s)), pp-375.

Shah, N. (2004). "Pharmaceutical supply chains: key issues and strategies for optimization." Computers and chemical engineering 28(6-7): 929-941.

Sinha, U. B. (2001). International joint venture, licensing and buy-out under asymmetric information. Journal of Development Economics, 66(1), 127-151.

Skovsgaard, L., and Jensen, I. G. (2018). Recent trends in biogas value chains explained using cooperative game theory. Energy Economics, 74, 503-522.

Stecca, G., et al. (2016). "Design and operation of strategic inventory control system for medicine delivery in healthcare industry." IFAC-PapersOnLine 49(12): 904-909.

Sterman, J. D. (2000). Business dynamics: systems thinking and modeling for a complex world: Jeffrey J. Shelstad, Indianapolis, IN.

Sullivan, P. H. (2000). Value driven intellectual capital: how to convert intangible corporate assets into market value. John Wiley and Sons, Inc.

Sun, P., Qu, Z., \& Liao, Z. (2018). How and when do subnational institutions matter for R\&D investment? Evidence from the Chinese pharmaceutical sector. IEEE Transactions on Engineering Management, 65(3), 379-391. 
Sun, Y., Chen, X., and Luo, Y. (2019, April). Profit Allocation Method in Influenza Vaccine Supply Chain by Risk Compensation. In 2019 IEEE 6th International Conference on Industrial Engineering and Applications (ICIEA) (pp. 511-515). IEEE.

Taleizadeh, A. A., Haji-Sami, E., and Noori-daryan, M. (2019). A robust optimization model for coordinating pharmaceutical reverse supply chains under return strategies. Annals of Operations Research, 1-22.

Taleizadeh, A. A., Zarei, H. R., \& Sarker, B. R. (2017). An optimal control of inventory under probabilistic replenishment intervals and known price increase. European Journal of Operational Research, 257(3), 777-791.

Timmermans, K., \& Hutadjulu, T. (2000). The TRIPS Agreement and Pharmaceuticals, Report of an ASEAN Workshop on the TRIPs Agreement and its Impact on Pharmaceuticals. Jakarta, Indonesia, 91.

Tsay, A. A. (1999). "The quantity flexibility contract and supplier-customer incentives." Management Science 45(10): 1339-1358.

Tsui, M., Wilson, D. I., Merry, H., Phulwani, K., \& Dooley, M. J. (2008). Implementing a Hospital Vendor-Managed Inventory System. Journal of pharmacy practice and research, 38(1), 40-43.

Uthayakumar, R. and S. Priyan (2013). "Pharmaceutical supply chain and inventory management strategies: Optimization for a pharmaceutical company and a hospital." Operations Research for Health Care 2(3): 52-64.

Vahdat, V., \& Vahdatzad, M. A. (2017). Accelerated Benders' Decomposition for Integrated Forward/Reverse Logistics Network Design under Uncertainty. Logistics, 1(2), 11.

Ventosa, M., Baillo, A., Ramos, A., \& Rivier, M. (2005). Electricity market modeling trends. Energy policy, 33(7), 897-913.

Vishwasrao, S. (2007). Royalties vs. fees: How do firms pay for foreign technology?. International Journal of Industrial Organization, 25(4), 741-759.

Vogler, S., and Martikainen, J. E. (2015). Pharmaceutical pricing in Europe. In Pharmaceutical prices in the 21st century (pp. 343-370): Springer.

Wang, K. J., Lin, Y. S., and Jonas, C. P. (2011). Optimizing inventory policy for products with time-sensitive deteriorating rates in a multi-echelon supply chain. International Journal of Production Economics, 130(1), 66-76.

Weraikat, D., Zanjani, M. K., and Lehoux, N. (2016). Two-echelon pharmaceutical reverse supply chain coordination with customers' incentives. International Journal of Production Economics, 176, 41-52.

World Health Organization. (2002). Implications of the Doha Declaration on the TRIPS Agreement and public health (No. WHO/EDM/PAR/2002.3). World Health Organization. 
World Health Organization. (2010). World health statistics 2010. World Health Organization.

Wright, D. J. (2004). The medicine bargaining game: pharmaceutical regulation in Australia. Journal of health economics, 23(4), 785-813.

Wu, D., \& Ierapetritou, M. (2007). Hierarchical approach for production planning and scheduling under uncertainty. Chemical Engineering and Processing: Process Intensification, 46(11), 1129-1140.

Wu, Q., Ren, H., Gao, W., Ren, J., and Lao, C. (2017). Profit allocation analysis among the distributed energy network participants based on Game-theory. Energy, 118, 783-794.

Xiong, H., Chen, B., and Xie, J. (2011). A composite contract based on buy back and quantity flexibility contracts. European Journal of Operational Research, 210(3), 559-567.

Yadav, D., Kumari, R., Kumar, N., \& Sarkar, B. (2021). Reduction of waste and carbon emission through the selection of items with cross-price elasticity of demand to form a sustainable supply chain with preservation technology. Journal of Cleaner Production, 297, 126298.

Yan, M. R. (2011). A fuzzy logic enhanced bargaining model for business pricing decision support in joint venture projects. Journal of Business Economics and Management, 12(2), 234-247.

Yan, M. R., and Hsueh, S. L. (2011). Contribution-Based Profit-Sharing Scheme for Joint Ventures. Technological and Economic Development of Economy, (3), 445-458.

Yan, M. and T. Yang (2012). "Dynamic gaming and risk-incorporated bargaining decision support model for strategic alliance projects." APPLIED MATHEMATICS \& INFORMATION SCIENCES 6(2).

Yoon, J., Rosales, C., \& Talluri, S. (2018). Inter-firm partnerships-strategic alliances in the pharmaceutical industry. International Journal of Production Research, 56(1-2), 862-881.

Zaharoff, H. G. (2004). Setting values and royalty rates for medical and life science businesses.

Journal of Biolaw and Business, 7, 07-09.

Zahiri, B., et al. (2018). "Design of a pharmaceutical supply chain network under uncertainty considering perishability and substitutability of products." Information Sciences 423: 257283.

Zhang, S. 2007. "Risk Sharing in Joint Venture Projects." PhD thesis, Kyoto University.

Zhang, T., Y. Qu and G. He (2019). "Pricing strategy for green products based on disparities in energy consumption." IEEE Transactions on Engineering Management. doi: 10.1109/TEM.2019.2907872.

Zhao, H., Xiong, C., Gavirneni, S., and Fein, A. (2012). Fee-for-service contracts in pharmaceutical distribution supply chains: design, analysis, and management. Manufacturing and Service Operations Management, 14(4), 685-699. 


\section{Appendixes}

\subsection{Proof of Proposition (3.1)}

Optimizing each side of Eq.3.15 as a maximization problem is frustrating and even by using Lagrangian multiplication, the closed form of solutions needs an interpretation of parametric values which is cumbersome. But it should be noted that the aim of $r, L$ and $\varphi$ are to distribute the cooperative synergy in a fair manner and this synergy is maximized, when $(a-b . p) p-v(a-b . p)-F$ or the total profit of $J V$ is maximized. This is achieved by choosing

appropriate $p$, i.e. $p=\frac{a+v \cdot b}{2 b}$. To clarify this, note that $L$ can be determined as a function of other variables and parameters by rewriting Eq. 15. Doing so, $L$ will be as Eq. A3.1:

$$
L=\frac{A-B}{C}
$$

Where:

$$
\begin{aligned}
& B=\varphi \cdot\left(1-\tau_{F F, 2}\right) \cdot(p(a-b \cdot p)-F-v(a-b \cdot p)-r \cdot p \cdot(a-b \cdot p))
\end{aligned}
$$

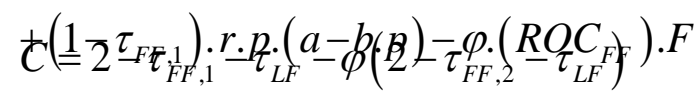

In Eq. A3.1, the nominator represents the gain function of $L F$ without $L$ minus the gain function of $F F$ without $L$. Based on the assumption that $L \geq 0$ and the profit function of the partners are equally based on the proportional contract, the gain function of $L F$ without $L$ is equal or greater than the gain function of $F F$ without $L$. So, the nominator of Eq. A3.1 is positive and the denominator of Eq. A3.1 should be positive to make $L \geq 0$. This imposes the constraint $\tau_{F F, 1}+\tau_{L F}-2-\varphi\left(\tau_{F F, 2}+\tau_{L F}-2\right) \geq 0$ or equivalently $\varphi \leq \frac{2-\tau_{F F, 1}-\tau_{L F}}{2-\tau_{F F, 2}-\tau_{L F}}$ in the model constraints.

Replacing Eq. A3.1 into the left-hand side of Eq.3.15, the gain function of $F F$ is rewritten as the following: 


$$
\begin{aligned}
& \pi_{J V, F F}-\pi_{S P, F F}= \\
& (\varphi-1)\left(\frac{\left(1-\tau_{F F, 1}\right)\left(1-\tau_{L F}\right)(p-v)(a-b \cdot p)}{\tau_{F F, 1}+\tau_{L F}-2-\varphi\left(\tau_{F F, 2}+\tau_{L F}-2\right)}+\right. \\
& \left.\frac{F \cdot R O C_{L F} .\left(1-\tau_{F F, 1}\right)-F \cdot \tau_{L F} \cdot\left(1-\tau_{F F, 1}\right)+F \cdot \varphi \cdot\left(R O C_{F F} \cdot\left(1-\tau_{L F}\right)-R O C_{L F} \cdot\left(1-\tau_{F F, 2}\right)\right)}{\tau_{F F, 1}+\tau_{L F}-2-\varphi\left(\tau_{F F, 2}+\tau_{L F}-2\right)}\right)
\end{aligned}
$$

Differentiating Eq. A3.2 with respect to $p$ and setting it equal to zero, the value of $p$ that maximizes the gain function of the partners will be $p=\frac{a+v \cdot b}{2 b}$. Also based on the above discussion, the second derivative of the partner's gain with respect to $p$ is negative which conforms the concavity $\pi_{J V, F F}-\pi_{S P, F F}$ relative to $p$ and the global optimality of $p=\frac{a+v \cdot b}{2 b}$.

\subsection{Proof of Lemma (3.1)}

To prove the lemma, some remarks should be noted. First, in real-world situations, $O_{L F}$ is usually positive. This is because the exclusive markets of new technologies could provide extra benefits for the first mover of the market (Lieberman and Montgomery, 1988). Second, it is possible for $F F$, which is the owner of the rare technology, to find more profitable markets in other countries. $F F$ could even invest in his R\&D projects instead of the local market, and such projects might be predicted to be more beneficial. Third, when $O_{L F}$ is negative, then based on Eq. 3.20 there is no positive gain for $L F$ and this indicates the formation of $J V$ is not affordable. Nevertheless, when $O_{L F}$ is positive, we could obtain a feasible solution for $J V$ by setting $\varphi=0$ which results in a positive value for $L P$ as another variable of the model $A$ 3.2. Therefore, the lemma is proved.

\subsection{Proof of the negativity of radicand in Eq. 3.23 when $\left(\boldsymbol{O}_{F F} \cdot\left(1-\tau_{L F}\right)-\boldsymbol{O}_{L F} \cdot\left(1-\tau_{F F .2}\right)\right)>0$}


The following inequalities prove this. (Note that in the proofs, the assumptions $0 \leq \tau_{L F}, \tau_{F F, 1}, \tau_{F F, 2}<1$ and $\tau_{F F, 1} \geq \tau_{F F, 2}$ is valid).

$$
\begin{aligned}
& \left\{\begin{array}{c}
O_{L F} \cdot\left(\tau_{F F, 1}-\tau_{F F, 2}\right)<O_{L F} \cdot\left(1-\tau_{F F, 2}\right) \\
O_{F F} \cdot\left(2-\tau_{L F}-\tau_{F F, 1}\right)>O_{F F} \cdot\left(1-\tau_{L F}\right) \rightarrow-O_{F F} \cdot\left(2-\tau_{L F}-\tau_{F F, 1}\right)<-O_{F F} \cdot\left(1-\tau_{L F}\right)
\end{array}\right\} \rightarrow \\
& O_{L F} \cdot\left(\tau_{F F, 1}-\tau_{F F, 2}\right)-O_{F F} \cdot\left(2-\tau_{L F}-\tau_{F F, 1}\right)<O_{L F} \cdot\left(1-\tau_{F F, 2}\right)-O_{F F} \cdot\left(1-\tau_{L F}\right)<0
\end{aligned}
$$

So, the term $H$ is negative, and we know that the term $G$ is positive by assumption. Therefore, the multiplication of the two terms is negative, which makes the radicand negative as well.

\subsection{Proof of the optimality of $\varphi^{*}$ in Eq. 25 when $\left(o_{F F} \cdot\left(1-\tau_{L F}\right)-O_{L F} \cdot\left(1-\tau_{F F, 2}\right)\right)=0$}

In this case, the coefficient $A$ and $B$ of $Z^{\varphi}(\varphi)$ in Eq. 3.22 is zero and therefore, the positivity or the negativity of $Z^{\varphi}(\varphi)$ is specified by the sign of the coefficient $C$. Applying $O_{F F} \cdot\left(1-\tau_{L F}\right)=O_{L F} \cdot\left(1-\tau_{F F, 2}\right)$ and doing some manipulation, the coefficient $C$ in Eq. 3.22 will be

equal to $\left(\tau_{F F, 1}-\tau_{F F, 2}\right) \cdot\left(1-\tau_{F F, 2}\right) . O_{L F}$ which is always non-negative. The non-negativity of $C$ indicates that the optimal value of $\varphi$ is the maximum possible value of this variable i.e., $\varphi^{*}$ according to Eq. 3.25.

\subsection{Proof of the optimality of $\varphi^{*}=0$ in the state where $\left(o_{F F} .\left(1-\tau_{L F}\right)-O_{L F} \cdot\left(1-\tau_{F F, 2}\right)\right)<0$} and $O_{L F} \cdot\left(\tau_{F F, 1}-\tau_{F F, 2}\right)-O_{F F} \cdot\left(2-\tau_{L F}-\tau_{F F, 1}\right) \leq 0$

Clearly, in this state, there are two real roots for $\varphi$ which make $Z^{\varphi}=0$ in Eq. 3.22. One of them is less than 1, and the other is greater than 1 . If $\varphi_{1}$ indicates the root which is less than 1 , then 
when $0 \leq \varphi \leq \varphi_{1}, Z^{\varphi}$ is positive and when $\varphi_{1} \leq \varphi \leq 1, Z^{\varphi}$ is negative. In the other hand, regarding the non-negativity of $L P$ and denoting the value of $\varphi$ which make $L P=0$ by $\varphi_{3}$, we should have $\varphi \leq \varphi_{3}$. Therefore, two conditions might occur. If $\varphi_{3} \leq \varphi_{1}$ then $\varphi_{3}$ is never the optimal solution because $Z(\varphi)$ is a descending function from $\varphi=0$ up to $\varphi_{3}$. But if $\varphi_{3}>\varphi_{1}$, then, $Z(\varphi)$ is an ascending function from $\varphi_{1}$ up to $\varphi_{3}$ and we need to compare $Z\left(\varphi_{3}\right)$ with $Z(0)$ to find the optimal solution among them. Taking into account model A3.2, $Z(0)$ and $Z\left(\varphi_{3}\right)$ are shown in Eq. A3.4 and A3.5, respectively.

$$
\begin{aligned}
& Z(0)=\frac{O_{L F} \cdot\left(1-\tau_{F F, 1}\right)}{2-\tau_{L F}-\tau_{F F, 1}} \\
& Z\left(\varphi_{3}\right)=\frac{O_{L F} \cdot O_{F F}}{O_{L F}+O_{F F}}
\end{aligned}
$$

Nonetheless, $Z(0)$ is always greater than $Z\left(\varphi_{3}\right)$. To prove this, we show that $Z\left(\varphi_{3}\right) \geq Z(0)$ leads to a contradiction. This is due to the fact that if $Z\left(\varphi_{3}\right) \geq Z(0)$, the following inequality will be true:

$$
\begin{aligned}
& \frac{O_{L F} \cdot O_{F F}}{O_{L F}+O_{F F}} \geq \frac{O_{L F} \cdot\left(1-\tau_{F F, 1}\right)}{2-\tau_{L F}-\tau_{F F, 1}} \rightarrow\left(2-\tau_{L F}-\tau_{F F, 1}\right) \cdot O_{L F} \geq\left(1-\tau_{F F, 1}\right) \\
& \left(O_{L F}+O_{F F}\right) \rightarrow\left(1-\tau_{L F}\right) \cdot O_{L F}+\left(1-\tau_{F F, 1}\right) \cdot O_{L F}-\left(1-\tau_{F F, 1}\right) \cdot O_{L F}-\left(1-\tau_{F F, 1}\right) \cdot O_{F F} \geq 0 \\
& \rightarrow\left(1-\tau_{L F}\right) \cdot O_{L F}-\left(1-\tau_{F F, 1}\right) \cdot O_{F F} \geq 0
\end{aligned}
$$

The last inequality in A3.6 is in contrast with the base assumptions of Situation (3.3), and this shows that $Z\left(\varphi_{3}\right)$ could not be greater than $Z(0)$. So, $\varphi^{*}=0$ is the optimal solution of this state.

\section{6: Proof of Proposition (7):}


When $R O C_{F F}$ is high enough to make $M G_{S}$ positive and confirm Licensee ownership as the optimal structure of ownership, then there is no share for $F F$, and the license income is determined by Eq.24. Substituting $\varphi=0$ and Eq. 24 into Eq. 20 and adding the stand-alone profit of $L F$ by it, the profit of $L F$ in Licensee ownership structure $\left(\pi_{J V, L F}^{L O S}\right)$ is determined by Eq. A3.7.

$$
\pi_{J V, L F}^{L O S}=\frac{1-\tau_{F F, 1}}{2-\tau_{L F}-\tau_{F F, 1}} \cdot O_{L F}+R O C_{L F} . F
$$

On the other hand, we know from Proposition 3.6 that the shared ownership structure is optimal when $M G_{S}$ is positive. Regarding $M G_{S}$ definition as Eq. 3.26 and the positivity of $M G_{S}$, the following inequality is valid in shared ownership Structure:

$$
\frac{O_{F F}}{\left(1-\tau_{F F, 2}\right)} \geq \frac{O_{L F}}{\left(1-\tau_{L F}\right)} \rightarrow \frac{O_{L F}}{O_{L F}+O_{F F}} \leq \frac{1-\tau_{L F}}{2-\tau_{F F, 2}-\tau_{L F}}<\frac{1-\tau_{L F}}{2-\tau_{F F, 1}-\tau_{L F}}
$$

Moreover, regarding $L P^{*}=0, \varphi^{*}$ as Eq. 3.25 and adding the stand-alone profit of $L F$ by Eq. 3.20, the profit of $L F$ in shared ownership structure $\left(\pi_{J V, L F}^{S O S}\right)$ could be expressed by Eq. A3.9:

$$
\pi_{J V, L F}^{S O S}=\frac{O_{L F}}{O_{L F}+O_{F F}} \cdot O_{L F}+\frac{O_{L F}}{O_{L F}+O_{F F}} \cdot\left(R O C_{L F}\right) \cdot F
$$

Now, considering Eq. A3.7, A3.8, and A3.9, it is straightforwardly confirmed that $\pi_{J V, L F}^{L O S}>\pi_{J V, L F}^{S O S}$ and the proof is completed.

\section{1: Proof of Proposition 4.1:}

To prove this theorem, we consider the Lagrangian function of firm $i$ 's income in Eq. A3.10:

$$
L_{i}=p \cdot q_{i}-\left(C_{i}\left(q_{i}\right)+t c r^{i} \cdot q_{i}\right)-\lambda_{i}\left(q_{i}-x_{i}\right)+\mu_{i} \cdot q_{i} \quad \forall i
$$

Regarding the Lagrangian function, the first-order and second-order conditions for the equilibrium price will be as Eqs. A3.11 and A3.12 respectively. 


$$
\begin{aligned}
& \frac{\partial L_{i}}{\partial q_{i}}=0 \rightarrow \frac{\partial p}{\partial q_{i}} \cdot q_{i}-\frac{\partial C_{i}^{s}\left(q_{i}\right)}{\partial q_{i}}-t c r^{i}-\lambda_{i}+\mu_{i}=0 \stackrel{\frac{\partial p}{\partial q_{i}}=-\theta_{i}}{\Rightarrow} \\
& -\theta_{i} \cdot q_{i}-\frac{\partial C_{i}^{s}\left(q_{i}\right)}{\partial q_{i}}-t c r^{i}-\lambda_{i}+\mu_{i}=0 \quad \forall i \\
& \mu_{i} \cdot q_{i}=0 \quad \forall i \quad \forall i \\
& \lambda_{i} \cdot\left(q_{i}-P C_{i}\right)=0 \quad \forall i \\
& 0 \leq q_{i} \leq P C_{i} \quad \forall i \\
& \lambda_{i}, \mu_{i} \geq 0 \quad \forall i \\
& \frac{\partial^{2} L_{i}}{\partial\left(q_{i}\right)^{2}} \leq 0 \rightarrow-\theta_{i}-\frac{\partial^{2} C_{i}^{s}\left(q_{i}\right)}{\partial\left(q_{i}\right)^{2}} \leq 0
\end{aligned}
$$

Now, by reduction in the transaction cost of firm $i$, two conditions might happen:

i. If, in the equilibrium price before reducing transaction costs, the firm $i$ produces at its full capacity, then $q_{i}=x_{i}, \mu_{i}=0$ and $\lambda_{i} \geq 0$. Now, if transaction costs are decreased from $\operatorname{trc} c^{i}$ to $\operatorname{trc} c^{i}-\varepsilon$, then by changing $\lambda_{i}$ to $\lambda_{i}+\varepsilon$, the same equilibrium price will be resulted and therefore the equilibrium price will not change in this case.

ii. On the other hand, if in the equilibrium price, firm $i$ produces less than the maximum level of capacity, then $q_{i}<x_{i}, \lambda_{i}=0$ and $\mu_{i} \geq 0$. Now, as long as the production quantity is less than the capacity of the firm $i$, reducing in the transaction cost led to increases in the production quantity. To show this, Eq. A3.13 is considered:

$$
-\theta_{i} \cdot \frac{\partial q_{i}}{\partial t r c_{i}}-\frac{\partial^{2} C_{i}^{s}\left(q_{i}\right)}{\partial\left(q_{i}\right)^{2}} \cdot \frac{\partial q_{i}}{\partial t r c_{i}}-1=0 \stackrel{\frac{\partial p}{\partial q_{i}}=-\theta_{i}}{\rightarrow} \frac{\partial q_{i}}{\partial t r c_{i}}=\frac{1}{-\theta_{i}-\frac{\partial^{2} C_{i}^{s}\left(q_{i}\right)}{\partial\left(q_{i}\right)^{2}}}
$$

In Eq. A3.13, the denominator is negative and this proves the increase in the firm i's supply after reducing the transaction cost. On the other hand, regarding the positivity of the conjecture price in the equation $\theta_{i}=-\frac{\partial p}{\partial q_{i}}$, the decrease in the market equilibrium price and consequently, the increase in the market demand based on the equation $d=E-\alpha . p$ is proved. These explanations suggest that a lower transaction cost of a firm leads to more profit for that firm and the customers will not pay more than they would do prior to lowering the transaction cost. 